\title{
Visuell-räumliche Navigationsleistungen und parietales Cortexvolumen bei schizophrenen Patienten im Paradigma der „Virtuellen Realität““
}

\author{
Dissertation \\ zur Erlangung des Doktorgrades \\ der Mathematisch-Naturwissenschaftlichen Fakultäten \\ der Georg-August-Universität zu Göttingen
}

vorgelegt von:

Dipl.-Psych. Mirjana Ruhleder aus Bad Harzburg

Göttingen, 2006 
D7

Referent: Professor Doktor Eva Irle

Koreferent: Professor Doktor Marcus Hasselhorn

Tag der mündlichen Prüfung: 17.01.2007 


\section{Inhaltsverzeichnis}

0. Zusammenfassung

1. Theoretischer Hintergrund

1.1. Allgemeiner Überblick

1.1.1. Die Phänomenologie der Schizophrenie: Eine Einführung

1.1.2. Die Psychopathologie der Schizophrenie

1.1.3. Die Neuropsychologie der Schizophrenie

1.1.4. Die Neurobiologie der Schizophrenie

1.1.4.1. Der Frontallappen

1.1.4.2. Der Temporallappen

1.1.4.3. Der Parietallappen 10

1.1.4.3.1. Neuroanatomie: Parietale Anomalien ……....................................

1.1.4.3.1.1. Empirische Befunde

1.1.4.3.1.2. Pearlsons Theorie heteromodaler Assoziationscortices ........... 12

1.1.4.3.2. Psychopathologie: Eine parietale Genese

1.1.4.3.3. Neuropsychologie: Defizite in ,parietalen“ Funktionen

1.2. Die aktuelle Forschungsmotivation 15

1.2.1. Das Konzept der „,visuell-räumlichen Navigation“

1.2.1.1. Ego- und allozentrische Repräsentationen $\quad 16$

1.2.1.2. Neuronale Korrelate der ego- und allozentrischen Repräsentationen _ $\quad 17$

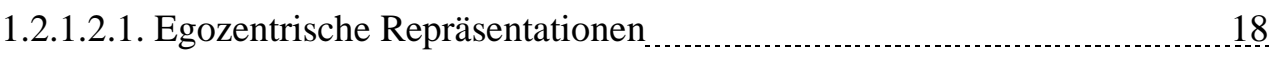

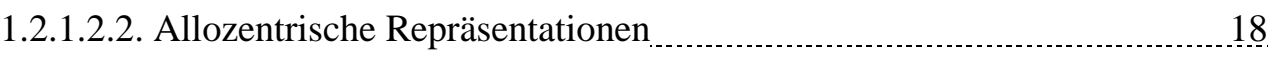

1.2.1.2.3. Das parieto-hippocampale Netzwerk

1.2.2. Objektivierung der visuell-räumlichen Navigation: Die Virtuelle Realität__........................ 20

1.2.2.1. Konventionelle neuropsychologische Testverfahren $\quad 20$

1.2.2.2. Das Paradigma der „Virtuellen Realität“

1.2.2.2.1. Begriffsdefinition: Was ist die „Virtuelle Realität“? .......................

1.2.2.2.2. Validitätsvorteile des VR-Paradigmas _ 23

1.2.2.2.3. Visuell-räumliche Navigationsprozesse 25

1.2.2.2.4. Das VR-Paradigma und schizophrene Testpersonen _............................

1.3. Zusammenfassung 27

2. Ableitung der Forschungshypothesen $\quad 28$

2.1. Zentrale Forschungshypothesen $\ldots$

2.1.1. Hypothesen Neuropsychologie: Visuell-räumliche Navigationsleistung _ _ $\quad 28$

2.1.2. Hypothesen Hirnvolumetrie: Parietale Cortexvolumina 28

2.1.3. Zusammenhangshypothesen Neuropsychologie - Hirnvolumetrie 28 
2.2.1. Hypothesen zu hippocampalen Arealen 29

2.2.2. Hypothesen zu schizophrenen Subgruppen 29

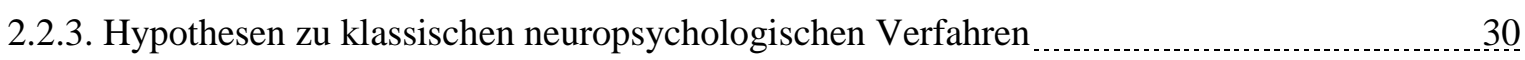

3. Methoden

3.1. Stichprobe

3.1.1. Stichprobenumfang 31

3.1.2. Rekrutierung der Versuchspersonen 31

3.1.3. Charakterisierung der Stichprobe 31

3.1.3.1. Ein- und Ausschlusskriterien

3.1.3.2. Vergleich der Probandengruppen - Soziodemographische Daten _ $\quad 32$

3.1.3.3. Charakterisierung der schizophrenen Probandengruppe 34

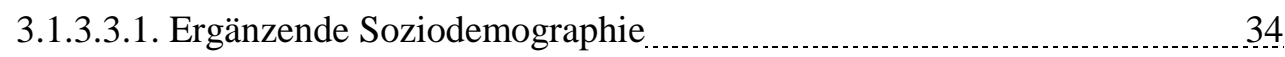

3.1.3.3.2. Klinische Erhebungsverfahren

3.1.3.3.2.1. SKID

3.1.3.3.2.2. GAF

3.1.3.3.2.3. CGI 35

3.1.3.3.2.4. BPRS 35

3.1.3.3.2.5. SAPS und SANS $\ldots$

3.1.3.3.3. Psychopathologische Kennwerte $\quad 37$

3.1.3.3.4. Schizophrene Subgruppen

3.2. Variation der unabhängigen Variablen $\quad 40$

3.2.1. Überblick 40

3.2.2. Die „Virtuellen Realitäten“

3.2.2.1. Methodische Realisierung

3.2.2.1.1. Das ,virtuelle Labyrinth“

3.2.2.1.2. Der ,virtuelle Park“

3.2.2.1.3. Anzahl, Dauer und Präsentationsform der Testdurchläufe _................... 43

3.2.2.2. Technische Realisierung

3.2.2.2.1. Ausgabesysteme: Das Head Mounted Display ..................................... 45

3.2.2.2.2. Eingabesysteme: Die Computer- und Versuchssteuerungssysteme.......... 46

3.3. Operationalisierung der abhängigen Variablen 46

3.3.1. Überblick 46

3.3.2. Erfassung der ego- und allozentrischen Navigationsleistung $\ldots \ldots \ldots$ 
3.3.3. Erfassung der hirnanatomischen Variablen

3.3.3.1. Parameter und Aufnahmetechnik zur Erhebung der MRT-Scans _ 48

3.3.3.2. Auswertungsprozedere 49

3.3.3.2.1. Software „Curry“

3.3.3.2.2. Hirnanatomische Grenzlinien/Manuelle Segmentierung _...................... 49

3.3.4. Neuropsychologische Testverfahren

3.3.4.1. HAWIE-R

3.3.4.2. WMS-R

3.3.4.3. TMT-A und TMT-B

3.4. Versuchsaufbau und -ablauf

3.4.1. Aufklärung und Einverständniserklärung _ 54

3.4.2. Versuchsteil 1: Fragebögen, neuropsychologische (und psychopathologische) Testung 55

3.4.3. Versuchsteil 2: Die Virtuelle Realität

3.4.3.1. Instruktionen

3.4.3.2. Trainingstrials

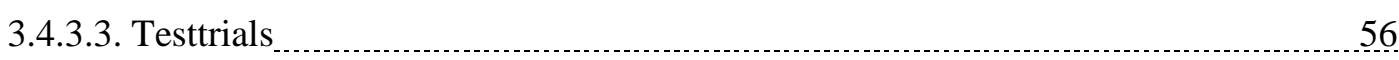

3.4.4. Versuchsteil 3: Magnetresonanztomographie 57

3.4.4.1. Aufklärung und Einverständniserklärung $\quad 57$

3.4.4.2. Erhebung der hirnanatomischen Daten $\quad 58$

3.4.5. Zusammenfassung 58

3.5. Datenanalyse $\quad 5$

4. Ergebnisse

4.1. Zentrale Forschungshypothesen $\quad 60$

4.1.1. Neuropsychologie: Visuell-räumliche Navigationsleistung $\quad 60$

4.1.2. Hirnvolumetrie: Parietale Cortexvolumina 64

4.1.3. Zusammenhänge Neuropsychologie - Hirnvolumetrie $\quad 65$

4.1.3.1. Kontrollgruppe 66

4.1.3.2. Schizophrene Probandengruppe $\ldots$

4.2. Explorative und validierende Hypothesen

4.2.1. Hippocampale Areale $\ldots \ldots \ldots$

4.2.2. Schizophrene Subgruppen

4.2.2.1. Visuell-räumliche Navigationsleistung $\ldots 9$

4.2.2.2. Parietale Cortexvolumina 72

4.2.3. Klassische neuropsychologische Verfahren 74 
5.1. Zentrale Forschungshypothesen 76

5.1.1. Visuell-räumliche Navigationsleistung (Hypothesen 1 und 2) 76

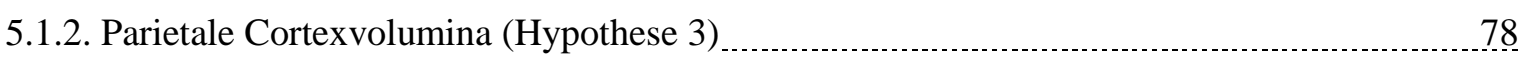

5.1.3. Zusammenhänge Neuropsychologie - Hirnvolumetrie (Hypothesen 4 und 5) _..................... 79

5.1.4. Zusammenfassung zentrale Forschungshypothesen

5.2. Explorative und validierende Hypothesen 81

5.2.1. Hippocampale Areale (Hypothesen 6 und 7) 81

5.2.2. Schizophrene Subgruppen (Hypothesen 8 und 9) 82

5.2.3. Klassische neuropsychologische Verfahren (Hypothesen 10 und 11) _.............................. 83

5.3. Zusammenfassung $\ldots$

5.4. Abschließende Bemerkungen

6. Literaturverzeichnis 92

7. Anhang 116

8. Wissenschaftlicher Bildungsgang der Verfasserin 151 


\section{Zusammenfassung}

Erst wenige Arbeiten haben sich mit parietalen Dysfunktionen im Rahmen der Schizophrenie beschäftigt. Daher zielt die vorliegende Studie darauf ab, Defizite in klassischen „parietalen“ Hirnfunktionen mit Hilfe einer virtuellen Navigationsaufgabe zu erfassen sowie eine diesbezügliche Involvierung parietaler Cortexareale zu bestätigen.

$\mathrm{Zu}$ diesem Zweck durchliefen schizophrene Patienten sowie Kontrollpersonen mit dem „virtuellen“ Labyrinth“ und dem „virtuellen Park“ zwei unterschiedliche dreidimensionale Testumgebungen, innerhalb derer sie einen Zielpunkt finden und eine vorgegebene Route memorieren mussten. So wurden die allozentrische und die egozentrische Navigationsleistung der Probanden erhoben. Um diese neuropsychologischen Variablen mit neuronanatomischen Variablen - dem Parietallappen - assoziieren zu können, wurden zudem die Cortices der Probanden mit Hilfe eines Magnetresonanztomographen erfasst. Explorativ wurde zum einen die Beteiligung hippocampaler Areale untersucht; zum anderen wurde innerhalb einer Subgruppenanalyse geprüft, ob sich differentielle neuropsychologische oder hirnantomische Profile bei den verschiedenen schizophrenen Subtypen beobachten ließen. Zudem kamen zur Erzielung einer besseren Vergleichbarkeit zwei klassische neuropsychologische Testinstrumente zum Einsatz, in denen schizophrene Patienten typischerweise Defizite aufweisen: Die Wechsler Memory Scale-Revised sowie der Trail Making Test A und B.

Die zentralen Versuchshypothesen konnten anhand der empirischen Daten weitgehend bestätigt werden: Schizophrene Patienten wiesen sowohl im ego-, als auch im allozentrischen Raum eine defizitäre Navigationsleistung auf, die der von gesunden Kontrollpersonen unterlegen war. Darüber hinaus ließen sich in der Patientengruppe Volumenreduktionen im globalen und superioren Parietalcortex nachweisen. Die (reduzierten) parietalen Cortexvolumina der schizophrenen Probandengruppe standen des Weiteren in einem funktionalen Zusammenhang zu den beobachteten Navigationsdefiziten: Je kleiner die parietalen Cortexvolumina waren, desto schlechter waren auch die Navigationsleistungen im ego- und allozentrischen Raum.

Innerhalb der explorativen Analyse zeigte sich bei den schizophrenen Probanden eine deutliche bilaterale Volumenreduktion der Hippocampi. Zusammenhänge zu (defizitären) ego- oder allozentrischen Navigationsleistungen ließen sich allerdings nicht feststellen.

Die Subtypenanalyse wies des Weiteren darauf hin, dass paranoide Patienten vor allem Navigationsdefizite im egozentrischen Raum aufwiesen, während desorganisierte Patienten eher durch Schwierigkeiten im allozentrischen Raum charakterisiert waren. Die parietalen 
Volumina unterschieden sich zwischen den schizophrenen Subgruppen nicht signifikant, allerdings zeigten sich Effekte im globalen Cortexvolumen. Aufgrund der nur geringen Zellenbesetzungen sollten diese Befunde jedoch mit Vorsicht interpretiert werden.

Schließlich zeigte sich im Rahmen der klassischen neuropsychologischen Testung, dass schizophrene Probanden starke Defizite in mnestischen sowie psychomotorischen Funktionen aufwiesen.

Insgesamt liefert die vorliegende Arbeit Belege für defizitäre ego- und allozentrische Navigationsleistungen bei der Schizophrenie sowie eine diesbezügliche pathologische Involvierung parietaler Cortexareale. 


\section{Theoretischer Hintergrund}

Ziel der vorliegenden Arbeit ist es, mit komplexen visuell-räumlichen Navigationsprozessen defizitäre neuropsychologische Testleistungen schizophrener Patienten zu erfassen sowie deren Assoziation zu (dysfunktionalen) neuronalen Korrelaten - hier dem Parietallappen - herzustellen.

Bevor auf die spezifische Themenstellung eingegangen wird und die Versuchshypothesen abgeleitet werden, erfolgt im ersten Abschnitt der Einleitung zunächst eine allgemeine Charakterisierung des Symptombildes anhand psychopathologischer und neuropsychologischer Merkmale sowie die Darstellung der damit assoziierten neuronalen Grundlagen. Im Anschluss wird im zweiten Teil mit der visuell-räumlichen Navigationsleistung der Probanden eine primär ,parietale“ Hirnfunktion skizziert, die in der vorliegenden Arbeit mit Hilfe eines neuen Paradigmas objektiviert werden soll: Der „Virtuellen Realität“.

\subsection{Allgemeiner Überblick}

\subsubsection{Die Phänomenologie der Schizophrenie: Eine Einführung}

„Schizophrenia is one of the most devasting disorders in psychiatry, and it affects the highest mental functions (e.g. thinking, feeling, and perceiving).”

(Peled, 1999, S. 245)

Ungefähr ein Prozent der Weltbevölkerung ist mit der Schizophrenie von einem der schwersten psychiatrischen Krankheitsbilder betroffen ${ }^{1}$. Das Symptomprofil der Schizophrenie umfasst eine Vielzahl an psychopathologischen Symptomen und neurokognitiven Defiziten, die starke Begrenzungen für die intellektuelle, soziale und berufliche Weiterentwicklung darstellen und eine hohe Belastung für Patienten und Angehörige implizieren (vgl. Goldberg \& Seidman, 1991; Watzl \& Rist, 1997; Hahlweg, 2000).

Als Folge leiden schizophrene Patienten häufig unter depressiven Verstimmungen mit Gefühlen der Hilf- und Hoffnungslosigkeit und Furcht vor dem geistigen Verfall. Darüber hinaus besteht häufig eine komorbide Alkohol- und Drogenabhängigkeit, zahlreiche (unfreiwillige) Hospitalisierungen sind die Regel. Häufige Folge der vielfältigen emotionalen und sozialen Probleme ist die Stigmatisierung der Patienten; es erfolgt eine

\footnotetext{
${ }^{1}$ Die Prävalenz der Schizophrenie beträgt in der Weltbevölkerung circa $1 \%$ und gilt als kulturunabhängig. Die Mehrzahl der epidemiologischen Studien berichtet recht homogene und stabile Inzidenzraten unabhängig von ökonomischen, kulturellen oder ethnischen Einflussfaktoren (Watzl \& Rist, 1997; Hahlweg, 2000).
} 
Ausgrenzung aus der beruflichen und sozialen Gesellschaft (Hambrecht \& Hafner, 1996; Jeste et al., 1996; Jablensky, 2000; Davison \& Neale, 2002; Pompili et al.; 2004; Payne et al., 2006).

Der hohe Leidensdruck schizophrener Patienten schlägt sich auch in einer drastisch erhöhten Suizidalität nieder: Bis zu $50 \%$ der Betroffenen vollziehen einen Selbstmordversuch, circa 10\% sind dabei „erfolgreich“ (Jablensky, 2000; Pompili et al., 2004; Palmer, 2005). Insgesamt ist das Suizidrisiko im Vergleich zur Normalbevölkerung 8,5-fach erhöht (Harris \& Barraclough, 1997), wobei vor allem die ersten Jahre nach Diagnosestellung mit einer hohen Suizidgefahr behaftet sind (Jobe \& Harrow, 2005; Palmer, 2005; Payne et al., 2006).

Bei der Schizophrenie handelt es sich folglich um eine Krankheit mit hohem Zerstörungspotential für das Leben der Betroffenen. Im Folgenden sollen die wichtigsten Befunde zu Psychopathologie, Neuropsychologie und zu Grunde liegender Neurobiologie der Schizophrenie kurz skizziert werden. ${ }^{2}$

\subsubsection{Die Psychopathologie der Schizophrenie}

„My whole mental power has disappeared, I have sunk intellectually below the level of a beast..."

\section{(a patient with schizophrenia, Kraepelin, 1919, zitiert nach O’Carroll, 2000, S. 161)}

Bei der „Schizophrenie“ handelt es sich nicht um eine einheitliche Störung, sondern um ein heterogenes Krankheitskonzept, das eine Vielzahl an unterschiedlichen Symptomen in sich vereint. Das Klassifikationsschema DSM-IV (APA, 1994) unterscheidet fünf zentrale Symptomdimensionen:

1) Wahn

2) Halluzinationen

3) Desorganisierte Sprechweise

4) Grob desorganisiertes oder katatones Verhalten

5) Negative Symptomatik, wie flacher Affekt, Alogie oder Willensschwäche

\footnotetext{
${ }^{2}$ Für einen umfassenden Überblick über Epidemiologie, Nosologie/Phänomenologie, Diagnostik, Klassifikation und Verlauf schizophrener Störungen, neuropsychologische, psychophysiologische \& hirnanatomische Anomalien, psychologische/biologische/multifaktorielle Ätiologietheorien, Versuche der Subtypisierung, pharmakotherapeutische, psychotherapeutische, psychosoziale und rehabilitative Interventionensprinzipien und Rückfallprophylaxe sowie deren Effizienzbewertung/Evaluation siehe klassische Überblicksartikel von Crow \& Johnstone, 1987b; Watzl \& Rist, 1997; Hahlweg, 2000; Helmchen et al., 2000; Pearlson, 2000.
} 
Das mit Abstand häufigste Wahnerlebnis schizophrener Patienten ist der Verfolgungswahn, gefolgt vom Beziehungswahn und den Gefühlen, kontrolliert zu werden oder fremde Gedanken lesen zu können. Akustische Halluzinationen sind die häufigsten Formen von Halluzinationen - sie treten bei circa $70 \%$ der Patienten auf - gefolgt von der Wahrnehmung kommentierender oder dialogisierender Stimmen. Die desorganisierte Sprechweise äußert sich vor allem durch gelockerte Assziationen, Inkohärenz im Denken, Zerfahrenheit oder „Danebenreden“; desorganisieretes Verhalten vor allem im sozialen und sexuellen Bereich, zum Beispiel in Form von stereotypen Handlungen. Die häufigsten negativen Symptome sind Affektverflachung, Apathie, Alogie und Anhedonie.

Tabelle 1 gibt einen weiteren Überblick über die mögliche Ausgestaltung der schizophrenen Symptomkategorien (vgl. Watzl \& Rist, 1997; Davison \& Neale, 2002).

\section{Tabelle 1: $\quad$ Symptomdimensionen der Schizophrenie}

\begin{tabular}{|c|c|c|}
\hline $\begin{array}{l}\frac{\text { 1) Wahn }}{\text { (inhaltliche Denkstörungen) }} \\
\rightarrow \text { Verfolgungs-, Eifersuchts-, } \\
\text { Schuld-, Kontroll-, Größen-, } \\
\text { Beziehungs-, } \\
\text { Beeinflussungswahn; } \\
\text { Grandiosität } \\
\rightarrow \text { Wahnwahrnehmungen } \\
\rightarrow \text { Körperliche Passivität } \\
\rightarrow \text { Gedankeneingebung, - } \\
\text { ausbreitung, -entzug; } \\
\text { "Gemachte“ Gefühle und } \\
\text { Handlungen }\end{array}$ & $\begin{array}{l}\text { 2) Halluzinationen } \\
\rightarrow \text { Halluzinationen jeglicher } \\
\text { Sinnesmodalität } \\
\rightarrow \text { am häufigsten: akustische } \\
\text { Halluzinationen; Gedankenlautwerden, } \\
\text { streitende, dialogische, } \\
\text { kommentierende Stimmen }\end{array}$ & $\begin{array}{l}\frac{\text { 3) Desorganisierte }}{\text { Sprechweise }} \\
\underline{\text { (formale Denkstörungen) }} \\
\text { gelockerte Assoziationen; } \\
\text { Inkohärenz; } \\
\text { Gedankenabreißen; } \\
\text { Einschiebungen in den } \\
\text { Gedankenfluss; Entgleisen; } \\
\text { Zerfahrenheit oder } \\
\text { Danebenreden; } \\
\text { Neologismen }\end{array}$ \\
\hline $\begin{array}{l}\text { 4) grob desorganisiertes, } \\
\text { bizarres Verhalten } \\
\rightarrow \text { Kleidung, Erscheinungsbild; } \\
\quad \text { soziales/sexuelles Verhalten; } \\
\text { aggressiv-agitiertes, } \\
\text { stereotypes Verhalten } \\
\rightarrow \text { häufig auch Grimassieren; } \\
\quad \text { Manierismen }\end{array}$ & $\begin{array}{l}\text { 5) Negative Symptome } \\
\text { 1) Flacher und inadäquater Affekt } \\
\rightarrow \text { eingeschränkte Mimik, eingeschränkte } \\
\quad \begin{array}{l}\text { Spontanbewegungen; } \\
\text { geringer Blickkontakt; }\end{array} \\
\text { mangelnde Stimmodulation } \\
\text { 2) Anhedonie und sozialer Rückzug } \\
\rightarrow \rightarrow \text { mangelndes Interesse an Freizeit- } \\
\text { aktivitäten, sexuellen Kontakten } \\
\text { 3) Alogie, Sprachverarmung } \\
\rightarrow \rightarrow \text { Sprachverarmung } \\
\text { (quantitativ und qualitativ) } \\
\text { 4) Antriebs-, Willensschwäche Apathie } \\
\rightarrow \text { mangelnde Energie bezügl. Pflege und } \\
\begin{array}{l}\text { Hygiene; Unstetigkeit in Arbeit und } \\
\text { Schule; körperliche Energielosigkeit; } \\
\text { hohe Passivität }\end{array}\end{array}$ & $\begin{array}{l}\text { 6) Katatone Symptome } \\
\text { T Erregung, Haltungs- } \\
\text { stereotypien; } \\
\text { wächserne Biegsamkeit; } \\
\text { Negativismus, Mutismus; } \\
\text { Stupor }\end{array}$ \\
\hline
\end{tabular}


Weist ein Patient mindestens zwei der aufgeführten Symptome ${ }^{3}$ für einen erheblichen Teil einer Zeitspanne von einem Monat auf und halten Zeichen des Störungsbildes insgesamt für mindestens sechs Momate an, so erhält er gemäß DSM-IV (APA, 1994) die Diagnose einer „Schizophrenie“. Dabei erfolgt die Diagnose stets aufgrund individueller Symptomcluster, da bis heute trotz intensiver Bemühungen kein pathognomisches „Kernsymptom“ bestimmt werden konnte, welches alle schizophrenen Patienten miteinander verbindet (vgl. Pearlson, 2000).

Im Anschluss an die Hauptdiagnose erfolgt die Zuordnung der Patienten zu einer der fünf diagnostischen Subkategorien: Patienten, bei denen Symptome einer Positivsymptomatik, wie Wahnvorstellungen und Halluzinationen, im Vordergrund stehen, gelten als Vertreter des paranoiden Subtypus, während das primäre Vorliegen von bizarren Verhaltensweisen, formalen (desorganisierten) Denk- und Sprachstörungen sowie von negativen Symptomen, wie Affektverflachung, Alogie, Anhedonie und Apathie, zu der Klassifikation eines „desorganisierten“ beziehungsweise „hebephrenen“ Subtypus führt. Die Kategorie der „undifferenzierten“ Schizophrenie repräsentiert einen Mischtypus, in dem sich verschiedene Symptome des paranoiden und desorganisierten Krankheitsbildes präsentieren, ohne jedoch das jeweilige Vollbild zu erfüllen. Zwei weitere diagnostische Kategorien ergeben sich aus dem Vorliegen katatoner Symptome (der „katatone“ Subtyp) sowie aus dem häufig auftretenden klinischen Residualzustand („residualer“ Subtyp).

Es gibt zunehmende Bemühungen, die schizophrenen Subgruppen neben phänomenologischen Charakteristika auch durch differentielle neuropsychologische und neuroanatomische Profile zu charakterisieren (z.B. Goldstein \& Tsuang 1988; Levin \& Yurgelun-Todd, 1989; Seltzer et al., 1997; Peled, 1999; Claussen, 2004). Erste Arbeiten sprechen für differentielle Effekte zum Beispiel im Bereich expliziter Gedächtnisfunktionen, die vor allem bei desorganisierten Patienten defizitär zu sein scheinen (Höschel et al., 1998; Weniger et al., 2004a) oder bei der Gesichtererkennung, in der residuale Patienten weniger eingeschränkt sind als desorganisierte oder paranoide Patienten (Weniger et al., 2004b).

Wie unter 5.4. noch genauer erläutert wird, ist der Versuch, das heterogene schizophrene Zustandsbild in distinkte Subtypen mit charakteristischen Symptomprofilen zu gliedern ein wesentlicher Focus der derzeitigen Schizophrenieforschung. Faktorenanalytische Studien weisen auf das Vorliegen von mindestens drei Symptomdimensionen, der „Positivsymptomatik“, der „Negativsymptomatik“ und der „Desorganisation“, hin (vgl.

\footnotetext{
3 Nur ein Kriterium ist erforderlich, wenn der Wahn bizarr ist, oder wenn die Halluzinationen aus kommentierenden/diskutierenden Stimmen bestehen.
} 
Liddle, 1987; Höschel et al., 1998). Darüber hinaus postulieren Peralta et al. (1994) mit der „Beziehungsdimension“ einen vierten Faktor.

\subsubsection{Die Neuropsychologie der Schizophrenie}

"None of the confidence intervals for mean effect sizes in the above domains included zero, which is to say, no behavioral domain tested is spared by the illness"

(Snitz et al., 2005, S. 179)

Eine große Zahl an Arbeiten konnte neben psychopathologischen Merkmalen charakteristische neurokognitive Defizite der Schizophrenie nachweisen (vgl. Goldstein, 1986; Blanchard \& Neale, 1994; Sanfilipo et al., 2002; Zihl, 2004d). Mit einer allgemeinen Intelligenzminderung und Defiziten in den Bereichen Aufmerksamkeit, Wahrnehmung, Lernen und Gedächtnis, exekutive Funktionen, Abstraktion, Problemlösen, (visuellräumliche) Informationsverarbeitung, Sprachverarbeitung und Sprachproduktion sowie Visuo- und Psychomotorik sind eine Vielzahl an kognitiven Funktionsbereichen betroffen. Das neuropsychologische Profil schizophrener Patienten ist auf globalem Niveau beeinträchtigt (für einen Überblick siehe auch Goldberg \& Weinberger, 1986; Watzl \& Rist, 1997; Heinrich \& Zakzanis, 1998; Rund, 1998; O’Carroll, 2000).

Unklar ist allerdings, ob sich über die globalen, unspezifischen Leistungsminderungen hinaus besonders ausgeprägte, differentielle Defizite beobachten lassen, die sich aus dem globalen Defektprofil abheben. Vor allem (explizite) Lern- und (Arbeits-) Gedächtnisfunktionen (Saykin et al., 1991; Saykin et al., 1994; Goldman-Rakic, 1994; Tek et al., 2002; Kindermann et al., 2004) sowie Aufmerksamkeitsprozesse (McGhie \& Chapman, 1961; Cornblatt \& Kelip, 1994; Cornblatt \& Malhotra, 2001; Salgado-Pineda et al., 2003) und exekutive Funktionen (Nelson et al., 1998; Snitz et al., 2005) wurden als überdurchschnittlich stark betroffen diskutiert. Die Befunde verschiedener Arbeitsgruppen blieben jedoch inkonsistent (vgl. Blanchard \& Neale, 1994; Rund, 1998; Heinrichs \& Zakzanis, 1998; O’Carroll, 2000; Zihl, 2004d). Auch die Suche nach neurokognitiven „Kardinalsymptomen“ der Schizophrenie, also nach (kognitiven) Defiziten, die bei allen Patienten objektivierbar sind, blieb ergebnislos (Watzl \& Rist, 1997; O’Carroll, 2000). Bisher ist es nicht gelungen, ein schizophrenie-spezifisches Profil oder ein pathognomisches Kernsymptom zu definieren, welches schizophrene Patienten eindeutig zu identifizieren und von anderen psychiatrischen Störungsgruppen abzugrenzen vermag. Trotzdem implizieren neuropsychologische Testprofile einen großen diagnostischen Nutzen, um Aussagen über kognitive Einschränkungen und die Alltagsfähigkeit der 
Patienten treffen zu können. Darüber hinaus besteht ihr wesentliches Potential in der Möglichkeit, Defizite bestimmter Funktionsbereiche mit spezifischen neuronalen Korrelaten zu assoziieren (vgl. Goldstein, 1986; Goldberg \& Seidman, 1991; Levin \& Yurgelun-Todd, 1989; Kolb \& Whishaw, 1996). Im Folgenden sollen die wichtigsten Befunde kurz skizziert werden. ${ }^{4}$

\subsubsection{Die Neurobiologie der Schizophrenie}

“...we propose that schizophrenia is best viewed as a brain disorder...”

(Shenton et al., 2001, S. 6)

Für viele der beschriebenen psychopathologischen und neuropsychologischen Merkmale konnte bereits eine neuronanatomische Lokalisation, vorwiegend in frontalen und temporalen Arealen ausgemacht werden - die Schizophrenie wird allgemeinhin als „,brain disorder" verstanden (Heinrichs \& Zakzanis, 1998; Harrison, 1999; Goldberg \& Seidman, 1991; McCarley et al., 1999; Pearlson, 2000; Shenton et al., 2001; Pantelis et al., 2003; Cho et al., 2004).

\subsubsection{Der Frontallappen}

„...the frontal cortex which is especially well developed in man stands in close relation to his higher intellecutal abilities and these are the faculties which in our patients invariably suffer profound loss..."

(Kraepelin, 1919 zitiert nach Goldberg \& Seidman, 1991, S. 553)

Der Frontallappen (FL) und insbesondere sein dorsolateral-präfrontales Subareal (DLPFC) werden aufgrund vielfältiger Befunde häufig als das neuronale Korrelat der Schizophrenie angesehen: So weisen schizophrene Patienten Defizite in typischen „(prä-)frontalen Funktionen“ wie Aufmerksamkeis- (Maruff et al., 1995; Salgado-Pineda et al., 2003; Zihl, 2004b,d) und Arbeitsgedächtnisprozessen ${ }^{5}$ (Park \& Holzman, 1992; Park et al., 1995; Carter et al., 1996; Fleming et al., 1997; Goldman-Rakic \& Selemon, 1997; Tek et al.,

\footnotetext{
${ }^{4}$ In der vorliegenden Arbeit werden ausschließlich die Befunde zu cortikalen Arealen - dem Frontal-, Temporal- und Parietallappen - dargestellt. Für einen weiterführenden Überblick bezüglich Ventrikelerweiterungen \& subkortikalen Strukturanomalien siehe McCarley et al., 1999; Wright et al., 2000 \& Shenton et al., 2001.

${ }^{5}$ Der präfrontale Cortex (PFC) und im Besonderen der dorsolaterale PFC (DLPFC) werden häufig als neuronales Synonym von Aufmerksamkeits- und Arbeitsgedächtnisprozessen verstanden (z.B. Yang et al., 1999; Cho et al., 2004; Kindermann et al., 2004; Zihl, 2004b,d) - wie unter 1.1.4.3.3. noch genauer diskutiert wird, ist der (DL)PFC zwar ein wichtiger Bestandteil dieser kognitiven Funktionen, stellt allerdings nur einen Teil eines übergordneten (frontoparietalen) Aufmerksamkeits- und Arbeitsgedächtnis-Netzwerks dar.
} 
2002; Jansma et al., 2004; Tan et al., 2005) sowie in exekutiven Funktionen (Hutton et al., 1998; Zihl, 2004c,d; Badcock et al., 2005; Van Beilen et al., 2005) auf. Der FL repräsentiert zudem als onto- und phylogenetisch jüngstes Cortexareal jene höchsten kognitiven Funktionen des Menschen, die bei der Schizophrenie in besonderem Maße beeinträchtigt sind (vgl. Goldberg \& Seidman, 1991; Shenton et al., 2001).

Neben den neuropsychologischen Testprofilen weist auch die große Ähnlichkeit des Symptombilds zu dem von Frontallappenläsionen auf eine Involvierung des FL hin (vgl. Goldberg \& Weinberger, 1986; Levin \& Yurgelun-Todd, 1989; Kolb \& Whishaw, 1996). Zudem besteht ein Zusammenhang zu psychopathologischen Symptomen: Insbesondere die Negativsymptomatik der Schizophrenie konnte mit Anomalien im FL assoziiert werden (Zec \& Weinberger, 1986; Buchanan et al., 1993, 1998; Raja, 1996; Andreasen et al., 1997; McCarley et al., 1999; Sanfilipo et al., 2002; Ho et al., 2003), während seltener auch ein Zusammenhang $\mathrm{zu}$ positiven Schizophreniesymptomen festgestellt wurde (vgl. Cleghorn et al., 1990; Spence et al., 1997).

Neben psychopathologischen und neuropsychologischen Befunden weist schließlich auch eine große neuroanatomische Datenbasis auf die Pathologie des FL (und insbesondere des DLPFC) hin - auch wenn es sich insgesamt um eher subtile Effekte handelt (für einen Überblick siehe Shenton et al., 2001; Wright et al., 2000; McCarley et al., 1999; Cho et al., 2004).

\subsubsection{Der Temporallappen}

\section{"Temporal lobe structures and their projections have been suspected to be the seat of pathophysiology of psychosis since Kreaepelin (1919)...”}

(Rajarethinam et al., 2001, S. 80)

Neben frontalen wurden auch temporale Cortexareale häufig als Sitz der schizophrenen Psychopathologie angesehen - viele Studien konnten hier große (Lawrie \& Abukmeil, 1998; Harrison, 1999; Shenton et al., 2001; Cho et al., 2004; Abbott \& Bustillo, 2006), wenn nicht sogar die größten (Chua \& McKenna, 1995; Baumann \& Bogerts, 1999; McCarley et al., 1999; Gur et al., 2000; Wright et al., 2000; Pantelis et al., 2003) cortikalen Anomalien feststellen, wobei sich die deutlichsten Effekte in temporolimbischen Arealen (Tamminga et al., 1992; Nelson et al., 1998; Goldstein et al., 1999) sowie im superioren temporalen Gyrus (STG, McCarley et al., 1999; Sigmundsson et al., 2001; Sweet et al., 2003) zeigten. 
Defizite in Lern- und Gedächtnisfunktionen sind mit Dysfunktionen in medialen Temporallappenstrukturen - insbesondere dem Hippocampus - assoziiert (vgl. Nelson et al., 1998; Gur et al., 2000; Sanfilipo et al., 2002; Szeszko et al., 2002; Wood et al., 2002; Sweatt, 2004), Defizite in der Verarbeitung emotionalen Materials werden mit einer Dysfunktion amygdaloider Strukturen (vgl Nelson et al., 1998; Exner et al., 2004) und Einbußen in der auditorischen und semantischen Sprachverarbeitung primär mit Anomalien des auditorischen Assoziationscortex in Verbindung gebracht (Petty et al., 1995; Pearlson et al., 1997; Sweet et al., 2003).

Zudem sind temporale Areale (Zec \& Weinberger, 1986; Bogerts et al., 1993; Shergill et al., 2001; Shapleske et al., 2002) und insbesondere der STG (Barta et al., 1990; Menon et al., 1995; Petty et al., 1995; Marsh et al., 1999; Harrison, 1999; Gur et al., 2000; Onitsuka et al., 2004) an der Genese der schizophrenen Positivsymptomatik maßgeblich beteiligt. ${ }^{6}$

\subsubsection{Der Parietallappen}

"...the evidence strongly implicates the involvement of the $P L$ in the presentation of schizophrenia...”

(Danckert et al., 2004, S. 256)

Wie aus der bisherigen Darstellung ersichtlich, konnten viele Symptome der Schizophrenie mit frontalen und temporalen Cortexanomalien in Verbindung gebracht werden. Im Gegensatz dazu wurde der Parietallappen bei der Diskussion um die neurobiologische Schizophreniegenese weitgehend vernachlässigt. Und das, obwohl es bereits eine Vielzahl an Hinweisen auf seine psychopathologische Beteiligung gibt, wie der folgende Überblick zeigt (vgl. Goldstein et al., 1999; Frederikse et al., 2000; Shenton et al., 2001; Danckert et al., 2004):

\subsection{Neuroanatomie: Parietale Anomalien}

\subsection{Empirische Befunde}

Wie aus Tabelle 2 ersichtlich, spricht eine Reihe empirischer Arbeiten für eine pathologische Beteiligung parietaler Cortexareale bei der Schizophrenie: Insbesondere Volumenreduktionen (und selten auch -zunahmen) konnten neben funktionellen Hypo- und Hyperaktivitäten in den verschiedensten parietalen Subarealen beobachtet werden.

\footnotetext{
${ }^{6}$ Dafür sprechen auch die Beobachtungen, dass Temporallappenepileptiker häufig unter positiven Symptomen wie Halluzinationen leiden (Flor-Henry, 1969) und dass sich diese durch elektrische Stimulation temporaler Areale hervorrufen lassen (Penfield \& Perot, 1963; Bancaud et al., 1994).
} 
Tabelle 2: $\quad$ Strukturelle und funktionelle Anomalien des Parietallappens bei der Schizophrenie ${ }^{7}$

\begin{tabular}{|c|c|c|c|}
\hline$\underline{\text { Studien }}$ & Befunde/ Anmerkungen & $\underline{\text { Studien }}$ & $\frac{\text { Befunde/ }}{\text { Anmerkungen }}$ \\
\hline $\begin{array}{l}\text { Shenton et al., } 2001 \\
\text { (Review, } \mathrm{n}=193 \text { ) }\end{array}$ & $\begin{array}{l}\text { - globale PL-Volumen- } \\
\text { reduktion in } 60 \% \text { der Studien } \\
\text { - stärkere Effekte als für d. FL } \\
\text { - stärkste Reduktionen im IPL }\end{array}$ & $\begin{array}{l}\text { McCarley et al., } 1999 \\
\text { (Review, } \mathrm{n}=118 \text { ) }\end{array}$ & $\begin{array}{l}\text { - nur schwache Effekte: } \\
44 \% \text { der Studien finden } \\
\text { PL-Volumen- } \\
\text { reduktionen } \\
\text { - keine Unterteilung in } \\
\text { parietale Subareale }\end{array}$ \\
\hline $\begin{array}{l}\text { Pearlson \& Marsh, } \\
1999 \\
\text { (selektiver Review) }\end{array}$ & $\begin{array}{l}\text { - globale PL-Volumen- } \\
\text { reduktion um ca. } 10-15 \% \\
\text { - betont Rolle des HASC (IPL) }\end{array}$ & & \\
\hline $\begin{array}{l}\text { Thompson et al., } 2001 \\
\text { Gogtay et al., } 2003 \\
\text { Lieberman et al., } 2005\end{array}$ & $\begin{array}{l}\text { - globale PL- } \\
\text { Volumenreduktion } \\
\text { - Thompson: früheste patho- } \\
\text { logische Anomalien im PL } \\
\text { (noch vor FL, TL) } \\
\text { - Gogtay \& Lieberman: größte } \\
\text { Effekte in PL (vs. FL, TL) }\end{array}$ & $\begin{array}{l}\text { McGilchrist, } 1993 \\
\text { Schlaepfer et al., } 1994 \\
\text { Goldstein et al., } 1999 \\
\text { Frederikse et al., } 2000 \\
\text { Wilke et al., 2001 } \\
\text { Kubicki et al., } 2002 \\
\text { Buchanan et al., } 2004 \\
\text { Maruff et al., 2005 }\end{array}$ & $\begin{array}{l}\text { Volumenreduktionen } \\
\text { im IPL (Areae 39, 40) }\end{array}$ \\
\hline $\begin{array}{l}\text { Frederikse et al., } 2000 \\
\text { Niznikiewicz et al., } \\
2000 \\
\text { Buchanan et al., 2004b }\end{array}$ & $\begin{array}{l}\text { - Umkehr der normalen } \\
\text { Asymmetrie des IPL: re > li }\end{array}$ & Kirkpatrick et al., 1999 & $\begin{array}{l}\text { - pathologisch erhöhte } \\
\text { Zelldichte im IPL }\end{array}$ \\
\hline Pol et al., 2001 & $\begin{array}{l}\text { - Volumenreduktionen im IPL } \\
\text { und im precuneus }\end{array}$ & Shapleske et al., 2002 & $\begin{array}{l}\text { - Volumenreduktion im } \\
\text { precuneus/medialen PL }\end{array}$ \\
\hline $\begin{array}{l}\text { Cannon et al., 2001, } \\
2002\end{array}$ & $\begin{array}{l}\text { - Volumenreduktionen im } \\
\text { superioren PL }\end{array}$ & $\begin{array}{l}\text { Shapleske et al., } 2002 \\
\text { Salgado-Pineda et al., } \\
2003\end{array}$ & $\begin{array}{l}\text { - Volumenerhöhungen } \\
\text { im IPL (Area 40) }\end{array}$ \\
\hline $\begin{array}{l}\text { Marsh et al., 1997, } \\
1999\end{array}$ & $\begin{array}{l}\text { - frontoparietale } \\
\text { Volumenreduktion }\end{array}$ & Foong et al., 2001 & $\begin{array}{l}\text { - Volumenreduktionen } \\
\text { im parieto-occipitalen } \\
\text { Cortex }\end{array}$ \\
\hline $\begin{array}{l}\text { Abbott \& Bustillo, } \\
2006 \\
(\text { Review, } n=20)\end{array}$ & $\begin{array}{l}\text { - Hinweise auf funktionelle } \\
\text { Anomalien im PL }\end{array}$ & $\begin{array}{l}\text { Kishimoto et al., } 1987 \\
\text { Andreasen et al., } 1997 \\
\text { Kindermann et al., } 2004\end{array}$ & $\begin{array}{l}\text { - Hypoaktivität des } \\
\text { globalen PL }\end{array}$ \\
\hline $\begin{array}{l}\text { Cleghorn et al., } 1989 \\
\text { Tamminga et al., } 1992 \\
\text { Schultz et al., } 2002 \\
\text { Streit et al., 2001 }\end{array}$ & - Hypoaktivität des IPL & Tendolkar et al., 2005 & $\begin{array}{l}\text { - Anormale EKP- } \\
\text { Muster über centro- } \\
\text { parietalen Arealen } \\
\text { (primär IPL) }\end{array}$ \\
\hline Quintana et al., 2003 & - Hyperaktivität des PPC & Paulus et al., 2002 & $\begin{array}{l}\text { - Hyperaktivität des } \\
\text { Gyrus postcentralis und } \\
\text { des IPL }\end{array}$ \\
\hline Königsberg et al., 2005 & $\begin{array}{l}\text { - Hypoaktivität des } \\
\text { intraparietalen Cortex }\end{array}$ & Kindermann et al., 2004 & - Hyperaktivität des IPL \\
\hline Kim et al., 2003 & $\begin{array}{l}\text { - Funktionelle Diskonnektion } \\
\text { zwischen PFC und IPL }\end{array}$ & & \\
\hline
\end{tabular}

PL = Parietallappen; IPL = inferiorer Parietallappen; PPC = posterior-parietaler Cortex; FL = Frontallappen; $\mathrm{PFC}=$ präfrontaler Cortex; $\mathrm{TL}=$ Temporallappen; HASC $=$ heteromodaler Assoziationscortices; EKP = Ereigniskorrelierte Potentiale.

\footnotetext{
${ }^{7}$ Eine Auflistung der verwendeten Abkürzungen findet sich unter 7.1..
} 
Aus Tabelle 2 wird ersichtlich, dass eine recht große Datenbasis auf die Pathologie parietaler Areale innerhalb des schizophrenen Krankheitsbildes hinweist. Anzumerken ist allerdings, dass einige der referierten Befunde eher ein „Nebenprodukt“ der jeweiligen Arbeiten darstellen. Anomalien in parietalen (Sub-)Strukturen wurden zwar häufig dokumentiert, nahmen jedoch in der weiteren Diskussion meist keinen nennenswerten Stellenwert ein.

\subsection{Pearlsons Theorie heteromodaler Assoziationscortices}

"Schizophrenia may be thought of as a disease of the most uniquely human portions of the brain and in fact may be a purely human disease..."

(Pearlson, 1996, S. 12)

Die empirische Befundlage einer parietalen Pathologie deckt sich mit einer prominenten Theorie von Pearlson et al. (1996, 1999, 2000), der in Anlehnung an Mesulams funktionelle Cortexkartierung $(1985,1990,1998)$ von einer primären Pathologie heteromodaler Assoziationscortices (HASC) bei der Schizophrenie ausgeht. HASC-Areale sind in komplexen Netzwerken organisiert, zeichnen sich durch starke Interkonnektivität und Lateralisierung aus, treten in der onto- und phylogenetischen Entwicklung erst relativ spät auf und repräsentieren mit multimodalen Kognitionen die höchsten und „menschlichsten“ cortikalen Funktionen. Funktionen, die bei der Schizophrenie in besonderem Maße betroffen sind. Aufgrund dieser Charakteristika scheinen Areale des heteromodalen Assoziationscortex prädestiniert, um eine neuronale Antwort auf das komplexe psychopathologische Symptombild zu geben (Pearlson, 1996, 1999, 2000; vgl. auch Ross \& Pearlson, 1996; McCarley et al., 1999; Wilke et al., 2001; Buchanan et al., 1998, 2004b).

Drei heteromodale Assoziationscortices sind laut Pearlson (1996, 1999, 2000) von Relevanz: Der (dorsolaterale) präfrontale Cortex (DLPFC), der für die Genese der Negativsymptomatik und neuropsychologische Defizite in exekutiven Funktionen, Arbeitsgedächtnis- und Aufmerksamkeitsprozessen verantwortlich ist, der superiore temporale Gyrus (STG), der primär an der Entstehung der Positivsymptomatik beteiligt ist und zudem der inferior parietale Cortex (IPL), der vor allem mit Defiziten in visuellräumlichen (Aufmerksamkeits-) Funktionen in Verbindung steht und zudem an der multimodalen Integration visuell-räumlicher, propriozeptiver und motorischer Reize 
wesentlich beteiligt ist (vgl. auch Andersen, 1997; Save \& Poucet, 2000; Culham \& Kanwisher, 2001; MacDonald \& Paus, 2003).

Wie aus der bisherigen Diskussion ersichtlich, spricht die Empirie deutlich für eine (primäre) psychopathologische Beteiligung heteromodaler Areale - insbesondere der (DL)PFC und der STG weisen robuste Anomalien auf - und impliziert so auch eine psychopathologische Beteiligung des (inferioren) Parietallappens (vgl. Tabelle 2 und insbesondere Schlaepfer et al., 1994; Sullivan et al., 1998; Baumann \& Bogerts, 1999; Frederikse et al., 2000; Niznikiewicz et al., 2000; Cannon et al., 2001, 2002; Keshavan et al., 2003; Mitelman et al., 2003; Buchanan et al., 2004; Danckert et al., 2004). ${ }^{8}$

\subsection{Psychopathologie: Eine parietale Genese}

„...our study lends...support for the role of the...parietal region in the aetiology of such ,alienation”...“

(Spence et al., 1997, S. 2007)

Zudem scheinen neben temporalen auch parietale Areale an der Genese der schizophrenen Positivsymptomatik beteiligt zu sein: So sind Wahnvorstellungen wie „passivity delusions“ und akustische Halluzinationen nach dem „Frith-Modell“ (Frith, 1987; Frith et al., 2000) Folge eines (inferior)-parietal vermittelten self-monitoring Defizits, bei dem die Betroffenen unfähig sind, selbst- und fremdgenerierte Kognitionen und Handlungen voneinander zu unterscheiden. Frith (Frith, 1987; Frith et al., 2000) spricht auch von einem parietal vermittelten Defizit im „,sense of agency“, Danckert (2004) von einem „,breakdown of self-monitoring of thoughts and behaviours“. Auf neuronaler Ebene scheint die Positivsymptomatik durch parietale Volumenreduktionen (Maruff et al., 2005) beziehungsweise die Hyperaktivität in inferior-parietalen Cortexarealen repräsentiert zu sein (vgl. Spence et al., 1997; Frith et al., 2000; Shergill et al., 2001).

Eine Vielzahl an Autoren konnte dieses (Daprati et al., 1997; Franck et al., 2001; Farrer \& Frith, 2002; Blakemore \& Frith, 2003; Danckert et al., 2004) oder verwandte (McGuire et al., 1996; Weiller et al., 1996; MacDonald \& Paus, 2003) parietale Ätiologiemodelle der Positivsymptomatik mit Hilfe empirischer Evidenz untermauern. Zudem weisen klinische Daten auf parietale Dysfunktionen im Zusammenhang mit gestörter propriozeptiver Informationsverarbeitung zum Beispiel im Rahmen von Phantomerlebnissen hin (vgl. Spence et al., 1997).

\footnotetext{
${ }^{8}$ Anzumerken ist, dass Pearlson in späteren Arbeiten (1999, 2000) von seiner ursprünglichen These einer exklusiven HASC-Pathologie abkommt und lediglich die primäre Beteiligung heteromodaler Areale postuliert. Dies deckt sich mit der empirischen Evidenz, die auch auf subkortikale Anomalien hinweist (vgl. z.B. Shenton et al., 2001).
} 


\subsection{Neuropsychologie: Defizite in ,parietalen“" Funktionen}

„...the parietal lobe has received very little attention in schizophrenia research, despite the fact that it is a brain region important to processes that are disturbed in schizophrenia such as language, spatial working memory and attention..."

(Shenton et al., 2001, S. 27)

Auch viele der unter 1.1.3. beschriebenen neuropsychologischen Defizite sind mit Dysfunktionen des Parietallappens begründbar (vgl. Frederikse et al., 2000; Danckert et al., 2004). $\mathrm{Zu}$ diesen zählen beispielsweise Leistungseinbußen in visuo- und psychomotorischen Prozessen: Der PL ist an der Auswahl, Planung, Imagination, mentaler Repräsentation, Antizipation und Intiierung zielgerichteten Verhaltens, wie komplexen Motorsequenzen, Augen(folge)bewegungen oder visuell geführtem (Greif-)Verhalten wesentlich beteiligt (Duhamel et al., 1992a,b; Clower et al., 1996; Wolpert et al., 1998) Funktionen, in denen Schizophrene markante Defizite aufweisen (vgl. Sanfilipo et al., 2002; Danckert et al., 2002, 2004).

Des Weiteren registrieren und verarbeiten parietale Cortexareale eine Vielzahl körperbezogener, propriozeptiver Reize und sind so wesentlich an der Generierung des eigenen Körperschemas (vgl. Berlucchi \& Aglioti, 1997; Blakemore \& Frith, 2003; Danckert et al., 2004; Zihl, 2004a) und an der Bildung egozentrischer Repräsentationen (Clower et al., 1996; Wolpert et al., 1998; Culham \& Kanwisher, 2001; MacDonald \& Paus, 2003; Danckert et al., 2004) beteiligt. Wie bereits diskutiert, weisen schizophrene Patienten hier fundamentale Dysfunktionen auf, was unter anderem zur Genese von „passivity delusions“ beiträgt.

Neben egozentrischen sind auch Defizite in exozentrischen Kognitionsleistungen mit parietalen Dysfunktionen assoziiert: So werden die ausgeprägten (visuell-räumlichen) Aufmerksamkeits- und Arbeitsgedächtnisdefizite schizophrener Patienten entsprechend neuerer, multilokalisationistischer Ansätze nicht mehr exklusiv frontalen Arealen sondern der Dysfunktion eines übergeordneten präfrontal-posterior-parietalen Netzwerks zugeschrieben: „...the prefrontal (PFC) and the posterior parietal (PPC) cortices are main components of a distributed neural network for working memory (WM), perceptionaction integration, and goal oriented behavior... “ (Quintana et al., 2003, S. 12; vgl. auch Fleming et al., 1997; Huguelet et al., 2000; Paulus et al., 2002; Schultz et al., 2002; Kim et al., 2003; Kindermann et al., 2004; Koenigsberg et al., 2005; Tan et al., 2005; Tendolkar et al., 2005). Viele der unter 1.1.4.1. aufgeführten Befunde zu defizitären visuell-räumlichen 
Kognitionsleistungen weisen somit auch auf eine Dysfunktion des Parietallappens hin auch wenn dies von den jeweiligen Autoren zugunsten (prä-)frontaler Prozesse nicht immer explizit formuliert worden ist (z.B. Park \& Holzman, 1992; Park et al., 1995; Carter et al., 1996).

Zudem belegt eine Vielzahl klassischer neuropsychologischer Arbeiten (Posner, 1980; Posner \& Petersen, 1990; Büchel et al., 1998; Corbetta, 1998a; Corbetta et al., 1998b; Gitelman et al., 1999; Wojcioulik \& Kanwisher, 1999; Nobre et al., 2000; Goldberg et al., 2002) die Lokalisation visuell-räumlicher Aufmerksamkeits- und Arbeitsgedächtnisprozesse im Parietalcortex (beziehungsweise im fronto-parietalen Netzwerk). So zeigen sich beispielsweise bei Patienten mit parietalen Läsionen mit dem Auftreten eines Neglectoder Balintsyndroms fundamentale Störungen der visuell-räumlichen Aufmerksamkeitsund Informationsverarbeitungsleistung (vgl. Petersen et al., 1989; Kolb \& Whishaw, 1996; Driver \& Mattingley, 1998; Marshall et al., 2002); ähnlich, wie man sie auch bei einem großen Teil der schizophrenen Patienten beobachten kann (vgl. Harvey et al., 1993).

\subsection{Die aktuelle Forschungsmotivation}

Aus der bisherigen Diskussion wird deutlich, dass eine Vielzahl an Befunden auf die Involvierung parietaler Areale bei der Genese der schizophrenen Psychopathologie ${ }^{9}$ hinweist und dass der Parietallappen trotzdem erst selten im Focus wissenschaftlicher Bemühungen stand. Dies soll mit der vorliegenden Arbeit geändert werden:

Auf neuropsychologischer Ebene sollen mit komplexen visuell-räumlichen Navigationsprozessen klassische „parietale“ Hirnfunktionen erfasst und potentielle Dysfunktionen bei der Schizophrenie festgestellt werden. Ergänzend dazu soll mit Hilfe eines MRT-Scans geprüft werden, ob sich die häufig beschriebenen Volumenreduktionen in parietalen (Sub)Arealen objektivieren lassen und ob diese in einem funktionalen Zusammenhang zu der Navigationsleistung stehen.

Im Folgenden wird zunächst das Konzept der visuell-räumlichen Navigation genauer erläutert, bevor im Anschluss das Paradigma der Virtuellen Realität vorgestellt wird, mit der diese multimodalen Kognitionsleistung erfasst werden soll.

\footnotetext{
9 Anzumerken ist, dass unter dem Begriff „Psychopathologie“ im Folgenden nicht nur die unter 1.1.2. subsumierten Krankheitssymptome verstanden werden, sondern im Sinne einer weiteren Definition auch neuropsychologische und neuroanatomische Merkmale der Schizophrenie, die den Focus der vorliegenden Arbeit darstellen.
} 


\subsubsection{Das Konzept der „,visuell-räumlichen Navigation“6}

\subsubsection{Ego- und allozentrische Repräsentationen}

Im Focus der vorliegenden Arbeit stehen visuell-räumliche Navigationsprozesse. Diese werden in der neuropsychologischen Literatur mit Unterscheidungen wie ,route representation“ versus „,survey representation“ (Thorndyke \& Hayes-Roth, 1982; Ruddle et al., 1997; Waller et al., 1998; Mellet et al., 2000a; Shelton \& Gabrieli, 2002), „route knowledge“ versus „,configurational knowledge“ (Gillner \& Mallot, 1998) oder „routebased representation“ versus „map-like representation“ (Aguirre et al., 1998) primär durch die unterschiedlichen mentalen Repräsentationsformen operationalisiert, die der visuellräumlichen Orientierungsfähigkeit zugrunde liegen. Einen Überblick über bisherige Konzepte und den Versuch einer integrativen Taxonomie geben Aguirre \& D'Eposito (1999).

In der vorliegenden Arbeit soll vor allem auf die Terminologie von Tolman et al. (1946, 1947, 1948) Bezug genommen werden, der im Rahmen seiner „cognitive map theory“ egozentrische und allozentrische Repräsentationen unterscheidet, auf denen Navigationsleistungen im extrapersonalen Raum basieren: Egozentrische (d.h. ,auf sich selbst beziehende“, synonym: „body-centered“) Navigation entsteht, wenn eine räumliche Konfiguration $\mathrm{zu}$ erlernen ist, ohne dass charakteristische, externe Hinweisreize - wie Wegmarken - zur Verfügung stehen. Da keine visuellen Reize zur Navigation gegeben sind, müssen in stärkerem Maße propriozeptive Reize herangezogen werden (vgl. Burgess et al., 2001a,b; Weniger \& Irle, 2006). Ein Beispiel für die Entstehung egozentrischer Repräsentationen geben Hartley et al. (2003, S.877): “when the same route is followed over and over again, a representation of the sequence of bodily movements may develop". Allozentrische (d.h. „sich auf die Welt beziehende“, synonym: „world-centered“) Repräsentationen entstehen hingegen, wenn räumliche Konfigurationen unter Einbeziehung von Objekten aus der Umgebung erlernt werden, die „kognitive Landkarte“ also im Wesentlichen über charakteristische Landmarken (wie beispielsweise Bäume, Häuser oder Zäune) erstellt wird (vgl. Kitchin, 1994; Aguirre et al., 1998; Gillner \& Mallot, 1998; Save \& Poucet, 2000). Hartley et al. (2003, S. 877) beschreiben die allozentrische Repräsentation als "global form of representation in which landmark locations are represented in terms of their spatial relationship to one another".

Die Unterscheidung von ego- und allozentrischer Repräsentationen findet sich in den Arbeiten vieler Autoren wieder und weist starke inhaltliche Überschneidungen mit alternativen Konzepten auf (vgl. z.B. Gilmore \& Johnson, 1997; Burgess et al., 2001b, 
Aguirre et al., 1998; Gillner \& Mallot, 1998; Grön et al., 2000; Hartley et al., 2003; Weniger \& Irle, 2006). Tabelle 3 gibt einen Überblick über unterschiedliche Operationalisierungen der visuell-räumlichen Navigation, deren Charakteristika sowie über diskutierte neuronale Korrelate, die im Folgenden noch dargestellt werden.

Tabelle 3: ,Synonyme“, verwandte Begrifflichkeiten, Charakteristika und neuronale Korrelate der visuell-räumlichen Navigation

\begin{tabular}{|c|c|c|c|}
\hline \begin{tabular}{|c|}
$\frac{\text { Mentale }}{\text { Repräsentations }}$ \\
$\underline{\text { formen }}$ \\
\end{tabular} & $\begin{array}{l}\frac{\text { Synonyme, }}{\text { verwandte Begriffe }} \\
\end{array}$ & Charakteristika & $\begin{array}{l}\frac{\text { Diskutierte }}{\text { neuronale }} \\
\underline{\text { Korrelate }} \\
\end{array}$ \\
\hline egozentrisch & $\begin{array}{l}\text { - route-based representation } \\
\text { - body-centered representation } \\
\text { - route knowledge } \\
\text { - action-based representation }\end{array}$ & $\begin{array}{l}\text { - Wissen über bestimmten Weg } \\
\text { - Routenwissen } \\
\text { - abhängig von propriozeptiven } \\
\text { Reizen (z.B. Abfolge von rechts- } \\
\text { links Bewegungen) } \\
\text { - abhängig von der eigenen } \\
\text { (egozentrischen) Perspektive } \\
\text { - prozedural, weniger deklarativ } \\
\text { - beansprucht vor allem KZG } \\
\text { - einfach }\end{array}$ & $\begin{array}{l}\text { parietale Areale: } \\
\text { - posteriorer PL } \\
\text { - inferiorer PL } \\
\text { - superiorer PL } \\
\text { - Precuneusregion }\end{array}$ \\
\hline allozentrisch & $\begin{array}{l}\text { - map-like representation } \\
\text { - world-centered } \\
\text { representation } \\
\text { - configurational knowledge } \\
\text { - cognitive map (Tolman) } \\
\text { - place-based representation }\end{array}$ & $\begin{array}{l}\text { - Wissen über räumliche } \\
\text { Konfigurationen } \\
\text { - Überblickswissen/kognitive } \\
\text { Landkarte } \\
\text { - abhängig von externen (v.a. } \\
\text { visuellen) cues (Landmarken wie } \\
\text { Bäumen, Häusern) } \\
\text { - unabhängig von der eigenen Person } \\
\text { - perspektivunabhängig } \\
\text { - deklarativ } \\
\text { - wird im LZG gespeichert } \\
\text { - komplex }\end{array}$ & $\begin{array}{l}\text { medialer TL: } \\
\text { - Hippocampus } \\
\text { - Gyrus } \\
\text { parahippocampalis }\end{array}$ \\
\hline
\end{tabular}

KZG = Kurzzeitgedächtnis; LZG = Langzeitgedächtnis; PL = Parietallappen.

\subsubsection{Neuronale Korrelate der ego- und allozentrischen Repräsentationen}

Wie aus Tabelle 3 bereits ersichtlich, liegen den unterschiedlichen Repräsentationsformen visuell-räumlicher Navigationsprozesse auch unterschiedliche neuronale Korrelate zugrunde, die die Komponenten eines übergeordneten parieto-hippocampalen beziehungsweise temporo-parietalen Netzwerks darstellen (vgl. Aguirre et al., 1998; 
Maguire et al., 1998a; Mellet et al., 2000; Save \& Poucet, 2000; Burgess et al., 2001b; Guazzelli et al., 2001).

\subsection{Egozentrische Repräsentationen}

Parietale Areale bilden die Grundlage für egozentrische Repräsentationen. Eine Vielzahl an Befunden konvergiert in dem Ergebnis, dass der (posteriore) Parietallappen für eine körperbezogene Navigation wesentlich zu sein scheint (vgl. Berlucchi \& Aglioti, 1997; Maguire et al., 1998a; Snyder et al., 1998; Wolpert et al., 1998; Grön, 2000; Save \& Poucet, 2000; Culham \& Kanwisher, 2001; Guazzelli et al., 2001; Blakemore \& Frith, 2003) - auch wenn verschiedene Autoren den Schwerpunkt bei unterschiedlichen parietalen Subarealen wie der Precuneusregion (Burgess et al., 2001a,b), dem inferioren (Mellet et al., 1996; Maguire et al., 1998a), dem superioren (Grön et al., 2000; Shelton \& Gabrieli, 2002) oder dem posterioren Parietallappen (Aguirre \& D’Eposito, 1999; Guazzelli \& Poucet, 2001) vermuten.

\subsection{Allozentrische Repräsentationen}

Areale des medialen temporalen Cortex repräsentieren hingegen die allozentrische Informationsverarbeitung, also die mentale Repräsentation von Landmarken und deren Relation zueinander (Maguire et al., 1996, 1997; Aguirre et al., 1998; Grön et al., 2000; Guazzelli et al., 2001; Astur et al., 2002, 2004). Vor allem hippocampale Areale werden traditionellerweise als das Korrelat visuell-räumlicher Informationsverarbeitungs-, Lernund Gedächtnisprozesse betrachtet (vgl. Moser et al., 1993; Wood et al., 2002; Szeszko et al., 2003; Sweatt, 2004). Zudem ist der Hippocampus entsprechend der klassischen Theorie von O’Keefe \& Nadel (1978) als neuronales Substrat der „kognitiven Landkarte“ anzusehen (vgl. auch Save \& Poucet, 2000; Guazzelli et al., 2001).

Neuere Arbeiten gehen davon aus, dass neben dem Hippocampus vor allem auch parahippocampale Areale an der allozentrischen Repräsentation topographischer Informationen beiteiligt sind (vgl. z.B. Aguirre et al., 1996; Aguirre et al., 1998; Maguirre et al., 1998; Epstein \& Kanwisher, 1998; Grön et al., 2000; Mellet et al., 2000; Burgess et al., 2001b; Weniger \& Irle, 2006).

\subsection{Das parieto-hippocampale Netzwerk}

Ego- und allozentrische Navigationsprozesse werden auf neuronaler Ebene also durch ein parieto-hippocampales „spatial-cognition“ Netzwerk repräsentiert, in dem temporale Areale primär allozentrische Prozesse realisieren, während parietale Projektionsbahnen vor 
allem die egozentrische Informationsverarbeitung repräsentieren. Entsprechend führen Läsionen der ventralen Bahn ${ }^{10} \mathrm{zu}$ „topographischer Agnosien“, also der Unfähigkeit, Objekte (wie Landmarken) zu erkennen, während dorsale Läsionen vor allem eine egozentrische räumliche Disorientierung bedingen (Levine et al., 1985; Farrell, 1996; Aguirre \& D’Eposito, 1999, Stark et al., 1996; Aguirre et al., 1998).

Trotz vieler empirischer Hinweise zugunsten des parieto-hippocampalen Modells, ist die postulierte funktionelle Trennung der einzelnen Netzwerkkomponenten allerdings zweifelhaft: Zunächst weist eine Vielzahl an Befunden darauf hin, dass auch parietale Areale an allozentrischen Kognitionsleistungen teilhaben (vgl. Duhamel et al., 1992a; Andersen, 1997; Snyder et al., 1998; Save \& Poucet, 2000), zumal mit visuell-räumlichen Aufmerksamkeits- und Arbeitsgedächtnis- sowie psychomotorischen Prozessen wichtige Basisfunktionen der Navigation in parietalen Arealen lokalisiert sind (vgl. 1.1.4.3.3.). Zudem besteht zwischen posterior-parietalen und hippocampalen Arealen eine enge anatomische Interkonnektivität, die auf neuropsychologischer Ebene einen engen funktionellen Zusammenhang vermuten lässt (Aguirre et al., 1998; Save \& Poucet, 2000; Burgess et al., 2001a; Shelton \& Gabrieli, 2002). Darüber hinaus gibt es Hinweise, dass Areale des medialen TL, insbesondere der Gyrus parahippocampalis, neben dem Parietallappen an der Verarbeitung egozentrischer Informationen beteiligt sind (Weniger \& Irle, 2006). Schließlich scheinen sowohl Strukturen des medialen Temporallappens - und insbesondere der Gyrus parahippocampalis (Aguirre et al., 1998; Mellet et al., 2000b; Weniger \& Irle, 2006) - als auch der posterior-parietale (Assoziations-) Cortex (Andersen, 1997; Snyder, 1998; Save \& Poucet, 2000; Burgess, 2001a,b; Guazzelli et al., 2001) an der Integration egozentrischer und allozentrischer Repräsentationen beteiligt zu sein.

Festzuhalten bleibt, dass sowohl posterior-parietale, als auch (para-)hippocampale Cortexstrukturen eine übergeordnete Rolle bei visuell-räumlichen Kognitionsleistungen und somit bei menschlichen Navigationsprozessen besitzen. Oder wie Andersen (1997, S. 1421) es ausdrückt: „The posterior parietal cortex and the hippocampus are areas of the brain considered synonymous with spatial cognition”. Klassische Arbeiten sprechen sich

\footnotetext{
${ }^{10}$ Das Konzept der dorsalen und ventralen Bahn stammt ursprünglich aus dem visuellen System (Mishkin et al., 1983; Ungerleider \& Haxby, 1994). Zwei funktionell divergierende Systeme sind dabei von Relevanz: Die ventrale „WAS“ Bahn dient primär der Objektrekognition (Projektionsbahn: OL-TL-FL-PFC); die dorsale „WO“ Bahn realisiert hingegen die Objektlokalisation (Projektionsbahn: OL-PL-FL-PFC). Dieses neuronale System kann auf visuell-räumliche Kognitionen übertragen werden, indem egozentrische Repräsentationen durch parietale Areale, allozentrische durch medial temporale Areale repräsentiert werden (vgl. O’Donnell et al., 1996; Aguirre et al., 1997, 1998; Mellet et al., 1996, 2000b; Tek et al., 2002).
} 
zwar für eine funktionelle Trennung der Repräsentationsformen und der ihnen zugrunde liegenden Cortexareale aus, diese erscheint aufgrund der aktuellen Befundlage jedoch eher artifiziell. Parietale und (para-)hippocampale Areale scheinen an ego- und allozentrischen Kognitionsleistungen maßgeblich beteiligt zu sein.

Der Focus der vorliegenden Arbeit soll auf der Relevanz parietaler Areale liegen, während die Rolle hippocampaler Areale eher explorativ untersucht wird.

\subsubsection{Objektivierung der visuell-räumlichen Navigation: Die Virtuelle Realität}

Komplexe Navigationsleistungen können, wie im Folgenden dargestellt wird, mit klassischen, zweidimensionalen Testverfahren nur unzureichend abgebildet werden. Daher soll in der vorliegenden Arbeit mit der „Virtuellen Realität“ ein interaktives, multimodales Verfahren zum Einsatz kommen, welches visuell-räumliche Navigationsprozesse mit hoher ökologischer und methodischer Validität zu erfassen vermag.

\subsubsection{Konventionelle neuropsychologische Testverfahren}

Es existiert eine Reihe standardisierter, neuropsychologischer Testverfahren zur Erfassung visuell-räumlicher Informationsverarbeitungsprozesse, wie zum Beispiel der Trail Making Test A und B (TMT A und B, Reitan, 1992) oder der Lern- und Gedächtnis Test-3 (LGT-3, Bäumler, 1974). ${ }^{11}$ Obwohl diese konventionellen ,,paper and pencil“ Tests im klinischen Alltag häufig eingesetzt werden, ist ihre Zweckmäßigkeit fraglich, da sie nur eine geringe ökologische und prognostische Validität aufweisen. Sie sind nur wenig sensitiv, um relevante Defizite aufzuzeigen und Vorhersagen über die Alltagsfähigkeit der Testperson treffen zu können (vgl. Maguire et al., 1996; Kindsmüller et al., 1999; Klinger et al., 2004). Die unzureichenden Validitäten dieser konventionellen Verfahren sind vor allem dadurch zu begründen, dass sie lediglich isolierte, artifizielle Teilbereiche und nicht die synthetischen, integrativen (ego- und allozentrischen) Kognitionsprozesse erfassen, auf denen menschliche Navigationsleistungen basieren (vgl. Aguirre et al., 1998; Aguirre \& D’Esposito 1999; Kindsmüller et al., 1999; Ku et al., 2003). Bei Betrachtung von Abbildung 1 wird klar, dass zur Bearbeitung derartiger zweidimensionaler Aufgaben nicht dieselben kognitiven Fähigkeiten notwendig sind wie zur räumlichen Orientierung im Alltag.

\footnotetext{
${ }^{11}$ Weitere Verfahren finden sich bei Blanchard et al., 1994; Heinrichs \& Zakzanis, 1998; Rund, 1998; Sanfilipo et al., 2002; Snitz et al., 2005 \& online unter www.hogrefe.de.
} 

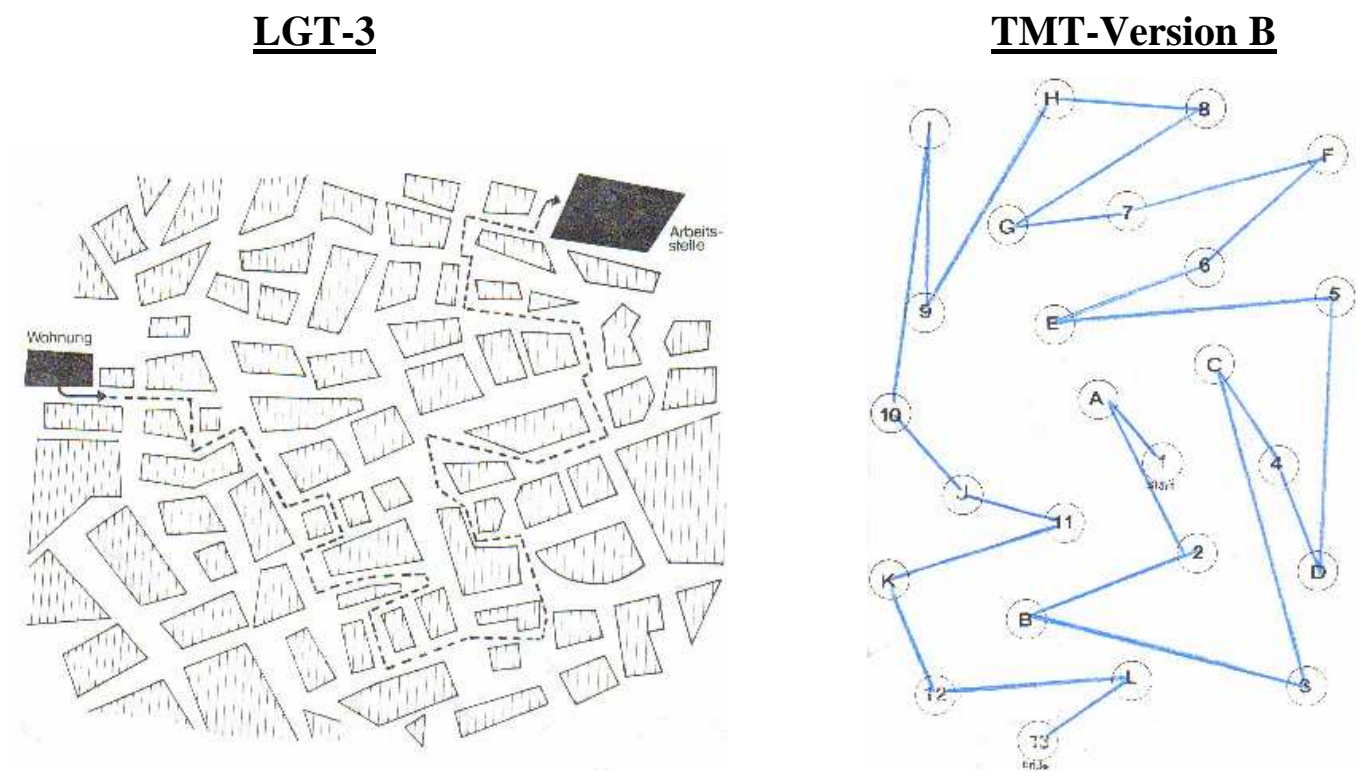

Abbildung 1: Beispiele für zweidimensionale „paper and pencil“ Tests zur Erfassung visuell-räumlicher Kognitionsleistungen

Visuell-räumliche Kognitionsprozesse wie die Navigation im extrapersonalen Raum können mit den traditionellen ,,paper and pencil” Tests also nur mit unzureichendem Realitäts- und Validitätsgrad erfasst werden: „...Standard tests...lack ecological validity since complex interactive and mostly self-generated behavior is not being assessed whereas real life is not at all structured and standardized... “ (van Beilen et al., 2005, S. 586; vgl. auch Lezak, 1995). Als Alternative etabliert sich zunehmend eine neue Technik, welche komplexe Navigationsprozesse mit maximaler Authenzität zu erfassen vermag: Das Paradigma der „Virtuellen Realität“ (vgl. Distler et al., 1998; Mehlitz et al., 1998; van Veen et al., 1998; Skelton et al., 2000; Mehlitz, 2004).

\subsubsection{Das Paradigma der, „Virtuellen Realität“"}

\subsection{Begriffsdefinition: Was ist die „Virtuelle Realität"?}

Unter dem Begriff Virtuelle Realität (VR, virtuell, engl. „virtual“ = scheinbar) werden heterogene, computergenerierte Versuchsdesigns mit unterschiedlicher technischer Realisierung zusammengefasst. Allen Virtuellen Realitäten ist dabei gemein, dass im Zentrum Mensch-Computer-Schnittstellen stehen, die es dem Benutzer erlauben, intuitiv und naturalistisch mit computergenerierten Umgebungen und Situationen zu interagieren und diese realitätsnah zu erleben (vgl. Pimentel und Teixeira, 1995; Darken et al., 1998; Carrozzo \& Lacquaniti, 1998; Mehlitz et al., 1998; Costa et al., 2002). Ziel virtueller Umwelten ist es also, realistische Simulationen von Prozessen, Objekten und Systemen zu erzeugen, mit denen die Testperson aktiv und mit maximaler ökologischer Validität 
interagieren kann (vgl. Welch et al., 1996; Aguirre \& D’Eposito, 1999). Abbildung 2 zeigt Beispiele computergenerierter dreidimensionaler Umwelten.
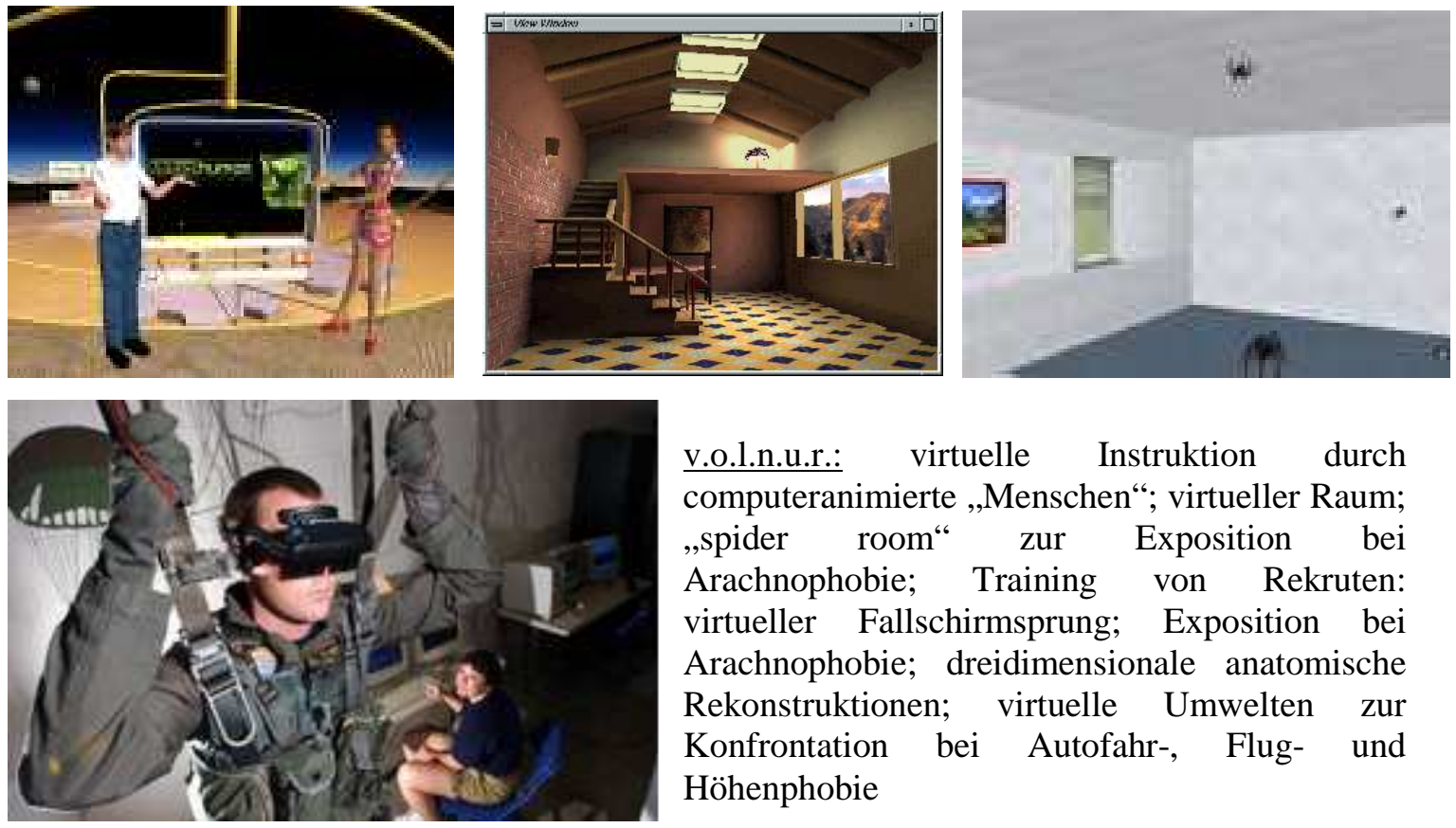

v.o.l.n.u.r.: virtuelle Instruktion durch computeranimierte „Menschen“; virtueller Raum; „spider room“ zur Exposition bei Arachnophobie; Training von Rekruten: virtueller Fallschirmsprung; Exposition bei Arachnophobie; dreidimensionale anatomische Rekonstruktionen; virtuelle Umwelten zur Konfrontation bei Autofahr-, Flug- und Höhenphobie
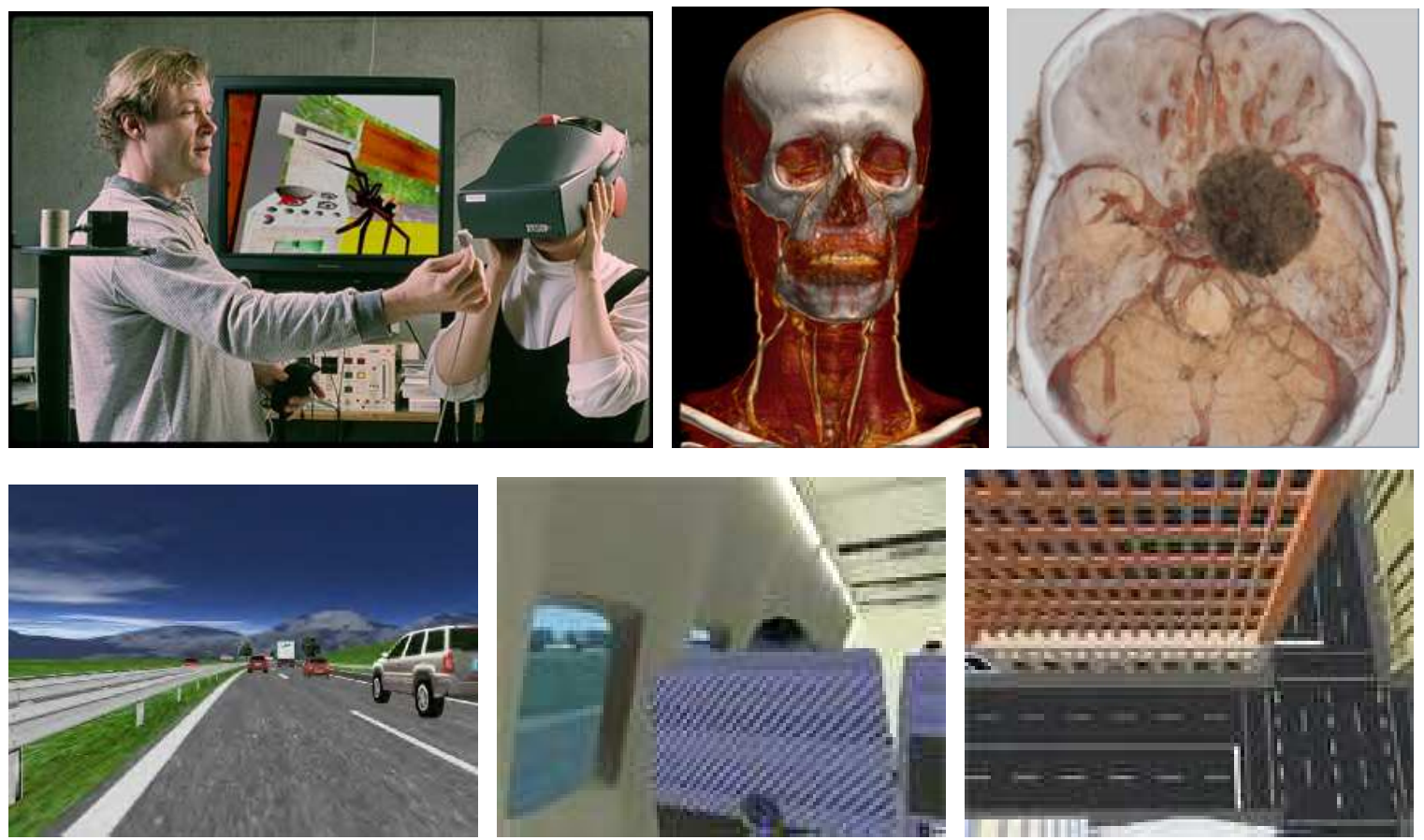

Abbildung 2: Beispiele für ,Virtuelle Realitäten“

Wie aus der Abbildung ersichtlich, werden virtuelle Umwelten bereits in den verschiedensten Anwendungsbereichen eingesetzt: Zum Beispiel im diagnostischen Setting zur Evaluation der Fahrtauglichkeit (Krüger et al., 2005), im Rahmen von Therapiesitzungen zur Konfrontationstherapie (z.B. Carlin et al., 1997; Glantz et al., 1997; Graap et al., 2004; Parrott et al., 2004; St-Jaques et al., 2004; Mühlberger et al., 2001, 
2005), zu Ausbildungszwecken - dem Training von Rekruten (Pausch et al., 1992; Witmer et al., 1996; Vargas, 2006) oder im Rahmen der Rehabilitation zur Wiederherstellung kognitiver Funktionen (Lampton et al., 1994; Kottlow et al., 2004). ${ }^{12}$

\subsection{Validitätsvorteile des VR-Paradigmas}

Dass sich virtuelle Testsysteme im klinischen Alltag und auch in der wissenschaftlichen Praxis zunehmend etablieren können, liegt insbesondere an den mit ihnen verbundenen Validitätsvorteilen:

Der Hauptvorteil virtueller Versuchsdesigns besteht in ihrer hohen ökologischen und prognostischen Validität, welche durch eine möglichst realistische Aufgabensituation erzielt wird (Buckwalter \& Rizzo, 1997). Virtuelle Realitäten sind durch

1) eine realitätsnahe Repräsentation der künstlichen Umgebung durch den Computer anhand visueller, eventuell zusätzlich auditiver oder taktiler Techniken,

2) eine Interaktion in Echtzeit (das heisst, zwischen dem Verhalten des Benutzers und der daraus folgenden Veränderung in der virtuellen Umgebung treten keine wahrnehmbaren Verzögerungen auf) und

3) eine Verwendung von am Menschen orientierten Interaktionsmöglichkeiten, die im Gegensatz zu Tastatur und Maus natürliche Bewegungen und Kommunikationswege wie Sprache und Gestik erlauben,

charakterisiert (Kaltenborn \& Rienhoff, 1993) und erlauben es so, multimodale Kognitionsleistungen mit hohem Realitätsgrad zu erfassen.

Eine Vielzahl an Evaluationsstudien konnte bereits zeigen, dass sich insbesondere visuellräumliche Navigationsleistungen so realitätsnah abbilden lassen, dass sie an die ökologische Validität von Feldversuchen heranreichen (Ruddle et al., 1997; van Veen et al., 1998; Waller et al., 1998; Costa et al., 2002). So können Orientierungs- und Navigationsleistungen in der virtuellen Testumgebung ebenso effizient trainiert werden wie im realen Setting (Waller et al., 1998; Ruddle et al., 1997, 1998). Zudem ist ein Transfer in die Realität beobachtbar (Rothbaum, 1995, 1996; Witmer et al., 1996; Carlin, 1997). Darüber hinaus korreliert die Leistung von Probanden in der Virtuellen Realität stark mit ihren alltäglichen Navigations- und Orientierungsproblemen sowie mit ihrer subjektiven Selbsteinschätzung (Skelton et al., 2000; Spiers et al., 2001). Durch diese Eigenschaften heben sich Virtuelle Realitäten klar von neuropsychologischen

\footnotetext{
${ }^{12}$ Ausführliche Informationen zu Konzeption, Einsatzgebieten und Evaluation der VR sind unter 7.5. zu finden; siehe zudem Pimentel \& Teixera (1995); Carrozzo \& Lancquaniti (1998) und Darken et al. (1998).
} 
Standardverfahren $\mathrm{ab}$ und besitzen „...für zahlreiche neuropsychologische Fragestellungen eine höhere Sensitivität und Testvalidität und damit einen besseren prädiktiven Wert für funktionelle Einschränkungen im Alltag “ (Mehlitz, 2004, S. 83; vgl. auch Pugnetti et al., 1998; Klinger et al., 2004). ${ }^{13}$

Neben ihrer hohen ökologischen, externen Validität zeichnen sich computergenerierte Versuchsdesigns zudem durch eine Vielzahl an methodischen Vorteilen und somit durch eine hohe interne Validität aus (für einen Überblick s. Distler et al., 1998; Mehlitz, 2004): So ist es beispielsweise möglich, Testumgebungen im Sinne der jeweiligen Fragestellungen zu konstruieren und dabei Test- und Moderatorvariablen - wie z.B. die Größe und Zahl der Wegmarken oder die Auftretenshäufigkeit von Distraktoren - flexibel $\mathrm{zu}$ variieren. In der vorliegenden Arbeit konnten so zwei Umwelten, das virtuelle Labyrinth und der virtuelle Park, zur Erfassung ego- und allozentrischer Navigationsprozesse konstruiert werden, die bis auf wenige Details identisch aufgebaut waren und den Wegesystemen anderer Autoren ähneln (vgl. Ruddle et al., 1997, 1998; Gillner \& Mallot, 1998; Waller et al., 1998).

Ein weiterer Vorteil besteht in der perfekten Kontrolle von Störfaktoren: Virtuelle Realitäten erlauben eine präzise Stimuluskontrolle, die die Modulation perzeptueller und motorischer Parameter auf einer feinen Timeskala erlaubt, eine hochstandardisierte, automatische Testadministration und die Erfassung mulipler Verhaltensvariablen mit hoher räumlicher und zeitlicher Auflösung bei Gewährleistung einer maximalen Erhebungs- und Auswertungsreliabilität, -validität sowie -objektivität (Carrozzo \& Lacquantini, 1998; Gillner \& Mallot, 1998; Waller et al., 1998; Ku et al., 2003; Hartley et al., 2003). Verschiedene Studiendesigns sind mit Hilfe Virtueller Realitäten folglich exakt kontrollierund somit auch replizierbar.

Ein großer Vorteil des VR-Paradigmas besteht des Weiteren in der Kombinierbarkeit mit bildgebenden Verfahren. Mit Hilfe Virtueller Realitäten ist es möglich, die aktive Navigationsleistung der Patienten zu erfassen, während sie gleichzeitig passiv, mit fixiertem Kopf im Scanner liegen, so dass simultan zur Erhebung der Neuropsychologie Variablen des Gehirns erfasst werden können (vgl. Aguirre \& D’Esposito 1997; Grön et al., 2000; Shelton \& Gabrieli; 2002, Hartley et al., 2003; Astur et al., 2004; Baumann, 2004; Lee et al., 2004).

\footnotetext{
${ }^{13}$ Für den interessierten Leser findet sich eine ausführliche Darstellung der Einsatzfelder der VR unter 7.5.1.4..
} 
Zusammengefasst ist es mit Hilfe des VR-Paradigmas also möglich, komplexe, multimodale Kognitionsleistungen in einem maximal authentischen Testsetting zu erfassen, das sich dem Realisimus von Feldversuchen annähert und gleichzeitig hohen methodischen Ansprüchen zu genügen, die denen von Laborstudien ähneln. Distler et al. (1998, S. 2) resümieren: „VEs provide researchers investigating human navigation behaviour with an experimental Setting which enables them to perform their studies in realistic environments under well controlled laboratory conditions“.

\subsection{Visuell-räumliche Navigationsprozesse}

Es gibt bereits eine Reihe von Studien, die visuell-räumliche Navigationsleistungen in der Virtuellen Realität untersuchten. Im Focus der meisten Studien stand allerdings nicht die Erfassung (defizitärer) neuropsychologischen Testleistungen, sondern vielmehr die technische Optimierung des virtuellen Paradigmas, um die Navigationsleistung gesunder Probanden, möglichst realitätsnah erfassen zu können (z.B. Witmer et al., 1996; Ruddle et al., 1997, 1998; Distler et al., 1998; Waller et al., 1998).

Viele dieser Befunde wurden bei der Konstruktion des vorliegenden Testsystems berücksichtigt, wie zum Beispiel die Auswahl eines Head Mounted Displays (HMD; vgl. 3.2.2.2.1.) als Anzeigesystem, welches im Gegensatz zu Präsentationen am PC zu einem höheren „Immersionsgefühl“ 14 und einem höheren „sense of presence“ führt und folglich die valideste Abbildung der visuell-räumlichen Orientierungsleistung erlaubt, oder die Auswahl von mehreren, fünfminütigen Trialzeiten, um einerseits durch relativ lange Explorationszeit die „familarity“, einen wichtigen Moderator der Navigationsleistung zu fördern (Waller et al., 1998; Ruddle et al., 1997, 1998), andererseits aber auch das Auftreten von Nebenwirkungen wie der ,simulator sickness“ zu vermeiden (vgl. 7.5.1.3.; siehe zudem Carlin et al., 1997). Darüber hinaus wurden in Anlehnung an Ruddle et al. (1997, 1998), Gillner \& Mallot (1998) und Waller et al., (1998) zwei unterschiedliche virtuelle Testumgebungen konzipiert: Das „virtuelle Labyrinth“ zur Erfassung egozentrischer Navigationsprozesse und der „virtuelle Park“ zur Erfassung allozentrischer Navigationsprozesse. Genauere Erläuterungen zu methodischen Aspekten finden sich unter 3.2.2. sowie bei Mehlitz (2004).

\footnotetext{
14 „Immersion“ (von engl.: to immerse = eintauchen, sich vertiefen in) erfasst, inwieweit sich der Benutzer in die virtuelle Umgebung hineinversetzt fühlt; , sense of presence “: mehrdimensionales Konstrukt; umfasst verschiedene subjektive Bewertungen der Testpersonen, wie das „Immersionsgefühl“, die Interaktionsfähigkeit mit der VR und die Höhe der auf das VR gerichteten Aufmerksamkeit. Das Konstrukt ist von zentraler Bedeutung, da es Einfluss auf die ökologische Validität der Testumgebung und somit auf die Navigationsleistung der Probanden nimmt (vgl. Sheridan, 1992; Slater et al., 1994; Hendrix \& Barfield, 1996; Welch et al., 1996; Slater \& Willbur, 1997; Mehlitz, 2004).
} 


\subsection{Das VR-Paradigma und schizophrene Testpersonen}

Das Paradigma der Virtuellen Realität bietet sich für die Untersuchung schizophrener Probanden besonders an, weil mit ihr synthetische, multimodale Kognitionsprozesse besser erfasst werden können als mit artifiziellen ,paper and pencil“" Tests - und weil insbesondere die Störung dieser höchsten, multimodalen Kognitionsleistungen als Ursache der schizophrenen Psychopathologie diskutiert wird (vgl. 1.1.4.3.1.2., zudem Peled, 1999; Costa et al., 2002; Ku et al., 2003). Folglich sollten sich bei der visuell-räumlichen Navigation noch größere Defizite abzeichnen als bei den ihr zugrunde liegenden neuropsychologischen Teilfunktionen, die in bisherigen Studien untersucht wurden (vgl. 1.1.4.3.3.).

Trotz ermutigender Ergebnisse gibt es bisher aber noch vergleichsweise wenige Studien, die das VR-Design mit Fragestellungen zur Schizophrenie kombinieren: Ku et al. (2003) und Astur et al. (2004) konnten mit Hilfe des Paradigmas bereits zeigen, dass schizophrene Probanden im Vergleich zu Kontrollpersonen Defizite in der Navigationsleistung aufzeigen. Kurtz et al. (2004) beobachteten darüber hinaus, dass schizophrene Probanden beim Autofahren weitaus langsamere Geschwindigkeiten erreichen als Vergleichspersonen und schlussfolgert, dass mit dem vorsichtigeren Fahren möglicherweise visuell-räumliche Defizite kompensiert werden.

Costa et al. (2002) focussierten in ihrer Arbeit auf die Möglichkeit der Rehabilitation schizophrener Patienten, indem sie sich bemühen, verschiedene kognitive Funktionen (wie Konzentration, Handlungsplanung, Orientierung) in einer realistischen ,virtuellen Stadt“ zu trainieren. Erste Ergebnisse geben Hinweise auf eine Verbesserung der Alltagsfähigkeit sowie auf eine Generalisierung wiedererlernter Fähigkeiten in den Alltag, wobei insbesondere die hohe intrinsische Motivation der Probanden beim Umgang mit den virtuellen Aufgaben eine große Rolle spielte.

Die bisherigen Studien deuten darauf hin, dass das Paradigma der Virtuellen Realität auch bei schizophrenen Patienten bedenkenlos eingesetzt werden kann; erste Befunde sprechen für Defizite in ihrer visuell-räumlichen Navigationsleistung. Auf dieser Grundlage zielt die vorliegende Arbeit darauf ab, ego- und allozentrische Navigationsprozesse schizophrener Patienten im multisensorischen, dreidimensionalen Raum zu erfassen und so eine der höheren, multimodalen Kognitionsleistungen zu untersuchen, die bei der schizophrenen Psychopathologie in besonderem Maße betroffen zu sein scheinen. 


\subsection{Zusammenfassung}

Der theoretische Überblick hat gezeigt, dass

1) eine Vielzahl an neuroanatomischen, psychopathologischen und neuropsychologischen Befunden auf eine Involvierung des Parietallappens bei der schizophrenen Psychopathologie hinweist, es bisher aber erst wenige systematische Studien gibt

2) es in der neuropsychologischen Schizophrenieforschung viele Hinweise auf Dysfunktionen in parietalen Funktionen - wie visuell-räumlichen Kognitionsleistungen - gibt, diese bisher aber vorwiegend mit klassischen, zweidimensionalen Testverfahren erfasst wurden, welche nur eine unzureichende Validität besitzen

3) das Paradigma der Virtuellen Realität die Möglichkeit bietet, visuell-räumliche Navigationsprozesse mit hoher interner und externer Validität zu erfassen

4) sich ego- und allozentrische Navigationsprozesse unterscheiden lassen, die von einem zugrunde liegenden parietohippocampalen Netzwerk realisiert werden

Folglich focussiert die vorliegende Studie erstmals darauf, Dysfunktionen in ego- und allozentrischen Navigationsleistungen bei schizophrenen Probanden mit Hilfe des VRParadigmas $\mathrm{zu}$ erfassen sowie eine Assoziation $\mathrm{zu}$ parietalen Cortexanomalien herzustellen. 


\section{Ableitung der Forschungshypothesen}

\subsection{Zentrale Forschungshypothesen}

\subsubsection{Hypothesen Neuropsychologie: Visuell-räumliche Navigationsleistung}

Bisher gibt es keine Studien $\mathrm{zu}$ ego- und allozentrischen Navigationsleistungen schizophrener Patienten. Aufgrund der Befunde zu defizitären visuell-räumlichen und propriozeptiven Kognitionsleistungen sowie auf Basis der Befunde von Ku et al. (2003) und Astur et al. (2004) ist allerdings davon auszugehen, dass auch die verschiedenen Navigationsleistungen bei der Schizophrenie beeinträchtigt sind. Es folgt:

\section{Hypothese 1:}

Schizophrene Probanden haben eine schlechtere egozentrische Navigation als gesunde Kontrollprobanden und erzielen daher in der virtuellen „Labyrinthaufgabe“ ein schwächeres Ergebnis als die gesunde Vergleichsgruppe.

Hypothese 2:

Schizophrene Probanden haben eine schlechtere allozentrische Navigation als gesunde Kontrollprobanden und erzielen daher in der virtuellen „Parkaufgabe“ ein schwächeres Ergebnis als die gesunde Vergleichsgruppe.

\subsubsection{Hypothesen Hirnvolumetrie: Parietale Cortexvolumina}

Aufgrund der neuroanatomischen Befundlage ist davon auszugehen, dass der Parietallappen an der neurobiologischen Genese der Schizophrenie wesentlich beteiligt ist und sich markante Volumenreduktionen beobachten lassen. Daraus ergibt sich:

\section{Hypothese 3:}

Schizophrene Testpersonen besitzen kleinere parietale Hirnvolumina als gesunde Kontrollprobanden.

\subsubsection{Zusammenhangshypothesen Neuropsychologie - Hirnvolumetrie}

Da davon ausgegangen werden kann, dass der Parietallappen ein wesentliches neuronales Korrelat visuell-räumlicher Navigationsprozesse darstellt, folgt des Weiteren 


\section{Hypothese 4:}

Defizite schizophrener Patienten in egozentrischen Navigationsprozessen korrelieren mit charakteristischen parietalen Volumenreduktionen: Je kleiner die parietalen Hirnvolumina, desto schlechter sind die Navigationsleistungen im egozentrischen Raum.

\section{Hypothese 5:}

Defizite schizophrener Patienten in allozentrischen Navigationsprozessen korrelieren mit charakteristischen parietalen Volumenreduktionen: Je kleiner die parietalen Hirnvolumina, desto schlechter sind die Navigationsleistungen im allozentrischen Raum.

\subsection{Explorative und validierende Hypothesen}

\subsubsection{Hypothesen zu hippocampalen Arealen}

Da Volumenreduktionen des medialen Temporallappens - und insbesondere hippocampaler Strukturen - bei der Schizophrenie häufig beobachtet wurden und da visuellräumlich Navigationsprozesse nach Meinung vieler Autoren (vgl. 1.2.1.2.3.) durch ein parieto-hippocampales Netzwerk repräsentiert werden, ergeben sich explorativ folgende Hypothesen:

Hypothese 6:

Schizophrene Testpersonen besitzen kleinere hippocampale Volumina als gesunde Kontrollprobanden.

\section{Hypothese 7:}

Defizite schizophrener Patienten in ego- und allozentrischen Navigationsprozessen korrelieren mit hippocampalen Volumenreduktionen: Je kleiner die hippocampalen Hirnvolumina, desto schlechter sind die Navigationsleistungen im ego- und allozentrischen Raum.

\subsubsection{Hypothesen zu schizophrenen Subgruppen}

Wie bereits unter 1.1.2. erwähnt, gibt es zunehmende Bemühungen, die schizophrenen Subgruppen auch durch differentielle neuropsychologische und neuroanatomische Profile zu charakterisieren. Ob sich subtypenspezifische Effekte zeigen, wird in der vorliegenden Arbeit mit folgenden explorativen Hypothesen untersucht: 


\section{Hypothese 8:}

Die schizophrenen Subgruppen unterscheiden sich in ihrer visuell-räumlichen Navigationsleistung: Es finden sich Unterschiede im egozentrischen sowie im allozentrischen Raum.

\section{Hypothese 9:}

Die schizophrenen Subgruppen unterscheiden sich in der Größe ihrer parietalen Hirnvolumina.

\subsubsection{Hypothesen zu klassischen neuropsychologischen Verfahren}

Um die vorliegenden Testergebnisse mit denen neuropsychologischer Standardinstrumente vergleichen und anhand dieser Befunde valdieren zu können, wurden die Wechsler Memory Scale-Revised (WMS-R) sowie der Trail Making Test A und B (TMT A und B) erhoben. Entsprechend der neuropsychologischen Literatur (vgl. 1.1.3.) ist von folgenden Hypothesen auszugehen:

\section{Hypothese 10}

Die Gedächtnisleistungen schizophrener Probanden sind defizitär: Sie weisen in der WMS$\mathrm{R}$ schwächere Leistungen auf als gesunde Kontrollpersonen.

\section{Hypothese 11}

Die visuomotorische Aktionsgeschwindigkeit schizophrener Probanden ist defizitär: Sie weisen im TMT A und B schwächere Leistungen auf als gesunde Kontrollpersonen. 


\section{Methoden}

\subsection{Stichprobe}

\subsubsection{Stichprobenumfang}

In der aktuellen Studie wurden 78 Probanden untersucht. Bei der Hälfte der Testpersonen handelte es sich um Patienten, die sich aufgrund einer akuten psychotischen Episode im Universitätsklinikum Göttingen behandeln ließen, bei der anderen Hälfte der Population handelte es sich um gesunde Kontrollpersonen. Eine Stichprobengröße von rund 30 Probanden pro Gruppe leitete sich aus vergleichbaren Arbeiten ab, die mit Probandenzahlen zwischen zehn und 30 Testpersonen aussagekräftige Ergebnisse zu vergleichbaren Fragestellungen erzielen konnten (z.B. Daprati et al., 1997; Niznikiewicz et al., 2000; Ku et al., 2003; Astur et al., 2004, vgl. auch McCarley et al., 1999; Shenton et al., 2001). ${ }^{15}$

\subsubsection{Rekrutierung der Versuchspersonen}

Die Probanden der schizophrenen Versuchsgruppe wurden aus der psychiatrischen Abteilung des Universitätsklinikums Göttingen rekrutiert. Die Rekrutierung erfolgte durch die behandelnden Ärzte oder Psychologen der jeweiligen Station, sofern sie den Aufnahmekriterien der Studie entsprachen. Die Diagnose „Schizophrenie“ sowie deren Typisierung in die verschiedenen Subgruppen (paranoid vs. desorganisiert vs. undifferenziert) erfolgte anhand des Strukturierten Klinischen Interviews für DSM-IV (SKID; Wittchen et al., 1997).

Testpersonen der gesunden Kontrollgruppe wurden über Aushänge in öffentlichen Einrichtungen geworben. Für ihre Teilnahme erhielten sie eine Aufwandsentschädigung von 25 Euro sowie eine Fahrtkostenrückerstattung, falls diese gewünscht wurde. Zudem wurden sie über die Zielstellung der Studie sowie über ihre persönlichen Testleistungen informiert.

\subsubsection{Charakterisierung der Stichprobe}

\subsubsection{Ein- und -Ausschlusskriterien}

Sowohl die Probanden der Patientengruppe, als auch die Probanden der Kontrollgruppe mussten verschiedene Ein- und Ausschlusskriterien erfüllen, um in die Studie aufgenommen zu werden:

\footnotetext{
${ }^{15}$ Anzumerken ist, dass sich hinter den verschiedenen Testteilen (,Virtuelle Realität“ vs. Hirnanatomie vs. klassische Neuropsychologie) zum Teil unterschiedliche Stichprobengrößen verbergen. Ursache hierfür ist, dass nicht alle Patienten in der Lage waren, alle Versuchselemente adäquat zu bewältigen. Als Kontrollgruppe wurde jeweils eine entsprechende Subpopulation der gesunden Probandengruppe ausgewählt.
} 
Bei der schizophrenen Probandengruppe handelte es sich um Patienten mit einer akuten floriden Symptomatik, die sich zum Untersuchungszeitpunkt in einem klinisch stabilen Zustand befinden mussten. Keine Testperson durfte - abgesehen von der psychotischen Erkrankung der Patientengruppe - eine (komorbide) psychiatrische, internistische, neurologische oder hirnorganische Erkrankung aufweisen. Zudem sollten alle Versuchspersonen zwischen 16 und 60 Jahren alt sein und einen Intelligenzquotienten von mindestens 80 Punkten besitzen. Da bei Probanden mit höherem Lebensalter oder geringerem IQ kognitive Defizite unabhängig von einer Psychopathologie zu erwarten wären, wurden diese von der Studienteilnahme ausgeschlossen.

Die Ein- und Ausschlusskriterien ähneln denen vergleichbarer Arbeiten zu neuropsychologischen Testleistungen und Hirnmorphologie schizophrener Patienten (z.B. Andreasen, 1990; Wilke et al., 2001; Pol et al., 2001; Keshavan et al., 2003; Badcock et al., 2005).

\subsubsection{Vergleich der Probandengruppen - Soziodemographische Daten}

Zur Minimierung von Fehlervarianz wurden eine Patienten- und eine Kontrollgruppe getestet, die sich in wichtigen Stichprobencharakteristika ähnelten. Tabelle 4 gibt einen Überblick:

Tabelle 4: $\quad$ Stichprobencharakteristika der Patienten- \& der Kontrollgruppe

\begin{tabular}{|c|c|c|c|c|c|c|}
\hline \begin{tabular}{|l} 
Gruppe: \\
Stichprobenvariable:
\end{tabular} & \multicolumn{2}{|c|}{$\frac{\underline{\text { Schizophrene }}}{\text { Patientengruppe }}$} & \multicolumn{2}{|c|}{$\frac{\begin{array}{c}\text { Gesunde } \\
\text { Kontrollgruppe }\end{array}}{(n=39)}$} & \multicolumn{2}{|c|}{$\frac{\text { Testverfahren und }}{\underline{\text { Prüfgröße }^{\#}}}$} \\
\hline Geschlecht & $\underline{\text { Häufigkeit }}$ & Prozent & Häufigkeit & $\underline{\text { Prozent }}$ & & \\
\hline 1) weiblich & 15 & $38,5 \%$ & 15 & $38,5 \%$ & & Nicht \\
\hline 2) männlich & 24 & $61,5 \%$ & 24 & $61,5 \%$ & & signifikant \\
\hline \multirow[t]{2}{*}{$\underline{\text { Alter }}$} & $\underline{\mathrm{MW}}$ & $\underline{\mathrm{s}}$ & $\underline{\mathrm{MW}}$ & $\underline{\mathrm{S}}$ & $\underline{\mathrm{t}-\text { Wert }}$ & $\underline{\text { Signifikanz }}$ \\
\hline & 29,85 Jahre & 9,13 & 33,21 Jahre & 9,90 & 1,560 & $\mathrm{p}=.123$ \\
\hline Händigkeit & $\underline{\text { Häufigkeit }}$ & $\underline{\text { Prozent }}$ & $\underline{\text { Häufigkeit }}$ & $\underline{\text { Prozent }}$ & $\chi^{2}$-Wert & Signifikanz \\
\hline 1) rechts & 35 & $89,7 \%$ & 34 & $87,2 \%$ & 1,514 & $\mathrm{p}=.469$ \\
\hline 2) links & 3 & $7,7 \%$ & 5 & $12,8 \%$ & & \\
\hline 3) ambidexter & 1 & $2,6 \%$ & 0 & $0 \%$ & & \\
\hline
\end{tabular}

MW = Mittelwert; s = Standardabweichung; ${ }^{\#}$ Testverfahren und Prüfgröße = T-Test, t-Wert und ChiQuadrat-Test, $\chi^{2}$-Wert. 
Tabelle 4: $\quad$ Stichprobencharakteristika der Patienten- \& der Kontrollgruppe (Forts.)

\begin{tabular}{|c|c|c|c|c|c|c|}
\hline $\begin{array}{r}\text { Gruppe: } \\
\text { Stichprobenvariable: }\end{array}$ & \multicolumn{2}{|c|}{$\frac{\frac{\text { Schizophrene }}{\text { Patientengruppe }}}{(n=39)}$} & \multicolumn{2}{|c|}{$\frac{\begin{array}{c}\text { Gesunde } \\
\text { Kontrollgruppe }\end{array}}{(n=39)}$} & \multicolumn{2}{|c|}{$\frac{\text { Testverfahren und }}{\underline{\text { Prüfgröße }^{\#}}}$} \\
\hline$\frac{\text { Intelligenzquotient }}{\text { (HAWIE-R) }}$ & $\frac{\mathrm{MW}}{112,87}$ & $\begin{array}{c}\underline{\mathrm{s}} \\
19,40\end{array}$ & $\frac{\mathrm{MW}}{118,90}$ & $\begin{array}{c}\underline{\mathrm{s}} \\
16,13\end{array}$ & $\frac{\mathrm{t}-\mathrm{Wert}}{1,492}$ & $\begin{array}{c}\frac{\text { Signifikanz }}{\mathrm{p}=.140} \\
\mathrm{p}\end{array}$ \\
\hline $\begin{array}{l}\text { Schulbildung } \\
\text { 1) } \text { Hauptschule } \\
\text { 2) Realschule } \\
\text { 3) Gymnasium }\end{array}$ & $\begin{array}{c}\text { Häufigkeit } \\
7 \\
9 \\
23\end{array}$ & $\begin{array}{l}\text { Prozent } \\
17,9 \% \\
23,1 \% \\
59,0 \%\end{array}$ & $\begin{array}{c}\text { Häufigkeit } \\
10 \\
15 \\
14\end{array}$ & $\begin{array}{l}\underline{\text { Prozent }} \\
25,6 \% \\
38,5 \% \\
35,9 \%\end{array}$ & $\frac{\chi 2 \text {-Wert }}{4,219}$ & $\frac{\text { Signifikanz }}{\mathrm{p}=.121}$ \\
\hline Ausbildungsdauer & $\frac{\mathrm{MW}}{14,26}$ & $\begin{array}{c}\underline{\mathrm{s}} \\
4,02\end{array}$ & $\frac{\text { MW }}{13,91}$ & $\begin{array}{c}\underline{\mathbf{s}} \\
3,71\end{array}$ & $\frac{\mathrm{t} \text {-Wert }}{-0,396}$ & $\frac{\text { Signifikanz }}{\mathrm{p}=.694}$ \\
\hline
\end{tabular}

MW = Mittelwert; $s=$ Standardabweichung; ${ }^{\#}$ Testverfahren und Prüfgröße $=$ T-Test, $t$-Wert und ChiQuadrat-Test, $\chi^{2}$-Wert.

Wie aus der Tabelle ersichtlich, setzte sich sowohl die Patienten-, als auch die Kontrollgruppe aus 15 weiblichen und 24 männlichen Versuchsteilnehmern zusammen, die im Durchschnitt 29,85 beziehungsweise 33,21 Jahre alt waren. Die gesunde Probandengruppe setzte sich aus 34 Rechts- und fünf Linkshändern zusammen. Dem standen 35 rechtshändige, drei linkshändige und eine beidhändige Versuchsperson in der schizophrenen Patientengruppe gegenüber.

Der durchschnittliche Intelligenzquotient betrug 112,87 in der Patientengruppe, die sich aus sieben Hauptschülern, neun Realschülern und 23 Gymnasiasten zusammensetzte. In der Kontrollgruppe befanden sich zehn Hauptschüler, 15 Realschüler, und 14 Gymnasiasten, die im Mittel 118,90 IQ-Punkte besaßen. Probanden der Patienten- sowie der Kontrollgruppe konnten mit durchschnittlich 14,26 und 13,91 Jahren eine ähnlich lange Dauer in ihrer Berufsausbildung aufweisen.

In den untersuchten Populationen ließen sich keine signifikanten Unterschiede im Hinblick auf Geschlecht (jeweils 15 Frauen und 24 Männer), Alter $(t=1,560, p=.123)$, Händigkeit $\left(\chi_{2}=1,514, \mathrm{p}=.469\right)$, Intelligenz $(\mathrm{t}=1,492, \mathrm{p}=.140)$, Schulbildung $\left(\chi_{2}=4,219, \mathrm{p}=.121\right)$ oder Ausbildungsdauer $(\mathrm{t}=-0,396, \mathrm{p}=.694)$ feststellen. Anzumerken ist, dass die Patientengruppe ein überdurchschnittliches Intelligenz- und Edukationsniveau in Relation zu ihrer Grundpopulation aufweist. Auf mögliche Implikationen für die Interpretation der Versuchsergebnisse wird unter 5.4. eingegangen. 


\subsubsection{Charakterisierung der schizophrenen Probandengruppe}

\subsection{Ergänzende Soziodemographie}

Ergänzend zur bisher geschilderten Soziodemographie, scheinen im Hinblick auf die schizophrenen Probanden zudem die in Tabelle 5 aufgeführten Kennwerte relevant:

Tabelle 5: $\quad$ Soziodemographische Kennwerte der schizophrenen Probanden

\begin{tabular}{|c|c|c|c|c|c|}
\hline \multicolumn{6}{|c|}{ Schizophrene Probandengruppe $(n=39)$} \\
\hline $\begin{array}{l}\text { Erstmanifestation } \\
\text { 1) ja } \\
\text { 2) nein }\end{array}$ & $\begin{array}{c}\text { Häufigkeit } \\
15 \\
24\end{array}$ & $\begin{array}{l}\text { Prozent } \\
38,5 \% \\
61,5 \%\end{array}$ & $\begin{array}{c}\text { Alter der } \\
\text { Erstmanifestation }\end{array}$ & $\frac{\text { MW }}{26,03 \text { Jahre }}$ & $\begin{array}{c}\underline{\mathrm{s}} \\
8,01\end{array}$ \\
\hline Erkrankungsdauer & $\frac{\mathrm{MW}}{3,85 \text { Jahre }}$ & $\begin{array}{c}\underline{\mathrm{S}} \\
5,98\end{array}$ & $\begin{array}{l}\text { Anzahl vorheriger } \\
\text { Hospitalisierungen }\end{array}$ & $\frac{\mathrm{MW}}{1,85}$ & $\begin{array}{c}\underline{\mathrm{s}} \\
2,77\end{array}$ \\
\hline $\begin{array}{l}\text { Antipsychotische } \\
\text { Medikation } \\
\text { 1) Klass.Neuroleptika } \\
\text { 2) Atyp. Neuroleptika }\end{array}$ & $\begin{array}{c}\text { Häufigkeit } \\
9 \\
30\end{array}$ & $\begin{array}{l}\text { Prozent } \\
23,1 \% \\
76,9 \%\end{array}$ & $\begin{array}{l}\text { Akinetongabe? } \\
\text { 1) Ja } \\
\text { 2) Nein }\end{array}$ & \begin{tabular}{|c} 
Häufigkeit \\
6 \\
33
\end{tabular} & $\begin{array}{l}\text { Prozent } \\
15,4 \% \\
84,6 \%\end{array}$ \\
\hline
\end{tabular}

MW = Mittelwert; $\mathbf{s}=$ Standardabweichung.

Bei 15 der 39 Patienten handelte es sich zum Testzeitpunkt um eine Erstmanifestation der schizophrenen Erkrankung, 24 Probanden hatten sich hingegen mindestens ein Mal zuvor aufgrund ihrer Psychose in stationäre Behandlung begeben. Die Patienten befanden sich zuvor durchschnittlich 1,85 Mal in psychiatrischer Betreuung und waren im Durchschnitt seit 3,85 Jahren erkrankt. Das Alter der Erstmanifestation lag im Mittel bei 26,03 Jahren.

Zum Testzeitpunkt wurden neun Probanden ausschließlich mit klassischen Neuroleptika mediziert, während 30 Patienten atypische Neuroleptika erhielten. Sechs schizophrene Testpersonen wurden ergänzend mit Akineton behandelt. Inwiefern dies einen Einfluss auf die Versuchsergebnisse haben könnte, wird unter 5.4. diskutiert.

\subsection{Klinische Erhebungsverfahren}

Zur differenzierten Erfassung der Psychopathologie dienten verschiedene Fremdbeurteilungsverfahren, die bereits in einer Vielzahl vergleichbarer Arbeiten zum Einsatz kamen (vgl. Turetsky et al., 1995; Watzl \& Rist, 1997; Thompson et al., 2001; Wilke et al., 2001; Sanfilipo et al., 2002; Roob, 2002; Exner et al., 2004; Weniger et al., 2004a,b). 


\subsection{SKID}

Wie bereits erwähnt, erfolgte die Diagnosestellung anhand des Strukturierten Klinischen Interviews nach DSM-IV (Wittchen et al., 1997). Bei dem SKID handelt es sich um ein bewährtes, semistrukturiertes klinisches Interview, das dem Interviewer eine schnelle, reliable und valide Diagnose und Differentialdiagnose nach DSM-IV (APA, 1994) erlaubt. Das SKID besitzt eine bessere Interrater- und Retest-Reliabilität als alternative Checklisten oder halbstrukturierte Interviews und ist das derzeit überlegene Verfahren (vgl. Watzl \& Rist, 1997). Für eine detaillierte Darstellung siehe Brähler et al. (2002b).

\subsection{GAF}

Das Global Assessment of Functioning (Wittchen et al., 1997) ist ein kurzes, ökonomisches Verfahren, das in der klinischen Praxis häufig eingesetzt wird. Es schätzt die globale Leistungsfähigkeit der Patienten im Hinblick auf die psychische, soziale und berufliche Situation auf einem hypothetischen Kontinuum von „0“ entsprechend „seelischer Krankheit“ bis „100“, entsprechend ,seelischer Gesundheit“ ein.

\subsection{CGI}

Die Skala Clinical Global Impressions (AMDP und CIPS, 1990) wurde im Rahmen von Schizophreniestudien entwickelt. Mit ihr wird der „Schweregrad der Erkrankung“ auf einer Skala von 0 bis 7 beurteilt, wobei als Referenzgruppe die Grundpopulation der jeweiligen Psychopathologie (hier: Schizophrenie) herangezogen wird. Obwohl für die CGI keine Daten zu Normierung und keine Testgütekriterien vorliegen, wird sie auf ihrer hohen Augenscheinvalidität und Ökonomie häufig - so auch in der vorliegenden Studie eingesetzt.

\subsection{BPRS}

Die Brief Psychiatric Rating Scale (Overall \& Gorham, 1962) erfasst 18 psychiatrische Symptomkonstrukte auf einer jeweils 7-stufigen Skala, wobei „1“ mit „,nicht vorhanden“ gleichzusetzen ist und „7“ einer ,extrem stark“ ausgeprägten Symptomatik entspricht. Das Rating erfolgt anhand eines semistandardisierten Interviews, die Auswertung über die Bildung des Mittelwerts, der den Schweregrad der psychiatrischen Erkrankung wiedergibt. Die BPRS zeichnet sich bei Reliabilitäten zwischen $r=.87$ und $r=.97$ und einer Kriteriumsvalidität von $r=.93$ durch sehr gute Testgütekriterien aus und eignet sich neben der initialen Diagnostik auch zur Verlaufsmessung psychiatrischer Krankheitsbilder. 
Im Hinblick auf die explorative Fragestellung nach subgruppenspezifischen neuropsychologischen und hirnanatomischen Profilen, erweist es sich als besonders vorteilhaft, dass die BPRS eine Bildung der drei Subskalen - „psychotische Symptome“, „Rückzug/Retardierung“ und „Desorganisation“ - erlaubt, die es ermöglichen sollten, zwischen den drei postulierten schizophrenen Subpopulationen zu differenzieren (vgl. Liddle, 1987; Höschel et al., 1998). Die Skala „psychotische Symptome“ setzt sich dabei aus den Items „Wahn“ und „Halluzinationen“ zusammen, die Skala „Rückzug/Retardierung“ aus „emotionale Zurückgezogenheit“, „,affektive Abstumpfung“ und „motorische Verlangsamung“, während die Skala „Desorganisation“ aus den EinzelItems „Zerfall der Denkprozesse“ und „Maniriertheit“ aufgebaut ist.

\subsection{SAPS und SANS}

Ergänzend zur BPRS wurden die „Skala für die Beurteilung positiver Symptome“ (Andreasen, 1984) sowie die „Skala für die Beurteilung negativer Symptome“ (Andreasen, 1983) als reliable und valide Fremdeinschätzungsverfahren erhoben; Verfahren, die in der klinischen Praxis und Forschung stark etabliert sind (vgl. Peralta et al., 1994; Watzl \& Rist, 1997).

Die SAPS besteht aus 30 Items, die verschiedene Komponenten der psychotischen Positivsymptomatik erfassen, zudem aus fünf Items zur Globalbeurteilung der Dimensionen Halluzinationen, Wahnphänomene, bizarres Verhalten, positive formale Denkstörungen sowie unangemessener Affekt. Jedes der insgesamt 35 Items wird auf einer 5-stufigen Skala eingeschätzt, wobei der Wert „,0“ „nicht vorhanden“ bedeutet und der Wert ,5“ einer starken Symptomintensität entspricht.

Die SANS setzt sich aus 19 Items zur Beurteilung der Negativsymptomatik zusammen sowie aus fünf Items zur globalen Beurteilung der zugrunde liegenden Symptomdimensionen Affektive Verflachung, Alogie, Apathie/Willensschwäche, Anhedonie/Ungeselligkeit und Aufmerksamkeit. Analog zur SAPS wird auch bei der SAPS anhand einer 5-stufigen Skala geratet.

Die Auswertung beider Skalen erfolgt über die Berechnung von Mittelwerten. Von besonderer Relevanz ist auch hier die Möglichkeit zur Bildung von Symptomdimensionen (vgl. Liddle, 1987; Höschel, 1998). Ähnlich wie bei der BPRS besteht die Skala Positivsymptomatik aus den Items „Halluzinationen“ und „Wahnphänomene“, die Skala Negativsymptomatik aus den Items „Affektive Verflachung“, „Alogie“, „Apathie/Willensschwäche“ und „Anhedonie/Ungeselligkeit“ und die Skala 
„Desorganisation“ aus „Bizarres Verhalten“, „positive formale Denkstörungen“, „unangemessener Affekt“" und „Aufmerksamkeit“.

\subsection{Psychopathologische Kennwerte}

Tabelle 6 gibt einen Überblick über die Psychopathologie der schizophrenen Probanden:

Tabelle 6: $\quad$ Psychopathologische Kennwerte der schizophrenen Probanden

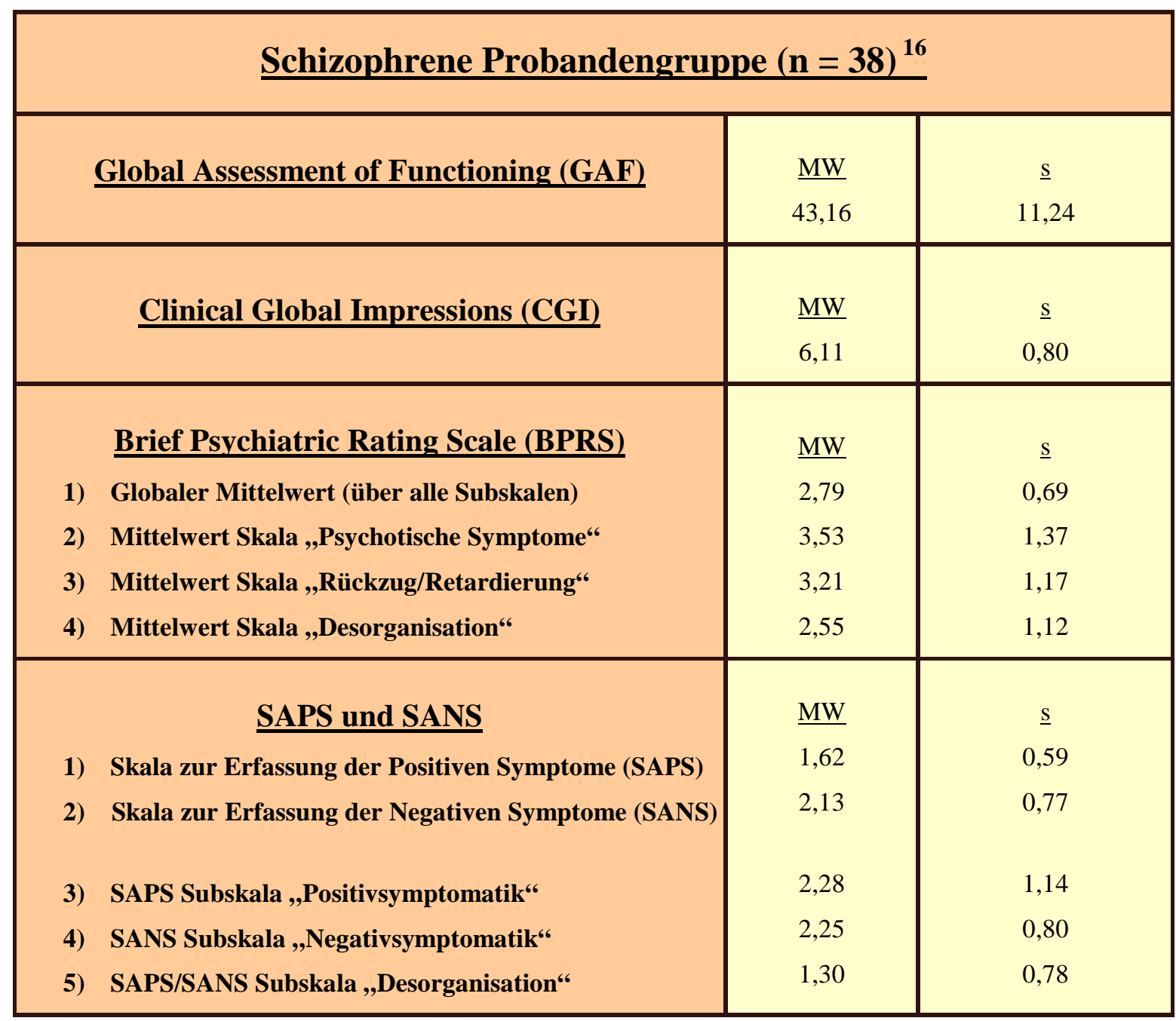

MW = Mittelwert; $\mathbf{s}=$ Standardabweichung.

Wie aus der Darstellung ersichtlich, handelte es sich bei der aktuellen Patientenstichprobe um eine stark eingeschränkte Probandenpopulation (CGI: MW = 6,11) mit nur geringem Funktionsniveau (GAF: MW =43,16). Die Psychopathologie war moderat ausgeprägt und äußerte sich vor allem in psychotischen, positiven (BPRS: MW = 3,53; SAPS: MW = 2,28) sowie in regressiven, negativen Symptomen (BPRS: MW = 3,21; SANS: $\mathrm{MW}=2,25$ ), während sich auf der Symptomdimension „Desorganisation“ nur leichte Effekte zeigten (BPRS: $\mathrm{MW}=2,55 ;$ SAPS/SANS: MW = 1,30).

\footnotetext{
${ }^{16}$ Die Psychopathologie eines Patienten fehlt, da dieser zum Erhebungszeitpunkt bereits entlassen war.
} 


\subsection{Schizophrene Subgruppen}

Um innerhalb der explorativen Analyse das Vorliegen differentieller neuropsychologischer und hirnanatomischer Profile innerhalb verschiedener schizophrener Subtypen prüfen zu können, wurden die Patienten mit Hilfe des SKID (Wittchen et al., 1997) den Subgruppen „paranoid“, „desorganisiert“ und „undifferenziert“ zugeordnet. Tabelle 7 zeigt die Zusammensetzung in der vorliegenden Patientenpopulation.

Tabelle 7: $\quad$ Psychopathologische Kennwerte der schizophrenen Subgruppen

\begin{tabular}{|c|c|c|c|c|c|}
\hline & \multicolumn{5}{|c|}{ Schizophrene Subgruppen $(\mathrm{n}=39)$} \\
\hline \multirow[t]{2}{*}{ Subtypen Schizophrenie } & Häufigkeit & Prozent & Positivsymptomatik & Negativsymptomatik & Desorg. Symptome \\
\hline & & & $\underline{\mathrm{MW}(\mathrm{s})^{17}}$ & $\underline{\mathrm{MW}(\mathrm{s})}$ & $\underline{\mathrm{MW}(\mathrm{s})}$ \\
\hline 1) paranoid & 23 & $59,0 \%$ & $2,55(1,21)$ & $2,11(0,67)$ & $1,14(0,53)$ \\
\hline 2) desorganisiert & 7 & $17,9 \%$ & $1,57(0,93)$ & $2,75(0,41)$ & $2,04(1,29)$ \\
\hline 3) undifferenziert & 7 & $17,9 \%$ & $2,36(0,95)$ & $2,46(1,04)$ & $1,25(0,38)$ \\
\hline 4) schizoaffektiv & 2 & $5,1 \%$ & $1,50(0,00)$ & $1,25(1,41)$ & $0,75(0,70)$ \\
\hline
\end{tabular}

MW = Mittelwert; s = Standardabweichung.

Die Patientengruppe setzte sich aus 23 Patienten mit paranoidem, sieben Patienten mit desorganisiertem und sieben Patienten mit undifferenziertem Schrizophenietypus zusammen. Darüber hinaus umfasste die Gruppe zwei Patienten mit der Diagnose einer „schizoaffektiven Psychose“. Der katatone sowie der residuale Subtyp waren in der vorliegenden Stichprobe nicht vertreten.

Die „paranoid-halluzinatorische Psychose“ war mit 23 Probanden der am häufigsten auftretende Subtypus. Patienten dieser Kategorie wiesen vor allem Symptome einer Positivsymptomatik, wie Wahnvorstellungen und Halluzinationen auf (vgl. Tabelle 7). Im Vergleich zu desorganisierten Patienten litten paranoid-schizophrene Testpersonen deutlich häufiger unter diesen Symptomen.

Sieben Patienten gehörten dem desorganisierten Typus der Schizophrenie an. Charakteristisch für diese sind vor allem bizarre Verhaltensweisen, formale Denkstörungen, defizitäre Aufmerksamkeitsprozesse sowie das Auftreten von Parathymien, also dem Kontext unangemessenen affektiven Reaktionen. Darüber hinaus

\footnotetext{
${ }^{17}$ Die vorliegenden Subskalen beruhen auf der SAPS und der SANS. Sie entsprechen im Wesentlichen den Subskalen der BPRS; vgl. auch 3.1.3.3.3..
} 
treten häufig Symptome einer Negativsymptomatik auf, wie affektive Verflachung, Alogie, Anhedonie und Apathie.

Entsprechend konnten in der desorganisierten Gruppe die höchsten Werte im Hinblick auf die negative sowie auf die desorganisierte Symptomdimension beobachtet werden.

Die sieben Probanden der undifferenzierten Subgruppe nahmen bezüglich der drei aufgeführten Skalen eine Zwischenstellung ein. Sowohl bezüglich Positiv- und Negativsymptomatik, als auch im Hinblick auf desorganisierte Symptome befanden sich ihre Punktwerte zwischen den beiden anderen Subgruppen: Wie zu erwarten, wiesen sie weniger psychotische Symptome auf als die paranoide Gruppe, aber mehr als die desorganisierte Gruppe. Negative und desorganisierte Symptome waren deutlicher zu beobachten als bei der paranoiden Gruppe, aber weniger stark ausgeprägt als in der desorganisierten Gruppe.

Insgesamt decken sich die beobachteten, subgruppenspezifischen Symptomkonstellationen mit der ihr zugrunde liegenden Klassifikation: Paranoid-Schizophrene zeigten vor allem paranoide/psychotische Symptome, Desorganisiert-Schizophrene waren primär durch desorganisierte und negative Merkmale charakterisiert und Undifferenziert-Schizophrene wiesen eine Mischung all dieser Symptome auf, allerdings in weniger eindrücklichem Ausprägungsgrad. Die Fragebogendaten deckten sich folglich mit den diagnostischen Schizophrenie-Subtypen und validieren so die mit dem SKID (Wittchen et al., 1997) vorgenommene Klassifikation. 


\subsection{Variation der unabhängigen Variablen}

\subsection{1. Überblick}

Der vorliegenden Studie lagen zwei quasiexperimentelle und eine experimentelle Versuchsvariable zugrunde: Der Faktor „Gruppenzugehörigkeit“ (UV1) wurde in zwei Stufen variiert - Testleistungen und Hirnanatomie von gesunden Kontrollpersonen wurden mit denen von schizophrenen Patienten verglichen. Des Weiteren wurde die Patientengruppe in drei Subgruppen aufgesplittet (UV2): Der Vergleich des paranoiden, des desorganisierten und des undifferenzierten Schizophrenietypus mit der gesunden Kontrollgruppe im Hinblick auf die abhängigen Variablen stellte den zweiten Faktor der vorliegenden Arbeit dar.

Darüber hinaus wurden mit dem ,,virtuellen Labyrinth“ und dem „,virtuellen Park“ zwei unterschiedliche Testumgebungen präsentiert (UV3), um die visuell-räumliche Navigationsleistung im egozentrischen und allozentrischen Raum erfassen zu können. Abbildung 3 gibt einen Überblick.

UV1: $\quad$ Gruppe (Schizophrene Probandengruppe vs. gesunde Kontrollgruppe)

UV2: $\quad$ Subgruppe (paranoid vs. desorganisiert vs. undifferenziert vs. gesund)

UV3: Virtuelle Testumgebung (Labyrinth vs. Park)

Abbildung 3: Unabhängige Variablen

Da soziodemographische und psychopathologische Kennwerte der verschiedenen Testgruppen, also die Variation des ersten und zweiten Faktors, bereits unter 3.1.3. erläutert wurden, soll an dieser Stelle auf eine weitere Differenzierung verzichtet werden. Es folgt die Darstellung der beiden virtuellen Testumgebungen „VR-Labyrinth“ und „,VRPark“.

\subsubsection{Die „Virtuellen Realitäten“"}

Zur Erfassung der visuell-räumlichen Navigationsleistung kam ein virtuelles Testsystem zum Einsatz, welches in der Schwerpunktprofessur für Psychopathologie und Neuropsychologie und der Medizinischen Informatik entwickelt und anhand aufwendiger Evaluationsstudien für den klinischen Einsatz optimiert wurde (vgl. Mehlitz et al., 1998; Mehlitz, 2004; Weniger \& Irle, 2006). 
Im Rahmen der vorliegenden Studie durchliefen alle Testpersonen ein „virtuelles Labyrinth“ zur Erfassung der egozentrischen Navigationsleistung sowie einen naturalistisch gestalteten ,virtuellen Park“ zur Erfassung der allozentrischen Navigationsleistung. Die vorliegende Arbeit stellt im Hinblick auf methodische Parameter eine Replikation der Studien von Märtens (2002) sowie Weniger \& Irle (2006) dar, die mit den gleichen virtuellen Testumgebungen und dem gleichen MRT-Scanner arbeiteten. Eine ähnliche Gestaltung findet sich bei Aguirre et al. (1997, 1998), Gillner \& Mallot (1998) und Waller et al. (1998). Im Folgenden sollen die methodische und die technische Realisierung der beiden virtuellen Testumwelten genauer beschrieben werden.

\subsubsection{Methodische Realisierung}

\subsection{Das , virtuelle Labyrinth““}

Das virtuelle Labyrinth war als einfaches Gangsystem gestaltet, bei dem etwa drei Meter hohe Wände bei einer Kopfhöhe von 1,80 Meter eine Übersicht verhinderten (vgl. Abbildungen 4a \& 4b). Die Testpersonen bewegten sich in der Mitte von zwei hellgelb erscheinenden Steinwänden unter einem blauen Himmel. Abgesehen von dem Startpunkt, der durch einen roten Kreis markiert war, befanden sich in diesem Wegenetz keinerlei Orientierungshilfen wie Objekte oder Landmarken. Das Labyrinth bestand aus insgesamt sechs Kreuzungen und sieben Sackgassen, an deren Ende sich jeweils ein Topf befand. Alle Kreuzungen erschienen identisch, egal, aus welcher Richtung sich die Versuchsperson näherte, den Zielpunkt stellte ein einziger mit Gold gefüllter Topf dar. Ein vergleichbarer Aufbau findet sich bei Ruddle et al. (1997; 1998), Aguirre et al. (1998), Waller et al. (1998) und Grön et al. (2000).

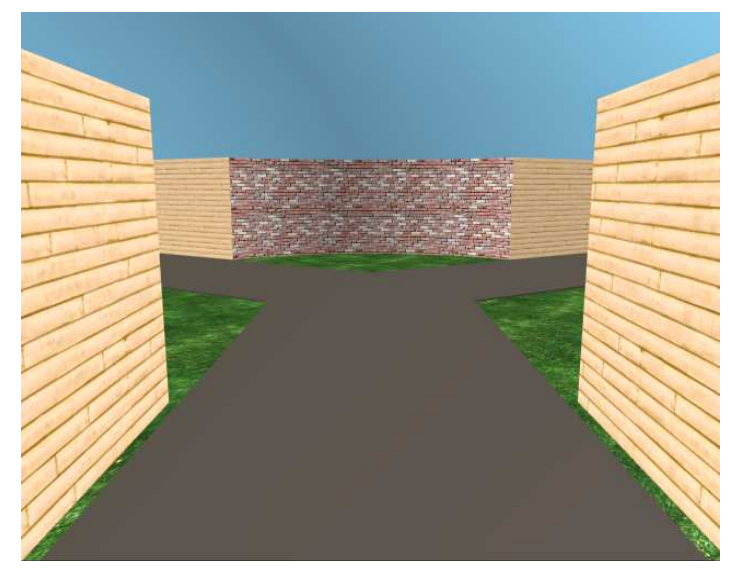

Abbildung 4a: Das Labyrinth aus Sicht der Testperson

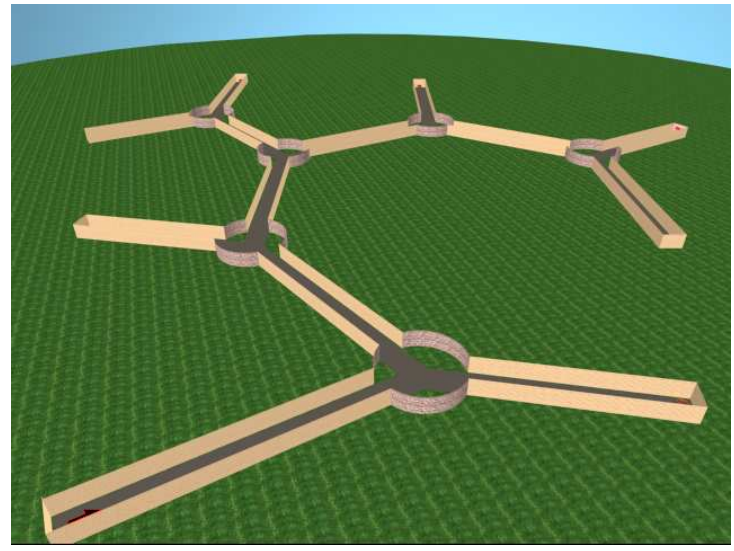

Abbildung 4b: Das Labyrinth aus exozentrischer Sicht 
Anzumerken ist, dass die Testpersonen $\mathrm{zu}$ keinem Zeitpunkt einen exozentrischen Überblick über das jeweilige Wegenetz erhielten. Die Navigation erfolgte ausschließlich innerhalb des Wegenetzes.

Das virtuelle Labyrinth diente der Erhebung der egozentrischen Navigationsleistung. Da keine externen, visuellen Hinweisreize zur Verfügung standen, mussten sich die Probanden bei der Navigation im Wesentlichen auf propriozeptive Reize (wie z.B. die eigene Kopfhaltung, Blickrichtung, Körperposition oder Bewegung) verlassen (vgl. Burgess et al., 2001a,b; Hartley et al., 2003).

\subsection{Der, ,virtuelle Park“}

Der virtuelle Park (vgl. Abbildungen 5a \& 5b) war im Gegensatz zum Labyrinth naturalistischer gestaltet: Er war mit verschiedenen Objekten und Landschaftsmerkmalen ausgestattet, anhand derer sich die Testpersonen orientieren konnten (ähnlich wie bei Distler et al., 1998; Gillner \& Mallot et al., 1998; Hartley et al., 2003). Zu diesen Landmarken zählten eine Bergkette, verschieden gestaltete Häuser, Bäume, Autos, Zäune, Kunstobjekte, Fußgängerbrücken sowie ein Spielplatz. Das ganze Parksystem wurde von einem Fluss durchzogen, der in einen See mündete. Die einzelnen Kreuzungen konnten demnach durch charakteristische visuelle Hinweisreize voneinander unterschieden werden. Kleinere Hügel innerhalb des Parks fungierten als Sichtschutz, um einzelne Bereiche voneinander abzugrenzen und eine Komplettübersicht zu verhindern. Der Park war aus insgesamt neun Weggabelungen und 11 Sackgassen aufgebaut. In jeder der Sackgassen befand sich ein Topf, der bis auf eine Ausnahme - den Zielpunkt - leer war.

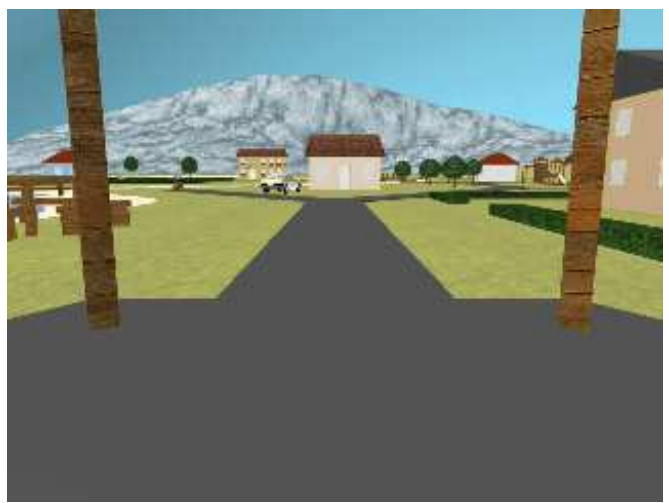

Abbildung 5a: Der Park aus Sicht der Testpersonen

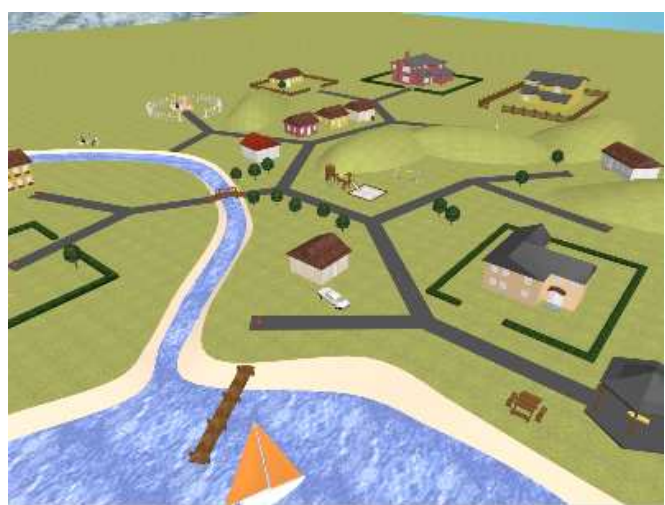

Abbildung 5b: Der Park aus exozentrischer Sicht

Im Gegensatz zum virtuellen Labyrinth erfasste die virtuelle Parklandschaft die visuellräumliche Navigationsleistung im allozentrischen Raum, bei der räumliche Konfigurationen unter Einbeziehung von Objekten aus der Umgebung und deren Relation 
zueinander erlernt werden. Im virtuellen Park erfolgt die Navigation primär auf der Grundlage externer, visueller Landmarken (wie z.B. Bäume, Häuser oder Zäune), anhand derer auch die Bildung einer mentalen Landkarte möglich ist (vgl. Aguirre et al., 1998; Gillner \& Mallot, 1998; Hartley et al., 2003)

Mit ihrer unterschiedlichen Konzeptualisierung provozierten die beiden virtuellen Testumgebungen also die Nutzung unterschiedlicher Informationsmodalitäten zur ego- und allozentrischen Navigation. Tabelle 8 stellt die wesentlichen Charakteristika der beiden Testumgebungen noch einmal kontrastierend nebeneinander:

Tabelle 8: $\quad$ Charakteristika der virtuellen Umwelten

\begin{tabular}{|c|c|c||c|}
\hline$\underline{\text { Virtuelle }}$ & $\begin{array}{c}\text { Erfasste } \\
\text { Navigationsleistung }\end{array}$ & $\frac{\text { Informations- }}{\text { modalität }}$ & $\frac{\text { Konzeption Testaufgabe }}{\text { (virtuelle Umwelt) }}$ \\
\cline { 2 - 5 } ,Labyrinth“ & Egozentrisch & $\begin{array}{c}\text { Propriozeptiv } \\
\text { - muskulär } \\
\text { - vestibulär } \\
\text { - interozeptiv }\end{array}$ & $\begin{array}{l}\text { - einfaches Gangsystem } \\
\text { - keine Landmarken } \\
\text { - Orientierung ausschließlich } \\
\text { anhand propriozeptiver cues } \\
\text { - nie: exozentrischer Überblick }\end{array}$ \\
\hline „Park“ & Allozentrisch & $\begin{array}{c}\text { Visuell } \\
\text { (Landmarken) }\end{array}$ & $\begin{array}{l}\text { - verschiedene Landmarken } \\
\text { (Berge, Häuser, Bäume etc. } \\
\text { - Orientierung anhand externer, } \\
\text { visueller cues möglich } \\
\text { - nie: exozentrischer Überblick }\end{array}$ \\
\hline
\end{tabular}

\subsection{Anzahl, Dauer und Präsentationsform der Testdurchläufe}

Aufgabe der Probanden war es, einen mit Gold gefüllten Topf innerhalb der virtuellen Testumgebung aufzufinden und sich die Route einzuprägen, um das Ziel in nachfolgenden Durchgängen effizienter wiederfinden zu können.

$\mathrm{Zu}$ diesem Zweck durchliefen die Probanden jede der beiden virtuellen Umwelten zwischen drei und fünf Mal. Dabei richteten sich Anzahl und Dauer der einzelnen Trials nach dem Lernerfolg der Versuchsperson: Ein Testdurchgang dauerte maximal 300 Sekunden und wurde bei Erreichen dieses Zeitlimits automatisch abgebrochen. Trials wurden ebenfalls beendet, wenn die Probanden das Ziel, also den mit Gold gefüllten Topf, gefunden hatten. Gelang es einer Versuchsperson, den "Schatz“ in zwei aufeinander folgenden Durchgängen fehlerfrei, also ohne Umwege, wiederzufinden, wurde die Testung 
beendet, da das Lern-Kriterium, eine gute Orientierungsfähigkeit innerhalb der virtuellen Umwelt als erreicht angesehen wurde. Ein Abbruch nach nur zwei Testdurchgängen erfolgte ebenfalls, wenn sich die Versuchspersonen orientierungslos in der virtuellen Landschaft bewegten, also kein Lernerfolg beobachtbar war.

Zusammengefasst wurde ein Testdurchgang/eine Versuchsreihe also beendet, wenn das Zeitlimit oder die Maximalzahl von fünf Trials erreicht waren, das Ziel zweifach fehlerfrei gefunden wurde oder wenn sich auch nach zwei Trials kein Lernerfolg nachweisen ließ. Der maximale „Aufenthalt“ in einer der Virtuellen Realitäten betrug bei fünf Durchgängen a 300 Sekunden ${ }^{18} 1500$ Sekunden, die minimale Zeit, die in der virtuellen Umwelt verbracht wurde, lag bei zwei Trials a` 300 Sekunden bei insgesamt 600 Sekunden. Mit einer Versuchszeit von maximal 25 Minuten pro Testbedingung (Labyrinth vs. Park) umfasste die vorliegende Testaufgabe also einen engen Zeitrahmen, der auch von Patienten mühelos bewältigt werden konnte, ohne dass mit größeren kognitiven Ermüdungserscheinungen zu rechnen war.

Um Reihenfolgeeffekte auszugleichen, wurden die beiden Testbedingungen VR-Labyrinth und VR-Park in randomisierter Reihenfolge präsentiert. Sollte sich die Navigationsleistung in den beiden virtuellen Landschaften unterscheiden, so konnte dies nicht auf Lerneffekte zurïckgeführt werden.

\subsubsection{Technische Realisierung ${ }^{19}$ :}

Zur Realisierung virtueller Testumgebungen sind im Wesentlichen zwei Komponenten notwendig: Die „Ausgabe-“ beziehungsweise „Anzeigesysteme“ präsentieren dem Benutzer die relevanten visuellen, akustischen beziehungsweise taktilen Reize der virtuellen Umgebung. Die „Eingabesysteme“ erfassen hingegen die Verhaltensweisen des Probanden wie zum Beispiel Körper-, Kopf-, Hand- oder Augenbewegungen, anhand derer eine Adaptation der virtuellen Testumgebung erfolgt. Im Folgenden sollen die Aus- und Eingabesysteme der aktuellen Arbeit kurz skizziert werden; eine Darstellung alternativer Varianten findet sich unter 7.5.1.1..

\footnotetext{
${ }^{18}$ Da die „familarity“ ein wichtiger Moderator der Navigationsleistung ist (Waller et al., 1998) erhielten die Testpersonen neben mehreren Trainingstrials relativ lange Explorationszeiten von insgesamt fünf Minuten pro trial (vgl. Ruddle et al., 1997, 1998). Längere Trialzeiten wurden nicht ausgewählt, da sie das Auftreten von Nebenwirkungen wie der simlator sickness begünstigen und somit die Testergebnisse negativ beeinflussen können (vgl. Carlin et al., 1997).

${ }^{19}$ Ergänzende Informationen finden sich unter 7.3.1. \& 7.5.1.. Zudem findet sich eine genaue Darstellung aller technischen Komponenten bei Mehlitz, 2004 und in vergleichbaren Arbeiten (siehe z.B. Mehlitz et al., 1998; van Veen et al., 1998).
} 


\subsection{Ausgabesysteme: Das Head Mounted Display}

Als wesentliches Ausgabesystem zur Präsentation der virtuellen Umwelten diente ein „Head Mounted Display“, kurz „HMD“. Bei diesem handelt es sich um eine helmartige VR-Komponente (vgl. Abbildung 6), mit deren Hilfe die gewünschten Umwelten - hier VR-Labyrinth und VR-Park - mittels zweier LCD-Bildschirme stereoskopisch präsentiert werden, um einen 3-dimensionalen Wahrnehmungseindruck beim Beobachter zu erzeugen. Innerhalb aufwendiger Evaluationsstudien konnte gezeigt werden, dass durch den Einsatz eines HMD äußerst realistische virtuelle Umwelten erzeugt werden können, die alle unter 1.2.2.2.2. diskutierten Vorzüge von VR-Paradigmen realisieren (vgl. Carrozzo \& Lacquaniti, 1998; Mehlitz, 2004). Da ein HMD zu einem höheren Immersionsgefühl und einem höheren sense of presence führt als zum Beispiel die Präsentation am Computerbildschirm, entscheiden sich viele Autoren (z.B. Rothbaum et al., 1996; Aguirre et al., 1997; Carlin et al., 1997; Distler et al., 1998; Gillner \& Mallot, 1998; Ku et al., 2004; Mühlberger et al., 2005) - und auch die vorliegende Arbeit - für die Wahl dieser VR-Komponente.

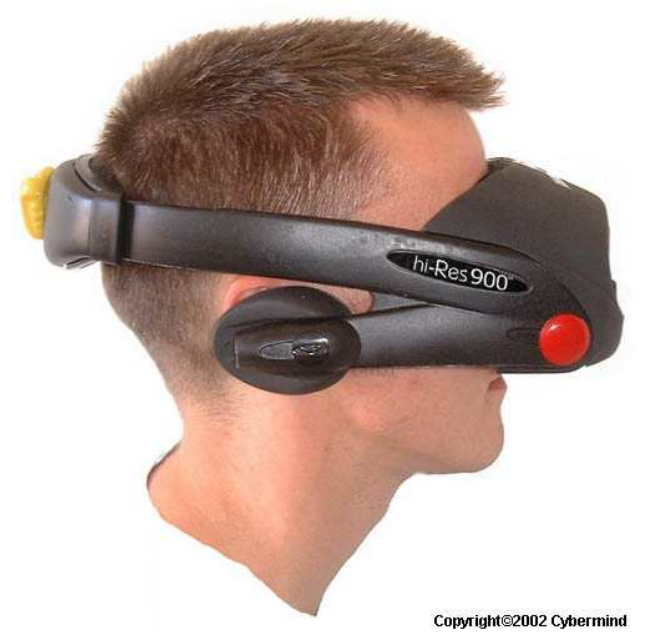

Abbildung 6: Das Head Mounted Display (HMD)

Das aktuell verwendete HMD (Sony Glasstron) besitzt einen hohen Tragekomfort bei einem geringen Gewicht von nur 180g, eine hohe Auflösung von 800 x 255 Pixeln sowie ein durch die Anzeige abgedecktes Gesichtsfeld (FOV) von etwa 35 ${ }^{\circ}$. Es zeichnet sich durch eine hohe graphische Auflösung, Farbtreue sowie durch eine hohe Benutzerfreundlichkeit aus (vgl. Mehlitz, 2004). 


\subsection{Eingabesysteme: Die Computer- und Versuchssteuerungssysteme}

Als Steuer- und Kontrollrechner wurde eine Workstation auf Basis des Betriebssystems Windows NT ausgewählt (Pentium-based PC, 233MHz, 128 MB RAM, 2 Voodoo2-Addon graphic cards, 4 MB Texture Memory). Die dreidimensionalen Testumgebungen wurden mit Hilfe des Visualisierungsprogramms Kinetix 3D Studio Max R2 entworfen. Zur Programmierung der Aufgaben dienten die Programme Realimation VSG 4.4. und Microsoft C++. Eine Auflistung der Hard- und Softwarekomponenten findet sich unter 7.3.1..

Zur Navigation der Probanden innerhalb der virtuellen Umwelten fungierten die vier Pfeiltasten einer vor ihnen platzierten Computertastatur. Eine Steuerbewegung nach vorne führte dabei zu einer frontalen Fortbewegung, eine Steuerbewegung nach hinten zu einer Drehung um 180 Grad. Steuerungen nach rechts und links führten zu der jeweils entsprechenden Drehbewegung. Somit war es der Testperson innerhalb der virtuellen Umwelten möglich, sich in einem Raum von $360 \mathrm{Grad}$ zu orientieren (vgl. Aguirre et al., 1997; Grön et al., 2000; Burgess et al., 2001a; Ku et al., 2003).

Ähnlich wie in vergleichbaren Arbeiten (Aguirre et al., 1997, Ruddle et al., 1998) zeigte sich auch in der Evaluationsstudie von Mehlitz (2004) zu dem aktuell verwendeten Testsystem, dass die Probanden nach einer kurzen Trainingszeit keinerlei Probleme in der Umsetzung der virtuellen Navigation mittels HMD als Ausgabe- und Computertastatur als Eingabesystem hatten.

\subsection{Operationalisierung der abhängigen Variablen}

\subsection{1. Überblick}

Die visuell-räumliche Navigationsleistungen der Probanden im egozentrischen (AV1) und allozentrischen (AV2) Raum wurden als zentrale abhängige Variablen erfasst. Um eine Assoziation der Navigationsleistung mit parietalen (und hippocampalen) Hirnstrukturen überprüfen zu können wurde des Weiteren ein MRT-Scan erhoben (AV3). Zur differenzierteren Erfassung kognitiver Defizite kamen zudem verschiedene neuropsychologische Testverfahren (AV4; WMS-R; TMT A/B) zum Einsatz. Abbildung 7 gibt einen Überblick über die abhängigen Variablen der vorliegenden Arbeit. 


\section{AV1: Visuell-räumliche Navigationsleistung im egozentrischen Raum (Virtuelle Realität: ,Labyrinth“) \\ AV2: $\quad$ Visuell-räumliche Navigationsleistung im allozentrischen Raum (Virtuelle Realität: „Park“") \\ AV3: $\quad$ Parietale (und hippocampale) Hirnvolumina (MRT-Scans) \\ AV4: $\quad$ Neuropsychologische Testleistungen (WMS-R, TMT A/B)}

\section{Abbildung 7: Abhängige Variablen}

\subsubsection{Erfassung der ego- und allozentrischen Navigationsleistung}

Die beiden Virtuellen Realitäten „VR-Park“ und „VR-Labyrinth“ erfassen durch ihre unter 3.2.2.1. beschriebenen Konzeptionen mit der ego- und allozentrischen Navigation zwei unterschiedliche visuell-räumliche Kognitionsleistungen. Zur Erreichung einer maximalen Testsensitivität wurden diese über verschiedene Indikatoren operationalisiert: Die Fehlerzahl, die benötigte Zeit und den Lerngewinn (vgl. O’Donnell et al., 1996; Ruddle et al., 1997; Distler et al., 1998; Maguirre, 1998; Waller et al., 1998; Skelton et al., 2000; Ku et al., 2003; Weniger \& Irle, 2006). Abbildung 8 gibt einen Überblick.

\section{1),,Fehlersumme I“ $\quad \sum$ der Fehler über alle Trials nach erstmaligem Finden des „Schatzes“ \\ 2),Fehlersumme II““ $\sum$ der Fehler über alle durchlaufenen Trials \\ 3)“'Zeit I“" durchschnittliche Zeit bis zur Zielfindung nach erstem erfolgreichen Trial \\ 4), ,Zeit II“ $\quad \sum$ der Zeit, über alle durchlaufenen Trials \\ 5)“Lernkurve I“" Differenz der Zeit bis zur Zielfindung zwischen erstem erfolgreichen Trial ( $\leq 300$ Sekunden) und letztem Trial \\ 6)"Lernkurve II" $\quad$ Differenz der Zeit bis zur Zielfindung zwischen erstem erfolgreichen Trial (Summe über alle bis dahin durchgeführten Trials) und letztem Trial}

Abbildung 8: Indikatoren der visuell-räumlichen Navigationsleistung

Insgesamt wurden sechs Indikatoren definiert: Die beiden „Fehlersummen“ bildeten sich aus der Addition aller in der virtuellen Umwelt begangenen Fehler, wobei „Fehler“ als Entscheidung für eine suboptimale Route an der jeweiligen Weggabelung definiert wurde (vgl. Hartley et al., 2003). „Fehlersumme I“ bezog ausschließlich Fehlentscheidungen ein, die nach der ersten erfolgreichen Zielfindung begangen wurden, also Fehler, die der jeweilige Proband unter optimalem Lernerfolg hätte vermeiden können. „Fehlersumme II“ 
addierte hingegen die Fehlentscheidungen über alle Trials hinweg, unabhängig davon, ob das Ziel bereits gefunden wurde oder nicht.

Analog dazu wurde mit dem Indikator „Zeit I“ die durchschnittliche Sekundenzahl erfasst, die die Probanden nach erster erfolgreicher Zielfindung in den nachfolgenden Durchgängen benötigten. Der Indikator ,Zeit II“ basierte hingegen auf der über alle Trials summierten Zeit, die sich die Probanden in der Virtuellen Realität aufhielten.

Auch der Lernerfolg wurde auf zwei Arten operationalisiert: „Lernkurve I“ verglich die benötigte Zeit des ersten erfolgreichen Trials mit der Zeit des letzten erfolgreichen Trials. „Lernkurve II“ bezog hingegen auch mit ein, ob die Probanden das Ziel bereits im ersten Testdurchgang gefunden hatten oder nicht: Hier wurde nicht nur das Erfolgstrial als Ausgangswert genutzt, sondern die insgesamt/über alle Trials summierte Zeit bis zur ersten Zielfindung.

Ergänzend zur Navigationsleistung im ego- und allozentrischen Raum wurde mit Hilfe eines Rekognitionsbogens im Anschluss an die virtuelle Testaufgabe erfasst, ob die Probanden das durchlaufene Wegenetz wiedererkennen konnten. Zudem sollten die Testpersonen innerhalb eines Nachbefragungsbogens angeben, als wie schwierig sie die jeweilige Navigationsaufgabe empfunden hatten und welche Strategie sie zum Wiederfinden des Zielpunktes verwandten. Der Rekognitions- und der Nachbefragungsbogen sind unter 7.2.4. zu finden.

\subsubsection{Erfassung der hirnanatomischen Variablen}

\subsubsection{Parameter und Aufnahmetechnick zur Erhebung der MRT-Scans}

Alle hirnanatomischen Daten wurden in Form von 3-D-Datensätzen mit Hilfe eines Magnetresonanztomographen in der Abteilung Röntgendiagnostik III des Universitätsklinikums Göttingen erhoben. Zur Erstellung der T1-gewichteten MRTBilddatensätze kam ein 1,5 Tesla Philips Gyroscan Gerät mit einer Pulsrepetitionszeit (TR) von $24.0 \mathrm{~ms}$ und einer Echozeit $(\mathrm{TE})$ von $6.0 \mathrm{~ms}$ zur Anwendung. Die dreidimensionalen Datensätze bestanden aus 256 lückenlos aneinander gereihten sagittalen Schichten, mit einer jeweils 1,0 mm betragenden Schichtdicke und einem Sichtfeld von $256 \mathrm{~mm}$. Der Aufnahmewinkel betrug $30^{\circ}$, die geometrische Auflösung lag bei 256 x 256 Pixel (vgl. Lange, 2003; Weniger \& Irle, 2006). 


\subsubsection{Auswertungsprozedere}

\subsection{Software „Curry“6}

Die digitalisierten magnetresonanztomographischen Daten zur Rekonstruktion dreidimensionaler Schichtbilder wurden zunächst auf das klinikumseigene Intranetz transferiert und nachfolgend auf eine Workstation der Abteilung Psychopathologie und Neuropsychologie übertragen. Im Anschluss erfolgte die hirnanatomische Volumetrierung der „Regions of Interest“ (ROI) mit Hilfe des Softwarepakets „Curry“ (version 4.5TM; Compumedics, Melbourne, Australia). „Curry“ ermöglicht die simultane, dreidimensionale Darstellung des Gehirns in coronaler, sagittaler und axialer Ebene und erlaubt so eine präzise Identifikation und manuelle Einzeichnung der interessierenden Hirnareale (vgl. Abbildung 9).
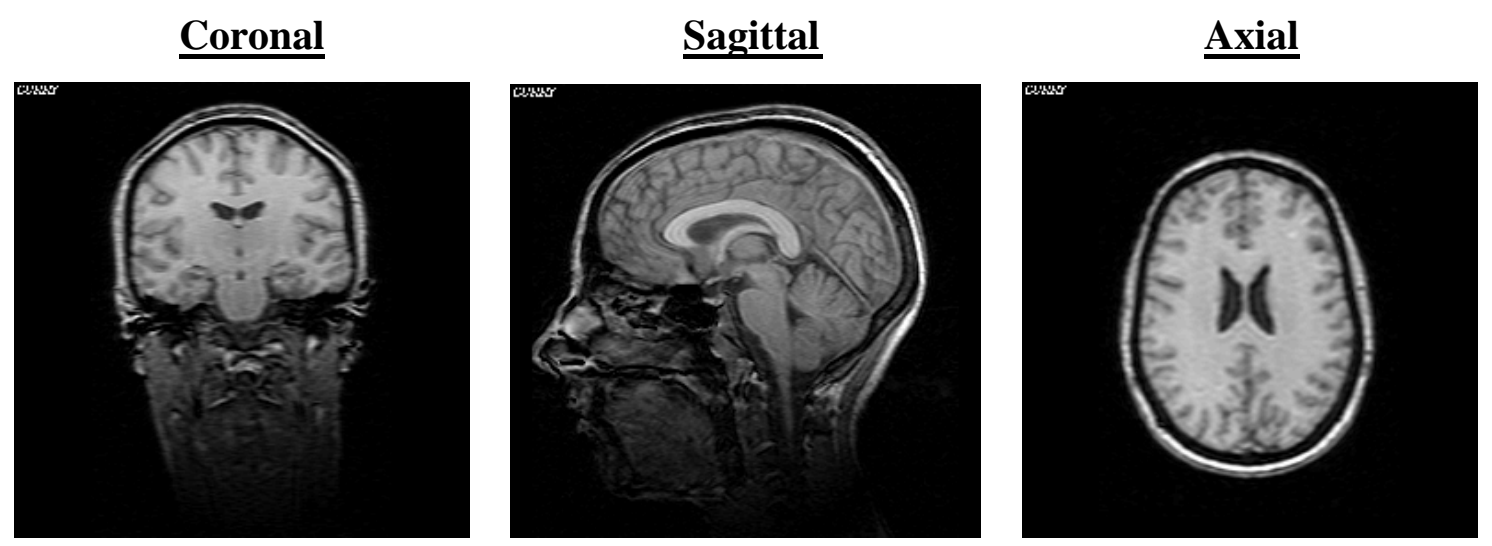

Abbildung 9: Dreidimensionale Rekonstruktion der MRT-Schichtbilder

\subsection{Hirnanatomische Grenzlinien/Manuelle Segmentierung}

Nachdem die Daten in $1.0 \mathrm{~mm}$ dicke Schichtbilder umformatiert worden waren, erfolgte die Berechnung der verschiedenen parietalen und hippocampalen Cortexvolumina mit Hilfe eines halbautomatisierten Auswertungsalgorithmus: Für jeden einzelnen Datensatz wurde eine individuelle Cortexschwelle festgelegt, die die Grenze zwischen Hirngewebe und Umgebung (Liquor, Knochen...) definierte. Im Anschluss erfolgte die manuelle Einzeichnung der interessierenden ROI. Zu diesem Zweck wurden die Grenzen des jeweiligen Areals 3-dimensional, also in coronaler, sagittaler und axialer Ansicht, eingezeichnet. Als Grenzlinien dienten dabei verschiedene Sulci, die den Parietallappen und den Hippocampus charakteristisch von umliegendem cortikalen Gewebe abgrenzen (vgl. Nieuwenhuys et al., 1978; Talairach \& Tournoux, 1988). Vorteilhaft bei der Auswahl der Sulci ist, dass sich vor allem in ihnen die substantielle, interindividuelle morphologische Variabilität menschlicher Cortices widerspiegelt; interindividuelle 
cortikale Unterschiede sollten sich mit den so definierten Grenzlinien also deutlich beobachten lassen (vgl. Rademacher et al., 1992).

Abbildung 10 zeigt die hirnanatomischen Grenzlinien, anhand derer die Segmentierung des Parietallappens sowie seiner Subareale erfolgte (vgl. Irle et al., 2005). Da hippocampale Areale in der vorliegenden Studie nur explorativ untersucht wurden, wird ihre Volumetrierung nicht erläutert. Eine Darstellung findet sich bei Lange (2003) und Irle et al. (2005).

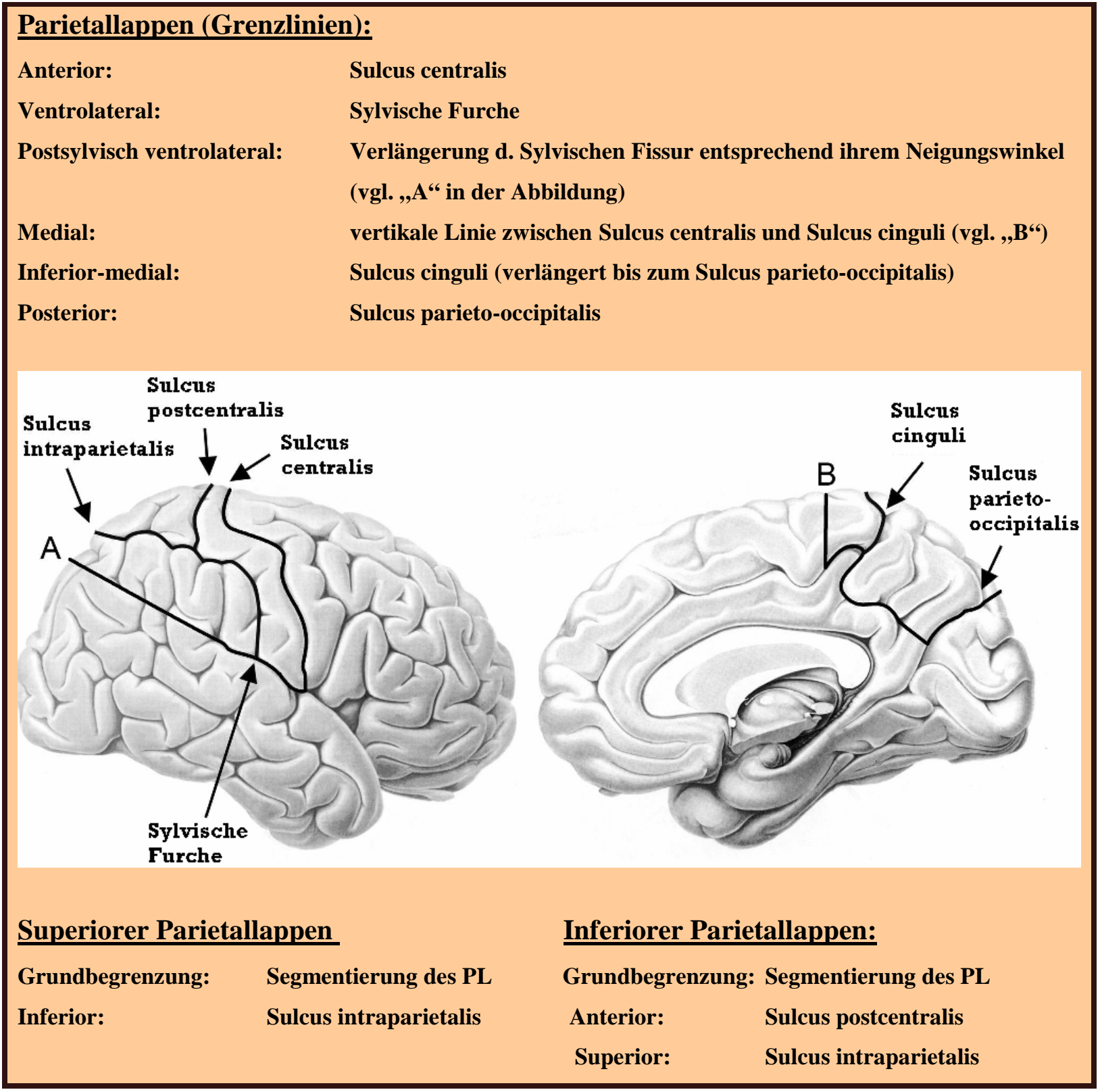

Abbildung 10: Grenzlinien des Parietallappens \& parietaler Subareale 
Wie aus Abbildung 10 ersichtlich, diente der Sulcus centralis als anteriore Begrenzung zur Trennung des Parietallappens von frontalen Arealen; der Sulcus parieto-occipitalis fungierte als Grenzlinie zwischen parietalen und okzipitalen Arealen. Auf medialer Ebene wurde eine vertikale Linie zwischen dem Sulcus centralis und dem Sulcus cinguli gezogen, als inferior-mediale Begrenzung diente eine Markierung des Sulcus cinguli sowie dessen Verlängerung bis zum Sulcus parieto-occipitalis. Als ventrolaterale Begrenzungslinie wurde die sylvische Fissur definiert; eine posteriore Verlängerung der sylvischen Fissur entsprechend ihrem Neigungswinkel diente als postsylvisch ventrolaterale Grenzlinie. Die manuelle Segmentierung erfolgte auf coronalen Schichtbildern, indem eine Verbindungslinie zwischen der sylvischen Fissur und dem Sulcus centralis sowie zwischen der posterioren Verlängerung der sylvischen Fissur und dem Sulcus cinguli gezogen wurde.

Zur Segmentierung des superioren und des inferioren Parietallappens wurde dieselbe Grundbegrenzung wie für die Segmentierung des globalen Parietallappens herangezogen. Die Trennung der beiden Subareale erfolgte anhand des Sulcus intraparietalis, zudem wurde als anteriore Grenzlinie des inferioren Parietallappens der Sulcus postcentralis definiert.

\subsubsection{Neuropsychologische Testverfahren}

Zur Erfassung der allgemeinen kognitiven Leistungsfähigkeit sowie einiger neuropsychologischer Basisfunktionen wurde eine Reihe von konventionellen, standardisierten Testverfahren durchgeführt. Die Auswahl der Verfahren orientierte sich dabei an vergleichbaren Studien mit ähnlichen Fragestellungen zu neuropsychologischen Testleistungen und Hirnmorphologie bei (schizophrenen) Testpersonen (vgl. Maguire et al., 1996; Roob, 2002; Sanfilipo et al., 2002; Ku et al., 2003). Tabelle 9 gibt einen Überblick über die verwandten Verfahren und die mit ihnen erfassten kognitiven Funktionen. $\mathrm{Zu}$ erwähnen ist, dass der HAWIE-R im vorliegenden Design dem Patientenscreening diente, allerdings nicht in die Forschungshypothesen einging. 
Tabelle 9: $\quad$ Neuropsychologische Testverfahren der aktuellen Studie

\begin{tabular}{|c|c|c|}
\hline Testverfahren & $\underline{\text { Subtests }}$ & Erfasste kognitive Funktionen \\
\hline $\begin{array}{c}\text { HAWIE-R } \\
\text { Hamburg-Wechsler- } \\
\text { Intelligenztest für } \\
\text { Erwachsene-Revision } \\
\text { (Tewes, 1991) }\end{array}$ & $\begin{array}{l}\text { Gesamttest } \\
\underline{\text { Verbalteil }} \\
\text { - Allgemeines Wissen } \\
\text { - Gemeinsamkeiten finden } \\
\text { Handlungsteil } \\
\text { - Bilder ergänzen } \\
\text { - Mosaiktest }\end{array}$ & $\begin{array}{c}\text { intellektuelles Leistungsniveau } \\
\text { Allgemeinwissen } \\
\text { Verbal-logische semantische Konzeptbildung } \\
\text { Erkennen wesentlicher visueller Details } \\
\text { Visuo-konstruktive Fähigkeiten }\end{array}$ \\
\hline $\begin{array}{c}\text { WMS-R } \\
\text { Wechsler Memory } \\
\text { Scale Revised } \\
\text { (Wechsler, 1987) }\end{array}$ & $\begin{array}{l}\text { - Digit Span Forward } \\
\text { - Digit Span Backward } \\
\text { - Visual Memory Span fwd } \\
\text { - Visual Memory Span bwd } \\
\text { - Logical Memory I } \\
\text { - Logical Memory II } \\
\text { - Visual Reproduction I } \\
\text { - Visual Reproduction II }\end{array}$ & $\begin{array}{c}\text { Verbale Gedächtnisspanne (KZG) } \\
\text { Verbale Gedächtnisspanne (KZG) } \\
\text { Visuelle Gedächtnisspanne (KZG) } \\
\text { Visuelle Gedächtnisspanne (KZG) } \\
\text { Verbales KZG } \\
\text { Verbales LZG } \\
\text { Visuelles KZG } \\
\text { Visuelles LZG }\end{array}$ \\
\hline $\begin{array}{c}\text { TMT-A; TMT-B } \\
\text { Trail Making Test } \\
\text { (Reitan, 1992) }\end{array}$ & $\begin{array}{l}\text { - TMT- Version A } \\
\text { - TMT- Version B }\end{array}$ & $\begin{array}{l}\text { Visuo-motorische Aktionsfähigkeit } \\
\text { Visuo-motorische Aktionsfähigkeit und } \\
\text { Aufmerksamkeitsshift }\end{array}$ \\
\hline
\end{tabular}

KZG = Kurzzeitgedächtnis; LZG = Langzeitgedächtnis; fwd = forward; bwd = backward.

\subsubsection{HAWIE-R}

Der Hamburg-Wechsler-Intelligenztest für Erwachsene-Revision (Tewes, 1991) erfasst das allgemeine intellektuelle Leistungsniveau der Probanden und dient somit der Untersuchung des allgemeinen geistigen Entwicklungsstandes (vgl. Brähler et al., 2002a). Der HAWIE-R ist die deutschsprachige Version des WAIS-III (Wechsler, 1981) und ist sowohl im wissenschaftlichen Setting, als auch im klinischen Rahmen ein gut etabliertes und weit verbreitetes Testverfahren.

Da es sich bei dem aus 11 Untertests bestehenden HAWIE-R um ein sehr aufwendiges Testsystem handelt, wurde in der vorliegenden Arbeit eine aus vier Subtests bestehende Kurzversion durchgeführt, die als valider Schätzer für die Erfassung der allgemeinen 
kognitiven Leistungsfähigkeit akzeptiert wird. Die Untertests Allgemeines Wissen, Gemeinsamkeiten finden, Bilder ergänzen und Mosaiktest gelten als beste Schätzer für Verbal- und Handlungs-IQ, da sie sich durch hohe Interkorrelationen mit den anderen sieben Subtests auszeichnen und zudem eine hohe Korrelation mit dem Gesamt-IQ bei Durchführung des Gesamttests aufweisen (Tewes, 1991). ${ }^{20}$

Obwohl der HAWIE-R aufgrund seiner eingeschränkten Standardisierung offensichtliche Nachteile bezüglich Objektivität - und somit auch bezüglich Reliabilität und Validität - in sich birgt, ist er ein in der Praxis hoch bewährtes und häufig genutztes Verfahren und fand daher auch in der vorliegenden Arbeit Verwendung. Hier erfüllte er zwei wesentliche Funktionen: Zum einen diente er der Erfassung der allgemeinen kognitiven Leistungsfähigkeit im Sinne eines Screeningverfahren. Testpersonen mit einem Intelligenzquotienten unter 80 Punkten wurden von der Teilnahme ausgeschlossen. Zum anderen wurde der HAWIE-R als Parallelisierungsverfahren herangezogen. Mit ihm wurde ein ähnliches Leistungsniveau in den zu vergleichenden Probandengruppen sichergestellt um zu gewährleisten, dass eine überlegene Leistung der Kontrollprobanden in der „Virtuellen Realität“ durch höhere Fähigkeiten in der visuell-räumlichen Informationsverarbeitung bedingt war und nicht durch eine allgemein höhere Intelligenz.

\subsubsection{WMS-R}

Die Wechsler Memory Scale-Revised (Wechsler, 1987) ist ein bewährtes Verfahren der klinischen Neuropsychologie zur Erfassung verschiedener, in der Gedächtnisforschung etablierter mnestischer Teilbereiche. Alle 14 Subtests erfassen explizite Gedächtnisleistungen, variieren in ihrer Komplexität und Abrufmodalität (freier versus geleiteter Abruf, Rekognition) und dienen zur Bewertung des verbalen und visuellen Kurzzeit- und Langzeitgedächtnisses. Wie aus Tabelle 9 ersichtlich, wurde in der vorliegenden Arbeit eine verkürzte Version des WMS-R, bestehend aus acht Subtests, durchgeführt.

Die WMS-R zeichnet sich mit hohen Reliabilitäten zwischen $r=.70-.90$ sowie mit einer hohen Konstruktvalidität - die an verschiedenen neurologischen, neurochirurgischen und psychiatrischen Probanden evaluiert wurde - durch gute Testgütekriterien aus (vgl. Brähler et al., 2002b).

\footnotetext{
${ }^{20} \mathrm{Da}$ der HAWIE-R in der vorliegenden Arbeit lediglich als Screeningverfahren und die WMS-R als Nebenvariable erhoben wurde, soll auf eine detaillierte Darstellung der jeweiligen Subtests verzichtet werden. Eine genaue Charakterisierung findet sich im Anhang unter 7.5.2..
} 


\subsubsection{TMT-A und TMT-B}

Der Trail Making Test A (Reitan, 1992) erfasst die einfache, visuomotorische Aktionsgeschwindigkeit der Probanden, deren Aufgabe darin besteht, 25 auf einem DINA4-Blatt verteilte Zahlen so schnell wie möglich zu verbinden. Der anspruchsvollere Trail Making Test B (Reitan, 1992) dient zudem als Indikator für die kognitive Flexibilität und die Fähigkeit der Probanden zum Aufmerksamkeitsshift; sie müssen so schnell wie möglich eine wechselnde Abfolge von Zahlen und Buchstaben miteinander verbinden.

Beide Testversionen können als globales Maß für die kognitive Informationsverarbeitungseffizienz angesehen werden, da sie das schnelle Erkennen und Verarbeiten bedeutsamer Zahlen und/oder Buchstaben sowie deren flexible Integration in eine Reihenfolge verlangen. Sie eignen sich hervorragend zur Messung des allgemeinen (cortikalen) Leistungsniveaus oder wie der Autor es ausdrückt: Sie sind ein „,widely used measure for general brain functions “ (Hogrefe, 2004, S. 264).

\subsection{Versuchsaufbau und -ablauf}

Die vorliegende Studie setzte sich aus insgesamt drei Versuchsteilen zusammen: Aus der neuropsychologischen (und gegebenenfalls psychopathologischen) Testung, aus der „Virtuellen Realität“ sowie aus der Erhebung des MRT-Scans. Die drei Testteile wurden je nach Belastbarkeit der einzelnen Probanden zu zwei bis drei Erhebungszeitpunkten innerhalb von maximal 2 Wochen durchgeführt. Im Folgenden soll der Ablauf einer „typischen“ Testsitzung kurz skizziert werden. ${ }^{21}$

\subsubsection{Aufklärung und Einverständniserklärung}

Zunächst wurden die Probanden begrüßt und darüber informiert, dass sie im Rahmen der aktuellen Studie an verschiedenen Versuchselementen teilnehmen würden, zu denen unter anderem ein MRT-Scan zählte. Den Teilnehmern wurde zugesichert, dass sie jederzeit Gelegenheit hätten, Rückfragen zu stellen, um Pausen zu bitten oder den Versuch abzubrechen. Im Anschluss an diese allgemeine Aufklärung unterschrieben die Versuchspersonen eine Einverständniserklärung, die die Freiwilligkeit ihrer Teilnahme bestätigte und das Uniklinikum berechtigte, die erhobenen Daten unter Berücksichtigung der Schweigepflicht für wissenschaftliche Zwecke zu nutzen.

\footnotetext{
${ }^{21}$ Alle Aufklärungstexte und Fragebögen sind im Anhang unter 7.2. zu finden.
} 


\subsubsection{Versuchsteil 1: Fragebögen, neuropsychologische (und psychopathologische)} Testung

$\mathrm{Zu}$ Beginn der Datenerhebung wurden die wichtigsten Kenndaten der Probanden sowie einige neuropsychologische Parameter erhoben. Zu diesem Zweck beantworteten die Probanden einen Screeningfragebogen und füllten einen soziodemographischen Fragebogen aus, in dem einige wichtige Kenndaten wie Alter, Geschlecht, und Schulbildung festgehalten wurden. Patienten wurden zudem gebeten, einige Angaben im Hinblick auf ihre Erkrankung zu machen, wie z.B. den Zeitpunkt der schizophrenen Erstmanifestation, die aktuelle Medikation und die Häufigkeit der bisherigen Hospitalisierungen (vgl. 3.1.).

Im Anschluss an die Anamnesebögen folgte eine 1-2 stündige Testsitzung, innerhalb der die Probanden unter Anleitung eines Versuchsleiters eine Kurzversion des HAWIE-R und der WMS-R sowie den TMT-A und den TMT-B bearbeiteten (AV4).

Zur Erfassung der individuellen Psychopathologie wurden bei schizophrenen Teilnehmern bereits im Vorfeld der Studie verschiedene standardisierte Testverfahren (GAF, CGI, BPRS, SAPS und SANS) durch erfahrene Psychiater des Universitätsklinikums erhoben.

\subsubsection{Versuchsteil 2: Die Virtuelle Realität}

Im Zentrum des zweiten Versuchsteils stand die Erhebung visuell-räumlicher Navigationsprozesse im egozentrischen (AV1) sowie im allozentrischen Raum (AV2).

\subsubsection{Instruktionen}

Zu Beginn des zweiten Versuchsteils wurden die Probanden anhand eines standardisierten Aufklärungstexts über Ziel und Ablauf der aktuellen Studie sowie über das zu diesem Zweck realisierte VR-Paradigma informiert (vgl. 7.2.4.1.).

Zur Erzeugung Virtueller Realität wurden die Probanden im Anschluss gebeten, das „Head Mounted Display“ (HMD) aufzusetzen, welches mit Hilfe eines flexiblen Kopfbügels an die individuelle Kopfform angepasst wurde. Zudem erfolgte eine Adaptation des HMD an das visuelle System der jeweiligen Testperson, in dem der Abstand zwischen Augen und Datenbrille so lange variiert wurde, bis keine Doppelbilder mehr zu sehen waren.

Nach Adjustierung des HMD wurden die Probanden darüber aufgeklärt, dass sie sich nachfolgend in einer virtuellen Welt orientieren sollten, innerhalb der sie sich mit Hilfe der vor ihnen positionierten Computertastatur fortbewegen könnten. Sie wurden gebeten, die Finger ihrer dominanten Hand auf die Pfeiltasten der Tastatur zu legen. Des Weiteren 
wurde erklärt, dass alle folgenden Instruktionen in schriftlicher Form über das HMD erfolgen würden. Die Probanden wurden gebeten, die einzelnen Anleitungen aufmerksam $\mathrm{zu}$ lesen und den jeweiligen Aufforderungen zu folgen. Die komplette nachfolgende Testadministration wurde datenbankbasiert umgesetzt.

Eine Darbietung der wesentlichen Instruktionen in schriftlicher Form bot den Vorteil, dass die Probanden die Geschwindigkeit der Instuktionsabfolge per Tastendruck selbst steuern und sie somit auf ihre individuelle Leistungs- und Aufnahmefähigkeit abstimmen konnten. Dies schien insbesondere im Hinblick auf die Patientengruppe bedeutsam, bei der durch schnelle, externe Instruktionen leicht Verständnisschwierigkeiten auftreten konnten. Zudem konnte durch die schriftliche Präsentation der Anweisungen eine optimale Standardisierung der Versuchsbedingungen gewährleistet werden.

Der Versuchsleiter konnte die Navigation der Probanden während des kompletten Versuchablaufs über einen zweiten Computermonitor verfolgen, auf dem die Position der Testperson innerhalb des vogelperspektivisch abgebildeten Wegenetzes kontinuierlich durch einen Pfeil visualisiert wurde.

\subsubsection{Trainingstrials}

Nach Starten des Versuchsprogramms wurde den Probanden zunächst ein Einführungstext präsentiert, in dem ihnen erklärt wurde, wie sie sich mit Hilfe der Computertastatur innerhalb der Virtuellen Realitäten fortbewegen konnten (vgl. 7.2.4.2.). Im Anschluss erhielten die Probanden Gelegenheit, sich innerhalb eines sechsminütigen Trainingstrials mit der tastaturgesteuerten Navigation in der virtuellen Umwelt vertraut zu machen. Erst wenn die Testpersonen die virtuelle Navigation sicher beherrschten und die Anforderungen korrekt verstanden hatten, wurde das Versuchsprogramm für die Testtrials gestartet.

\subsubsection{Testtrials}

Vor Präsentation des ersten Testtrials wurde den Probanden eine weitere Instruktion präsentiert. Diese klärte darüber auf, dass die wesentliche Aufgabe in den nachfolgenden Testdurchgängen darin bestehe, den kürzesten Weg zu einem Zielpunkt (dem „Goldtopf“) zu finden. Die Probanden wurden gebeten, sich so schnell und effizient wie möglich innerhalb der virtuellen Testumgebung zu orientieren und sich die Route zum „Goldtopf“ möglichst gut einzuprägen, da dieser in nachfolgenden Durchgängen wiedergefunden werden sollte. Wenn die Probanden ihre Aufgabe verstanden hatten, wurde das erste Testtrial gestartet. 
Sobald es der Versuchsperson gelungen war, den Zielpunkt zu erreichen, wurde das Testtrial gestoppt. Die Probanden wurden darüber informiert, dass ein weiterer Durchgang in derselben Testumgebung folgen würde und ihre Aufgabe darin bestehe, den Zielpunkt so schnell wie möglich wiederzufinden. Dabei konnte jeder Versuchsdurchgang von den Probanden selbstständig durch Tastendruck gestartet werden, so dass individuell lange Pausen zwischen den einzelnen Trials möglich waren.

Hatte die Versuchsperson in beiden Virtuellen Realitäten eines der unter 3.2.2.1.3. geschilderten Abbruchkriterien erreicht und somit zwischen sechs und zehn Durchgängen absolviert, endete die Testung innerhalb des VR-Paradigmas. Die Probanden wurden gebeten, einen Rekognitionsbogen sowie einen abschließenden Nachbefragungsbogen auszufüllen und wurden mit Verweis auf den letzten Testteil zur Erhebung der hirnanatomischen Daten verabschiedet.

\subsubsection{Versuchsteil 3: Magnetresonanztomographie}

Um Aussagen über eine mögliche hirnanatomische Genese kognitiver Defizite treffen zu können, wurden neben den (neuro-)psychologischen Daten die parietalen und hippocampalen Hirnvolumina der Probanden erfasst. Zu diesem Zweck diente ein Magnetresonanztomograph der Abteilung Röntgendiagnositk III des Universitätsklinikums Göttingen.

\subsubsection{Aufklärung und Einverständniserklärung}

Zunächst wurden die Testpersonen über die Funktionsweise des Magnetresonanztomographen informiert (vgl. 7.2.5.). Da viele Probanden im Zusammenhang mit dem MRT Ängste formulierten, erschien es besonders wesentlich, die Gefahrlosigkeit der Untersuchung hervorzuheben und darauf hinzuweisen, dass die Möglichkeit zum Abbruch jederzeit gegeben war, ohne dass dadurch persönliche Nachteile entstehen würden. Darüber hinaus klärte der Versuchsleiter detailliert über Dauer und Setting - wie z.B. seine eigene Positionierung während des Experiments - auf.

Die Probanden wurden gebeten, sich in den MRT-Scanner zu legen und sich während der kompletten folgenden Testung möglichst ruhig zu verhalten, da Bewegungsartefakte (wie z.B. Blinzeln) zur Verfälschung der Daten führen könnten. Um der Testperson die Möglichkeit zum Abbruch zu geben, erhielt sie einen Signalknopf, mit dem sie die Erhebung zu jedem Zeitpunkt stoppen konnte. 


\subsubsection{Erhebung der hirnanatomischen Daten}

Die Testpersonen lagen ruhig im Magnetresonanztomographen, während dieser ihren Cortex aus coronaler, sagittaler und axialer Ebene scannte und Daten zur Rekonstruktion 3-dimensionaler Schichtbilder erfasste. Insgesamt dauerte die Erhebung der Hirnanatomie circa 30 Minuten.

Am Ende des dritten Versuchsteils erhielten die Probanden abschließend Gelegenheit, Fragen zum Experiment zu stellen und ihnen wurde für ihre Teilnahme an dem Experiment gedankt.

\subsubsection{Zusammenfassung}

Tabelle 10 gibt den dargestellten Aufbau der einzelnen Versuchsteile, die jeweils erfassten Variablen, die zugrunde liegende Methodik sowie den Ort der Testung in einem abschließenden Überblick wieder:

Tabelle 10: Der Versuchsaufbau \&-ablauf

\begin{tabular}{|c|c|c|c|c|}
\hline & \multirow{2}{*}{$\frac{\text { Screening }}{\text { (Patienten) }}$} & \multicolumn{3}{|c|}{ Versuchsteil } \\
\hline & & 1 & 2 & 3 \\
\hline$\frac{\text { Erfasste }}{\text { Variablen }}$ & $\begin{array}{l}\text { Psycho- } \\
\text { pathologie }\end{array}$ & $\frac{\text { Neuropsychologie }}{(A V 4)}$ & $\begin{array}{l}\text { Visuell-räumliche } \\
\text { Navigation: } \\
\text { egozentrisch (AV1) } \\
\text { allozentrisch (AV2) }\end{array}$ & $\begin{array}{l}\frac{\text { Hirnanatomie }}{(\mathrm{AV} 3)} \\
\text { Parietallappen } \\
\text { Hippocampus }\end{array}$ \\
\hline$\frac{\text { Methodik/ }}{\text { Verfahren }}$ & $\begin{array}{l}\text { SKID } \\
\text { GAF } \\
\text { CGI } \\
\text { BPRS } \\
\text { SAPS } \\
\text { SANS }\end{array}$ & $\begin{array}{c}\text { HAWIE-R } \\
\text { WMS-R } \\
\text { TMT A \& B } \\
\\
\text { zudem: } \\
\text { - Einverständnis } \\
\text { - soziodem. FB } \\
\end{array}$ & $\begin{array}{l}\text { Virtuelle Realität } \\
\text { 1) Labyrinth } \\
\text { 2) Park } \\
\text { zudem: } \\
\text { - Rekognitions- \& } \\
\text { - Nachbefragungsbogen }\end{array}$ & MRT-Scan \\
\hline$\underline{\text { Ort }}$ & $\begin{array}{l}\text { Uniklinikum } \\
\text { Göttingen } \\
\text { (stationär) }\end{array}$ & \begin{tabular}{|c} 
Abteilung \\
Neuropsychologie \& \\
Psychopathologie \\
Uniklinikum \\
Göttingen
\end{tabular} & $\begin{array}{c}\text { Abteilung } \\
\text { Neuropsychologie \& } \\
\text { Psychopathologie } \\
\text { Uniklinikum } \\
\text { Göttingen }\end{array}$ & $\begin{array}{c}\text { Abteilung } \\
\text { Röntgendiagnostik III } \\
\text { Uniklinikum } \\
\text { Göttingen }\end{array}$ \\
\hline
\end{tabular}




\subsection{Datenanalyse}

Alle statistischen Analysen wurden mit Hilfe der Softwarepakete Statistical Package for the Social Sciences (SPSS for Windows, Version 12.0) und Statistical Analysis System (SAS for Windows, Version 8.02.) durchgeführt. Dabei gingen ausschließlich Rohwerte in die Berechnungen ein.

Das Alphaniveau wurde entsprechend gängiger Normen auf 0.05 festgelegt (vgl. Bortz, 1993). Auf eine Alpha-Adjustierung wurde in der vorliegenden Arbeit verzichtet, da klinische Studien zum einen mit einer begrenzten Stichprobenzahl auskommen müssen, die das Auftreten von Beta-Fehlern begünstigt und da das vorliegende Forschungsgebiet zum anderen durch einen noch geringen Kenntnisstand charakterisiert ist, der ein hypothesengenerierndes Vorgehen nahe legt. Diese Entscheidung folgte nicht aus statistischen sondern aus forschungspraktischen Gründen: Ein Beta-Fehler, also das Übersehen möglicherweise vorhandener neuropsychologischer und hirnanatomischer Defizite in der schizophrenen Probandengruppe hätte größere Konsequenzen als ein potentieller Alphafehler, da weitere Forschungsbemühungen in diesem Bereich möglicherweise ausblieben. 


\section{Ergebnisse}

\subsection{Zentrale Forschungshypothesen}

\subsubsection{Neuropsychologie: Visuell-räumliche Navigationsleistung}

\section{Deskription}

In der vorliegenden Untersuchung wurde die visuell-räumliche Navigationsleistung 25 schizophrener Patienten sowie 25 gesunder Testpersonen in zwei verschiedenen virtuellen Testumgebungen erfasst. Tabelle 11 gibt die Ergebnisse für den egozentrischen Raum (Bedingung „VR-Labyrinth“) sowie für den allozentrischen Raum (Bedingung „VR-Park“) vergleichend wieder.

Tabelle 11: Navigationsleistung der Probandengruppen im ego-\& allozentrischen Raum

\begin{tabular}{|c|c|c|c|c|c|c|c|c|}
\hline & \multicolumn{4}{|c|}{,VR-Labyrinth } & \multicolumn{4}{|c|}{ „VR-Park } \\
\hline $\begin{array}{l}\text { Gruppe: } \\
\text { Indikator: }\end{array}$ & \multicolumn{2}{|c|}{$\frac{\frac{\text { Schizophrene }}{\text { Patientengruppe }}}{(n=25)}$} & \multicolumn{2}{|c|}{$\frac{\begin{array}{c}\text { Gesunde } \\
\text { Kontrollgruppe }\end{array}}{(n=25)}$} & \multicolumn{2}{|c|}{$\frac{\begin{array}{c}\text { Schizophrene } \\
\text { Patientengruppe }\end{array}}{(n=25)}$} & \multicolumn{2}{|c|}{$\frac{\begin{array}{c}\text { Gesunde } \\
\text { Kontrollgruppe }\end{array}}{(n=25)}$} \\
\hline & $\underline{\text { MW }}$ & $\underline{\mathbf{s}}$ & $\underline{\text { MW }}$ & $\underline{\mathbf{S}}$ & $\underline{\text { MW }}$ & $\underline{\mathbf{s}}$ & $\underline{\text { MW }}$ & $\underline{\mathbf{s}}$ \\
\hline Fehlersumme I & 11,40 & 11,81 & 5,84 & 10,43 & 4,92 & 6,33 & 0,32 & 0,63 \\
\hline Fehlersumme II & 17,00 & 10,36 & 13,08 & 10,64 & 19,08 & 14,64 & 9,20 & 5,66 \\
\hline Zeit $\mathbf{I}^{+}$ & 183,48 & 88,12 & 130,36 & 60,84 & 165,03 & 95,14 & 92,53 & 11,37 \\
\hline Zeit $\mathrm{II}^{+}$ & 837,09 & 343,31 & 734,86 & 321,54 & 824,97 & 384,56 & 569,77 & 222,01 \\
\hline 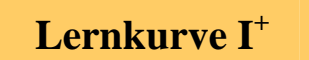 & 15,38 & 146,69 & 74,27 & 71,24 & 44,18 & 114,23 & 100,55 & 70,41 \\
\hline Lernkurve II $^{+}$ & 99,41 & 254,09 & 218,31 & 257,01 & 211,68 & 266,29 & 256,39 & 196,65 \\
\hline
\end{tabular}

MW = Mittelwert; $\mathrm{s}=$ Standardabweichung; ${ }^{+}=$Angabe in Sekunden.

Im egozentrischen Raum („VR-Labyrinth“) zeigte sich auf deskriptiver Ebene mit allen erfassten Indikatoren eine schwächere Navigationsleistung bei der schizophrenen Probandengruppe. Schizophrene Probanden begingen mehr Fehler bei der Suche nach dem Zielpunkt (MW = 11,40 bzw. 17,00 in der Patientengruppe vs. MW = 5,84 bzw. 13,08 in der Kontrollgruppe), benötigten mehr Zeit, um diesen zu erreichen (MW = 183,48 sec bzW. $837,09 \mathrm{sec}$ in der Patientengruppe vs. $\mathrm{MW}=130,36 \mathrm{sec}$ bzw. 734,86 sec in der Kontrollgruppe) und wiesen einen geringeren Lerngewinn auf als gesunde 
Kontrollprobanden (MW $=15,38$ sec bzw. 99,41 sec in der Patientengruppe vs. MW = 74,27 sec bzw. 218,31 sec in der Kontrollgruppe).

Im allozentrischen Raum (,VR-Park“) zeichnete sich ein ähnliches Bild: Auch hier wiesen die schizophrenen Probanden ein durchweg schwächeres Leistungsprofil auf als die gesunde Kontrollgruppe. Sie begingen mehr Fehler bei der Suche nach dem Zielpunkt (MW = 4,92 bzw. 19,08 in der Patientengruppe vs. $\mathrm{MW}=0,32$ bzw. 9,20 in der Kontrollgruppe), benötigten mehr Zeit, um diesen zu erreichen ( $\mathrm{MW}=165,03 \mathrm{sec}$ bzw. $824,97 \mathrm{sec}$ in der Patientengruppe vs. $\mathrm{MW}=92,53 \mathrm{sec}$ bzw. 569,77 $\mathrm{sec}$ in der Kontrollgruppe) und wiesen einen geringeren Lerngewinn auf (MW $=44,18 \mathrm{sec}$ bzw. $211,68 \mathrm{sec}$ in der Patientengruppe vs. $\mathrm{MW}=100,55 \mathrm{sec}$ bzw. 256,39 $\mathrm{sec}$ in der Kontrollgruppe).

\section{Inferenzstatistik}

Um die ego- und die allozentrische Navigationsleistung der schizophrenen Probandengruppe und der Kontrollpersonen inferenzstatistisch miteinander vergleichen zu können, wurden zweifaktorielle, multivariate Varianzanalysen (analysis of variance, ANOVAs) mit den abhängigen Variablen „Fehlersumme I und II“, „Zeit I und II“ sowie „Lerngewinn I und II“ und den jeweils zweistufigen Faktoren „Gruppe“ (schizophrene vs. gesunde Testpersonen) und „Testumwelt“ (VR-Labyrinth vs. VR-Park) durchgeführt. Tabelle 12 gibt die varianzanalytischen Kennwerte in tabellarischer Form wieder:

Tabelle 12: $\quad$ Ergebnisse der zweifaktoriellen, multivariaten ANOVAs mit den Faktoren „Gruppe“ \& „Testumwelt“

\begin{tabular}{|c|c|c|c|c|c|c|}
\hline \multirow[t]{2}{*}{ Indikator: } & \multicolumn{2}{|c|}{$\frac{\frac{\text { Haupteffekt }}{\text { „Gruppe }^{66}}}{(\mathrm{n}=25)}$} & \multicolumn{2}{|c|}{$\frac{\frac{\text { Haupteffekt }}{\text { Testumwelt }^{66}}}{(\mathrm{n}=25)}$} & \multicolumn{2}{|c|}{$\frac{\text { „Gruppe - Testumwelt“" }}{(n=25)}$} \\
\hline & $\underline{\mathbf{F}(\mathbf{d f})^{\#}:}$ & Signifikanz & $\underline{\mathbf{F}(\mathbf{d f})^{\#}:}$ & Signifikanz & $\underline{F(d f)})^{\#}:$ & $\underline{\text { Signifikanz }}$ \\
\hline Fehlersumme I & $7,863(1,48)$ & $.007 * *$ & $14,447(1,48)$ & $.001 * *$ & $0,092(1,48)$ & .762 \\
\hline Fehlersumme II & $10,024(1,48)$ & $.003 * *$ & $0,176(1,48)$ & .676 & $1,935(1,48)$ & .171 \\
\hline Zeit I & $14,987(1,48)$ & $.001 * *$ & $5,289(1,48)$ & $.026^{*}$ & $0,627(1,48)$ & .432 \\
\hline Zeit II & $7,553(1,48)$ & $.008 * *$ & $1,896(1,48)$ & .175 & $1,413(1,48)$ & .240 \\
\hline
\end{tabular}

${ }^{\#}$ Testverfahren und Prüfgröße = zweifaktorielle ANOVA, F-Wert, df = Freiheitsgrade; * = signifikant bei $\alpha<0.05, * *=$ signifikant bei $\alpha<0.01$. 
Tabelle 12: $\quad$ Ergebnisse der zweifaktoriellen, multivariaten ANOVAs mit den Faktoren „Gruppe“ \& „Testumwelt“ (Fortsetzung)

\begin{tabular}{|c|c|c|c|c|c|c|}
\hline \multirow[t]{2}{*}{ Indikator: } & \multicolumn{2}{|c|}{$\frac{\frac{\text { Haupteffekt }}{{ }_{2} \text { Gruppe }^{66}}}{(\mathrm{n}=25)}$} & \multicolumn{2}{|c|}{$\frac{\frac{\text { Haupteffekt }}{\text { Testumwelt }^{66}}}{(\mathrm{n}=25)}$} & \multicolumn{2}{|c|}{$\frac{\frac{\underline{\text { Interaktion }}}{\text {,Gruppe - Testumwelt }}}{(n=25)}$} \\
\hline & $\underline{\mathbf{F}(\mathbf{d f})^{*}:}$ & Signifikanz & $\underline{\mathbf{F}(\mathbf{d f})^{\#}:}$ & Signifikanz & $\underline{\mathbf{F}(\mathbf{d f})^{\#}:}$ & $\underline{\text { Signifikanz }}$ \\
\hline Lernkurve I & $5,680(1,48)$ & $.021 *$ & $2,468(1,48)$ & .123 & $0,005(1,48)$ & .943 \\
\hline Lernkurve II & $2,687(1,48)$ & .108 & $2,442(1,48)$ & .125 & $0,595(1,48)$ & .444 \\
\hline
\end{tabular}

${ }^{\#}$ Testverfahren und Prüfgröße $=$ zweifaktorielle ANOVA, F-Wert, $\mathrm{df}=$ Freiheitsgrade; $*$ = signifikant bei $\alpha<0.05, * *=$ signifikant bei $\alpha<0.01$.

Aus Tabelle 12 wird ersichtlich, dass sich über fast alle Indikatoren ein signifikanter Hautpeffekt „Gruppe“ zeigte. Schizophrene Probanden wiesen im Vergleich zu gesunden Kontrollpersonen sowohl im ego-, als auch im allozentrischen Raum signifikant schwächere Navigationsleistungen mit den Indikatoren „Fehlersumme I“ (F = 7,863; p = .007), „Fehlersumme II“ (F = 10,024, p < .003), ,Zeit I“ (F = 14,987, p < .001), ,Zeit II“ $(\mathrm{F}=7,553, \mathrm{p}=.008)$ sowie „Lernkurve I“ $(\mathrm{F}=5,680, \mathrm{p}=.021)$ auf. Die deskriptiven Ergebnisse einer deutlich überlegenen ego- und allozentrischen Navigationsleistung in der gesunden Kontrollgruppe ließen sich somit durch die inferenzstatistische Analyse weitestgehend untermauern.

Darüber hinaus zeigte sich anhand der Indikatoren „Fehlersumme I“ $(F=14,447, \mathrm{p}<.001)$ und ,Zeit I“ (F = 5,289, p = .026) ein signifikanter Haupteffekt „Testumwelt“. Sowohl schizophrene, als auch gesunde Testpersonen wiesen mit diesen Indikatoren eine deutlich bessere Leistung im allozentrischen als im egozentrischen Raum auf.

Es ließen sich keinerlei signifikante Wechselwirkungen zwischen den Faktoren „Gruppe“ und ,Testumwelt" beobachten.

\section{Rekognitions- und Nachbefragungsbogen}

Ergänzend zur Navigationsleistung der Probanden wurde innerhalb des Rekognitions- und des Nachbefragungsbogens erfasst, ob die Probanden das durchlaufene Wegenetz wiedererkennen konnten, als wie schwierig sie die jeweilige Navigationsaufgabe empfunden hatten und welche Strategie sie zum Wiederfinden des Zielpunktes verwandten. Mit Hilfe von nonparametrischen Chi-Quadrat- sowie Mann-Whitney-U-Tests wurde geprüft, ob sich die Probandengruppen in diesen Maßen unterschieden. 
Tabelle 13: $\quad$ Ergebnisse des Rekognitions- \& des Nachbefragungsbogens

\begin{tabular}{|c|c|c|c|c|c|c|}
\hline $\begin{array}{l}\text { Gruppe: } \\
\text { Testvariable: } \\
\end{array}$ & \multicolumn{2}{|c|}{$\frac{\frac{\text { Schizophrene }}{\text { Patientengruppe }}}{(n=25)}$} & \multicolumn{2}{|c|}{$\frac{\begin{array}{c}\text { Gesunde } \\
\text { Kontrollgruppe }\end{array}}{(\mathbf{n}=25)}$} & \multicolumn{2}{|c|}{$\frac{\text { Testverfahren und }}{\text { Prüfgröß }^{\#}}$} \\
\hline $\begin{array}{l}\text { Rekognition ,Labyrinth“"? } \\
\text { 1) ja } \\
\text { 2) nein } \\
\end{array}$ & $\begin{array}{c}\frac{\text { Häufigkeit }}{12} \\
13 \\
\end{array}$ & $\begin{array}{l}\text { Prozent } \\
48 \% \\
52 \% \\
\end{array}$ & $\begin{array}{c}\frac{\text { Häufigkeit }}{17} \\
8 \\
\end{array}$ & $\begin{array}{c}\text { Prozent } \\
68 \% \\
32 \% \\
\end{array}$ & $\frac{\chi 2 \text {-Wert }}{2,053}$ & $\frac{\text { Signifikanz }}{\mathrm{p}=.152}$ \\
\hline $\begin{array}{l}\text { Rekognition ,Park }{ }^{66} \text { ? } \\
\text { 1) ja } \\
\text { 2) nein } \\
\end{array}$ & $\begin{array}{c}\text { Häufigkeit } \\
17 \\
6 \\
\end{array}$ & $\begin{array}{c}\text { Prozent } \\
68 \% \\
24 \% \\
\end{array}$ & $\begin{array}{c}\frac{\text { Häufigkeit }}{20} \\
5 \\
\end{array}$ & $\begin{array}{l}\frac{\text { Prozent }}{80 \%} \\
20 \% \\
\end{array}$ & $\frac{\chi 2 \text {-Wert }}{0,251}$ & $\frac{\text { Signifikanz }}{\mathrm{p}=.616}$ \\
\hline Schwierigkeit „Labyrinth“» & $\frac{\mathrm{MW}}{2,78}$ & $1, \frac{\mathrm{s}}{24}$ & $\frac{\mathrm{MW}}{3,36}$ & $\stackrel{\underline{\mathrm{s}}}{1,19}$ & $\frac{z-\text { Wert }}{-1,592}$ & $\frac{\text { Signifikanz }}{\mathrm{p}=.111}$ \\
\hline Schwierigkeit ,Park“» & $\frac{\mathrm{MW}}{3,48}$ & $\stackrel{\underline{\mathrm{s}}}{1,08}$ & $\frac{\mathrm{MW}}{4,52}$ & $0, \underline{\underline{s}}$ & $\frac{\mathrm{z} \text {-Wert }}{-3,361}$ & $\frac{\text { Signifikanz }}{p=.001^{* *}}$ \\
\hline $\begin{array}{ll}\text { Strategie „Labyrinth“ } \\
\text { 1) } & \text { verbal } \\
\text { 2) } & \text { visuell-räumlich } \\
\text { 3) } & \text { verbal und visuell } \\
\text { 4) } & \text { etwas anderes } \\
\end{array}$ & $\begin{array}{c}\text { Häufigkeit } \\
13 \\
5 \\
3 \\
2 \\
\end{array}$ & $\begin{array}{c}\text { Prozent } \\
52 \% \\
20 \% \\
12 \% \\
8 \% \\
\end{array}$ & $\begin{array}{c}\text { Häufigkeit } \\
14 \\
8 \\
1 \\
2 \\
\end{array}$ & $\begin{array}{c}\text { Prozent } \\
56 \% \\
32 \% \\
4 \% \\
8 \% \\
\end{array}$ & $\frac{\chi 2 \text {-Wert }}{1,649}$ & $\frac{\text { Signifikanz }}{\mathrm{p}=.648}$ \\
\hline \begin{tabular}{ll}
\multicolumn{2}{c}{ Strategie „Park“ } \\
5) & verbal \\
6) visuell-räumlich \\
7) & verbal und visuell \\
8) & etwas anderes
\end{tabular} & $\begin{array}{c}\text { Häufigkeit } \\
4 \\
16 \\
4 \\
0\end{array}$ & $\begin{array}{l}\text { Prozent } \\
16 \% \\
64 \% \\
16 \% \\
0 \%\end{array}$ & $\begin{array}{c}\text { Häufigkeit } \\
1 \\
21 \\
3 \\
0\end{array}$ & $\begin{array}{l}\text { Prozent } \\
4 \% \\
84 \% \\
12 \% \\
0 \%\end{array}$ & $\frac{\chi 2 \text {-Wert }}{3,249}$ & $\frac{\text { Signifikanz }}{\mathrm{p}=.355}$ \\
\hline
\end{tabular}

MW = Mittelwert; $\mathbf{s}=$ Standardabweichung; ${ }^{\#}$ Testverfahren und Prüfgröße $=$ Mann-Whitney-U-Test, z-Wert und Chi-Quadrat-Test, $\chi 2 ;{ }^{\S}$ Schwierigkeitsrating: $1=$ sehr schwer, $5=$ sehr leicht; $* *=$ signifikant bei $\alpha<0.01$.

Auf deskriptiver Ebene zeigte sich auch innerhalb des Rekognitions- und des Nachbefragungsbogens eine konsistent schwächere Leistung bei der schizophrenen Probandengruppe: Sie erkannten die durchlaufenen Wegenetze seltener wieder (12 vs. 17 Rekognitionen im Labyrinth, 17 vs. 20 Rekognitionen im Park) und beurteilen die Aufgabenschwierigkeit insgesamt höher als gesunde Testpersonen (MW = 2,78 bzw. 3,48 in der Patientengruppe vs. MW = 3,36 bzw. 4,52 in der Kontrollgruppe).

Inferenzstatistisch zeigte sich bei der schizophrenen Probandengruppe eine deutlich höhere Beurteilung der Aufgabenschwierigkeit im virtuellen Park $(z=-3,361 ; p=.001)$.

Es fanden sich weder im Labyrinth, noch im Park Unterschiede in der verwandten (verbalen oder visuellen) Navigationsstrategie zwischen Patienten und Kontrollpersonen.

22 Anmerkung: Die Kategorien „,visuell““ und „räumlich“ des Nachbefragungsbogens wurden zu einer gemeinsamen Kategorie, ,visuell-räumlich“ zusammengefasst. 


\subsubsection{Hirnvolumetrie: Parietale Cortexvolumina}

\section{Deskription}

In der vorliegenden Arbeit lagen die hirnanatomischen Datensätze von 39 schizophrenen Probanden und 39 Kontrollpersonen vor. Tabelle 14 gibt die Kennwerte der parietalen Volumetrierung vergleichend wieder.

Tabelle 14: $\quad$ Parietale Cortexvolumina der Patienten- \& der Kontrollgruppe

\begin{tabular}{|c|c|c|c|c|c|c|c|c|c|}
\hline $\begin{array}{l}\text { Gruppe: } \\
\text { Cortexareal: }\end{array}$ & \multicolumn{2}{|c|}{$\frac{\text { Patienten- }}{\frac{\text { gruppe }}{(n=39)}}$} & \multicolumn{2}{|c|}{$\frac{\text { Kontroll- }}{\frac{\text { gruppe }}{(n=39)}}$} & \multirow[t]{2}{*}{$\begin{array}{r}\text { Gruppe: } \\
\text { Cortexareal } \\
\end{array}$} & \multicolumn{2}{|c|}{$\frac{\text { Patienten- }}{\frac{\text { gruppe }}{(n=39)}}$} & \multicolumn{2}{|c|}{$\frac{\text { Kontroll- }}{\frac{\text { gruppe }}{(n=39)}}$} \\
\hline & $\underline{\text { MW }}$ & $\underline{\mathbf{S}}$ & $\underline{\text { MW }}$ & $\underline{\mathbf{S}}$ & & $\underline{\text { MW }}$ & $\underline{\mathbf{S}}$ & $\underline{\text { MW }}$ & $\underline{\mathbf{S}}$ \\
\hline $\begin{array}{c}\text { Parietallappen } \\
\text { rechts }^{+}\end{array}$ & 71,91 & 12,83 & 78,14 & 13,50 & $\begin{array}{l}\text { Parietallappen } \\
\operatorname{links}^{+}\end{array}$ & 73,76 & 13,58 & 79,66 & 11,27 \\
\hline $\begin{array}{c}\text { Superiorer PL } \\
\text { Rechts }^{+}\end{array}$ & 49,22 & 9,69 & 54,16 & 10,35 & $\begin{array}{c}\text { Superiorer PL } \\
\operatorname{links}^{+}\end{array}$ & 50,36 & 9,73 & 55,39 & 9,21 \\
\hline $\begin{array}{c}\text { Inferiorer } \mathbf{P L} \\
\text { rechts }^{+}\end{array}$ & 22,69 & 5,43 & 23,98 & 5,83 & $\underset{\operatorname{links}^{+}}{\text {Inferiorer }} \mathbf{P L}$ & 23,39 & 6,55 & 24,28 & 5,61 \\
\hline
\end{tabular}

MW = Mittelwert; $\mathbf{s}=$ Standardabweichung; ${ }^{+}=$Angabe in $\mathbf{m l}$.

Wie aus Tabelle 14 ersichtlich, waren auf deskriptiver Ebene alle parietalen Volumina in der schizophrenen Patientengruppe kleiner als in der gesunden Vergleichsgruppe.

\section{Inferenzstatistik}

Um zu überprüfen, ob sich die Volumina des parietalen Cortex oder seiner Subareale zwischen der Patienten- und der Kontrollgruppe auf inferenzstatistischer Ebene voneinander unterscheiden, wurden zweifaktorielle, multivariate Kovarianzanalysen (analysis of covariance, ANCOVAs) mit den abhängigen Variablen „Parietallappen“, „superiorer Parietallappen“ sowie „inferiorer Parietallappen“ und den jeweils zweistufigen Faktoren „Gruppe“ (schizophrene vs. gesunde Testpersonen) und „Hemisphäre“ (rechts vs. links) durchgeführt. Dabei wurde das globale Cortexvolumen als Kovariate einbezogen (vgl. Barta et al., 1990; Schlaepfer et al., 1994; Shenton et al., 2001; Cannon et al., 2002). Tabelle 15 gibt die relevanten varianzanalytischen Kennwerte wieder: 
Tabelle 15: $\quad$ Ergebnisse der zweifaktoriellen, multivariaten ANCOVAs mit den Faktoren „Gruppe“ \& „Hemisphäre“

\begin{tabular}{|c|c|c|c|c|c|c|}
\hline \multirow[t]{2}{*}{ Cortexareal: } & \multicolumn{2}{|c|}{$\frac{\text { Haupteffekt }}{\frac{{ }_{2} \text { Gruppe }^{66}}{(n=39)}}$} & \multicolumn{2}{|c|}{$\frac{\frac{\text { Haupteffekt }}{\text { Hemisphäre }}}{(n=39)}$} & \multicolumn{2}{|c|}{$\frac{\text { Interaktion }}{\text { פGruppe - Hemisphäre }}$} \\
\hline & $\underline{\mathbf{F}(\mathbf{d f})}{ }^{\#}:$ & $\underline{\text { Signifikanz }}$ & $\underline{\mathbf{F}(\mathbf{d f})}{ }^{\#}:$ & $\underline{\text { Signifikanz }}$ & $\underline{\mathbf{F}(\mathbf{d f})^{\#}} \underline{:}$ & $\underline{\text { Signifikanz }}$ \\
\hline Parietallappen & 4,534 (1,75) & $.037 *$ & $0,032(1,75)$ & .859 & $0,035(1,75)$ & .853 \\
\hline Superiorer PL & $5,477(1,75)$ & $.022 *$ & $1,466(1,75)$ & .230 & $0,025(1,75)$ & .874 \\
\hline Inferiorer PL & $0,412(1,75)$ & .523 & $3,131(1,75)$ & .081 & $0,234(1,75)$ & .630 \\
\hline
\end{tabular}

\# Testverfahren und Prüfgröße $=$ zweifaktorielle ANCOVA, F-Wert, df = Freiheitsgrade; * = signifikant bei $\alpha<0.05$.

Auf inferenzstatistischer Ebene ließ sich ein signifikanter Haupteffekt „Gruppe“ für den globalen Parietallappen sowie für sein superiores Subareal zeigen $(F=4,534, p=.037$ und $\mathrm{F}=5,477, \mathrm{p}=.022)$. In diesen beiden Arealen wiesen schizophrene Probanden geringere Volumninae auf als die gesunde Vergleichsgruppe.

Effekte für den Faktor „Hemisphäre“ und Interaktionseffekte zwischen den Faktoren „Gruppe“ und „Hemisphäre“ konnten mit dem vorliegenden Datenmaterial nicht festgestellt werden.

\subsubsection{Zusammenhänge Neuropsychologie - Hirnvolumetrie}

Um die vermuteten Zusammenhänge zwischen der visuell-räumlichen Navigationsleistung und parietalen Cortexvolumina untersuchen $\mathrm{zu}$ können, wurden die verschiedenen Indikatoren der Navigationsleistung mit den verschiedenen parietalen Cortexvolumina korreliert. Die Analysen erfolgten separat für die Patienten- und für die Kontrollgruppe, wobei 21 beziehungsweise 25 Datensätze eingingen.

Berechnungen mit den Indikatoren „Fehlersumme I und II“ erfolgten nonparametrisch, mit Hilfe von Spearman-Rangkorrelationen, Berechnungen mit den Indikatoren „Zeit“" und „Lerngewinn“ parametrisch, mit Hilfe von Pearson-Korrelationen. 


\subsubsection{Kontrollgruppe}

In der Kontrollgruppe zeigten sich keine signifikanten Korrelationen zwischen parietalen Hirnvolumina und der Navigationsleistung im ego- oder allozentrischen Raum. Eine Auflistung der einzelnen Kennwerte findet sich unter 7.4.1..

\subsubsection{Schizophrene Probandengruppe}

Die Tabellen 16 und 17 geben die Zusammenhänge zwischen den parietalen Hirnvolumina und der egozentrischen sowie allozentrischen Navigationsleistung für die schizophrene Probandengruppe wieder.

Tabelle 16: Zusammenhang zwischen der Navigationsleistung im egozentrischen Raum \& parietalen Hirnvolumina (Patientengruppe, $n=21$ )

\begin{tabular}{|c|c|c|c|c|c|c|}
\hline $\begin{array}{c}\text { Navigations- } \\
\text { leistung: } \\
\text { Cortexareal: }\end{array}$ & $\begin{array}{l}\text { Fehler- } \\
\text { summe I } \\
\underline{1}\end{array}$ & $\begin{array}{c}\text { Fehler- } \\
\text { summe II } \\
\end{array}$ & $\underline{\text { Zeit } \mathbf{I}^{2}}$ & $\underline{\text { Zeit } \text { II }^{2}}$ & $\stackrel{\text { Lern- }}{\text { gewinn } I^{2}}$ & $\stackrel{\text { Lern- }}{\text { gewinn II' }}^{2}$ \\
\hline & $\underline{\mathbf{r}}_{\mathrm{s}}:$ & $\underline{\mathbf{r}}_{\underline{s}}$ & $\underline{r}:$ & $\underline{\text { r: }}$ & $\underline{r}:$ & $\underline{\mathbf{r}}:$ \\
\hline $\begin{array}{l}\text { Parietallappen } \\
\text { rechts }\end{array}$ & -.147 & -.162 & -.234 & -.109 & .391 & $.487 *$ \\
\hline $\begin{array}{c}\text { Parietallappen } \\
\text { links }\end{array}$ & -.298 & -.203 & -.272 & -.094 & .368 & $.507 *$ \\
\hline $\begin{array}{c}\text { Superiorer PL } \\
\text { rechts }\end{array}$ & -.128 & -.155 & -.221 & -.106 & .311 & $.438 *$ \\
\hline $\begin{array}{c}\text { Superiorer PL } \\
\text { links }\end{array}$ & -.145 & -.021 & -.142 & .032 & .207 & .415 \\
\hline $\begin{array}{c}\text { Inferiorer PL } \\
\text { rechts }\end{array}$ & -.159 & -.176 & -.150 & -.061 & .397 & .366 \\
\hline $\begin{array}{c}\text { Inferiorer PL } \\
\text { links }\end{array}$ & -.240 & -.175 & -.346 & 244.-. & $.447 *$ & .420 \\
\hline
\end{tabular}

Testverfahren und Prüfgröße: ${ }^{1}$ Spearman Rangkorrelation, $\mathrm{r}_{\mathrm{s}}$; ${ }^{2}$ Pearson-Korrelation, $\mathrm{r}$; ${ }^{*}=$ signifikant bei $\alpha<0.05$.

Im egozentrischen Raum ließen sich signifikante Korrelationen zwischen dem Parietallappenvolumen der rechten und der linken Hemisphäre und dem „Lerngewinn II“ (r $=.487 ; \mathrm{p}=.025$ und $\mathrm{r}=.507 ; \mathrm{p}=.019)$, zwischen dem rechten superioren Parietallappen und dem „Lerngewinn II“ $(r=.438 ; \mathrm{p}=.047)$ sowie zwischen dem linken inferioren Parietallappen und dem „Lerngewinn $\mathrm{I}^{“}(\mathrm{r}=.447 ; \mathrm{p}=.042)$ nachweisen. 
Tabelle 17: Zusammenhang zwischen der Navigationsleistung im allozentrischen Raum \& parietalen Hirnvolumina (Patientengruppe; $n=21$ )

\begin{tabular}{|c|c|c|c|c|c|c|}
\hline $\begin{array}{c}\text { Navigations- } \\
\text { leistung: } \\
\text { Cortexareal: }\end{array}$ & $\begin{array}{c}\text { Fehler- } \\
\text { summe I } 1 \\
\underline{\underline{y}}\end{array}$ & $\begin{array}{l}\underline{\text { Fehler- }}^{\text {summe II }} \\
\underline{\text { sum }^{2}}\end{array}$ & $\underline{\text { Zeit } \mathbf{I}^{2}}$ & $\underline{\text { Zeit II }^{2}}$ & $\frac{\text { Lern- }}{{\text { gewinn } \mathbf{I}^{2}}^{2}}$ & $\frac{\text { Lern- }}{\text { gewinn II }}$ \\
\hline & $\underline{\mathbf{r}}_{\underline{s}}:$ & $\underline{\mathbf{r}}_{\underline{s}}$ & $\underline{\mathbf{r}}:$ & $\underline{\mathbf{r}}:$ & $\underline{\mathbf{r}}:$ & $\underline{\mathbf{r}}:$ \\
\hline $\begin{array}{c}\text { Parietallappen } \\
\text { rechts }\end{array}$ & $-.453^{*}$ & -.151 & -.366 & .148 & .399 & $.584 * *$ \\
\hline $\begin{array}{c}\text { Parietallappen } \\
\text { links }\end{array}$ & -.338 & -.100 & -.253 & -.088 & .245 & .403 \\
\hline $\begin{array}{c}\text { Superiorer PL } \\
\text { rechts }\end{array}$ & -.373 & -.034 & -.402 & -.171 & $.449 *$ & $.623 * *$ \\
\hline $\begin{array}{c}\text { Superiorer PL } \\
\text { links }\end{array}$ & -.239 & -.113 & -.237 & -.118 & .215 & .361 \\
\hline $\begin{array}{l}\text { Inferiorer PL } \\
\text { rechts }\end{array}$ & -.290 & -.113 & -.090 & -.014 & .070 & .191 \\
\hline $\begin{array}{l}\text { Inferiorer PL } \\
\text { links }\end{array}$ & -.255 & -.042 & .-165 & -.003 & .182 & .285 \\
\hline
\end{tabular}

Testverfahren und Prüfgröße: ${ }^{1}$ Spearman Rangkorrelation, $\mathrm{r}_{\mathrm{s}}$; ${ }^{2}$ Pearson-Korrelation, $\mathrm{r}$; ${ }^{*}=$ signifikant bei $\alpha<0.05 ; * *=$ signifikant bei $\alpha<0.01$.

Im Vergleich zur egozentrischen Navigationsleistung ließen sich zum Teil noch deutlichere Effekte in der allozentrischen Navigationsleistung beobachten: Das Volumen des rechten Parietallappens korrelierte signifikant mit den Indikatoren „Fehlersumme I“ $\left(r_{s}=-.453 ; p=\right.$ .039) und „Lerngewinn II“ $(\mathrm{r}=.584 ; \mathrm{p}=.005)$, das Volumen des rechten superioren Parietallappens mit den Indikatoren „Lerngewinn I“ $(r=.449 ; p=.041)$ und „Lerngewinn II“" $(r=.623 ; p=.003)$. 


\subsection{Explorative und validierende Hypothesen}

\subsubsection{Hippocampale Areale}

\section{Hippocampusvolumina}

Analog zu parietalen Cortexarealen wurden auch die Volumina der hippocampalen Cortexareale von 39 schizophrenen Probanden und 39 gesunden Kontrollpersonen mit Hilfe einer zweifaktoriellen ANCOVA verglichen, wobei das globale Cortexvolumen erneut als Kovariate einbezogen wurde. Die Tabellen 18 und 19 geben die deskriptiven und inferenzstatistischen Befunde der hippocampalen Analysen wieder.

Tabelle 18: $\quad$ Hippocampale Cortexvolumina in der Patienten- \& der Kontrollgruppe

\begin{tabular}{|c|c|c|c|c|}
\hline Gruppe: & \multicolumn{2}{|c|}{$\frac{\frac{\text { Schizophrene }}{\text { Patientengruppe }}}{(n=39)}$} & \multicolumn{2}{|c|}{$\frac{\begin{array}{c}\text { Gesunde } \\
\text { Kontrollgruppe }\end{array}}{(n=39)}$} \\
\hline & MW & $\underline{\mathrm{S}}$ & MW & $\underline{\mathrm{S}}$ \\
\hline Hippocampus rechts ${ }^{+}$ & 3108,69 & 432,33 & 3512,28 & 495,95 \\
\hline Hippocampus links ${ }^{+}$ & 3043,28 & 352,25 & 3398,92 & 540,70 \\
\hline
\end{tabular}

MW = Mittelwert; $s=$ Standardabweichung; ${ }^{+}$Angabe in Voxeln.

Tabelle 19: $\quad$ Ergebnisse der zweifaktoriellen, multivariaten ANCOVA mit den Faktoren „Gruppe“ \& „Hemisphäre“

\begin{tabular}{|c|c|c|c|c|c|c|}
\hline \multirow[t]{2}{*}{ Cortexareal: } & \multicolumn{2}{|c|}{$\frac{\frac{\text { Haupteffekt }}{\text { gruppe }^{66}}}{(\mathrm{n}=39)}$} & \multicolumn{2}{|c|}{$\frac{\frac{\text { Haupteffekt }}{\text { Hemisphäre }}}{(n=39)}$} & \multicolumn{2}{|c|}{$\frac{\text { פgruppe - Hemisphäre }}{\text { „66 }}$} \\
\hline & ${\underline{\mathbf{F}}(\mathbf{d f})^{\#}:}^{\#}$ & Signifikanz & ${\underline{\mathbf{F}}(\mathbf{d f})^{\#}}^{\#}$ & Signifikanz & $\underline{\mathbf{F}(\mathbf{d f})}{ }^{\#}:$ & $\underline{\text { Signifikanz }}$ \\
\hline Hippocampus & $16,781(1,75)$ & $<.001 * *$ & $0,111(1,75)$ & .740 & $0,396(1,75)$ & .531 \\
\hline
\end{tabular}

\# Testverfahren und Prüfgröße $=$ zweifaktorielle ANCOVA, F-Wert, $\mathrm{df}=$ Freiheitsgrade; ${ }^{* *}=$ signifikant bei $\alpha<0.01$.

Wie aus dem signifikanten Haupteffekt „Gruppe“ und den deskriptiven Statistiken ersichtlich (MW = 3108,69 und 3043,28 vs. $\mathrm{MW}=3512,28$ und 3398,92), wiesen schizophrene Probanden im Vergleich $\mathrm{zu}$ gesunden Testpersonen deutlich geringere hippocampale Volumina auf $(\mathrm{F}=16,781, \mathrm{p}<.001)$.

Der Haupteffekt „Hemisphäre“ und die Interaktion zwischen den Faktoren „Gruppe“ und „Hemisphäre“ waren nicht signifikant. 


\section{Zusammenhänge zwischen der Navigationsleistung und den Hippocampusvolumina}

Analog zu dem unter 4.1.3. beschriebenen Vorgehen zu parietalen Arealen, wurde auch die Überprüfung korrelativer Zusammenhänge zwischen ego- und allozentrischen Navigationsleistungen und hippocampalen Arealen mit Hilfe von non-parametrischen Spearman-Rangkorrelationen und parametrischen Pearson-Korrelationen durchgeführt. Dabei erfolgten die Analysen erneut separat für die Patienten- und die Kontrollgruppe.

Weder in der Kontrollgruppe, noch in der Patientengruppe ließen sich signifikante Zusammenhänge zwischen hippocampalen Hirnvolumina und der Navigationsleistung im ego- oder allozentrischen Raum beobachten. Die statistischen Kennwerte der korrelativen Analysen sind unter 7.4.2. zu finden.

\subsubsection{Schizophrene Subgruppen}

\subsubsection{Visuell-räumliche Navigationsleistung}

Die Tabellen 20 und 21 geben die Navigationsleistung der paranoiden und desorganisierten schizophrenen Subgruppen sowie der Kontrollgruppe im ego- und allozentrischen Raum wieder. Die undifferenziert-schizophrene Subgruppe wurde aufgrund ihrer geringen Stichprobengröße von nur drei Probanden von der Subtypenanalyse ausgeschlossen.

Tabelle 20: $\quad$ Navigationsleistung der schizophrenen Subgruppen \& der gesunden Kontrollgruppe im egozentrischen Raum

\begin{tabular}{|c|c|c|c|c|c|c|}
\hline \multirow[t]{2}{*}{$\begin{array}{l}\text { Gruppe: } \\
\text { Indikator: }\end{array}$} & \multicolumn{2}{|c|}{$\frac{\frac{\text { Paranoide }}{\text { Subgruppe }}}{(\mathrm{n}=16)}$} & \multicolumn{2}{|c|}{$\frac{\frac{\text { Desorganisierte }}{\text { Subgruppe }}}{(\mathrm{n}=5)}$} & \multicolumn{2}{|c|}{$\frac{\begin{array}{c}\text { Gesunde } \\
\text { Kontrollgruppe }\end{array}}{(n=25)}$} \\
\hline & $\underline{\text { MW }}$ & $\underline{\mathbf{s}}$ & $\underline{\text { MW }}$ & $\underline{\mathbf{s}}$ & $\underline{\text { MW }}$ & $\underline{\mathbf{s}}$ \\
\hline Fehlersumme I & 13,87 & 12,53 & 11,80 & 10,73 & 5,84 & 10,43 \\
\hline Fehlersumme II & 17,75 & 10,93 & 18,80 & 9,14 & 13,08 & 10,64 \\
\hline Zeit I & 197,14 & 92,49 & 200,90 & 86,19 & 130,36 & 60,84 \\
\hline Zeit II & 863,35 & 355,52 & 906,18 & 342,07 & 734,86 & 321,54 \\
\hline Lernkurve I & $-13,57$ & 149,30 & 25,83 & 154,13 & 74,27 & 71,24 \\
\hline Lernkurve II & 23,94 & 179,21 & 145,90 & 299,43 & 218,31 & 257,01 \\
\hline
\end{tabular}

MW = Mittelwert; $\mathbf{s}=$ Standardabweichung. 
Tabelle 21: $\quad$ Navigationsleistung der schizophrenen Subgruppen \& der gesunden Kontrollgruppe im allozentrischen Raum

\begin{tabular}{|c|c|c|c|c|c|c|}
\hline \multirow[t]{2}{*}{$\begin{array}{l}\text { Gruppe: } \\
\text { Indikator: }\end{array}$} & \multicolumn{2}{|c|}{$\frac{\frac{\text { Paranoide }}{\text { Subgruppe }}}{(\mathrm{n}=16)}$} & \multicolumn{2}{|c|}{$\frac{\frac{\text { Desorganisierte }}{\text { Subgruppe }}}{(\mathbf{n}=5)}$} & \multicolumn{2}{|c|}{$\frac{\begin{array}{c}\text { Gesunde } \\
\text { Kontrollgruppe }\end{array}}{(n=25)}$} \\
\hline & $\underline{\text { MW }}$ & $\underline{\mathbf{s}}$ & $\underline{\text { MW }}$ & $\underline{\mathbf{s}}$ & $\underline{\text { MW }}$ & $\underline{\mathbf{s}}$ \\
\hline Fehlersumme I & 5,56 & 6,55 & 6,80 & 7,01 & 0,32 & 0,63 \\
\hline Fehlersumme II & 20,75 & 14,94 & 22,80 & 16,39 & 9,20 & 5,66 \\
\hline Zeit I & 167,95 & 95,87 & 213,80 & 106,56 & 92,53 & 11,37 \\
\hline Zeit II & 866,48 & 400,80 & 957,58 & 358,65 & 569,77 & 222,01 \\
\hline Lernkurve I & 44,62 & 118,39 & $-38,14$ & 77,34 & 100,55 & 70,41 \\
\hline Lernkurve II & 190,83 & 270,47 & 271,49 & 354,46 & 256,39 & 196,65 \\
\hline
\end{tabular}

MW = Mittelwert; $\mathrm{s}=$ Standardabweichung.

Zum inferenzstatistischen Vergleich der Subgruppen wurde - analog zu 4.1.1. - ein zweifaktorieller, nonparametrischer Split-Plot-Test mit den Faktoren Gruppe (hier: „paranoid“ vs. „desorganisiert“ vs. „gesund“) und Testumwelt (,VR-Labyrinth“ vs. „,VRPark“) durchgeführt. Im Falle signifikanter Befunde erfolgte eine differenziertere post-hoc Analyse mit Hilfe von nonparametrischen ANOVA-type-Tests. Die Ergebnisse sind in Tabelle 22 dargestellt. $^{23}$

\footnotetext{
23 Aufgrund der kleinen Zellenbesetzungen wurden ausschließlich nonparametrische Split-plot-Tests mit anschließenden ANOVA-type-Tests durchgeführt. Bei diesen Tests handelt es sich um die nonparametrischen Entsprechungen von zweifaktoriellen Overall-ANOVAS sowie einfaktoriellen Post-hoc ANOVAS. Für detailliertere Informationen siehe http://www.ams.med.uni-goettingen.de/Projekte/LD/Makros_LD.html.
} 
Tabelle 22: $\quad$ Ergebnisse der zweifaktoriellen, multivariaten Subtypenanalysen mit den Faktoren "Subgruppe“ \& „Testumwelt“

\begin{tabular}{|c|c|c|c|c|c|c|}
\hline \multirow[t]{2}{*}{ Indikator: } & \multicolumn{2}{|c|}{$\frac{\text { Haupteffekt }}{{ }_{2} \text { Subgruppe }}$} & \multicolumn{2}{|c|}{$\frac{\text { Haupteffekt }}{\text {,Testumwelt }}$} & \multicolumn{2}{|c|}{$\frac{\underline{\text { Interaktion }}}{\text {,.Subgruppe }}$} \\
\hline & $\chi^{2}(\mathbf{d f})^{\#}:$ & Signifikanz & $\chi^{2}(\mathbf{d f})^{\#}:$ & Signifikanz & $\chi^{2}(\mathrm{df})^{\#}:$ & $\underline{\text { Signifikanz }}$ \\
\hline Fehlersumme I & $5,020(1)$ & $.011 *$ & $10,391(1)$ & $.001 * *$ & $0,001(1)$ & .999 \\
\hline Fehlersumme II & 3,947 (1) & $.027 *$ & $0,016(1)$ & .898 & $0,234(1)$ & .713 \\
\hline Zeit I & $5,104(1)$ & $.011 *$ & $4,260(1)$ & $.039 *$ & $0,234(1)$ & .731 \\
\hline Zeit II & $5,936(1)$ & $.003 * *$ & $0,028(1)$ & .867 & $0,555(1)$ & .522 \\
\hline Lernkurve I & $1,912(1)$ & .158 & $0,021(1)$ & .885 & $3,954(1)$ & $.021 *$ \\
\hline Lernkurve II & $0,726(1)$ & .428 & $2,370(1)$ & .124 & $0,142(1)$ & .790 \\
\hline
\end{tabular}

\# Testverfahren und Prüfgröße $=$ zweifaktorielle, nonparametrische ANOVA, $\chi^{2}$-Wert, df $=$ Freiheitsgrade; * = signifikant bei $\alpha<0.05 ; * *=$ signifikant bei $\alpha<0.01$.

Mit dem vorliegenden Datenmaterial ließ sich anhand der Indikatoren „Fehlersumme I“ $\left(\chi^{2}\right.$ $=5,020, \mathrm{p}=.011)$, ,Fehlersumme II“ $\left(\chi^{2}=3,947, \mathrm{p}=.027\right)$, ,Zeit I“ $\left(\chi^{2}=5,104, \mathrm{p}=.011\right)$ und ,Zeit II“ $\left(\chi^{2}=5,936, \mathrm{p}=.003\right)$ ein signifikanter Haupteffekt „Subgruppe“ zeigen. Darüber hinaus konnte mit den Indikatoren „Fehlersumme I“ $\left(\chi^{2}=10,391, p=.001\right)$ und „Zeit I“ $\left(\chi^{2}=4,260, \mathrm{p}=.039\right)$ ein signifikanter Hautpeffekt „Testumwelt“ beobachtet werden. Mit dem Indikator „Lernkurve I“ zeigte sich zudem eine signifikante Wechselwirkung der Faktoren „Subgruppe“ und „Testumwelt“ $\left(\chi^{2}=3,954, \mathrm{p}=.021\right)$.

Die Befunde der post-hoc-Analysen bestätigen im Wesentlichen die Ergebnisse der Hauptanalyse (vgl. 4.1.1.): Gesunde Testpersonen wiesen mit den oben genannten Indikatoren eine bessere ego- und allozentrische Navigationsleistung auf als paranoide oder desorganisierte Patienten. Darüber hinaus zeigte sich, dass alle Subgruppen mit dem Indikator „Fehlersumme I“ bessere Navigationsleistungen im virtuellen Park als im virtuellen Labyrinth verzeichnen konnten. Derselbe Effekt zeigte sich für gesunde und paranoide Probanden mit dem Indikator „Zeit I“. Aufgrund der großen Datenmenge und der inhaltlichen Übereinstimmung mit der Hauptanalyse, werden die statistischen Kennwerte der Haupteffekte an dieser Stelle nicht aufgeführt; eine detaillierte Darstellung findet sich unter 7.4.3.. 
Im Hinblick auf die Subtypenanalyse erscheint vor allem der signifikante Interaktionseffekt wesentlich, der sich mit dem Indikator „Lernkurve I“ zwischen der paranoiden und der desorganisierten Subgruppe finden ließ $(\mathrm{F}=5,657, \mathrm{p}=.017$; vgl. 7.4.3.). Abbildung 11 veranschaulicht die gefundene Wechselwirkung graphisch:

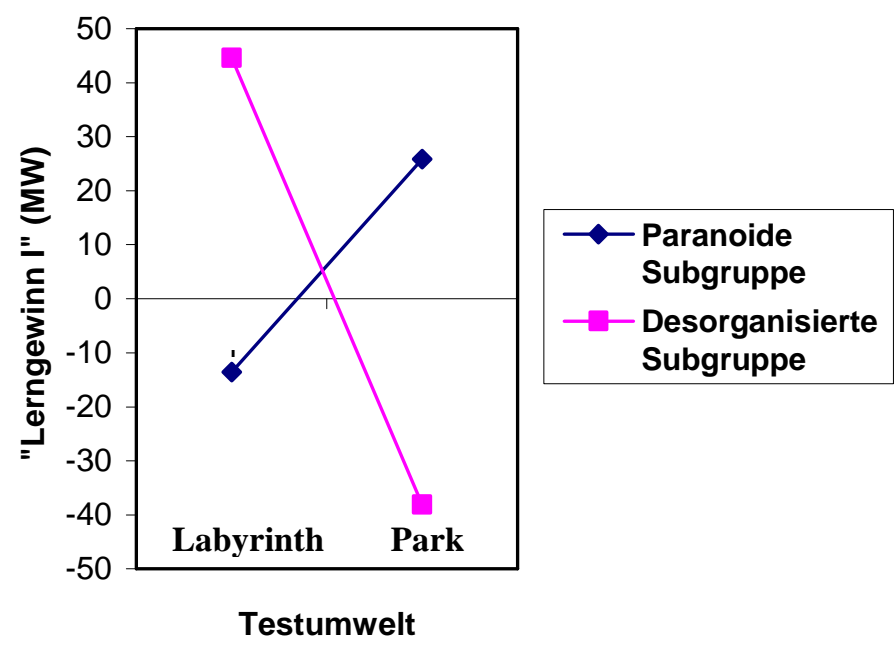

Abbildung 11: Interaktionseffekt zwischen der paranoiden \& der desorganisierten Subgruppe

Es zeigte sich, dass paranoid-schizophrene Testpersonen insbesondere Schwierigkeiten mit der egozentrischen Navigationsaufgabe (Labyrinth) hatten und eine schwächere Leistung aufwiesen als desorganisiert-schizophrene Probanden. Dagegen wiesen im allozentrischen Raum (Park) vor allem desorganisiert-schizophrene Probanden Navigationsdefizite auf; ihre Leistung war der von paranoid-schizophrenen Testpersonen unterlegen.

\subsubsection{Parietale Cortexvolumina}

Tabelle 23 gibt die parietalen Volumina der paranoiden, der desorganisierten und der undifferenzierten Subgruppe sowie der gesunden Kontrollgruppe vergleichend wieder. 
Tabelle 23: $\quad$ Parietale Cortexvolumina der schizophrenen Subgruppen \& der gesunden Kontrollgruppe

\begin{tabular}{|c|c|c|c|c|c|c|c|c|}
\hline \multirow[t]{2}{*}{$\begin{array}{r}\text { Gruppe: } \\
\text { Cortexareal: } \\
\end{array}$} & \multicolumn{2}{|c|}{$\frac{\frac{\text { Paranoide }}{\text { Subgruppe }}}{(\mathrm{n}=23)}$} & \multicolumn{2}{|c|}{$\frac{\text { Desorganisierte }}{\frac{\text { Subgruppe }}{(n=7)}}$} & \multicolumn{2}{|c|}{$\frac{\text { Undifferenzierte }}{\frac{\text { Subgruppe }}{(n=7)}}$} & \multicolumn{2}{|c|}{$\frac{\begin{array}{c}\text { Gesunde } \\
\text { Kontrollgruppe }\end{array}}{(n=39)}$} \\
\hline & MW & $\underline{\mathbf{s}}$ & MW & $\underline{\mathbf{s}}$ & MW & $\underline{\mathbf{s}}$ & MW & $\underline{\mathbf{s}}$ \\
\hline $\begin{array}{l}\text { Parietallappen } \\
\text { rechts }\end{array}$ & 69,83 & 11,88 & 82,67 & 16,81 & 69,53 & 7,25 & 78,14 & 13,50 \\
\hline $\begin{array}{c}\text { Parietallappen } \\
\text { links }\end{array}$ & 71,38 & 12,52 & 82,27 & 16,65 & 74,04 & 11,56 & 79,66 & 11,27 \\
\hline $\begin{array}{c}\text { Superiorer PL } \\
\text { rechts }\end{array}$ & 47,87 & 9,42 & 56,43 & 12,76 & 48,04 & 4,62 & 54,16 & 10,35 \\
\hline $\begin{array}{c}\text { Superiorer PL } \\
\text { links }\end{array}$ & 49,09 & 9,04 & 56,74 & 11,64 & 50,19 & 7,93 & 55,39 & 9,21 \\
\hline $\begin{array}{c}\text { Inferiorer PL } \\
\text { rechts }\end{array}$ & 21,95 & 5,49 & 26,24 & 6,40 & 21,49 & 3,37 & 23,98 & 5,83 \\
\hline $\begin{array}{c}\text { Inferiorer PL } \\
\text { links }\end{array}$ & 22,29 & 6,40 & 25,53 & 7,52 & 23,86 & 6,52 & 24,28 & 5,61 \\
\hline
\end{tabular}

MW = Mittelwert; $\mathrm{s}=$ Standardabweichung.

Beim Vergleich der parietalen Cortexareale zeigten sich auf deskriptiver Ebene durchgängig größere Volumina in der desorganisierten Subgruppe als in der paranoiden oder in der undifferenzierten Subgruppe.

Dieser Trend ließ sich auf inferenzstatistischer Ebene allerdings nicht bestätigen. Wie Tabelle 24 zeigt, konnten mit Hilfe einer nonparametrischen Kovarianzanalyse keine differentiellen hirnanatomischen Profile für die schizophrenen Subgruppen gefunden werden. Auch für den Haupteffekt „Hemisphäre“ sowie für den Interaktionseffekt „Subgruppe“ - „Hemisphäre“ konnten keine signifikanten Befunde nachgewiesen werden.

Tabelle 24: $\quad$ Ergebnisse der zweifaktoriellen, multivariaten Subtypenanalysen mit den Faktoren „Subgruppe“ \& „Hemisphäre“

\begin{tabular}{|c|c|c|c|c|c|c|}
\hline \multirow[t]{2}{*}{ Cortexareal: } & \multicolumn{2}{|c|}{$\frac{\text { Haupteffekt }}{\text { 2.Subgruppe }^{66}}$} & \multicolumn{2}{|c|}{${ }_{\text {„Hemisphäre }}^{\text {Haupteffekt }}$} & \multicolumn{2}{|c|}{$\begin{array}{l}\text { Interaktion } \\
\text { 2.Subgruppe }- \\
\underline{\text { Hemisphäre }}\end{array}$} \\
\hline & $\underline{t}(\mathbf{d f})^{\#}:$ & Signifikanz & $\underline{\mathbf{t}(\mathbf{d f})^{\#}:}$ & Signifikanz & $\underline{\mathbf{t}(\mathbf{d f})^{\#}:}$ & $\underline{\text { Signifikanz }}$ \\
\hline Parietallappen & $6,201(3)$ & .102 & $3,78(1)$ & .052 & 0,459 (3) & .928 \\
\hline Superiorer PL & $6,528(3)$ & .089 & $1,839(1)$ & .175 & $0,190(3)$ & .979 \\
\hline Inferiorer PL & $1,875(3)$ & .599 & $0,330(1)$ & .566 & $1,242(3)$ & .742 \\
\hline
\end{tabular}

" Testverfahren und Prüfgröße = zweifaktorielle, nonparametrische Kovarianzanalyse, $t$-Wert; $d \mathbf{f}=$ Freiheitsgrade. 
Anzumerken ist allerdings, dass sich ein signifikanter Subgruppen-Effekt im Hinblick auf das globale Cortexvolumen zeigte $(\mathrm{t}=4,329 ; \mathrm{p}=.001)$, welche in der vorliegenden Analyse als Kovariate fungierte.

\subsubsection{Klassische neuropsychologische Verfahren}

Die neuropsychologischen Testleistungen von 38 schizophrenen und 38 gesunden Testpersonen in der WMS-R sowie 39 schizophrenen und 38 gesunden Testpersonen im TMT A und B wurden mit Hilfe eines non-parametrischen Kruskal-Wallis-H-Tests verglichen ${ }^{24}$. Tabelle 25 gibt einen Überblick:

Tabelle 25: $\quad$ Neuropsychologische Testleistungen schizophrener \& gesunder Probanden

\begin{tabular}{|c|c|c|c|c|c|c|}
\hline$\underline{\text { Gruppe: }}$ & \multicolumn{2}{|c|}{$\begin{array}{c}\text { Schizophrene } \\
\text { Patientengruppe }\end{array}$} & \multicolumn{2}{|c|}{$\begin{array}{l}\text { Gesunde } \\
\text { Kontrollgruppe }\end{array}$} & \multicolumn{2}{|c|}{$\frac{\text { Testverfahren und }}{\text { Prüfgröße }^{\#}}$} \\
\hline & MW & $\underline{\mathrm{S}}$ & MW & $\underline{\mathrm{S}}$ & $\chi^{2}$-Wert & Signifikanz \\
\hline $\begin{array}{c}\text { WMS-R } \\
\text { Digit Span forward }\end{array}$ & 7,79 & 1,58 & 7,97 & 1,64 & 0,211 & $p=.646$ \\
\hline $\begin{array}{c}\text { WMS-R } \$ \\
\text { Digit Span backward }\end{array}$ & 5,95 & 1,75 & 7,40 & 2,07 & 8,624 & $\mathbf{p}=.003 * *$ \\
\hline $\begin{array}{c}\text { WMS-R } \\
\text { Visual Memory Span } \\
\text { forward }\end{array}$ & 8,24 & 1,67 & 8,82 & 1,94 & 1,208 & $p=.272$ \\
\hline $\begin{array}{c}\text { WMS-R } \\
\text { Visual Memory Span } \\
\text { backward }\end{array}$ & 7,39 & 1,72 & 8,26 & 1,94 & 5,894 & $p=.015^{*}$ \\
\hline $\begin{array}{c}\text { WMS-R } \\
\text { Logical Memory I }\end{array}$ & 24,16 & 8,33 & 31,92 & 5,42 & 17,653 & $\mathbf{p}<.001 * *$ \\
\hline $\begin{array}{c}\text { WMS-R }^{\$} \\
\text { Logical Memory II }\end{array}$ & 19,22 & 9,14 & 28,53 & 6,44 & 18,912 & $\mathbf{p}<.001 * *$ \\
\hline $\begin{array}{c}\text { WMS-R } \\
\text { Visual Reproduction I }\end{array}$ & 36,26 & 5,39 & 38,26 & 2,74 & 2,442 & $p=.118$ \\
\hline $\begin{array}{c}\text { WMS-R } \$ \\
\text { Visual Reproduction II }\end{array}$ & 31,29 & 9,21 & 36,97 & 4,71 & 7,769 & $\mathbf{p}=.005^{*} *$ \\
\hline TMT-A $^{\S}$ & $29,92 \mathrm{sec}$ & $12,23 \mathrm{sec}$ & $23,53 \mathrm{sec}$ & $6,86 \mathrm{sec}$ & 6,448 & $p=.011 *$ \\
\hline TMT-B $^{\S}$ & $65,59 \mathrm{sec}$ & $38,11 \mathrm{sec}$ & $41,45 \mathrm{sec}$ & $16,25 \mathrm{sec}$ & 11,689 & $\mathbf{p}=.001 * *$ \\
\hline
\end{tabular}

MW = Mittelwert; $\mathbf{s}=$ Standardabweichung; ${ }^{\#}$ Testverfahren und Prüfgröße = Kruskal-Wallis-H-Test, $\chi^{2} ;{ }^{\$}$ WMS-R = Wechsler-Memory-Scale-Revised; Kennwert: Rohpunkte; ${ }^{{ }^{\S}}$ TMT = Trail Making-Test; Kennwert: Sekundenzahl; * = signifikant bei $\alpha<0.05$; $* *$ signifikant bei $\alpha<0.01$.

\footnotetext{
${ }^{24}$ Anzumerken ist, dass für die schizophrene Probandengruppe aufgrund eines drop-outs nur 37 Datensätze in den WMS-R-Subtest Logical Memory II eingingen.
} 
Wie aus Tabelle 25 ersichtlich, waren die schizophrenen Probanden durch deutliche Defizite in einer Vielzahl mnestischer Funktionen charakterisiert: Im Vergleich zu Kontrollpersonen wiesen sie schwächere Leistungen in verbalen (digit span backward: $\chi^{2}=$ 8,624, $\mathrm{p}=.003$; logical memory $\mathrm{I}: \chi^{2}=17,653, \mathrm{p}<.001$ ) sowie in visuellen (visual memory span backward: $\chi^{2}=5,894, \mathrm{p}=.015$ ) (Kurzzeit-)Gedächtnisfunktionen auf. Zudem zeigten sich markante Leistungseinbußen in verbalen (logical memory II: $\chi^{2}=$ 18,$912 ; \mathrm{p}<.001$ ) und visuellen (visual reproduction II: $\chi^{2}=7,769, \mathrm{p}=.005$ ) Langzeitgedächtnisprozessen. Darüber hinaus ließen sich deutliche Defizite in der visuomotorischen Aktionsfähigkeit beobachten (TMT A: $\chi^{2}=6,448, p=.011$; TMT B: $\chi^{2}$ $=11,689 ; \mathrm{p}=.001)$. 


\section{Diskussion}

\subsection{Zentrale Forschungshypothesen}

\subsubsection{Visuell-räumliche Navigationsleistung (Hypothesen 1 und 2)}

In der vorliegenden Arbeit konnten deutliche Defizite in der Navigationsleistung schizophrener Patienten beobachtet werden. Schizophrene Patienten benötigten mehr Zeit, um den Zielpunkt innerhalb der virtuellen Testumgebungen $\mathrm{zu}$ finden, konnten bei mehrmaligem Durchlaufen der Wegenetze weniger Lerngewinne verbuchen und machten beim Wiederfinden des Ziels mehr Fehler als gesunde Kontrollpersonen.

Die Testergebnisse decken sich folglich mit einer Vielzahl an Arbeiten, die Defizite in der visuell-räumlichen Informationsverarbeitungsleistung schizophrener Probanden nachweisen konnten (z.B. Park \& Holzman, 1992; Park et al., 1995; Carter et al., 1996; Fleming et al., 1997; Tan et al., 2005). Im Gegensatz zu diesen konventionellen Arbeiten besitzt die vorliegende Studie den Vorteil, dass sie mit dem VR-Paradigma als eine der ersten ein methodisches Design mit maximaler Authenzität und hoher ökologischer sowie prognostischer Validität zur Überprüfung visuell-räumlicher Defizite bei der Schizophrenie einsetzt (vgl. Ruddle et al., 1997; van Veen et al., 1998; Waller et al., 1998; Costa et al., 2002; Mehlitz, 2004). Im Einklang mit den Befunden von Ku et al. (2003), Astur et al. (2004) und Kurtz et al. (2004) ließen sich innerhalb dreidimensionaler virtueller Testumgebungen deutliche Navigationsdefizite bei schizophrenen Patienten nachweisen, was darauf schließen lässt, dass sie auch im täglichen Leben durch Orientierungs- und Navigationsprobleme charakterisiert sind, die ihre Alltagsfähigkeit stark einschränken (vgl. Skelton et al., 2000; Spiers et al., 2001).

\section{Ego- und allozentrische Navigationsprozesse}

Neben dem Einsatz der VR-Technologie besitzt die vorliegende Arbeit den Vorzug, dass sie erstmals ego- und allozentrische Navigationsleistungen bei der Schizophrenie untersuchte. Mit dem Haupteffekt „Gruppe“ konnte gezeigt werden, dass schizophrene Testpersonen in beiden virtuellen Testumgebungen schlechtere Leistungen aufwiesen als gesunde Kontrollpersonen. Unabhängig davon, ob Wegmarken zur Verfügung standen (Bedingung „VR-Park“) oder nicht (Bedingung „VR-Labyrinth“), benötigten schizophrene Probanden mehr Zeit, um den Zielpunkt zu finden, machten beim Durchlaufen der Wegenetze mehr Fehler und konnten nur geringere Lerngewinne verbuchen als gesunde Probanden. 
Wie bereits erläutert, weisen schizophrene Probanden Defizite in visuell-räumlichen Informationsverarbeitungsprozessen auf (vgl. 1.1.3. und 1.1.4.3.3.). Im Einklang damit ließen sich in der vorliegenden Arbeit Defizite in allozentrischen Navigationsleistungen beobachten, die primär auf visuell-räumlichen Hinweisreizen - den Wegmarken - basieren (vgl. Kitchin, 1994; Aguirre et al., 1998; Gillner \& Mallot, 1998; Save \& Poucet, 2000; Hartley et al., 2003). Dass sich darüber hinaus auch Leistungseinbußen in der egozentrischen Navigationsleistung schizophrener Probanden zeigten, deckt sich mit den unter 1.1.4.3.2. und 1.1.4.3.3. resümierten Befunden, dass auch propriozeptive Informationsverarbeitungsprozesse bei der Schizophrenie betroffen sind. Prozesse, die die Grundlage egozentrischer Kognitionsleistungen bilden (vgl. Burgess et al., 2001a,b; Hartley et al., 2003; Weniger \& Irle, 2006).

Die vorliegende Arbeit unterstreicht somit die Befunde zu defizitären visuell-räumlichen und propriozeptiven Kognitionsleistungen schizophrener Patienten, die sich im vorliegenden Setting als eingeschränkte Navigationsleistungen im ego- und allozentrischen Raum widerspiegeln.

\section{Variabilität der Versuchsergebnisse}

Bei der Interpretation der Versuchsergebnisse ist allerdings auf eine relative große Varianz des visuell-räumlichen Leistungsprofils zu achten. Sowohl in der Patienten-, als auch in der Kontrollgruppe variierte die Navigationsleistung zwischen verschiedenen Testpersonen stark. Wie später noch genauer diskutiert wird, handelt es sich bei der Schizophrenie um ein stark heterogenes Krankheitskonzept, innerhalb dessen der Ausprägungsgrad neuropsychologischer Defizite deutlich divergieren kann (vgl. Andreasen et al., 1990; McCarley et al., 1999; O’Carroll, 2000). Im Einklang damit bewegte sich die Navigationsfähigkeit einiger Patienten in der vorliegenden Arbeit durchaus im Normbereich, während andere Patienten deutlich eingeschränkt waren. Festzuhalten bleibt, dass schizophrene Probanden in ihrer Gesamtheit Navigationsdefizite aufweisen, dass diese Defizite innerhalb der „Gruppe der Schizophrenien“ (Bleuler, 1911) jedoch stark variieren können.

\section{Effekte der Testumwelt}

Ergänzend zu dem Haupteffekt „Gruppe“ ließ sich mit dem Faktor „Testumwelt“ ein zweiter Haupteffekt beobachten. Anhand der Indikatoren „Fehlersumme I“ und „Zeit I“ zeigte sich, dass alle Testpersonen bessere Navigationsleistungen im virtuellen Park als im virtuellen Labyrinth aufwiesen. 
Dieses Ergebnis deckt sich mit dem Hinweis verschiedener Autoren, dass Navigationsprozesse im egozentrischen Raum anspruchsvoller sind als Navigationsprozesse im allozentrischen Raum (vgl. Distler et al., 1998; Gillner \& Mallot, 1998; Weniger \& Irle, 2006): „...empirical evidence suggests, that users frequently have problems navigating VEs, when supplementary aids (maps, landmarks...) are not provided... “ (Ruddle et al., 1997, S. 143). Im Einklang damit stuften sowohl schizophrene, als auch gesunde Testpersonen die Aufgabenschwierigkeit im Labyrinth höher ein als im virtuellen Park (vgl. Tabelle 13).

Durch die höhere Aufgabenschwierigkeit begingen sowohl schizophrene, als auch gesunde Testpersonen im virtuellen Labyrinth mehr Fehler bei der Suche nach dem Zielpunkt und benötigen mehr Zeit, um diesen zu erreichen.

\subsubsection{Parietale Cortexvolumina (Hypothese 3)}

In der vorliegenden Arbeit konnten bei den schizophrenen Patienten mit dem Haupteffekt „Gruppe“ signifikante Volumenreduktionen im globalen Parietallappen sowie in seinem superioren Subareal beobachtet werden.

Diese Befunde decken sich zum einen mit den Reviews von Pearlson \& Marsh (1999) und Shenton et al. (2001) sowie mit den Arbeiten von Cannon et al. (2001, 2002), die ebenfalls Volumenreduktionen im globalen und superioren Parietallappen finden konnten und liefern zum anderen direkte empirische Evidenz für eine pathologische Beteiligung parietaler Cortexareale an der schizophrenen Psychopathologie (vgl. Goldstein et al., 1999; Frederikse et al., 2000; Shenton et al., 2001; Danckert et al., 2004). Neben frontalen und temporalen Arealen (vgl. 1.1.4.) scheinen auch Subareale des parietalen Cortex in ihrem Volumen reduziert zu sein.

Wie bereits unter 1.1.4.3.3. erläutert, ist der parietale Cortex an der Visuo- und Psychomotorik, propriozeptiven, egozentrischen und visuell-räumlichen, allozentrischen Kognitionsleistungen sowie an deren Dysfunktion bei der Schizophrenie maßgeblich beteiligt.

In der vorliegenden Arbeit konnten neben Volumenreduktionen des globalen Parietalcortex insbesondere Reduktionen des superior-parietalen Subareals beobachtet werden. Typische Funktionen des superioren Parietallappens sind visuell-räumliche Aufmerksamkeits- und Informationsverarbeitungsleistungen (Posner \& Petersen, 1990; Paulus et al., 2002), visuomotorische Prozesse (Driver \& Mattingley, 1998) sowie propriozpetive und 
egozentrische Kognitionsleistungen (Culham \& Kanwisher, 2001; Wolpert et al., 1998). Die gefundenen Volumenreduktionen können folglich als die neuronale Grundlage typischer (superior-)parietaler Dysfunktionen bei der Schizophrenie aufgefasst werden (vgl. Cannon et al., 2002).

Insgesamt sprechen die vorliegenden Daten für eine parietale Volumenreduktion und somit für die Beteiligung des Parietalcortex an der schizophrenen Psychopathologie.

\subsubsection{Zusammenhänge Neuropsychologie - Hirnvolumetrie (Hypothesen 4 und 5)} Egozentrische Navigationsleistung

In der schizophrenen Probandengruppe ließen sich verschiedene Korrelationen zwischen der egozentrischen Navigationsleistung und parietalen Cortexvolumina feststellen, von denen alle hypothesenkonform $\mathrm{zu}$ interpretieren sind: Je kleiner das parietale Volumen war, desto schlechter war auch die Navigationsleistung im egozentrischen Raum.

Die (defizitäre) egozentrische Navigationsleistung schizophrener Patienten wies die deutlichsten Zusammenhänge zum (reduzierten) Volumen des globalen Parietallappens beider Hemisphären, zum rechten, superioren Parietallappen sowie zum linken, inferioren Parietallappen auf. Dies deckt sich mit der unter 1.1.4.3.3. und 5.1.2. dargestellten funktionellen Spezialisierung parietaler (Sub-)Areale auf egozentrische, propriozeptive Kognitionsleistungen (vgl. auch Clower et al., 1996; Berlucchi \& Aglioti, 1997; Wolpert et al., 1998; Culham \& Kanwisher, 2001; Danckert et al., 2004). Es scheint also als seien Volumenanomalien des Parietalcortex für die egozentrischen Navigationsdefizite verantwortlich.

\section{Allozentrische Navigationsleistung}

Die defizitäre allozentrische Navigationsleistung schizophrener Probanden korrelierte ebenfalls mit Volumenreduktionen in verschiedenen parietalen Cortexarealen, wobei sich erneut aussschließlich hypothesenkonforme Zusammenhänge zeigten: Je kleiner das parietale Hirnvolumen war, desto kleiner war auch der Lerngewinn beziehungsweise desto höher die Fehlerzahl und die benötigte Zeit bis zum Finden des Zielpunktes im „virtuellen Park“.

Im Einklang mit den Befunden zur egozentrischen Navigation korrelierten vor allem die Volumina des globalen Parietallappens beider Hemisphären sowie der rechte, superiore Parietallappen mit der allozentrischen Navigationsleistung der Patienten. Auch die Defizite in der allozentrischen Navigation scheinen also mit (superior-)parietalen 
Volumenreduktionen zusammenzuhängen, was sich mit der Beobachtung deckt, dass visuell-räumliche Kognitionsprozesse, die der allozentrischen Navigation zugrunde liegen, primär durch Parietallappenareale realisiert werden (vgl. Büchel et al., 1998; Mesulam, 1998; Huguelet et al., 2000; Paulus et al., 2002).

Zusammenfassend weisen die Navigationsdefizite schizophrener Probanden im ego- und allozentrischen Raum also eine funktionale Beziehung zu Volumenreduktionen im globalen sowie im superioren und inferioren Parietallappen auf. Defizite in ego- und allozentrischen Informationsverarbeitungsprozessen scheinen ihre neuronale Entsprechung in parietalen Volumenreduktionen zu besitzen.

\section{Lateralisierungseffekte}

Auffällig ist, dass vor allem rechtshemisphärische Parietallappenareale mit der allozentrischen Navigationsleistung schizophrener Probanden korrelieren, während die egozentrische Navigationsleistung mit rechts- und linkshemisphärischen Parietallappenarealen assoziiert ist.

Eine primär rechtsseitige Lateralisierung allozentrischer Navigationsprozesse kann damit begründet werden, dass Probanden im virtuellen Park vor allem visuell-räumliche Orientierungsstrategien nutzten, um den Zielpunkt zu finden. Strategien, die primär rechtslateralisiert sind (vgl. Mesulam, 1985; Petersen et al., 1989; Posner \& Petersen, 1990; Kolb \& Whishaw, 1996; Wood et al., 2002).

Wie bereits unter 5.1.1. diskutiert, zeichnet sich die egozentrische Navigationsaufgabe durch eine höhere Aufgabenschwierigkeit aus, so dass im Gegensatz zur allozentrischen Navigation wahrscheinlich nicht nur nonverbale, sondern auch ergänzende verbale Navigationsstrategien (zum Beispiel eine Aufeinanderfolge von „rechts-links“Kombinationen) zum Einsatz kamen, die primär linkslateralisiert sind (vgl. Mesulam, 1985; Kolb \& Whishaw, 1996; Wood et al., 2002). Ein vermehrter Einsatz verbaler Navigationsstrategien im Labyrinth deckt sich auch mit den Ergebnissen des Nachbefragungsbogens (vgl. Tabelle 13). Je nachdem, welche Navigationsstrategien die Testpersonen also benutzten, wurden folglich (primär) rechts- beziehungsweise linksparietale Areale in Anspruch genommen, denn: „...there is general agreement regarding the primacy of the left hemisphere in verbal cognitive and language functions and of the right hemisphere for spatial functions" (Saykin et al., 1991, S. 618). 


\section{Das parieto-hippocampale Navigationsnetzwerk}

Wie die bisherige Diskussion zeigt, korrelieren die Volumina parietaler Cortexareale sowohl mit den ego-, als auch mit den allozentrischen Navigationsleistungen schizophrener Probanden. Eine exklusive „Spezialisierung“ parietaler Areale auf egozentrische Navigationsprozesse, wie sie ursprünglich postuliert wurde (vgl. Tabelle 3), konnte in der vorliegenden Arbeit folglich nicht bestätigt werden. Vielmehr sprechen die vorliegenden Daten für eine parietale Beteiligung an den verschiedenen Formen der Navigation (vgl. Duhamel et al., 1992a; Andersen, 1997; Snyder et al., 1998; Save \& Poucet, 2000) und gegen eine klare funktionelle und neuronanatomische Trennung des parieto-hippocampalen Navigationsnetzwerks (vgl. Aguirre et al., 1998; Save \& Poucet, 2000; Burgess et al., 2001a; Shelton \& Gabrieli, 2002).

\subsubsection{Zusammenfassung zentrale Forschungshypothesen}

Aus der bisherigen Diskussion wird deutlich, dass die zentralen Versuchshypothesen bestätigt werden können: Schizophrene Probanden weisen defizitäre Navigationsleistungen im ego- und allozentrischen Raum (Hypothesen 1 und 2) sowie Volumenreduktionen im globalen und superioren Parietalcortex auf (Hypothese 3). Zudem besteht zwischen den neuropsychologischen und den hirnanatomischen Variablen ein funktioneller Zusammenhang: Je kleiner das parietale Cortexvolumen ist, desto schlechter ist die visuellräumliche Navigationsleistung im ego- und allozentrischen Raum (Hypothesen 4 und 5).

Die aktuellen Befunde untermauern somit die These, dass parietale Cortexareale an der schizophrenen Psychopathologie und insbesondere an Defiziten in allo- und egozentrischen Naviationsleistungen maßgeblich beteiligt sind.

\subsection{Explorative und validierende Hypothesen}

\subsubsection{Hippocampale Areale (Hypothesen 6 und 7)}

Entsprechend einer Vielzahl an Studien (vgl. Bogerts et al., 1993; Nelson et al., 1998; Velakoulis et al., 2001; Szeszko et al., 2002, 2003) konnten auch in der vorliegenden Arbeit deutliche, bilaterale Volumenreduktionen des Hippocampus bei der schizophrenen Stichprobe festgestellt werden. Entgegen der Versuchshypothesen und der Annahme eines parieto-hippocampalen Navigationsnetzwerks ließen sich allerdings weder in der Kontrollgruppe, noch in der Patientengruppe deutliche Zusammenhänge zur ego- oder allozentrischen Navigationsleistung ausmachen. 
Wie unter 1.2.1.2.3. erläutert, besteht eine allgemeine Uneinigkeit darüber, welche medialtemporalen (und parietalen) Areale für die visuell-räumliche Navigationsleistung von Relevanz sind. Im Einklang mit den aktuellen Befunden sprechen einige neuere Arbeiten gegen eine maßgebliche Beteiligung hippocampaler Areale beziehungsweise für die (primäre) Relevanz parahippocampaler Areale (vgl. Aguirre et al., 1996; Aguirre et al., 1998; Maguirre et al., 1998b; Epstein \& Kanwisher, 1998; Grön et al., 2000; Mellet et al., 2000; Burgess et al., 2001b; Weniger \& Irle, 2006). Die Klärung, ob ego- und allozentrische Navigationsleistungen durch ein parieto-hippocampales oder aber eher durch ein parieto-parahippocampales Netzwerk realisiert wird, sollte Ziel weiterführender Studien sein - zumindest in der vorliegenden Arbeit ließen sich keine funktionalen Beziehungen zum Hippocampus nachweisen.

\subsubsection{Schizophrene Subgruppen (Hypothesen 8 und 9)}

Im Hinblick auf die visuell-räumliche Navigationsleistung bestätigte die explorative Subtypenanalyse im Wesentlichen die bereits unter 5.1.1. resümierten Befunde der Hauptanalyse: Mit dem Haupteffekt „Subgruppe“ zeigte sich, dass gesunde Testpersonen bessere ego- und allozentrische Navigationsleistungen als paranoide oder desorganisierte Patienten aufwiesen, mit dem Haupteffekt „Testumwelt“ ließen sich im Vergleich zum virtuellen Labyrinth erneut überlegene Navigationsleistungen im virtuellen Park nachweisen.

Im Hinblick auf die Frage nach differentiellen Subtypenprofilen scheint vor allem ein Interaktionseffekt wesentlich, der darauf hinwies, dass paranoid-schizophrene Testpersonen vor allem Navigationsdefizite im egozentrischen Raum aufwiesen, während desorganisiert-schizophrene Patienten insbesondere durch Defizite im allozentrischen Raum charakterisiert waren.

Innerhalb der hirnanatomischen Subtypenanalyse konnten keine differentiellen Effekte gefunden werden; die parietalen Cortexvolumina unterschieden sich zwischen den Subgruppen nicht signifikant. Anzumerken ist allerdings, dass das globale Cortexvolumen, das innerhalb der hirnanatomischen Berechnungen als Kovariate fungierte, einen signifikanten Effekt ausübte und sich zwischen den Subgruppen deutlich unterschied. Auch wenn der Focus der vorliegenden Arbeit auf parietalen Hirnstrukturen lag, weist dies doch darauf hin, dass die schizophrenen Subtypen durch unterschiedliche cortikale Anomalien charakterisiert sind. 
Sowohl die neuropsychologische, als auch die hirnanatomische Subtypenanalyse gibt im Einklang mit den Befunden anderer Autoren (vgl. Höschel et al., 1998; Claussen, 2004; Weniger et al., 2004a) folglich zumindest Hinweise darauf, dass die verschiedenen schizophrenen Subtypen durch differentielle neuropsychologische und neuroanatomische Profile charakterisiert sind - auch wenn die Effekte eher schwach sind und die schizophrenen Subgruppen durch relativ kleine Zellenbesetzungen repräsentiert wurden. Weiterführende Studien mit einer größeren Anzahl an Testpersonen scheinen in diesem Zusammenhang lohnenswert, um die Frage nach subtypenspezifischen neuropsychologischen und neuroanatomischen Profilen differenzierter untersuchen und auch auf inferenzstatistischer Ebene noch besser absichern zu können.

\subsubsection{Klassische neuropsychologische Verfahren (Hypothesen 10 und 11)}

Entsprechend den Versuchshypothesen 10 und 11 ließen sich deutliche Einschränkungen in verbalen und visuellen Langzeit- und Kurzzeitgedächtnisprozessen sowie in der visuomotorischen Aktionsgeschwindigkeit schizophrener Testpersonen beobachten. Die beobachteten Defizite decken sich mit einer Vielzahl an neuropsychologischen Schizophreniestudien (vgl. Saykin et al., 1991, 1994; Heinrichs \& Zakzanis, 1998; Rund, 1998; Sanfilipo et al., 2002; Zihl, 2004d) und sprechen somit für die Repräsentativität der aktuellen Stichprobe sowie für die Validität der vorliegenden Versuchsergebnisse.

\subsection{Zusammenfassung}

Insgesamt können die Versuchshypothesen der vorliegenden Arbeit größtenteils bestätigt werden: Schizophrene Probanden wiesen defizitäre ego- und allozentrische Navigationsleistungen (Hypothesen 1 und 2) sowie parietale Volumenreduktionen (Hypothese 3) auf; die korrelative Analyse wies des Weiteren darauf hin, dass ein funktionaler Zusammenhang zwischen den neuropsychologischen und den hirnantomischen Befunden besteht: Vor allem Patienten mit geringen parietalen Cortexvolumina waren durch defizitäre ego- und allozentrische Navigationsleistungen charakterisiert (Hypothesen 4 und 5). Im Gegensatz dazu konnten keine Zusammnhänge zu (deutlich volumenreduzierten) hippocampalen Arealen gefunden werden (Hypothesen 6 und 7).

Innerhalb der explorativen Subtypenanalyse ergaben sich Hinweise auf differentielle egound allozentrische Navigationsdefizite in der paranoiden und der desorganisierten Patientengruppe. Differentielle Parietallappenvolumina ließen sich innerhalb der 
Subgruppen nicht beobachten, allerdings deuteten sich Effekte im globalen Cortexvolumen an (Hypothesen 8 und 9).

Schließlich konnten bei schizophrenen Testpersonen Defizite in mnestischen und visuomotorischen Kognitionsleistungen nachgewiesen werden. Defizite, die in der neuropsychologischen Schizophrenieliteratur relativ konsistent dokumentiert wurden und somit die Repräsentativität und Validität der aktuellen Versuchsergebnisse untermauern (Hypothesen 10 und 11).

\subsection{Abschließende Bemerkungen}

Wie bereits diskutiert, ließen sich in der vorliegenden Arbeit größtenteils hypothesenkonforme Ergebnisse beobachten. Kritisch sollte allerdings angemerkt werden, dass es sich zum Teil um sehr sensible Effekte handelt: So ließ sich die defizitäre Navigationsleistung schizophrener Probanden nicht mit allen Indikatoren finden, parietale Volumenreduktionen nicht in allen untersuchten Subarealen und korrelative Zusammenhänge nicht zwischen allen Indikatoren und allen Subarealen.

Dass sich keine durchgängigen Effekte zeigten, deckt sich mit weiten Bereichen der Schizophrenieliteratur: Im Hinblick auf spezifische neuropsychologische Variablen, auf neuroanatomische Größen und insbesondere hinsichtlich neuropsychologischneuroanatomisch-psychopathologischer Zusammenhänge finden sich immer wieder divergierende, inkonsistente Befunde (vgl. Buchanan et al., 1993; Harvey et al., 1993; Pearlson et al., 1996, 1997; Harrison, 1999; Pearlson \& Marsh, 1999; Shenton et al., 2001; Schultz et al., 2002). Selbst so robuste Befunde wie Defizite im „Trail Making Test A und B“ und in Aufmerksamkeitsfunktionen sowie Volumenreduktionen des Hippocampus, des DLPFC oder des STG konnten nicht konsistent gefunden werden (vgl. Chua \& McKenna, 1995; Maruff et al., 1995; Marsh et al., 1997; Pearlson et al., 1997; Buchanan et al., 1998; Buchanan et al., 2004; van Beilen et al., 2005). Ho et al. (2003, S. 591-592) resümieren „...the literature...seems to be conflicting and...each study seems to have its own unique combination of results...".

Die Ursache der inkonsistenten Befundlage liegt vor allem in eher kleinen, subtilen Effekten: Neuropsychologische Testleistungen und hirnanatomische Maße schizophrener Probanden weisen eine große Variationsbreite auf und können sich durchaus im Normbereich bewegen (Goldstein, 1986; Goldberg \& Seidman, 1991; Chua \& McKenna, 1995; Goldstein et al., 1999; Shenton et al., 2001): „,...one of the most important points is that there is substantial variability within the schizophrenic group..." (Goldberg \& 
Seidman, 1991, S. 580), „...many individuals with schizophrenia score within the normal range and some are well above average..." (Harrison, 1999, S. 601). Darüber hinaus finden sich auch in gesunden Probandengruppen große interindividuelle Variationen, so dass sich die pathologische und die Normkurve in weiten Teilen überlappen (vgl. Weinberger et al., 1992; Cannon et al., 2002). Folglich können schizophrene und gesunde Patientengruppen nur anhand relativ schwacher Effekte differenziert werden.

Das Auffinden dieser subtilen Effekte wird zudem angesichts einer großen Zahl an Moderatorvariablen erschwert, die die Gruppenunterschiede zwischen schizophrenen und gesunden Testpersonen leicht überdecken können, wie der folgende Überblick zeigt.

\section{Schizophrene Subtypen}

Zunächst ist es möglich, dass sich bestimmte psychopathologische, neuropsychologische oder neurobiologische Auffälligkeiten nicht bei allen schizophrenen Probanden, sondern lediglich bei bestimmten Subgruppen finden (z.B. Tamminga et al., 1992; Maruff et al., 2005). Wie bereits erläutert, handelt es sich bei der Schizophrenie um ein heterogenes Krankheitskonzept, das stark unterschiedliche pathologische Zustandsbilder in sich vereint: „Schizophrenia is likely to encompass a set of disorders that differ with respect to their underlying etiologies and pathophysiological mechanisms...” (Cho et al., 2004, S. 299; vgl. auch Andreasen et al., 1990; McCarley et al., 1999; O’Carroll, 2000).

Es besteht folglich eine große Einigkeit über die Notwendigkeit, die „Gruppe der Schiozphrenien“ (Bleuler, 1911) valide zu subtypisieren (vgl. Goldstein, 1986; Goldstein \& Tsuang, 1988; Goldberg \& Seidman, 1991; Shenton et al., 2001; Kirkpatrick et al., 2003). Dem steht jedoch eine große Uneinigkeit über die Art der Subtypisierung gegenüber: Mit Unterscheidungen wie Typ I vs. Typ II (Crow, 1987a; Crow \& Johnstone 1987b; McCarley et al., 1999), paranoid vs. nonparanoid (Rund, 1983; Cleghorn et al., 1990; Wilke et al., 2001; Shapleske et al., 2002; Maruff et al., 2005), deficit vs. non-deficit (Tamminga et al., 1992), childhood-onset vs. early-onset vs. adult-onset (Thompson et al., 2001; Collinson et al., 2003; Gogtay et al., 2003), first-episode vs. chronic schizophrenia (Wood et al., 2002; Gur et al., 1998; Slewa-Younan et al., 2004), good vs. poor outcome (Mitelman et al., 2003), hypofrontalem vs. hypoparietalem vs. normalem Typus (Kishimoto, 1987) oder elektrodermalen OR-(non-)respondern (vgl. Watzl \& Rist, 1997; Hahlweg, 2000) wurden sowohl psychopathologische und neurophysiologische, als auch Verlaufsmerkmale als Alternative zur DSM-Typisierung (z.B. Seltzer et al., 1997; Marsh 
et al., 1999; Claussen, 2004; Weniger 2004 a,b) herangezogen (für einen Überblick s. Goldstein \& Tsuang, 1988; Watzl \& Rist, 1997).

Wie unter 1.1.2. bereits erläutert, weisen faktorenanalytische Studien auf das Vorliegen dreier Faktoren - der Skala „Positivsymptomatik“, der Skala „Negativsymptomatik“ und der Skala „Desorganisation“ - hin (Liddle, 1987; Höschel et al., 1998). Wenn auch von empirischer Seite unterstützt und in einer größeren Zahl von Studien verwendet (z.B. Peled, 1999; Claussen, 2004), hat sich jedoch auch diese 3-Faktoren-Terminologie bisher nicht durchsetzen können. Bis heute besteht kein arbeitsgruppenübergreifender Konsens bezüglich der Subtypisierung der Schizophrenie. Dies erscheint allerdings wesentlich, um robustere und konsistentere Ergebnisse erzielen zu können als dies in der bisherigen Schizophrenieforschung der Fall ist.

\section{Geschlecht}

Neben der Zugehörigkeit zu unterschiedlichen schizophrenen Subtypen ist auch das Geschlecht der Testpersonen ein wichtiger moderierender Faktor. Zum einen weisen demographische Daten auf einen Geschlechtsdimorphismus bei der Schizophrenie hin: Männliche Patienten erkranken früher, besitzen bereits prämorbid ein schlechteres Funktionsniveau und sind zudem durch einen schwereren Krankheitsverlauf mit frühreren und längerfristigen Hospitalisierungen sowie einer schlechteren Prognose charakterisiert als weibliche Patienten (Watzl \& Rist, 1997; Hahlweg, 2000; Helmchen et al., 2000). Zum anderen konnte in einer Vielzahl an Arbeiten die Verschiedenheit der psychopathologischen, neuropsychologischen und hirnanatomischen Anomalien bei Männern und Frauen gezeigt werden (z.B. Raz \& Raz, 1990; Maruff et al., 1995; Reite et al., 1997; Collinson et al., 2003; Slewa-Younan et al., 2004). So finden beispielsweise Frederikse et al. (2000) Volumenreduktionen sowie eine anormale Asymmetrie des IPL ausschließlich bei männlichen Patienten, Gur et al. (2000) Volumenreduktionen der Amygdala bei Männern, aber Volumenvergrößerungen bei Frauen, Cowell et al. (1996) unterschiedliche psychopathologisch-neuroanatomische Korrelationen für Männer und Frauen und Szeszko et al. (2002) nur bei männlichen Probanden Korrelationen zwischen dem Hippocampusvolumen und exekutiven Funktionen.

Eine Reihe von Arbeiten bemühte sich, den Störfaktor „Geschlecht“ durch die Untersuchung rein männlicher Stichproben zu kontrollieren (z.B. Marsh et al., 1997, 1999; Niznikiewicz et al., 2000; Sanfilipo et al., 2002; Schultz et al., 2002; Onitsuka et al., 2004). Auch weiterführende Studien sollten Männer und Frauen möglicherweise als separate 
Gruppen untersuchen, da die Befunde insgesamt darauf hinweisen, dass ,...sex is an important dimension and may contribute to the heterogeneity of brain structure, symptoms and the interaction of these two in schizophrenia..." (Cowell et al., 1996, S. 804).

\section{Medikation}

Ein weiteres Problem besteht in der Medikation der schizophrenen Probanden, die aus ethischen Gründen weder experimentell variiert, noch kontrolliert werden kann. Folglich erhalten die verschiedenen Patienten in Schizophreniestudien üblicherweise unterschiedlich hohe Dosen an unterschiedlichen (a-)typischen Neuroleptika, zudem zum Teil eine Medikation mit Anticholinergika (z.B. Park \& Holzman, 1992; Fleming et al., 1997; Marsh et al., 1999; Paulus et al., 2002; Badcock et al., 2005). Dies war auch in der vorliegenden Arbeit der Fall. Da es Hinweise auf die Beeinflussung neuropsychologischer und neuronanatomischer Variablen durch die antipsychotische und anticholinerge Medikation gibt (vgl. Goldberg \& Seidman, 1991; Blanchard \& Neale, 1994; Maruff et al., 1995; Andreasen et al., 1997; Gur et al., 1998; Rund, 1998; Shenton et al., 2001; Lieberman et al., 2005), wäre eine bessere Kontrolle dieser Moderatorvariablen ein wichtiges Ziel weiterführender Arbeiten, um die Konfundierung schizophreniespezifischer Auffälligkeiten mit medikamentösen Einflüssen aufzuheben (vgl. Watzl \& Rist, 1997). Möglichkeiten hierfür finden sich in der Untersuchung von neuroleptika-naiven Patienten (z.B. Saykin et al., 1994; Andreasen et al., 1997; Salgado-Pineda et al., 2003; Szeszko et al., 2003) oder durch einen medikamentösen ,wash-out“ (z.B. Blanchard \& Neale, 1994; Carter et al., 1996).

\section{Krankheitsphase, Chronizität und Schweregrad der Erkrankung}

Weitere wichtige Moderatorvariablen finden sich in der Krankheitsphase, in der sich die Patienten zum Untersuchungszeitpunkt befinden, in der Krankheitsdauer, also der Chronizität sowie im Schweregrad der Schizophrenie (z.B. Goldberg \& Weinberger, 1986; Goldstein, 1986; Seltzer et al., 1997).

Es gibt Hinweise, dass das Symptommuster der Schizophrenie nicht statisch ist, sondern je nach Krankheitsphase variiert, so dass sich vor allem in Akutphasen psychopathologische Symptome und neuropsychologische sowie neurophysiologische Anomalien beobachten lassen, die sich in Remissionsphasen zum Teil wieder zurückbilden (Sweeney et al., 1991; Saykin et al., 1994; Maruff et al., 1995; Goldberg \& Seidman, 1998; Rund, 1998; Exner et al., 2004; Weniger et al., 2004b). Des Weiteren weist eine Vielzahl an Studien darauf hin, 
dass sich das Symptombild mit ansteigender Chronizität verschlechtert, sich also progressive Kranhkeitsmuster erkennen lassen (Rund, 1983; Turetsky et al., 1995; Marsh et al., 1999; Thompson et al., 2001; Wood et al., 2002; Ho et al., 2003; Zihl, 2004d; Lieberman et al., 2005). Zudem scheint das Ausmaß der Defizite mit dem Schweregrad der Erkrankung zu korrelieren (vgl. Marsh et al., 1999; Wilke et al., 2001; Harvey et al., 2003). Je nach Schweregrad der Schizophrenie und je nachdem, ob sich Patienten zum Untersuchungszeitpunkt in einer Akut- oder Remissionsphase, zu Beginn der Erkrankung oder innerhalb langjähriger Chronifizierung befinden, ist demnach mit unterschiedlich (starken) Effekten zu rechnen ${ }^{25}$.

Bei den Patienten der vorliegenden Arbeit handelte es sich um nicht-chronifizierte Patienten, die sich zum Erhebungszeitpunkt in einer akuten psychotischen Episode nach initialer Stabilisierung befanden. Ob sich die gefundenen neuropsychologischen und hirnanatomischen Anomalien auch bei remittierten Patienten oder (eventuell noch stärker) bei solchen mit einer chronifizierten Schizophrenie zeigen lassen, kann anhand des vorliegenden Datenmaterials nicht entschieden werden. Weiterführende Längsschnittstudien und die Untersuchung chronisch erkrankter Patientengruppen wären wünschenswert, um die Generalisierbarkeit der Befunde und ihren fraglichen Traitcharakter untersuchen zu können (vgl. Cleghorn et al., 1990; Niznikiewicz et al., 2000; Velakoulis et al., 2001; Exner et al., 2004; Onitsuka et al., 2004).

\section{Auswahl der Kontrollgruppe}

Des Weiteren kann auch die Auswahl der Kontrollgruppe einen deutlichen Einfluss auf die Versuchsergebnisse nehmen (vgl. Bogerts et al., 1993; Chua \& McKenna, 1995). So wurden in der vorliegenden Studie beispielsweise Kontrollprobanden untersucht, die den schizophrenen Patienten in wichtigen soziodemographischen und neuropsychologischen Basisvariablen ähnelten. Die Kontrolle beziehungsweise die Angleichung von Variablen wie Geschlecht und Alter ist ein übliches Vorgehen beim Vergleich von Patienten und Kontrollpersonen (vgl. Lawrie \& Abukmeil, 1998). Diskutabel ist allerdings, ob ein matching von Intelligenz und Schulbildung sinnvoll ist, da eine eingeschränkte Intelligenz oder eine mangelnde Schulbildung möglicherweise einen Teil der schizophrenen Psychopathologie darstellen. Ihre Konstanthaltung könnte zur sogenannten „matching

\footnotetext{
25 Anzumerken ist, dass die Frage nach dem State- (vs. Trait-)Charakter sowie der Progredienz schizophreniespezifscher Defizite bisher nicht eindeutig geklärt werden konnte - es besteht eine lebhafte Diskussion: „...the stability of functioning is uncertain...the issue of state versus trait is clearly unresolved..." (Goldberg \& Seidman, 1991, S. 555; vgl. auch Rund, 1998; Claussen, 2004; O’Carroll, 2000; Zihl, 2004d).
} 
fallacy“ führen, bei der bestehende Effekte durch die Selektion einer unterdurchschnittlich begabten Kontrollgruppe (beziehungsweise einer überdurchschnittlich begabten Patientengruppe) überdeckt werden (vgl. Andreasen et al., 1990; Saykin et al., 1991; Schlaepfer et al., 1994; Heinrichs \& Zakzanis, 1998; Huguelet et al., 2000). „...group matching has been a thorny issue in schizophrenia research...since Paul Meehl called attention to the „matching fallacy“ - that groups may be overmatched on a variable that is not independent of the illness, per se...matching patients and normal controls on education or IQ may cause mismatching of theoretically expected cognitive ability..." (Snitz et al., 2005, S. 181).

Die Auswahl einer geeigneten Kontrollgruppe ist also ein wesentlicher Einflussfaktor jeder Schizophreniestudie. In der vorliegenden Arbeit wurden mit einer Angleichung von IQ und Schulbildung eine eher subnormale Kontrollgruppe beziehungsweise eine supranormale Patientengruppe untersucht. Dass sich trotz dieser eher konservativen Auswahl, die das Auftreten eines Beta-Fehlers begünstigt, deutliche Effekte zeigen, spricht für die Validität der vorliegenden Befunde.

Um deutlichere Effekte erzielen zu können, erscheint für zukünftige Studien alternativ ein matching anhand des geschätzten prämorbiden IQ's mit Hilfe des National Adult Reading Test (NART; Nelson, 1982) sinnvoll (vgl. Andreasen et al., 1990; Velakoulis et al., 2001).

\section{Methodische Moderatorvariablen}

Neben den genannten Stichprobenvariablen liegt die Ursache für die inkonsistente Befundlage schließlich auch in der methodischen Divergenz verschiedener Arbeiten: Neuropsychologische Funktionen werden häufig nicht einheitlich definiert und in verschiedenen Studien mit Hilfe heterogener Testsysteme, die nur bedingt miteinander vergleichbar sind, operationalisiert (vgl. Goldberg \& Weinberger, 1986; Levin \& Yurgelun-Todd, 1989; Maruff et al., 1995; Gitelman et al., 1999; Kinderman et al., 2004; Badcock et al., 2005). Heinrichs und Zakzanis (1998, S. 429) bemängeln anlässlich ihres Reviews: „organizing the myriad of neurocognitive test variables reported in the literature into a coherent classification was a major challenge“. Des Weiteren variieren auch die psychopathologischen Testsysteme verschiedener Arbeitsgruppen (z.B. Carter et al., 1996 vs. Huguelet et al., 2000). Zudem werden mit der Magnetresonanztomographie (MRT), der Computertomographie (CT), dem Diffusion Tensor Imaging (DTI), der funktionellen Magnetresonanztomographie (fMRT), der Positronen-Emissionstomographie (PET), der Elektroencephalographie und den Ereigniskorrelierten Potentialen (EEG und EKP), der 
Magnetencepalographie (MEG) sowie der Transkraniellen Magnetstimulation (TMS) heterogene neurobiologische Verfahren zur Erfassung struktureller und funktioneller Cortexcharakteristika eingesetzt (vgl. Tamminga et al., 1992; Reite et al., 1997; McCarley et al., 1999; Kubicki et al., 2002; MacDonald \& Paus, 2003; Pantelis et al., 2003; Onitsuka et al., 2004; Königsberg et al., 2005; Tendolkar et al., 2005). Dabei ist unklar, ob und in welcher Form ein Zusammenhang zwischen den strukturellen und funktionellen Anomalien besteht: „...it is unknown what realationship the structural and functional abnormalities bear to one another and which, if either, is primary... "(Pearlson \& Marsh, 1999, S. 631; vgl. auch Buchanan et al., 1998; Harrison, 1999; Maruff et al., 2005). Schließlich besteht selbst innerhalb desselben neurobiologischen Verfahrens eine große Variabilität, zum Beispiel bezüglich der Definition und Segmentierung des interessierenden Hirnareals (des ROIs), dem Vergleich von grauer Substanz, weißer Substanz und Cerebrospinalflüssigkeit oder in Parametern der Aufnahmetechnik wie der gewählten Schichtdicke und -anzahl oder der Stärke des Magnetfelds des Tomographiescans (vgl. Raz \& Raz, 1990; Buchanan et al., 1998; Nelson et al., 1998; Sullivan et al., 1998; Harrison, 1999; Pearlson, 2000; Shenton et al., 2001; Sigmundsson et al., 2001; Velakoulis et al., 2001).

Es wird deutlich, dass in der Methodenheterogenität der verschiedenen Arbeiten eine große Störvarianz begründet ist, die die Konsistenz von Versuchsergebnissen nachhaltig beeinträchtigen kann (vgl. Nelson et al., 1998; McCarely et al., 1999; Shenton et al., 2001). Eine bessere Standardisierung und Kombination der verschiedenen neuropsychologischen, psychopathologischen und neurobiologischen Methoden erscheint wesentlich, um die Befunde verschiedener Arbeitsgruppen zukünftig besser miteinander vergleichen und konsistentere Ergebnisse beobachten zu können.

\section{Zusammenfassung}

Wie aus der Diskussion ersichtlich, nehmen Größen wie die schizophrene Subgruppe, das Geschlecht, die Medikation, die Krankheitsphase, -chronizität und -intensität, die Auswahl der Kontrollgruppe sowie die verwandte Methodik also einen deutlichen Einfluss auf die erfasste Ausprägung der schizophrenen Psychopathologie, Neuropsychologie und Hirnanatomie (vgl. auch Goldberg \& Weinberger, 1986; Maruff et al., 1995; Heinrichs \& Zakzanis, 1998; McCarley et al., 1999). Sofern diese Variablen nicht kontrolliert werden, besteht das Risiko von Beta-Fehlern: Unterschiede zwischen der Patienten- und der 
Kontrollgruppe werden nicht gefunden, da die durch Moderatorvariablen bedingte Varianz größer ist als die psychopathologische Varianz.

Vor diesem Hintergrund und der Tatsache, dass die Schizophrenie auf neuropsychologischer und neuroanatomischer Ebene durch eher schwache Effekte charakterisiert ist, scheinen die vorliegenden Versuchsergebnisse - trotz ihrer zum Teil nur schwachen statistischen Signifikanz, insbesondere in der Subtypenanalyse - eine hohe inhaltliche Bedeutsamkeit zu besitzen.

Weiterführende Studien mit einer maximalen Kontrolle der beschriebenen Moderatorvariablen und einer hohen Stichprobengröße wären wünschenswert, um die beobachteten Befunde mit einer größeren Stabilität beobachten und Manfred Bleulers resignativem Resümee entgegentreten zu können, dass es sich bei den Befunden zur Schizophrenie um „winzige Körnchen Wissen in einem Meer von Unwissenheit“ handelt (nach Hahlweg, 2000, S. 313). 


\section{Literaturverzeichnis}

Abbott, C. \& Bustillo, J. (2006). What have we learned from proton magnetic resonance spectroscopy about schizophrenia? A critical update. Current Opinion in Psychiatry, 19, 135-139.

Aguirre, G.K., Detre, J.A., Alsop, D.C. \& D’Eposito, M. (1996). The parahippocampus subserves topographical learning in man. Cerebral Cortex, 6, 823-829.

Aguirre, G. \& D’Esposito, M. (1997). Environmental knowledge is subserved by separable dorsal/ventral neural areas. The Journal of Neuroscience, 17, 2512-2518.

Aguirre, G., Zarahn, E. \& D’Esposito, M. (1998). Neural components of topographical representation. Proceedings of the National Academy of Sciences of the United States of America, 95, 839-846.

Aguirre, G. \& D’Esposito, M. (1999). Topographical disorientation: A synthesis and taxonomy. Brain, 122, 1613-1628.

American Psychiatric Association (1994). Diagnostic and Statistical Manual of Mental Disorders (4th edition). Washington, D.C.: American Psychiatric Association.

Andersen, R.A. (1997). Multimodal integration for the representation of space in the posterior parietal cortex. Philosophical Transactions of the Royal Society of London, Series B: Biological Sciences, 352, 1421-1428.

Andreasen, N.C. (1983). Scale for the Assessment of Negative Symptoms (SANS). Iowa City: University of Iowa College of Medicine.

Andreasen, N.C. (1984). Scale for the Assessment of Positive Symptoms (SAPS). Iowa City: University of Iowa College of Medicine.

Andreasen, N.C., Ehrhardt, J.C., Swayze, V.W., Alliger, R.J., Yuh, W.T.C., Cohen, G. \& Ziebell, S. (1990). Magnetic resonance imaging of the brain in schizophrenia. Archives of General Psychiatry, 47, 35-44.

Andreasen, N.C., O’Leary, D., Flaum, M., Nopoulos, P., Watkins, G.L., Boles Ponto, L.L. \& Hichwa, R.D. (1997). Hypofrontality in schizophrenia: Distributed dysfunctional circuits in neuroleptic-naïve patients. The Lancet, 349, 1730-1734.

Association for Methodology and Documentation in Psychiatry (AMDP) \& Collegium Internationale Psychiatriae Scalarum (CIPS) (1990). Rating Scales for Psychiatry. Weinheim: Beltz.

Astur, R.S., Taylor, L.B., Mamelak, A.N., Philpott, L. \& Sutherland, R.J. (2002). Humans with hippocampus damage display severe spatial memory impairments in a virtual Morris water task. Behavioural Brain Research, 132, 77-84.

Astur, R.S., Germain, S., Mathalon, D.H., D’Souza, D.C., Krystal, J.K., Constable, R.T. \& Pearlson, G.D. (2004). Using virtual reality to investigate functioning of the hippocampus in schizophrenia. CyberPsychology \& Behavior, 7, 269. 
Badcock, J.C., Michie, P.T. \& Rock, D. (2005). Spatial working memory and planning ability: Contrasts between schizophrenia and bipolar I disorder. Cortex, 41, 753-763.

Bancaud, J., Brunet-Bourgin, F., Chauvel, P. \& Halgren, E. (1994). Anatomical origin of deja vu and vivid memories in temporal lobe epilepsy. Brain, 117, 71-90.

Barta, P.E., Pearlson, G.D., Powers, R.E., Richards, S.S. \& Tune, L.E. (1990). Auditory hallucinations and smaller superior temporal gyral volume in schizophrenia. The American Journal of Psychiatry, 147, 1457-1462.

Baumann, B. \& Bogerts, B. (1999). The pathomorphology of schizophrenia and mood disorders: Similarities and differences. Schizoprenia Research, 39, 141-148.

Baumann, S. (2004). Smoking cues in a virtual world provoke craving in cigarette smokers as demonstrated by neurobehavioral and fMRI data. CyberPsychology \& Behavior, 7, 270271.

Bäumler, G. (1974). Lern- und Gedächtnistest LGT-3. Handanweisung. Göttingen: Hogrefe.

Berlucchi, G. \& Aglioti, S. (1997). The body in the brain: Neural bases of corporeal awareness. Trends in Neurosciences, 20, 560-564.

Blakemore, S.-J. \& Frith, C.D. (2003). Self awareness and action. Current Opinion in Neurobiology, 13, 219-224.

Blanchard, J.J. \& Neale, J.M. (1994). The neuropsychological signature of schizophrenia: Generalized or differential deficit? The American Journal of Psychiatry, 151, 40-48.

Bleuler, E. (1911). Dementia praecox oder Gruppe der Schizophrenien. Leipzig: Franz Deuticke.

Bogerts, B., Lieberman, J.A., Ashtari, M., Bilder, R.M., Degreef, G., Lerner, G., Johns, C. \& Masiar, S. (1993). Hippocampus-amygdala volumes and psychopathology in chronic schizophrenia. Biological Psychiatry, 33, 236-246.

Bortz, J. (1993). Statistik für Sozialwissenschaftler (4.Auflage). Heidelberg: Springer Verlag.

Brähler, E., Holling, H., Leutner, D. \& Petermann, F. (Hrsg.) (2002a). Brickenkamp Handbuch psychologischer \& pädagogischer Tests 1 (3. Auflage). Hogrefe: Göttingen.

Brähler, E., Holling, H., Leutner, D. \& Petermann, F. (Hrsg.) (2002b). Brickenkamp Handbuch psychologischer \& pädagogischer Tests 2 (3. Auflage). Hogrefe: Göttingen.

Buchanan, R.W., Breier, A., Kirkpatrick, B., Elkashef, A., Munson, R.C., Gellad, F. \& Carpenter, W.T. (1993). Structural abnormalities in deficit and nondeficit schizophrenia. The American Journal of Psychiatry, 150, 59-65. 
Buchanan, R.W., Vladar, K., Barta, P.E. \& Pearlson, G.D. (1998). Structural evaluation of the prefrontal cortex in schizophrenia. The American Journal of Psychiatry, 155, 10491055 .

Buchanan, R.W., Francis, A., Arango, C., Miller, K., Lefkowitz, D.M., McMahon, R.P., Barta, P.E. \& Pearlson, G.D. (2004). Morphometric assessment of the heteromodal association cortex in schizophrenia. The American Journal of Psychiatry, 161, 322-331.

Büchel, C., Josephs, O., Rees, G., Turner, R., Frith, C.D. \& Friston, K.J. (1998). The functional anatomy of attention visual motion. Brain, 121, 1281-1294.

Buckwalter, J.G. \& Rizzo, A. (1997). Virtual reality and the neuropsychological assessment of persons with neurologically based cognitive impairments. In K.S. Morgan, H.M. Hoffman, D. Stredney \& S.J. Weghorst (Eds.), Studies in Health Technology and Informatics, Vol. 39 (pp. 17-33). Amsterdam: IOS Press.

Burgess, N., Maguire, E.A., Spiers, H.J. \& O’Keefe, J. (2001a). A temporoparietal and prefrontal network for retrieving the spatial context of lifelike events. NeuroImage, 14, 439-453.

Burgess, N., Becker, S., King, J.A. \& O’Keefe, J. (2001b). Memory for events and their spatial context: Models and experiments. Philosophical Transactions of the Royal Society of London B: Biological Sciences, 1493-1503.

Cannon, T.D., Thompson, P.M, van Erp, T., Toga, A.W, Poutanan, V.-P., Huttunen, M., Lonnqvist, J., Standerskjold-Nordenstam, C.-G., Narr, K.L., Khaledy, M., Zoumalan, C., Dail, R. \& Kaprio, J. (2001). A probabilistic atlas of cortical gray matter changes in monozygotic twins discordant for schizophrenia. NeuroImage, 6, S1034.

Cannon, T.D., Thompson, P.M., van Erp, T.G.M., Toga, A.W., Poutanan, V.-P., Huttunen, M., Lonnqvist, J., Standerskjold-Nordenstam, C.-G., Narr, K.L., Khaledy, M., Zoumalan, C.I., Dail, R. \& Kaprio, J. (2002). Cortex mapping reveals regionally specific patterns of genetic and disease-specific gray-matter deficits in twins discordant for schizophrenia. Proceedings of the National Academy of Sciences of the United States of America, 99, 3228-3233.

Carlin, A.S., Hoffman, H.G. \& Weghorst, S. (1997). Virtual reality and tactile augmentation in the treatment of spider phobia: A case report. Behaviour Research and Therapy, 35, 153-158.

Carrozzo, M. \& Lacquaniti, F. (1998). Virtual Reality: A tutorial. Electroencephalography and Clinical Neurophysiology, 109, 1-9.

Carter, C., Robertson, L., Nordahl, T., Chaderjian, M., Kraft, L. \& O’Shora-Celaya, L. (1996). Spatial working memory deficits and their relationship to negative symptoms in unmedicated schizophrenia patients. Biological Psychiatry, 40, 930-932.

Cho, R.Y., Gilbert, A. \& Lewis, D.A. (2004). The neurobiology of schizophrenia. In D.S. Charney \& E.J. Nestler (Eds.), Neurobiology of mental illness (chapter 22, pp. 299-310). New York: Oxford University Press. 
Chua, S.E. \& McKenna, P.J. (1995). Schizophrenia - a brain disease? The British Journal of Psychiatry, 166, 563-582.

Claussen, B. (2004). Kognitive Beeinträchtigungen bei schizophrenen Patienten: Eine Subtypenanalyse. Unveröffentlichte Diplomarbeit. Göttingen: Universität Göttingen, Fachbereich Neuropsychologie und Psychopathologie.

Cleghorn, J.M., Garnett, E.S., Nahmias, C., Firnau, G., Brown, G.M., Kaplan, R., Szechtman, H. \& Szechtman, B. (1989). Increased frontal and reduced parietal glucose metabolism in acute untreated schizophrenia. Psychiatry Research, 28, 119-133.

Cleghorn, J.M., Garnett, E.S., Nahmias, C., Brown, G.M., Kaplan, R.D., Szechtman, H., Szechtman, B., Franco, S., Dermer, S.W. \& Cook, P. (1990). Regional brain metabolism during auditory hallucinations in chronic schizophrenia. The British Journal of Psychiatry, $157,562-570$.

Clower, D.M., Hoffman, J.M., Votaw, J.R., Faber, T.L., Woods, R.P. \& Alexander, G.E. (1996). Role of posterior parietal cortex in the recalibration of visually guided reaching. Nature, 383, 618- 621.

Collinson, S.L., Mackay, C.E., James, A.C., Quested, D.J., Phillips, T., Roberts, N. \& Crow, T.J. (2003). Brain volume, asymmetry and intellectual impairment in relation to sex in early-onset schizophrenia. The British Journal of Psychiatry, 183, 114-120.

Corbetta, M. (1998a). Frontoparietal cortical networks for directing attention and the eye to visual locations: Identical, independent, or overlapping neural system. Proceedings of the National Academy of Sciences of the United States of America, 95, 831-838.

Corbetta, M., Akbudak, E., Conturo, T.E., Snyder, A.Z., Ollinger, J.M., Drury, H.A., Linenweber, M.R., Petersen, S.E., Raichle, M.E., Van Essen, D.C. \& Shulman, G.L. (1998b). A common network of functional areas for attention and eye movements. Neuron, 21, 761-73.

Cornblatt, B.A. \& Kelip, J.G. (1994). Impaired attention, genetics, and the pathopysiology of schizophrenia. Schizophrenia Bulletin, 20, 31-46.

Cornblatt, B.A. \& Malhotra, A.K. (2001). Impaired attention as an endophenotype for molecular genetic studies of schizophrenia. The American Journal of Medical Genetics, $105,11-15$.

Costa, R.M.E.M., de Carvalho, L.A.V., Drummond, R., Wauke, A.P.T. \& de sa Guimaraes, M. (2002). The UFRJ-UERJ group: Interdisciplinary virtual reality experiments in neuropsychiatry. CyberPsychology \& Behavior, 5, 423-431.

Cowell, P.E., Kostianovsky, D.J., Gur, R.C., Turetsky, B.I. \& Gur, R.E. (1996). Sex differences in neuroanatomical and clinical correlations in schizophrenia. The American Journal of Psychiatry, 153, 799-805. 
Crow, T.J. (1987a). Two syndromes of schizophrenia as one pole of the continuum of psychosis: A concept of the nature of the pathogen and its genomic locus. In F.A. Henn \& L.E. DeLisi (Eds.), Handbook of schizophrenia, Vol. 2: Neurochemistry and neuropharmacology of schizophrenia (chapter 2, pp. 17-48). Amsterdam: Elsevier Science Publishers.

Crow, T.J. \& Johnstone, E.C. (1987b). Schizophrenia: Nature of the disease and its biological correlates. In V.E. Mountcastle, F. Plum \& S.R. Geiger (Eds.), Handbook of physiology, section 1: The nervous system (chapter 21, pp. 843-869). Maryland: American Physiological Society.

Culham, J.C. \& Kanwisher, N.G. (2001). Neuroimaging of cognitive functions in human parietal cortex. Current Opinion in Neurobiology, 11, 157-163.

Danckert, J., Ferber, S., Doherty, T., Steinmetz, H., Nicolle, D. \& Goodale, M.A. (2002). Selective, non-lateralized impairment of motor imagery following right parietal damage. Neurocase, 8, 194-204.

Danckert, J., Saoud, M. \& Maruff, P. (2004). Attention, motor control and motor imagery in schizophrenia: Implications for the role of the parietal cortex. Schizophrenia Research, 70, 241-261.

Daprati, E., Franck, N., Georgieff, N., Proust, J., Pacherie, E., Dalery, J. \& Jeannerod, M. (1997). Looking for the agent: An investigation into conciousness of action and selfconsciousness in schizophrenic patients. Cognition, 65, 71-86.

Darken, R.P., Allard,T. \& Achille, L.B. (1998). Spatial orientation and wayfinding in large-scale virtual spaces: An introduction. Presence, 7, 101-7.

Davison, G.C. \& Neale, J.M. (2002). Schizophrenie. In M. Hautzinger (Hrsg.), Klinische Psychologie (6. Auflage, S. 356-400). Weinheim: Verlagsgruppe Beltz.

Distler, H.K., van Veen, H.A.H.C., Braun, J.S., Heinz, W., Franz, M.O. \& Bülthoff, H.H. (1998). Navigation in real and virtual environments: Judging orientation and distance in large-scale landscape. In M. Göbel, J. Landauer, M. Wapler \& U. Lang (Eds.), Virtual Environment '98. Wien: Springer Verlag.

Driver, J. \& Mattingley, J.B. (1998). Parietal neglect and visual awareness. Nature Neuroscience, 1, 17-22.

Duhamel, J.-R., Colby, C.L. \& Goldberg, M.E. (1992a). The updating of the representation of visual space in parietal cortex by intended eye movements. Science, 255, 90-92.

Duhamel, J.-R., Goldberg, M.E., Fitzgibbon, E.J., Sirigu, A. \& Grafman, J. (1992b). Saccadic dysmetria in a patient with a right frontoparietal lesion. Brain, 115, 1387-1402.

Epstein, R. \& Kanwisher, N. (1998). A cortical representation of the local visual environment. Nature, 392, 598-601. 
Exner, C., Boucsein, K., Degener, D., Irle, E. \& Weniger, G. (2004). Impaired emotional learning and reduced amygdala size in schizophrenia: A 3-month follow-up. Schizophrenia Research, 71, 493-503.

Farrell, M.J. (1996). Topographical disorientation. Neurocase, 2, 509-520.

Farrer, C. \& Frith, C.D. (2002). Experiencing oneself vs. another person as being the cause of an action: The neural correlates of the experience of agency. NeuroImage, 15, 596-603.

Fleming, K., Goldberg, T.E., Binks, S., Randolph, C., Gold, J.M. \& Weinberger, D.R. (1997). Visuospatial working memory in patients with schizophrenia. Biological Psychiatry, 41, 43-49.

Flor-Henry, P. (1969). Psychosis in temporal lobe epilepsy. Epilepsia, 10, 363-395.

Foong, J., Symms, M.R., Barker, G.J., Maier, M., Woermann, F.G., Miller, D.H. \& Ron, M.A. (2001). Neuropathological abnormalities in schizophrenia: Evidence from magnetization transfer imaging. Brain, 124, 882-891.

Franck, N., Farrer, C., Georgieff, N., Marie-Candine, M., Daléry, J., d’Amato, T. \& Jeannerod, M. (2001). Defective recognition of one's own actions in patients with schizophrenia. The American Journal of Psychiatry, 158, 454-459.

Frederikse, M., Lu, A., Aylward, E., Barta, P., Sharma, T. \& Pearlson, G. (2000). Sex differences in inferior parietal lobule volume in schizophrenia. The American Journal of Psychiatry, 157, 422-427.

Frith, C.D. (1987). The positive and negative symptoms of schizophrenia reflect impairments in the perception and initiation of action. Psychological Medicine, 17, 631648.

Frith, C.D., Blakemore, S.-J. \& Wolpert, D.M. (2000). Explaining the symptoms of schizophrenia: Abnormalities in the awareness of action. Brain Research Reviews, 31, 357363.

Gillner, S. \& Mallot, H.A. (1998). Navigation and acquisition of spatial knowledge in a virtual maze. Journal of Cognitive Neuroscience, 10, 445-63.

Gilmore, R.O. \& Johnson, M.H. (1997). Body-centered representations for visually-guided action emerge during early infancy. Cognition, 65, B1-B9.

Gitelman, D.R., Nobre, A.C., Parrish, T.B., LaBar, K.S., Kim, Y.H., Meyer, J.R. \& Mesulam, M.M. (1999). A large-scale distributed network for covert spatial attention: Further anatomical delineation based on stringent behavioural and cognitive controls. Brain, 122, 1093-1106.

Glantz, K., Durlach, N.I., Barnett, R.C. \& Aviles, W.A. (1997). Virtual reality (VR) and psychotherapy: Opportunities and challenges. Presence, 6, 87-105. 
Gogtay, N., Sporn, A., Clasen, L.S., Greenstein, D., Giedd, J.N., Lelane, M., Gochman, P.A., Zijdenbos, A. \& Rapoport, J.L. (2003). Structural brain MRI abnormalities in healthy siblings of patients with childhood-onset schizophrenia. The American Journal of Psychiatry, 160, 569-571.

Goldberg, T.E. \& Weinberger, D.R. (1986). Methodological issues in the neuropsychological approach to schizophrenia. In H.A. Nasrallah \& D.R. Weinberger (Eds.), Handbook of schizophrenia, Vol. 1: The neurology of schizophrenia (chapter 6, pp. 141-156). Amsterdam: Elsevier Science Publishers.

Goldberg, E. \& Seidman, L.J. (1991). Higher cortical functions in normals and in schizophrenia: A selective review. In S.R. Steinhauer, J.H. Gruzelier \& J. Zubin (Eds.), Handbook of schizophrenia, Vol. 5: Neuropsychology, psychophysiology and information processing (chapter 21, pp. 553-597). Amsterdam: Elsevier Science Publishers.

Goldberg, M.E., Bisley, J., Powell, K.D., Gottlieb, J. \& Kusunoki, M. (2002). The role of the lateral intraparietal area of the monkey in the generation of saccades and visuospatial attention. Annals of the New York Academy of Sciences, 956, 205-15.

Goldman-Rakic, P.S. (1994). Working memory dysfunction in schizophrenia. Journal of Neuropsychiatry and Clinical Neurosciences, 6, 348-357.

Goldman-Rakic, P.S. \& Selemon, L.D. (1997). Functional and anatomical aspects of prefrontal pathology in schizophrenia. Schizophrenia Bulletin, 23, 437-458.

Goldstein, G. (1986). The neuropsychology of schizophrenia. In I. Grant \& K.M. Adams (Eds.), Neuropsychological assessment of neuropsychiatric disorders (chapter 8, pp. 147171). New York: Oxford University Press.

Goldstein, J.M. \& Tsuang, M.T. (1988). The process of subtyping schizophrenia: Strategies in the search for homogeneity. In M.T. Tsuang \& J.C. Simpson (Eds.), Handbook of schizophrenia, Vol. 3: Nosology, epidemiology and genetics (chapter 4, pp. 63-83). Amsterdam: Elsevier Science Publishers.

Goldstein, J.M., Goodmann, J.M., Seidman, L.J., Kennedy, D.N., Makris, N., Lee, H., Tourville, J., Caviness, V.S., Faraone, S.V. \& Tsuang, M.T. (1999). Cortical abnormalities in schizophrenia identified by structural magnetic resonance imaging. Archives of General Psychiatry, 56, 537-547.

Graap, K. Anderson, P, Zimand, E., Rothbaum, B.O., Hodges, L.F. \& Wilson, J. (2004). Treating fear of flying in virtual reality: A controlled study. CyberPsychology \& Behavior, 7, 285-286.

Grön, G., Wunderlich, A.P., Spitzer, M. Tomczak, R. \& Riepe, M.W. (2000). Brain activation during human navigation: Gender-different neural networks as a substrate of performance. Nature Neuroscience, 3, 404-408.

Guazzelli, A., Bota, M. \& Arbib, M.A. (2001). Competitive hebbian learning and the hippocampal place cell system: Modeling the interaction of visual and path integration cues. Hippocampus, 11, 216-39. 
Gur, R.E., Cowell, P., Turetsky, B.I., Gallacher, F., Cannon, T., Bilker, W. \& Gur, R.C. (1998). A follow-up magnetic resonance imaging study of schizophrenia. Archives of General Psychiatry, 55, 145-152.

Gur, R.E., Turetsky, B.I., Cowell, P.E., Finkelman, C., Maany, V., Grossman, R.I., Arnold, S.E., Bilker, W.B. \& Gur, R.C. (2000). Temporolimbic volume reductions in schizophrenia. Archives of General Psychiatry, 57, 769-775.

Hahlweg, K. (2000). Schizophrenie. In J. Margraf (Hrsg.), Lehrbuch der Verhaltenstherapie, Band 2 (Kapitel 17, S. 309-336). Heidelberg: Springer Verlag.

Hambrecht, M. \& Hafner, H. (1996). Substance abuse and the onset of schizophrenia. Biological Psychiatry, 40, 1155-1163.

Harris, E.C. \& Barraclough, B. (1997). Suicide as an outcome for mental disorders. The British Journal of Psychiatry, 170, 205-228.

Harrison, P.J. (1999). The neuropathology of schizophrenia. Brain, 122, 593-624.

Hartley, T., Maguire, E.A., Spiers, H.J. \& Burgess, N. (2003). The well-worn route and the path less travelled: Distinct neural bases of route following and wayfinding in humans. Neuron, 37, 877-88.

Harvey, S.A., Nelson, E., Haller, J.W. \& Early, T.S. (1993). Lateralized attentional abnormality in schizophrenia is correlated with severity of symptoms. Biological Psychiatry, 33, 93-99.

Heinrichs, R.W. \& Zakzanis, K.K. (1998). Neurocognitive deficit in schizophrenia: A quantiative review of the evidence. Neuropsychology, 12, 426-445.

Helmchen, H., Henn, F., Lauter, H. \& Sartorius, N. (Hrsg.) (2000). Psychiatrie der Gegenwart - Band 5: Schizophrene \& Affektive Störungen (4. Auflage). Berlin: Springer Verlag.

Hendrix, C. \& Barfield, W. (1996). The sense of presence within auditory virtual environments. Presence, 5, 290-301.

Ho, B.-C., Andreasen, N.C., Nopoulos, P., Arndt, S., Magnotta, V. \& Flaum, M. (2003). Progressive structural brain abnormalities and their relationship to clinical outcome. Archives of General Psychiatry, 60, 585-594.

Hogrefe, G.J. (2004). Testkatalog 2004/05. Göttingen: Hogrefe.

Höschel, K., Irle, E. \& Rüther, E. (1998). Psychopathologische Symptomatik und kognitive Leistungen schizophrener Patienten. Fortschritte der Neurologie - Psychiatrie, $66,512-519$.

Huguelet, P., Zanello, A. \& Nicastro, R. (2000). A study of visual and auditory verbal working memory in schizophrenic patients compared to healthy subjects. European Archives of Psychiatry and Clinical Neuroscience, 250, 79-85. 
Hutton, S.B., Puri, B.K., Cuncan, L.J., Robbins, T.W. \& Barnes, T.R. (1998). Executive function in first episode schizophrenia. Psychological Medicine, 28, 463-473.

Irle, E., Lange, C. \& Sachsse, U. (2005). Reduced size and abnormal asymmetry of parietal cortex in women with borderline personality disorder. Biological Psychiatry, 174, 173-182.

Jablensky, A. (2000). Symptome schizophrener Störungen. In H. Helmchen, F. Henn, H. Lauter \& N. Sartorius (Eds.), Psychiatrie der Gegenwart - Band 5: Schizophrene \& Affektive Störungen (Ausgabe 4, Kapitel 1, S. 3-53). Berlin: Springer Verlag.

Jansma, J.V., Ramsey, N.F., van der Wee, N.J.A. \& Kahn, R.S. (2004). Working memory capacity in schizophrenia: A parametric fMRI study. Schizophrenia Research, 68, 159-171.

Jeste, D.V., Gladsjo, J.A. \& Lindamer, L.A. \& Lacro, J.P. (1996). Medical comorbidity in schizophrenia. Schizophrenia Bulletin, 22, 413-430.

Jobe, T.H. \& Harrow, M. (2005). Long-term outcome of patients with schizophrenia: A review. Canadian Journal of Psychiatry, 50, 892-900.

Kaltenborn, K.F. \& Rienhoff, O. (1993). Virtual reality in medicine. Methods of Information in Medicine, 32, 407-417.

Keshavan, M.S., Sanders, R.D., Sweeney, J.A., Diwadkar, V.A., Goldstein, G., Pettegrew, J.W. \& Schooler, N.R. (2003). Diagnostic specifity and neuroanatomical validity of neurological abnormalities in first-episode psychoses. The American Journal of Psychiatry, 160, 1298-1304.

Kim, J.-J., Kwon, J.S., Park, H.J. Youn, T., Kang, D.H., Kim, M.S., Lee, D.S. \& Lee, M.C. (2003). Functional disconnection between the prefrontal and parietal cortices during working memory processing in schizophrenia: A $\left({ }^{15} \mathrm{O}\right) \mathrm{H}_{2} \mathrm{O}$ study. The American Journal of Psychiatry, 160, 919-923.

Kindermann, S.S., Brown, G.G., Zorrilla, L.E., Olsen, R.K. \& Jeste, D.V. (2004). Spatial working memory among middle-aged and older patients with schizophrenia and volunteers using fMRI. Schizophrenia Research, 68, 203-216.

Kindsmüller, M., Kaindl, A., Schuri, U. \& Zimmer, A. (1999). Topographische Orientierung bei Patienten mit erworbener Hirnschädigung. Zeitschrift für Neuropsychologie, 10, 77-86.

Kirkpatrick, B., Conley, R.R., Kakoyannis, A., Reep, R.L. \& Roberts, R.C. (1999). The insterstitial cells of the white matter in the inferior parietal cortex in schizophrenia: An unbiased cell-counting study. Synapse, 34, 95-102.

Kirkpatrick, B., Messias, N.C., Conley, R.R. \& Roberts, R.C. (2003). Interstitial cells of the white matter in the dorsolateral prefrontal cortex in deficit and nodeficit schizophrenia. The Journal of Nervous and Mental Disease, 191, 563-567. 
Kishimoto, H., Kuwahara, H., Ohno, S., Takazu, O., Hama, Y., Sato, C., Ishii, T., Nomura, Y., Fujita, H., Miyauchi, T., Matsushita, M., Yokoi, S. \& Iio, M. (1987). Three subtypes of chronic schizophrenia identified using 11-C-glucose positron emission tomography. Psychiatry Research, 21, 285-292.

Kitchin, R.M. (1994). Cognitive maps: What are they and why study them? Journal of Environmental Psychology, 14, 1-19.

Klinger, E., Chemin, I., Hübner, R. \& Marié, M. (2004). A virtual supermarket to assess cognitive planning. CyberPsychology \& Behavior, 7, 292-293.

Kolb, B. \& Whishaw, I.Q. (1996). Neuropsychologie (2. Auflage). Heidelberg: Spektrum Akademischer Verlag.

Königsberg, H.W., Buchsbaum, M.S., Buchsbaum, B.R., Schneiderman, J.S., Tang, C.Y., New, A., Goodman, M. \& Seiver, L.J. (2005). Functional MRI of visuospatial working memory in schizotypal personality disorder: A region-of-interest analysis. Psychological Medicine, 35, 1019-1030.

Kottlow, M., Stoermer, R., Mager, R., Mueller-Spahn, F., Amditis, A. \& Bullinger, A.H. (2004). Development and Evaluation of a modular psychophysiological test battery for use with virtual environments/augmented reality applications. CyberPsychology \& Behavior, 7, 274-275.

Kraepelin, E. (1919): Dementia praecox. New York: Churchill Livingstone Inc..

Krüger, H.P., Körner, Y., Buld, S., Hoffmann, S., Flöthe, J. Tietze, H., Naumann, M. \& Merz, B. (2005). Fahrtauglichkeit und M. Parkinson. Medical Reporter, 3, 11.

Ku, J., Cho, W., Kim, J.J., Peled, A., Wiederhold, B.K., Wiederhold, M.D, Kim, I.Y. Lee, J.H. \& Kim, S.I. (2003). A virtual environment for investigating schizophrenic patients' characteristics: Assessment of cognitive and navigation ability. CyberPsychology, 6, $397-$ 404.

Kubicki, M., Shenton, M.E., Salisbury, D.F., Kasai, H.K., Kikinis, R., Jolesz, F.A. \& McCarley, R.W. (2002). Voxel-based morphometric analysis of gray matter in first episode schizophrenia. NeuroImage, 17, 1711-1719.

Kurtz, M., Pearlson, G. \& Astur, R.S. (2004). Investigation of driving behaviour in individuals with schizophrenia using a VR driving simulator. CyberPsychology \& Behavior, 7, 308-309.

Lampton, D.R., Knerr, B.W., Goldberg, S.L., Bliss, J.P., Moshell, J.M. \& Blau, B.S. (1994). The Virtual Environment Performance Assessment Battery (VEPAB): Development and evaluation. Presence, 3, 145-157.

Lange, C. (2003). Neuropsychologische Testleistungen, psychopathologische Symptomatik und Hippocampusvolumen bei psychisch traumatisierten Patientinnen. Dissertation. Göttingen: Universität Göttingen, Fachbereich Neuropsychologie und Psychopathologie. 
Lawrie, S.M. \& Abukmeil, S. (1998). Brain abnormality in schizophrenia. The British Journal of Psychiatry, 172, 110-120.

Lee, J.H., Hahn, W.Y., Kim, H.S., Ku, J.H., Park, D.W., Kim, S.H. Yang, B.H., Lim, Y.S. \& Kim, S.I. (2004). A functional magnetic resonance imaging (fMRI) study of nicotine craving and cue exposure therapy (CET) by using visual stimuli. CyberPsychology \& Behavior, 7, 290.

Levin, S. \& Yurgelun-Todd, D. (1989). Contributions of clinical neuropsychology to the study of schizophrenia. Journal of Abnormal Psychology, 98, 341-356.

Levine, D., Warach, J. \& Farah, M. (1985). Two visual systems in mental imagery: Dissociation of what and where in imagery disorders due to bilateral posterior cerebral lesions. Neurology, 35, 1010-1018.

Lezak, M.D. (1995). Neuropsychological Assessment (3rd edition). Oxford: Oxford University Press.

Liddle, P.F. (1987). The symptoms of chronic schizophrenia. The British Journal of Psychiatry, 151, 145-151.

Lieberman, J.A., Tollefson, G.D., Charles, C., Zipursky, R., Sharma, T., Kahn, R.S., Keefe, R.S.E., Green, A.I., Gur, R.E., McEvoy, J., Perkins, D., Hamer, R.M., Gu, H. \& Tohen, M. (2005). Antipsychotic drug effects on brain morphology in first-episode psychosis. Archives of General Psychiatry, 62, 361-370.

MacDonald, P.A. \& Paus, T. (2003). The role of parietal cortex in awareness of selfgenerated movements: A transcranial magnetic stimulation study. Cerebral Cortex, 13, 962-967.

Maguire, E.A., Burke, T., Phillips, J. \& Staunton, H. (1996). Topographical disorientation following unilateral temporal lobe lesions in humans. Neuropsychologia, 34, 993-1001.

Maguire, E.A., Fackowiak, R.S.J., Frith, C.D. (1997). Recalling routes around london: Activation of the right hippocampus in taxi drivers. The Journal of Neuroscience, 17, 7103-7110.

Maguire, E.A., Burgess, N., Donnett, J.G., Frackowiak, R.S.J., Frith, C.D. \& O’Keefe, J. (1998a). Knowing where and getting there: A human navigation network. Science, 280, 921-924.

Maguire, E.A., Frith, C.D., Burgess, N., Donnett, J.G. \& O’Keefe, J. (1998b). Knowing where things are: Parahippocampal involvement in encoding object locations in virtual large scale space. Journal of Cognitive Neuroscience, 10, 71-76.

Marsh, L., Lim, K., Hoff, A., Harris, D., Beal, M., Minn, K., Faustman, W., Csernansky, J., Sullivan, E. \& Pfefferbaum, A. (1997). Structural MRI abnormalities in schizophrenia: A comparison of state hospital and veterans hospital patients. Schizophrenia Research, 24, 152. 
Marsh, L., Lim, K.O., Hoff, A.L., Harris, D., Beal, M., Minn, K., Faustman, W.O., Csernansky, J.G., Sullivan, E.V. \& Pfefferbaum, A. (1999). Severity of schizophrenia and magnetic resonance imaging abnormalities: A comparison of state and veterans hospital patients. Biological Psychiatry, 45, 49-61.

Marshall, J.W.B., Baker, H.F. \& Ridley, R.M. (2002). Contralesional neglect in monkeys with small unilateral parietal cortical ablations. Behavioural Brain Research, 136, 257-265.

Märtens, K. (2002). Visuell-räumliche Informationsverarbeitungsleistungen in einem "virtual reality"-gestützten Navigationsaufgabe bei Patienten mit Mediaischämie. Unveröffentlichte Diplomarbeit. Göttingen: Universität Göttingen, Fachbereich Neuropsychologie und Psychopathologie.

Maruff, P., Hay, D., Malone, V. \& Currie, J. (1995). Asymmetries in the covert orienting of visual spatial attention in schizophrenia. Neuropsychologia, 33, 1205-1223.

Maruff, P., Wood, S.J., Velakoulis, D., Smith, D.J., Soulsby, B., Suckling, J., Bullmore, E.T. \& Pantelis, C. (2005). Reduced volume of parietal and frontal association areas in patients with schizophrenia characterized by passivity delusions. Psychological Medicine, $35,783-789$.

McCarley, R.W., Wible, C.G., Frumin, M., Hirayashu, Y., Levitt, J.J., Fischer, I.A. \& Shenton, M.E. (1999). MRI anatomy of schizophrenia. Biological Psychiatry, 45, 10991119.

McGhie, A. \& Chapman, J. (1961). Disorders of attention and perception in early schizophrenia. British Journal of Medical Psychology, 34, 103-116.

McGilchrist, I. (1993). Inferior parietal gray matter loss in schizophrenia. 146th Annual Meeting of the American Psychiatric Association, San Francisco, New Research Abstract 230: 117 .

McGuire, P.K., Silbersweig, D.A., Wright, I., Murray, R.M., Frackowiak, R.S.J. \& Frith, C.D. (1996). The neural correlates of inner speech and auditory verbal imagery in schizophrenia: Relationship to auditory verbal hallucinations. The British Journal of Psychiatry, 169, 148-159.

Mehlitz, M., Weniger, G., Kleinoeder, T. \& Rienhoff, O. (1998). Virtual Reality in der medizinischen Anwendung - Perspektiven für die Krankenversorgung. Das Krankenhaus, 8, 463-8.

Mehlitz, M.A. (2004). Aufbau eines medizinischen Virtual-Reality-Labors und Entwicklung eines VR-gestützten neuropsychologischen Testsystems mit einer präklinischen und klinischen Evaluationsstudie. Dissertation. Göttingen: Universität Göttingen, Fachbereich Statistik und Informatik der Medizinischen Fakultät.

Mellet, E., Tzourio, N., Crivello, F., Joliot, M. Denis, M. \& Mazoyer, B. (1996). Functional anatomy of spatial mental imagery generated from verbal instructions. The Journal of Neuroscience, 16, 6504-6512. 
Mellet, E., Bricogne, S. Tzourio-Mazoyer, N., Ghaem, O. Petit, L., Zago, L., Etard, O., Berthoz, A., Mazoyer, B. \& Denis, M. (2000a). Neural correlates of mental exploration: The impact of route versus survey perspective learning.NeuroImage, 12, 588-600.

Mellet, E., Kosslyn, S.M., Tzourio,Mazoyer, N., Bricogne, S., Denis, M. \& Mazoyer, B. (2000b). Functional anatomy of high-resolution visual mental imagery. Journal of Cognitive Neuroscience, 12, 98-109.

Menon, R.R., Barta, P.E., Aylward, E.H., Richards, S.S., Vaughn, D.D., Tien, A.Y., Harris, G.J. \& Pearlson, G.D. (1995). Posterior superor temporal Gyrus in schizophrenia: Grey matter changes and clinical correlates. Schizophrenia Research, 16, 127-135.

Mesulam, M.-M. (1985). Patterns in behavioral neuroanatomy: Association areas, the limbic system, and hemispheric specialization. In M.-M. Mesulam, Principles of behavioral neurology (chapter 1, pp. 1-70). Philadelphia: F.A. Davis Company.

Mesulam, M.-M. (1990). Large-scale neurocognitive networks and distributed processing for attention, language and memory. Annals of Neurology, 28, 597-613.

Mesulam, M.-M. (1998). From sensation to cognition. Brain, 121, 1013-1052.

Mishkin, M., Ungerleider, L.G. \& Macko, K.A. (1983). Object vision and spatial vision: Two cortical visual pathways. Trends in Neurosciences, 6, 414-417.

Mitelman, S.A., Shihabuddin, L., Brickman, A.M., Hazlett, E.A. \& Buchsbaum, M.S. (2003). MRI assessment of gray and white matter distribution in broadmann's areas of the cortex in patients with good and poor outcomes. The American Journal of Psychiatry, 160, 2154-2168.

Moser, E., Moser, M.-B. \& Andersen, P. (1993). Spatial learning impairment parallels the magnitude of dorsal hippocampal lesions, but is hardly present following ventral lesions. The Journal of Neuroscience, 13, 3916-3925.

Mühlberger, A., Herrmann, M.J., Wiedemann, G., Ellgring, H. \& Pauli, P. (2001). Repeated exposure of flight phobics to flights in virtual reality. Behaviour Research and Therapy, 39, 1033-1050.

Mühlberger, A., Petrusek, S., Herrmann, M. \& Pauli, P. (2005). Biocyperpsychologie: Subjektive und physiologische Reaktionen von Flugphobikern und Gesunden bei Exposition mit virtuellen Flügen. Zeitschrift für Klinische Psychologie und Psychotherapie, 34, 133-143.

Nelson, H.E. (1982). National Adult Reading Test (NART): Test Manual. Windsor, UK: NFER Nelson.

Nelson, M.D., Saykin, A.J., Flashman, L.A. \& Riordan, H.J. (1998). Hippocampal volume reduction in schizophrenia as assessed by magnetic resonance imaging. Archives of General Psychiatry, 55, 433-440.

Nieuwenhuys, R., Voogd, J. \& van Huijzen, C. (1978). Das Zentralnervensystem des Menschen. Berlin: Springer Verlag. 
Niznikiewicz, M., Donnino, R., McCarley, R.W., Nestor, P.G., Iosifescu, D.V., O`Donnell, B., Levitt, J. \& Shenton, M.E. (2000). Abnormal angular Gyrus asymmetry in schizophrenia. The American Journal of Psychiatry, 157, 428-437.

Nobre, A.C., Gitelman, D.R., Dias, E.C. \& Mesulam, M.M. (2000). Covert visual spatial orienting and saccades: Overlapping neural systems. NeuroImage, 11, 210-216.

O’Carroll, R. (2000). Cognitive impairment in schizophrenia. Advances in Psychiatric Treatment, 6, 161-168.

O’Donnell, B.F., Swearer, J.M, Smith, L.T., Nestor, P.G., Shenton, M.E., McCarley, R.W. (1996). Selective deficits in visual perception and recognition in schizophrenia. The American Journal of Psychiatry, 153, 687-692.

O’Keefe, J. \& Nadel, L. (1978). The hippocampus as a cognitive map. Oxford: Clarendon Press.

Onitsuka, T., Shenton, M.E., Salisbury, D.F., Dickey, C.C., Kasai, K., Toner, S.K., Frumin, M. Kikinis, R., Jolesz, F.A. \& McCarley, R.W. (2004). Middle and inferior temporal Gyrus gray matter volume abnormalities in chronic schizophrenia: An MRI study. The American Journal of Psychiatry, 161, 1603-1611.

Overall, J.E. \& Gorham, D.R. (1962). The Brief Psychiatric Rating Scale (BPRS). Psychiatric Reports, 10, 799-812.

Palmer, B. (2005). The lifetime risk of suicide in schizophrenia: A reexamination. Archives of General Psychiatry, 62, 247-253.

Pantelis, C., Velakoulis, D., McGorry, D., Wood, S.J., Suckling, J., Phillips, L.J., Yung, A.R., Bullmore, E.T., Brewer, W., Soulsby, B., Desmond, P. \& McGuire, P.K. (2003). Neuroanatomical abnormalities before and after onset of psychosis: A cross-sectional and longitudinal MRI comparison. The Lancet, 361, 281-288.

Park, S. \& Holzman, P.S. (1992). Schizophrenics show spatial working memory deficits. Archives of General Psychiatry, 49, 975-982.

Park, S., Holzman, P.S. \& Goldman-Rakic, P.S. (1995). Spatial working memory deficits in the relatives of schizophrenic patients. Archives of General Psychiatry, 52, 821-828.

Parrott, M., Bowman, D. \& Ollendick, T. (2004). An immersive virtual environment for the treatment of ophidiophobia. CyberPsychology \& Behavior, 7, 301-302.

Paulus, M.P., Hozak, N.E., Zauscher, B.E., Frank, L., Brown, G.G., McDowell, J. \& Braff, D.L. (2002). Parietal dysfunction is associated with increased outcome-related decisionmaking in schizophrenia patients. Biological Psychiatry, 51, 995-1004.

Pausch, R., Crea, T. \& Conway, M. (1992). A literature survey for virtual environments: Military flight simulator visual systems and simulator sickness. Presence, 1, 344-363. 
Payne, J., Malla, A., Norman, R., Windell, D. \& Brown, N. (2006). Status of first-episode psychosis patients presenting for routine care in a defined catchment area. Canadian Journal of Psychiatry, 51, 42-47.

Pearlson, G.D., Petty, R.G., Ross, C.A. \& Tien, A.Y. (1996). Schizophrenia: A disease of heteromodal association cortex? Neuropsychopharmacology, 14, 1-17.

Pearlson, G.D., Barta, P.E., Powers, R.E., Menon, R.R., Richards, S.S., Aylward, E.H., Federman, E.B., Chase, G.A., Petty, R.G. \& Tien, A.Y. (1997). Medial and superior temporal gyral volumes and cerebral asymmetry in schizophrenia versus bipolar disorder. Biological Psychiatry, 41, 1-14.

Pearlson, G.D. \& Marsh, L. (1999). Structural brain imaging in schizophrenia: A selective review. Biological Psychiatry, 46, 627-649.

Pearlson, G.D. (2000). Neurobiology of schizophrenia. Annals of Neurology, 48, 556-566.

Peled, A. (1999). Multiple constraint organization in the brain: A theory for schizophrenia. Brain Research Bulletin, 49, 245-250.

Penfield, W. \& Perot, P. (1963). The bain's record of auditory and visual experience. Brain, 86, 595-705.

Peralta, V., Cuesta, M.J. \& de Leon, J. (1994). An empirical analysis of latent structures underlying schizophrenic symptoms: A four-syndrome model. Biological Psychiatry, 36, 726-736.

Petersen, S.E., Robinsons, D.L. \& Currie, J.N. (1989). Influences of lesions of parietal cortex on visual spatial attention in humans. Experimental Brain Research, 76, 267-280.

Petty, R.G., Barta, P.E., Pearlson, G.D., McGilchrist, I.K., Lewis, R.W., Tien, A.Y., Pulver, A., Vaughn, D.D., Casanova, M.F. \& Powers, R.E. (1995). Reversal of asymmetry of the planum temporale in schizophrenia. The American Journal of Psychiatry, 152, 715721.

Pimentel, K. \& Teixeira, K. (1995). Virtual Reality - Through the new looking glasses. New York: McGraw Hill.

Pol, H.E.H., Schnack, H.G., Mandl, R.C.W., van Haren, N.E.M., Koning, H., Collins, L., Evans, A.C. \& Kahn, R.S. (2001). Focal gray matter density changes in schizophrenia. Archives of General Psychiatry, 58, 1118-1125.

Pompili, M., Ruberto, A., Kotzalidis, G.D., Girardi, P. \& Tatarelli, R. (2004). Suicide and awareness of illness: An overview. Bulletin of the Menninger Clinic, 68, 297-318.

Posner, M.I. (1980). Orienting of attention. Quaterly Journal of Experimental Psychology, 32, 3-25.

Posner, M.I. \& Petersen, S.E. (1990). The attention system of the human brain. Annual Review of Neuroscience, 13, 25-42. 
Pugnetti, L., Mendozzi, L., Motta, A., Cattaneo, A., Barbieri, E. \& Brancotti, A. (1995). Evaluation and retraining of adults' cognitive impairments: Which role for virtual reality technology? Computers in Biology and Medicine, 25, 213-227.

Quintana, J., Wong, T., Ortiz-Portillo, E., Kovalik, E., Davidson, T., Marder, S.R. \& Mazziotta, J.C. (2003). Prefrontal-posterior parietal networks in schizophrenia: Primary dysfunctions and secondary compensations. Biological Psychiatry, 53, 12-24.

Rademacher, J., Galaburda, A.M., Kennedy, D.N., Filipek, P.A. \& Caviness, V.S. (1992). Human cerebral cortex: Location, parcellation and morphometry with magnetic resonance imaging. Journal of Cognitive Neuroscience, 4, 352-374.

Raja, P. (1996). Neuroanalytical correlates of the positive and negative symptoms of schizophrenia. Dissertation Abstracts International: Section B: Sciences and Engineering, 57, 4083.

Rajarethinam, R., DeQuardo, J.R., Miedler, J., Arndt, S., Kirbat, R., Brunberg, J.A. \& Tandon, R. (2001). Hippocampus and amygdala in schizophrenia: Assessment of the relationship of neuroanatomy to psychopathology. Psychiatric Research: Neuroimaging. 108, 79-87.

Raz, S. \& Raz, N. (1990). Structural brain abnormalities in the major psychoses: A quantitative review of the evidence from computerized imaging. Psychological Bulletin, 108, 93-108.

Reitan, R.M. (1992). Trail Making Test. Manual for administration and scoring. South Tucson, AZ: Reitan Neuropsychological Laboratory.

Reite, M., Sheeder, J., Teale, P., Adams, M., Richardson, D., Simon, J., Jones, R.H. \& Rojas, D.C. (1997). Magnetic source imaging evidence of sex differences in cerebral lateralization in schizophrenia. Archives of General Psychiatry, 54, 433-440.

Roob, K. (2002). Beziehung zwischen morphologischen Hirnveränderungen und neuropsychologischen Defiziten bei schizophrenen Patienten. Dissertation. Jena: Universität Jena, Fachbereich Psychologie.

Ross, C. \& Pearlson, G.D. (1996). Schizophrenia, the heteromodal association neocortex and development - potential for a neurogenetic approach. Trends in Neuroscience, 19, 171-176.

Rothbaum, B.O., Hodges, L.F., Kooper, R., Opdyke, D., Williford, J. \& North, M.M. (1995). Effectiveness of virtual reality graded exposure in the treatment of acrophobia. The American Journal of Psychiatry, 152, 626-628.

Rothbaum, B.O. Hodges, L.F., Watson, B.A., Kessler, G.D. \& Opdyke, D. (1996). Virtual reality exposure therapy in the treatment of fear of flying: A case report. Behaviour Research and Therapy, 34, 477-481.

Ruddle, R.A., Payne, S.J. \& Jones, D.M. (1997). Navigating buildings in „desk-top“ virtual environments: Experimental investigations using extended navigational experience. Journal of Experimental Psychology: Applied, 3, 143-159. 
Ruddle, R.A., Payne, S.J. \& Jones, D.M. (1998). Navigating large-scale „desk-top“ virtual buildings: Effects of orientation aids and familarity. Presence, 7, 179-192.

Rund, B.R. (1983). The effect of distraction on focal attention in paranoid and nonparanoid schizophrenic patients compared to normals and non-psychotic psychiatric patients. Journal of Psychiatric Research, 17, 241-250.

Rund, B.R. (1998). A review of longitudinal studies of cognitive functions in schizophrenia patients. Schizophrenia Bulletin, 24, 425-435.

Salgado-Pineda, P., Baeza, I., Pérez-Gómez, M., Vendrell, P., Junqué, C., Bargalló, N. \& Bernardo, M. (2003). Sustained attention impairment correlates to gray matter decrease in first episode neuroleptic-naïve schizophrenic patients. NeuroImage, 19, 365-375.

Sanfilipo, M., Lafargue, T., Rusinek, H., Arena, L., Loneragan, C., Lautin, A., Rotrosen, J. \& Wolkin, A. (2002). Cognitive performance in schizophrenia: Relationship to regional brain volumes and psychiatric symptoms. Psychiatry Research: Neuroimaging, 116, 1-23.

Save, E. \& Poucet, B. (2000). Hippocampal-parietal cortical interactions in spatial cognition. Hippocampus, 10, 491-499.

Saykin, A.J., Gur, R.C., Gur, R.E., Mozley, D., Mozley, L.H., Resnick, S.M., Kester, D.B. \& Stafiniak, P. (1991). Neuropsychological function in schizophrenia. Archives of General Psychiatry, 48, 618-624.

Saykin, A.J., Shtasel, D.L., Gur, R.E., Kester, D.B., Mozley, L.H., Stafiniak, P. \& Gur, R.C. (1994). Neuropsychological deficits in neuroleptic naïve patients with first-episode schizophrenia. Archives of General Psychiatry, 51, 124-131.

Schlaepfer, T.E., Harris, G.J., Tien, A.Y., Peng, L.W., Lee, S., Federman, E.B., Chase, G.A., Barta, P.E. \& Pearlson, G.D. (1994). Decreased regional cortical gray matter volume in schizophrenia. The American Journal of Psychiatry, 151, 842-848.

Schultz, S.K., O’Leary, D.S., Boles Ponto, L.L., Arndt, S., Magnotta, V., Watkins, G.L., Hichwa, R.D. \& Andreasen, N.C. (2002). Age and regional cerebral blood flow in schizophrenia: Age effects and anterior cingulated, frontal and parietal cortex. Journal of Neuropsychiatry and Clinical Neurosciences, 14, 19-24.

Seltzer, J., Conrad, C. \& Cassens, G. (1997). Neuropsychological profiles in schizophrenia: Paranoid versus undifferentiated distinctions. Schizophrenia Research, 23, 131-138.

Shapleske, J., Rossell, S.L., Chitnis, X.A., Suckling, J., Simmons, A., Bullmore, E.T., Woodruff, P.W.R. \& David, A.S. (2002). A computational morphometric MRI study of schizophrenia: Effects of hallucinations. Cerebral Cortex, 12, 1331-1341.

Shelton, A.L. \& Gabrieli, J.D.E. (2002). Neural correlates of encoding space from route and survey perspectives. The Journal of Neuroscience, 22, 2711-2717.

Shenton, M.E., Dickey, C.C., Frumin, M. \& McCarley, R.W. (2001). A review of MRI findings in schizophrenia. Schizophrenia Research, 49, 1-52. 
Shergill, S.S., Cameron, L.A., Brammer, M.J., Williams, S.C.R., Murray, R.M. \& McGuire, P.K. (2001). Modality specific neural correlates of auditory and somatic hallucinations. Journal of Neurology, Neurosurgery and Psychiatry, 71, 688-690.

Sheridan, T.B. (1992). Musings on telepresence and virtual presence. Presence: Teleoperators and Virtual Environments, 1, 120-126.

Sigmundsson, T., Suckling, J., Maier, M., Williams, S.C.R., Bullmore, E.T., Greenwood, K.E., Fukuda, R., Ron, M.A. \& Toone, B.K. (2001). Structural abnormalities in frontal, temporal and limbic regions and interconnecting white matter tracts in schizophrenic patients with prominent negative symptoms. The American Journal of Psychiatry, 158, 234-243.

Skelton, R.W., Bukach, C.M., Laurance, H.E., Thomas, K.G.F. \& Jacobs, W.J. (2000). Humans with traumatic brain injuries show place-learning deficits in computer-generated virtual space. Journal of Clinical and Experimental Neuropsychology, 22, 157-75.

Slater, M., Usoh, M. \& Steed, A. (1994). Depth of presence in virtuel environments. Presence, 3, 130-144.

Slater, M. \& Wilbur, S. (1997). A framework for immersive virtual environments (FIVE): Speculations on the role of presence in virtual environments. Presence, 6, 603-616.

Slewa-Younan, S., Gordon, E., Harris, A.W., Haig, A.R., Brown, K.J., Flor-Henry, P. \& Williams, L.M. (2004). Sex differences in functional connectivity in first-episode and chronic schizophrenia patients. The American Journal of Psychiatry, 161, 1595-1602.

Snitz, B.E., MacDonald, A.W. \& Carter, C.S. (2005). Cognitive deficits in unaffected firstdegree relatives of schizophrenia patients: A meta-analytic review of putative endophenotypes. Schizophrenia Bulletin, 32, 179-194.

Snyder, L.H., Grieve, K.L., Brotchie, P. \& Andersen, R.A. (1998). Separate body- and world-referenced representations of visual space in parietal cortex. Nature, 394, 887-891.

Spence, S.A., Brooks, D., Hirsch, S.R., Liddle, O.F., Meehan, J. \& Grasby, P.M. (1997). A PET study of voluntary movement in schizophrenic patients experiencing passivity phenomena (delusions of alien control). Brain, 120, 1997-2011.

Spiers, H.J., Burgess, N., Maguire, E.A., Baxendale, S.A., Hartley, T., Thompson, P.J. \& O’Keefe, J. (2001). Unilateral temporal lobectomy patients show lateralized topographical and episodic memory deficits in a virtual town. Brain, 124, 2476-2489.

Stark, M., Coslett, H.B. \& Saffran, E.M. (1996). Impairment of an egocentric map of locations: Implications for perception and action. Cognitive Neuropsychology, 13, 481523.

St-Jaques, J., Bouchard, S. \& Renaud, P. (2004). Long-term effectiveness of VR exposure for phobic children. CyberPsychology \& Behavior, 7, 309-310. 
Streit, M., Ioannides, A., Sinnemann, T., Wölwer, W., Dammers, J., Zilles, K. \&Gaebel, W. (2001). Disturbed facial affect recognition in patients with schizophrenia associated with hypoactivity in distributed brain regions: A magnetoencephalographic study. The American Journal of Psychiatry, 158, 1429-1436.

Sullivan, E.V., Mathalon, D.H., Lim, K.O., Marsh, L. \& Pfefferbaum, A. (1998). Patterns of regional cortical dysmorphology distinguishing schizophrenia and chronic alcoholism. Biological Psychiatry, 43, 118-131.

Sweatt, J.D. (2004). Hippocampal function in cognition. Psychopharmacology, 174, 99110 .

Sweeney, J.A., Haas, G.L., Keilp, J.G. \& Long, M. (1991). Evaluation of the stability of neuropsychological functioning after acute episodes of schizophrenia: One-year follow-up study. Psychiatry Research, 38, 63-76.

Sweet, R.A., Pierri, J.N., Auh, S., Sampson, A.R. \& Lewis, D.A. (2003). Reduced pyramidal cell somal volume in auditory association cortex of subjects with schizophrenia. Neuropsychopharmacology, 28, 599-609.

Szeszko, P.R., Strous, R.D., Goldman, R.S., Ashtari, M., Knuth, K.H., Lieberman, J.A. \& Bilder, R.M. (2002). Neuropsychological correlates of hippocampal volumes in patients experiencing a first episode of schizophrenia. The American Journal of Psychiatry, 159, 217-226.

Szeszko, P.R., Goldberg, E., Gunduz-Bruce, H., Ashtari, M., Robinson, D., Malhotra, A.K., Lencz, T., Bates, J., Crandall, D.T., Kane, J.M. \& Bilder, R.M. (2003). Smaller anterior hippocampal formation volume in antipsychotic-naïve patients with first-episode schizophrenia. The American Journal of Psychiatry, 160, 2190-2197.

Talairach, J. \& Tournoux, P. (1988). Co-planar stereotaxic atlas of the human brain. Stuttgart: Georg Thieme Verlag.

Tamminga, C.A., Thaker, G.K., Buchanan, R., Kirkpatrick, B., Alphs, L.D., Chase, T.N. \& Carpenter, T. (1992). Limbic system abnormalities identified in schizophrenia using positron emission tomography with fluordeoxyglucose and neocortical alterations with deficit syndrome. Archives of General Psychiatry, 49, 522-530.

Tan, H.-Y., Choo, W.-C., Fones, C.S.L. \& Chee, M.W.L. (2005). FMRI study of maintenance and manipulation processes within working memory in first-episode schizophrenia. The American Journal of Psychiatry, 162, 1849-1858.

Tek, C., Gold, J., Blaxton, T., Wilk, C., McMahon, R.P. \& Buchanan, R.W. (2002). Visual perceptual and working memory impairments in schizophrenia. Archives of General Psychiatry, 59, 146-153.

Tendolkar, I., Ruhrmann, S., Brockhaus-Dumke, A., Pauli, M., Mueller, R., Pukrop, R. \& Klosterkötter, J. (2005). Neural correlates of visuo-spatial attention during an antisaccade task in schizophrenia: An ERP study. International Journal of Neuroscience, 115, 681698. 
Tewes, U. (1991). HAWIE-R- Hamburg-Wechsler-Intelligenztest für Erwachsene-Revision 1991. Bern: Huber.

Thompson, P.M., Vidal, C., Giedd, J.N., Gochman, P., Blumenthal, J., Nicolson, R., Toga, A.W. \& Rapoport, J.L. (2001). Mapping adolescent brain change reveals dynamic wave of accelerated gray matter loss in very early-onset schizophrenia. Proceedings of the National Academy of Sciences of the United States of America, 98, 11650-11655.

Thorndyke, P.W. \& Hayes-Roth, B. (1982). Differences in spatial knowledge acquired from maps and navigation. Cognitive Psychology, 14, 560-589.

Tolman, E.C., Ritchie, B.F. \& Kalish, D. (1946). Studies in spatial learning: II. Place learning versus response learning. Journal of Experimental Psychology: General, 36, 221229.

Tolman, E.C., Ritchie, B.F. \& Kalish, D. (1947). Studies in spatial learning: V. Response versus place learning by the noncorrection method. Journal of Experimental Psychology: General, 37, 285-292.

Tolman, E.C. (1948). Cognitive maps in rats and men. Psychological Review, 55, 189-208.

Turetsky, B., Cowell, P.E., Gur, R.C., Grossmann, R.I., Shtasel, D.L. \& Gur, R.E. (1995). Frontal and temporal lobe brain volumes in schizophrenia. Archives of General Psychiatry, 52, 1061-1071.

Ungerleider, L.G. \& Haxby, J.V. (1994). „What“ and „where“ in the human brain. Current Opinion in Neurobiology, 4, 157-65.

Van Beilen, M., Zomeren, E.H., van den Bosch, R.J., Withaar, F. \& Bouma, A. (2005). Measuring the executive functions in schizophrenia: The voluntary allocation of effort. Journal of Psychiatric Research, 39, 585-593.

Van Veen, H.A.H.C., Distler, H.K., Braun, S.J. \& Bülthoff, H.H: (1998). Navigating through a virtual city: Using virtual reality technology to study human action and perception. Technical Report, 57, 1-11.

Vargas, J.A. (2006). Virtual Reality prepares soldiers for real war. Online-Ressource: http://www.washingtonpost.com/wp-yn/content/article/2006/02/13/AR2006021302437.html

Velakoulis, D., Stuart, G.W., Wood, S.J., Smith, D.J., Brewer, W.J., Desmond, P., Singh, B., Copolov, D. \& Pantelis, C. (2001). Selecitve bilateral hippocampal volume loss in chronic schizophrenia. Biological Psychiatry, 50, 531-539.

Waller, D., Hunt, E. \& Knapp, D. (1998). The transfer of spatial knowledge in virtual environment training. Presence, 7, 129-143.

Watzl, H. \& Rist, F. (1997). Schizophrenie. In K.Hahlweg \& A.Ehlers (Hrsg.), Psychische Störungen und ihre Behandlungen (Kapitel 1, S. 1-154). Göttingen: Hogrefe.

Wechsler, D. (1981). WAIS-R Manual. Wechsler Adult Intelligence Scale-Revised. New York: 
Wechsler, D. (1987). WMS-R-Wechsler-Memory-Scale-Revised. San Antonio: Psychological Corporation.

Weiller, C., Juptner, M., Fellows, S., Rijntjes, M., Leonhardt, G., Kiebel, S., Muller, S., Diener, H.C. \& Thilmann, A.F. (1996). Brain representation of active and passive movements. NeuroImage, 5, 105-110.

Weinberger, D.R., Berman, K.F., Suddath, R. \& Torrey, E.F. (1992). Evidence of dysfunction of a prefrontal-limbic network in schizophrenia: A magnetic resonance imaging and regional blood flow study of discordant monozygotic twins. The American Journal of Psychiatry, 149, 890-897.

Welch, R., Blackmon, T.T., Liu, A., Mellers, B.A. \& Stark, L.W. (1996). The effects of pictorial realism, delay of visual feedback, and observer interactivity on the subjective sense of presence. Presence, 5, 263-273.

Weniger, G., Exner, C. \& Irle, E. (2004a). Dissoziation von explizitem und implizitem Gedächtnis bei verschiedenen Subtypen der Schizophrenie. Posterpräsentation auf der Tagung der Deutschen Gesellschaft für Psychiatrie, Psychotherapie und Nervenheilkunde (DGPPN). Berlin.

Weniger, G., Lange, C., Rüther, E. \& Irle, E. (2004b). Differential impairments of facial affect recognition in schizophrenia subtypes and major depression. Psychiatry Research, $128,135-146$.

Weniger, G. \& Irle, E. (in press). Parahippocampal cortex lesions in the human impair egocentric navigation in a virtual environment.

Wilke, M., Kaufmann, C., Pütz, B., Wetter, T.C. \& Auer, D.P. (2001). Gray matter changes and correlates of disease severity in schizophrenia: A statistical parametric mapping study. NeuroImage, 13, 814-24.

Witmer, B.G., Bailey, J.H. \& Knerr, B.W. (1996). Virtual spaces and real world places: Transfer of route knowledge. International Journal of Human-Computer Studies, 45, $413-$ 428.

Wittchen, H.-U., Wunderlich, U., Gruschwitz, S. \& Zaudig, M. (1997). Strukturiertes Klinisches Interview für DSM-IV (SKID). Göttingen: Hogrefe.

Wojciulik, E. \& Kanwisher, N. (1999). The generality of parietal involvement in visual attention. Neuron, 23, 747-764.

Wolpert, D.M., Goodbody, S.J. \& Husain, M. (1998). Maintaining internal representations: The role of the human superior parietal lobe. Nature Neuroscience, 1, 529-533.

Wood, S.J., Proffitt, T., Mahony, K., Smith, D.J., Buchanan, J.A., Brewer, W., Stuart, G.W., Velakoulis, D., McGorry, P.D. \& Pantelis, C. (2002). Visuospatial memory and learning in first-episode schizophreniform psychosis and established schizophrenia: A functional correlate of hippocampal pathology? Psychological Medicine, 32, 429-438. 
Wright, I.C., Rabe-Hesketh, S., Woodruff, P.W.R., David, A.S., Murray, R.M. \& Bullmore, E.T. (2000). Meta-analysis of regional brain volumes in schizophrenia. The American Journal of Psychiatry, 157, 16-25.

Yang, C.R., Seamans, J.K. \& Gorelova, N. (1999). Developing a neuronal model for the pathophysiology of schizophrenia based on the nature of electrophysiological actions of dopamine in the prefrontal cortex. Neuropsychopharmacology, 21, 161-194.

Zec, R.F. \& Weinberger, D.R. (1986). Brain areas implicated in schizophrenia: A selective overview. In H.A. Nasrallah \& D.R. Weinberger (Eds.), Handbook of schizophrenia, Vol. 1: The neurology of schizophrenia (chapter 8, pp. 175-206). Amsterdam: Elsevier Science Publishers.

Zihl, J. (2004a). Neuropsychologie - Teil III: Hören, Riechen, Schmecken und Körperwahrnehmung. NeuroTransmitter, S3, 17-19.

Zihl, J. (2004b). Neuropsychologie - Teil IV: Aufmerksamkeit \& Gedächtnis. NeuroTransmitter, S3, 20-26.

Zihl, J. (2004c). Neuropsychologie - Teil V: Exekutive Funktionen. NeuroTransmitter, S3, 27-31.

Zihl, J. (2004d). Neuropsychologie - Teil VII: Neuropsychologie der Schizophrenie und Depression. NeuroTransmitter, S3, 38-40.

\section{Ergänzendes Literaturverzeichnis (Anhang)}

Banos, R., Quero, S. \& Jorquera, M. (2004). Virtual Reality as a psychological laboratory: Its utility for assessing attentional biases in anxiety disorders. CyberPsychology \& Behavior, 7, 270.

Biocca, F. (1992). Will simulation sickness slow down the diffusion ov virtual reality technology? Presence, 1, 334-343.

Bliss, J.P., Tidwell, P.D. \& Guest, M.A. (1997). The effectiveness of virtual reality for admistering spatial navigation training to firefighters. Presence, 6, 73-86.

Botella, C., Banos, R.M., Perpiná, C., Villa, H., Alcaniz, M. \& Rey, A. (1998). Virtual reality treatment of claustrophobia: A case report. Behaviour Research and Therapy, 36, 239-246.

Cobb, S.V.G., Nichols, S., Ramsey, A. \& Wilson, J.R. (1999). Virtual Reality-Induced Symptoms and Effects (VRISE). Presence, 8, 169-186.

Dumay, C.M. \& Jense, G.J. (1995). Endoscopic surgery simulation in a virtual environment. Computers in Biology and Medicine, 25, 139-148.

Fenlon, H.M., Nunes, D.P., Schroy, P.C., Barish, M.A., Clarke, P.D. \& Ferrucci, J.T. (1999). A comparison of virtual and conventional colonoscopy for the detection of colorectal polyps. The New England Journal of Medicine, 341, 1496-1503. 
Garcia-Palacios, A., Hoffman, H., See, K. (2001). Redefining therapeutic success with virtual reality exposure therapy. CyberPsychology \& Behavior, 4, 341-348.

Garcia-Palacios, A., Botella, C. Hoffman, H.G., Villa, H. \& Fabregat, S. (2004). Comparing the acceptance of VR exposure versus in-vivo exposure in a clinical sample. CyberPsychology \& Behavior, 7, 284.

Haberland, N., Kalff, R. \& Ebmeier, K. (1997). Neuronavigation in der Tumorchirurgie des zentralen Nervensystems. Onkologe, 3, 143-147.

Hamza, S., Coetzee, M., Blake, E. \& Nunez, D. (2004). A low-cost VR group support system for people living with HIV. CyberPsychology \& Behavior, 7, 276-277.

Hayes-Roth, B. Amano, K., Saker, R. Stephton, T. (2004). Online workshops training brief intervention. CyberPsychology \& Behavior, 7, 286-287.

Huang, M.P., Himle, J. \& Alessi, N.E. (2000). Vivid visualization in the experience of phobia in virtual environments: Preliminary results. CyberPsychology \& Behavior, 3, 315320 .

Kuhlen, T. \& Dohle, C. (1995). Virtual reality for physically disabled people. Computers in Biology and Medicine, 25, 205-211.

Mager, R., Stoermer, R., Estoppey, K., Mueller-Spahn, F. Amditis, A., Kottlow, M. \& Bullinger, A.H. (2004). Traffic safety investigations by means of an augmented reality driving simulation: Neurophysiological measurements in sleep-deprived subjects. CyberPsychology \& Behavior, 7, 275-276.

Müller, W.K., Ziegler, R., Bauer, A. \& Soldner, E.H. (1998). Virtual reality in surgical arthoscopic training. Journal of Image Guided Surgery, 1, 288-294.

North, M.M., North, S.M. \& Coble, J.R. (1996). Effectiveness of virtual environment desensitization in the treatment of agoraphobia. Presence, 5, 346-352.

North, M.M., North, S.M. \& Coble, J.R. (1997a). Virtual environment psychotherapy: A case study of fear of flying disorder. Presence, 6, 127-132.

North, M.M., North, S.M. \& Coble, J.R. (1997b). Virtual Reality Therapy: An Innovative paradigm. Ann Arbor, MI: IPI Press.

Ota, D., Loftin, B., Saito, T., Lea, R. \& Keller, J. (1995). Virtual reality in surgical education. Computers in Biology and Medicine, 25, 127-137.

Riva, G. (1997). The virtual environment for body image modification (VEBIM): Development and preliminary evaluation. Presence, 6, 106-117.

Riva, G., Castelnuovo, G., Gaggioli, A., Mantovani, F., Mantovani, M. \& Molinari, E. (2004). The VR-Therapy project: Free virtual reality tool for mental health therapists. CyberPsychology \& Behavior, 7, 303-304. 
Satava, R.M. (1995). Virtual Reality and Telepresence for military medicine. Computers in Biology and Medicine, 25, 229-236.

Tichon, J., Loh, J. \& King, R. (2004). Psychology student opinion of virtual reality as a tool to educate about schizophrenia. International Journal on e-learning, 3, 40-46.

Whalley, L.J. (1995). Ethical issues in the application of virtual reality to medicine. Computers in Biology and Medicine, 25, 107-14.

Winzelberg, A., Luce, K., Brown, J., Das, S., Celio, C. \& Dev, P. (2004). The use of an internet-based program to prevent eating disorders. CyberPsychology \& Behavior, 7, 311312. 


\section{Anhang}

\section{Inhaltsverzeichnis des Anhangs}

7.1. Abkürzungsverzeichnis

7.2. Versuchsmaterialien

7.2.1. Aufklärung und Einverständniserklärung $\ldots \ldots \ldots$

7.2.2. Fragebögen

7.2.2.1. Screening Fragebogen

7.2.2.2. Anamnesebogen zur Erhebung der soziodemographischen Daten _.......................

7.2.2.3. Anamnesebogen zur Erhebung ergänzender psychopathologischer Daten _ $\quad 122$

7.2.3. Klinische Fragebögen

7.2.3.1. GAF

7.2.3.2. CGI 124

7.2.3.3. BPRS

7.2.3.4. SAPS 127

7.2.3.5. SANS 129

7.2.4. Virtuelle Realität $\ldots \ldots \ldots \ldots$

7.2.4.1. Aufklärungstext VR 131

7.2.4.2. Instruktionen (via HMD präsentiert) 132

7.2.4.3. Nachbefragungsbogen VR

7.2.4.4. Rekognitionsbogen (Version: Labyrinth)

7.2.5. Magnetresonanztomographie: Aufklärungstext 137

7.3. Technische Informationen

7.3.1. VR: Technische Daten/Aufbau (PC)

7.3.2. Magnetresonanztomographie: Scanparameter und Aufnahmeeinstellungen _.........................

7.4. Ergänzende statistische Kennwerte 139

7.4.1. Ergänzung zu 4.1.3.1. (Zusammenhänge Neuropsychologie - Hirnvolumetrie) _ 139

7.4.2. Ergänzung zu 4.2.1. (Hippocampale Areale) 140

7.4.3. Ergänzung zu 4.2.2.1. (Schizophrene Subgruppen - Navigationsleistung) _........................... 141

7.5. Ergänzende inhaltliche Informationen

7.5.1. Virtuelle Realität

7.5.1.1. Komponenten eines VR-Systems

7.5.1.2. (Weitere) Vorzüge des VR-Paradigmas _........................................................

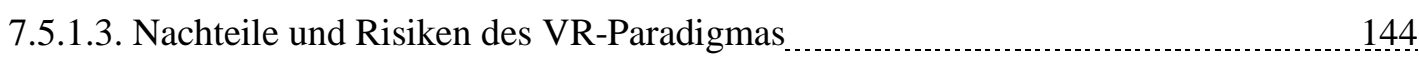

7.5.1.4. Einsatzfelder 145

7.5.1.5. Interessante Links im Internet 148

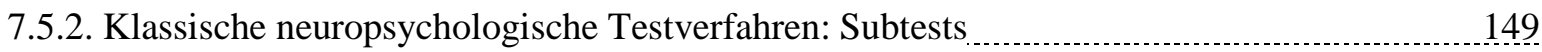




\subsection{Abkürzungsverzeichnis}

Methoden

CT Computertomographie

EEG Elektroencephalographie

EKP Ereigniskorrelierte Potentiale

HMD Head Mounted Display

MRT Magnetresonanztomographie

VR Virtuelle Realität

Cortexareale

FL

Frontallappen

(DL)PFC (dorsolateraler)präfrontaler Cortex

PL Parietallappen

IPL inferiorer Parietallappen

OL Okzipitallappen

TL Temporallappen

STG superiorer tempoler Gyrus

HASC Heteromodale Assoziationscortices

Fragebögen:

GAF Global Assessment of Functioning

CGI Clinical Global Impressions

SANS Scale for the Assessment of Negative Symptoms

SAPS Scale for the Assessment of Positive Symptoms

HAWIE-R Hamburg Wechsler Intelligenztest für Erwachsene-Revision

WMS-R Wechsler Memory Scale-Revised

TMT-A Trail Making Test Version A

TMT-B Trail Making Test Version B

Weitere:

$\begin{array}{ll}\text { FB } & \text { Fragebogen } \\ \text { IV } & \text { Informationsverarbeitung }\end{array}$




\subsection{Versuchsmaterialien}

\subsubsection{Aufklärung und Einverständniserklärung}

\section{Informationsblatt für PatientInnen und KontrollprobandInnen}

Wir haben Sie gefragt, ob Sie zur Teilnahme an einem wissenschaftlichen Forschungsprojekt bereit sind. Mit diesem Informationsblatt beschreiben wir Ihnen den Inhalt und Sinn des Forschungsprojektes.

Es ist möglich, dass bei PatientInnen, die an dieser Untersuchung teilnehmen, die hinzugewonnenen diagnostischen Informationen hilfreich für deren Therapie sind; versprechen können wir dies jedoch nicht. Wir hoffen aber, dass die Ergebnisse dieses Forschungsprojekts die zukünftige Therapie anderer PatientInnen verbessern werden.

Sie sollten an den Untersuchungen nicht teilnehmen, wenn Sie es nicht selber wollen. Wir möchten Sie ausdrücklich darauf hinweisen, dass Sie jederzeit, ohne Angabe von Gründen und ohne dass Ihnen daraus irgendwelche Nachteile entstehen, die Möglichkeit haben, Ihre Einwilligung in die Durchführung der Untersuchung zu widerrufen.

Alle Untersuchungsergebnisse, Befunde oder sonstige im Verlauf der Untersuchungen erworbenen Erkenntnisse unterstehen der ärztlichen Schweigepflicht und Vertraulichkeit. Alle personenbezogenen Daten werden gemäß den geltenden rechtlichen Bestimmungen ( $\$ 203 \mathrm{StGB}$ ) vor unbefugter Offenbarung strikt geschützt.

\section{Kurzbeschreibung des Projekts}

Wir möchten gerne erfahren, ob sich verschiedene Menschen Informationen unterschiedlich gut einprägen können und wie das Gehirn mit solchen Informationen arbeitet. Wir wissen bereits, dass Menschen, die wegen akuter psychischer Probleme behandelt werden, manchmal Probleme haben, Informationen aufzunehmen und zu behalten. Sie fühlen sich dann von ihrer Umgebung überfordert und reagieren häufig mit Rückzug. Wir möchten gerne herausfinden, inwieweit sich die Arbeitsweise des Gehirns von Menschen mit einer akuten psychischen Erkrankung von der Arbeitsweise des Gehirns von gesunden Menschen unterscheidet. Die Ergebnisse aus diesen Untersuchungen sollen uns helfen, bessere Therapiemöglichkeiten zu entwickeln, indem wir mehr über die Mechanismen psychischer Erkrankungen erfahren. 


\subsubsection{Aufklärung und Einverständniserklärung (Fortsetzung)}

\section{Einverständniserklärung}

Name:

Geb.:

wohnhaft:

Ich habe das beiliegende Informationsblatt durchgelesen. Ich fühle mich ausführlich aufgeklärt und hatte Gelegenheit, Fragen zu stellen sowie über den Sinn der Untersuchung zu sprechen. Meine Fragen sind mir zufriedenstellend beantwortet worden. Ich habe genügend Informationen zu den bei mir geplanten Untersuchungen erhalten.

Ich bin mir darüber im Klaren, dass ich jederzeit und ohne Angabe von Gründen meine Zustimmung zu der Durchführung der Untersuchung widerrufen kann und dass mir daraus keinerlei Nachteile entstehen.

Datum:

Unterschrift 


\subsubsection{Fragebögen}

\subsubsection{Screening-Fragebogen}

\section{Screening Fragen}

Jetzt möchten wir Ihnen noch einige wichtige Fragen zu Problemen stellen, die Sie möglicherweise irgendwann in Ihrem Leben gehabt haben. Bitte beantworten Sie die Fragen möglichst mit Ja oder Nein. Sollten Sie unsicher sein, sagen Sie es mir. Ich werde später im Verlauf unseres Gesprächs noch einmal genauer darauf eingehen.

1. Gab es einmal eine Zeit in Ihrem Leben, in der Sie 5 oder mehr Gläser Alkohol (Bier, Wein oder Likör) auf einmal getrunken haben?

2. Haben Sie jemals Drogen genommen?

nein unklar ja

3. Fühlten Sie sich jemals von einem ärztlich verschriebenen Medikament abhängig oder nahmen Sie mehr davon ein, als Ihnen verschrieben wurde?

3

4. Hatten Sie in Ihrem Leben einen Angstanfall, bei dem Sie ganz plötzlich in panischen Schrecken gerieten oder starke Angst hatten?

$\begin{array}{lll}1 & 2 & 3 \\ 1 & 2 & 3 \\ 1 & 2 & 3 \\ 1 & 2 & 3 \\ 1 & 2 & 3 \\ & & \\ 1 & 2 & 3 \\ 1 & 2 & 3\end{array}$

9. Ist es schon einmal vorgekommen, dass Sie Dinge immer und immer wieder tun mussten, wie z.B. sich immer wieder die Hände zu waschen oder etwas mehrmals zu kontrollieren, um sicherzugehen, dass sie es richtig gemacht haben?

10. Waren Sie in den letzten 6 Monaten besonders nervös oder ängstlich?

1

1

2 3

11. Kam es schon einmal vor, dass andere Menschen sagten, Sie seien zu dünn?

12. Hatten Sie jemals Essanfälle, bei denen Sie das Gefühl hatten, Ihr Essverhalten nicht mehr kontrollieren zu können? 
7.2.2.2. Anamnesebogen zur Erhebung der soziodemographischen Daten

VR-Projekt: ANAMNESE

1. Datum:

2. VpNr.:

3. Name, Vorname:

5. Geburtsdatum:

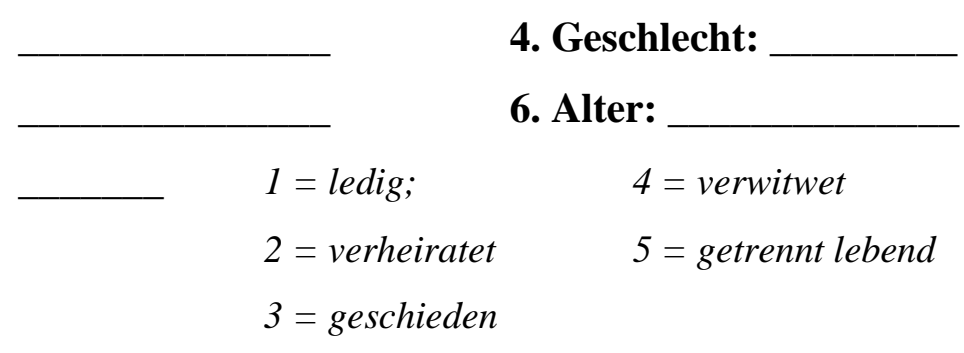

8. Körpergröße:

9. Händigkeit:

10. Schulausbildung:

$$
\begin{aligned}
& 1=\text { kein Schulabschluss } \quad 4=\text { Abitur } \\
& 2=\text { Hauptschule } \quad 5=\text { Studium } \\
& 3=\text { Realschule }
\end{aligned}
$$

11. Dauer der Schulausbildung: (in Jahren)

12. Aktuelle Berufssituation: seit:

13. Fehlsichtigkeiten:

14. Aktuelle Erkrankungen:

15. Aktuelle Medikation:

16. Vorerkrankungen (v.a. neurologisch, psychiatrisch):

17. Neurologische/psychiatrische Erkrankungen in der Familie:

18. Fremdanamnese:

19. Anmerkungen: 
7.2.2.3. Anamnesebogen zur Erhebung ergänzender psychopathologischer Daten

VR-Projekt: ANAMNESE (Patienten)

1. Vp.-Nr.:

2. Pat.-Nr.:

3. Station:

4. Behandelnder Arzt:

5. ICD-10 Diagnose(n):

6. Erstmanifestation? (Ja/Nein), wenn nein:

7. Datum der Erstmanifestation:

8. Alter des Patienten bei Erstmanifestation:

9. Anzahl vorangegangener Hospitalisierungen:

10. Medikamente:

11. Andere psychiatrische Erkrankungen:

12. Anmerkungen: 


\subsubsection{Klinische Fragebögen}

\subsubsection{GAF}

\section{DSM IV Achse V: Globale Beurteilung der Leistungsfähigkeit}

Beurteilen Sie hier die derzeitige psychische, soziale und berufliche Leistungsfähigkeit des Patienten auf einem hypothetischen Kontinuum zwischen seelischer Gesundheit und Krankheit. Beeinträchtigungen der Leistungsfähigkeit aufgrund körperlicher oder durch das soziale Umfeld bedingter Einschränkungen nicht mit einbeziehen!

90 Gute Leistungsfähigkeit auf allen Gebieten, interessiert und eingebunden in ein breites Spektrum von Aktivitäten, sozial integriert, im allgemeinen zufrieden mit dem Leben, übliche Alltagsprobleme oder Sorgen, keine oder minimale Symptome (z.B. leichte Angst vor Prüfungen, gelegentlicher Streit mit einem Familienmitglied).

80 Höchstens leichte Beeinträchtigung der sozialen, beruflichen oder schulischen Leistungsfähigkeit (z.B. zeitweiliges Zurückfallen in der Schule). Wenn Symptome vorhanden, sind diese vorübergehende oder normale Reaktionen auf psychosoziale Stressoren (z.B. Konzentrationsschwierigkeiten nach einem Familienstreit).

70 Leichte Beeinträchtigung der sozialen, beruflichen oder schulischen Leistungsfähigkeit oder leichte Symptome (z.B. leichte depressive Verstimmungen und leichte Schlaflosigkeit), jedoch im Allgemeinen recht gute Leistungsfähigkeit. Es bestehen einige wichtige zwischenmenschliche Beziehungen.

60 Mäßig ausgeprägte Störung hinsichtlich der sozialen, beruflichen oder schulischen Leistungsfähigkeit oder mäßig ausgeprägte Symptome (z.B. wenig Freunde, Konflikte mit Altersgenossen, flacher Affekt, weitschweifige Sprache, gelegentliche Panikattacken).

50 Ernsthafte Beeinträchtigung der sozialen, beruflichen oder schulischen Leistungsfähigkeit oder mäßig ausgeprägte Symptome (z.B. keine Freunde, unfähig, eine Stelle beizubehalten, Selbstmordgedanken, schwere zwanghafte Rituale, häufiger Ladendiebstahl).

40 Starke Beeinträchtigung in mehreren Bereichen, z.B. Arbeit, Schule, familiäre Beziehungen, Beurteilungsvermögen, Denken oder Stimmung (z.B. meidet ein Depressiver Freunde, vernachlässigt die Familie und ist nicht fähig zu arbeiten. Ein Kind schlägt häufig jüngere Kinder, ist zu Hause trotzig und versagt in der Schule) oder Beeinträchtigung der Realitätswahrnehmung oder der Kommunikation (z.B. die Sprache ist zeitweise unlogisch, unverständlich oder belanglos).

30 Leistungsunfähigkeit in fast allen Bereichen (bleibt z.B. den ganzen Tag im Bett, keine Arbeit, kein Zuhause oder keine Freunde) oder das Verhalten ist beträchtlich beeinflußt durch Wahnideen oder Halluzinationen oder ernsthafte Beeinträchtigung der Kommunikation bzw. des Urteilsvermögens (manchmal z.B. inkohärent, handelt weitgehend inadäquat, starke Beschäftigung mit Selbstmordgedanken).

20 Selbst- oder Fremdgefährdung (z.B. Selbstmordversuche oder eindeutige Todesabsicht, häufig gewalttätig, manische Erregung) oder ist manchmal nicht in der Lage, minimale persönliche Hygiene aufrechtzuerhalten (schmiert z.B. mit Stuhl) oder weitgehende Beeinträchtigung der Kommunikation (z.B. größtenteils inkohärent).

10 Ständige Gefahr, sich oder andere schwer zu schädigen (z.B. wiederholte Gewaltanwendung) oder anhaltende Unfähigkeit, die minimale persönliche Hygiene aufrechtzuerhalten oder ernsthafter Selbstmordversuch mit eindeutiger Todesabsicht.

Notieren Sie die entsprechende Kodierung für die Woche mit der niedrigsten Leistungsfähigkeit im vergangenen Monat. Kodieren Sie, wenn nötig, einen Zwischenwert, z.B. 68. 


\subsubsection{CGI}
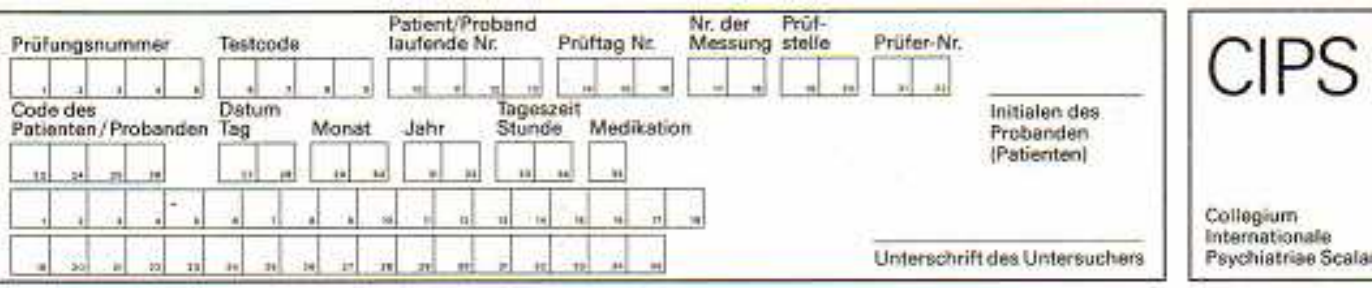

Collegium

sychiatriae Scalanom

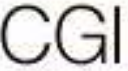

Clinicat Glob

Impressions

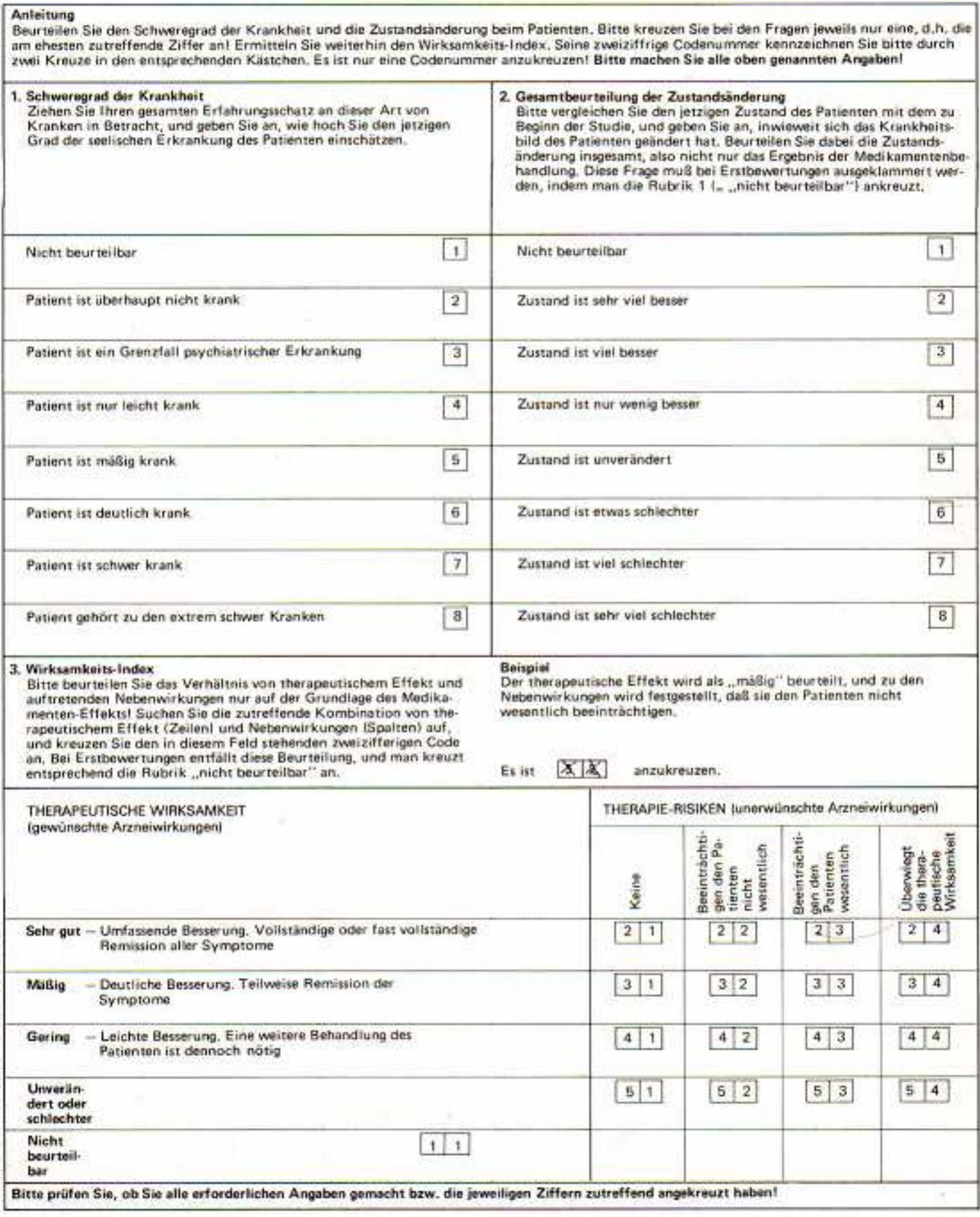




\subsubsection{BPRS}

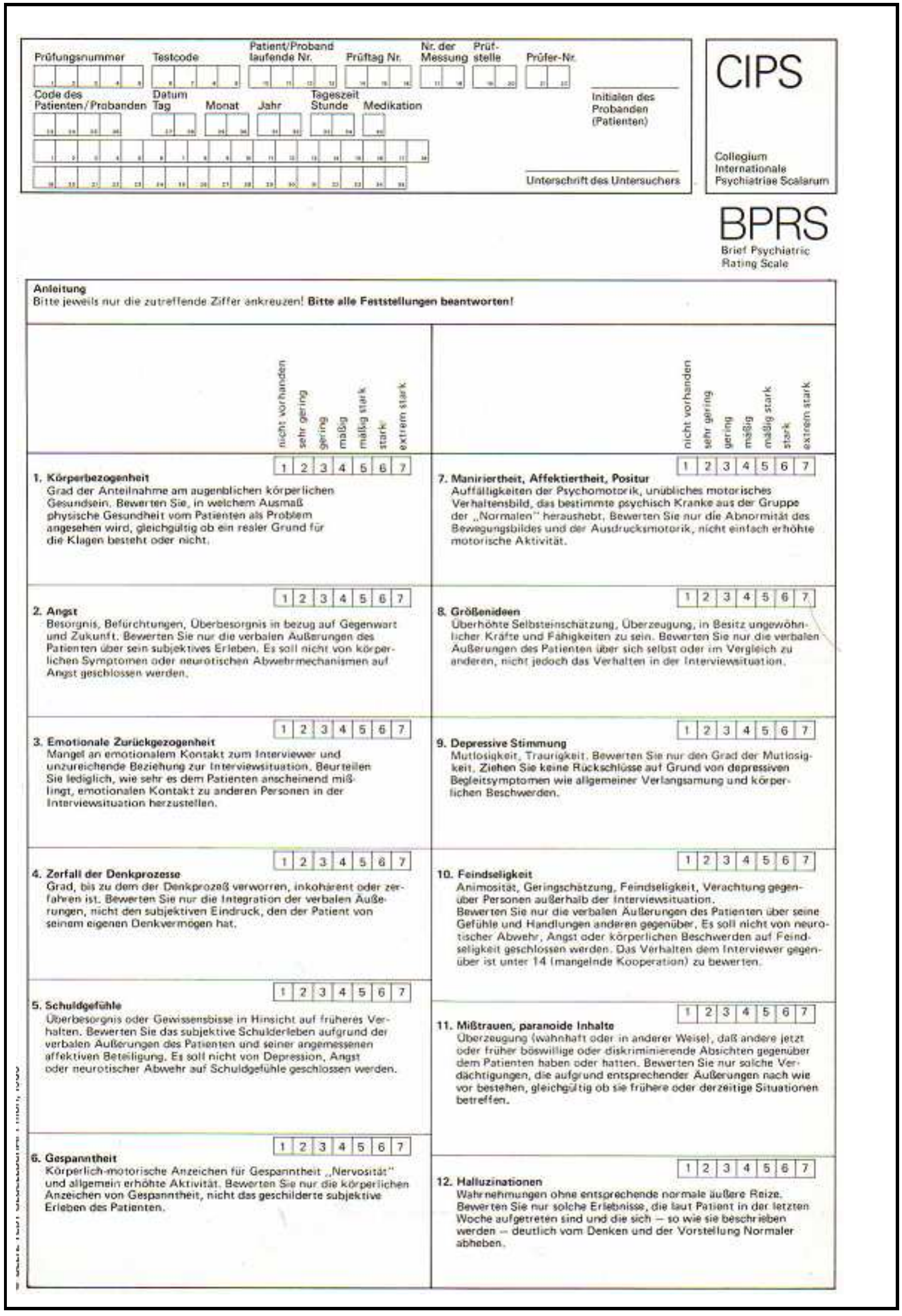




\subsubsection{BPRS (Fortsetzung)}

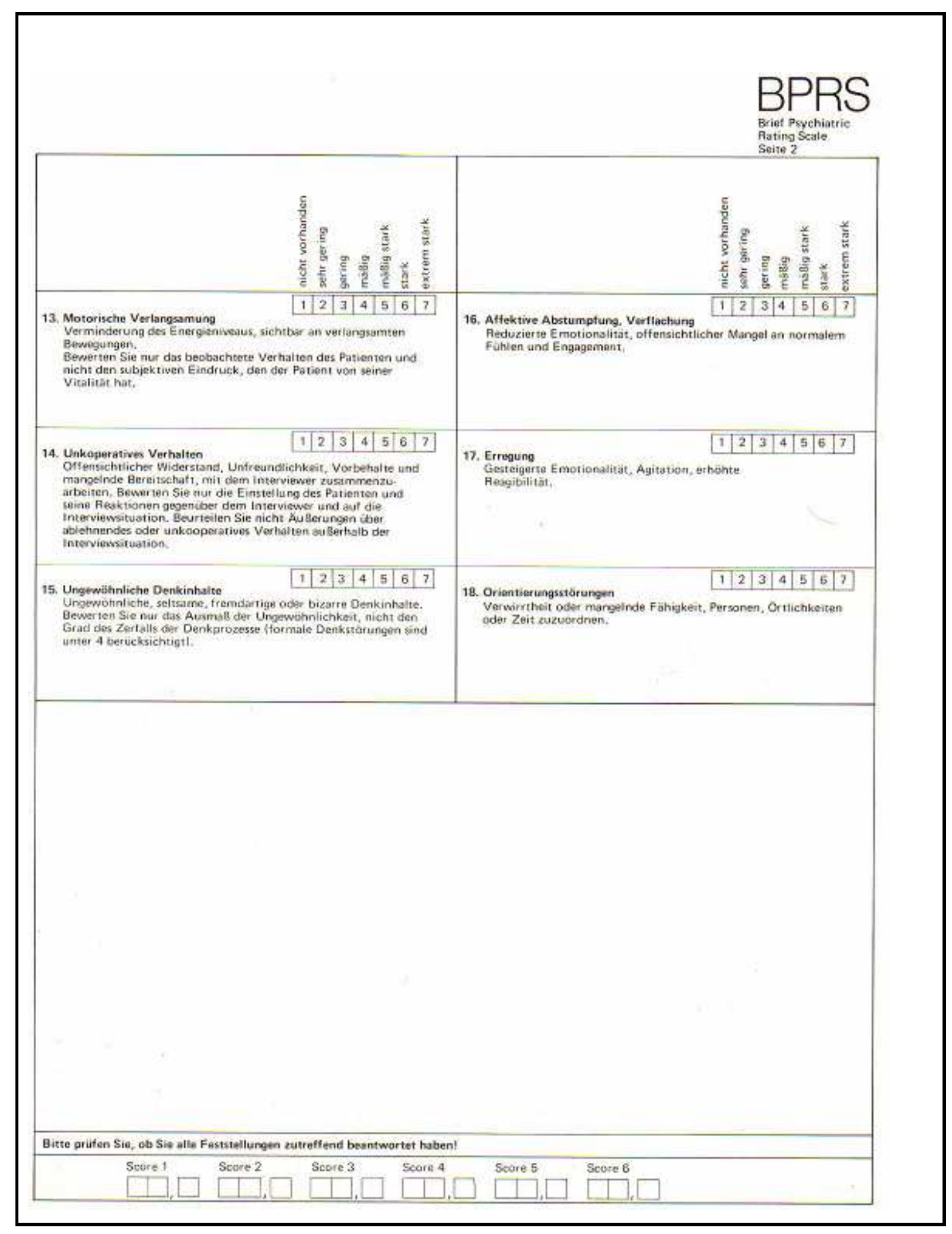




\subsubsection{SAPS}

\section{Skala für die Beurteilung positiver Symptome (SAPS)}

Beurteilungsstufen $0=$ nicht, $1=$ fraglich, $2=$ leicht, $3=$ mäßig, $4=$ ausgeprägt, $5=$ schwer

Halluzinationen

\section{Akustische Halluzinationen}

Der Patient berichtet von Stimmen, Geräuschen oder

Klängen, die niemand sonst hört.

\section{Größenideen}

Der Patient ist davon überzeugt, dass er besondere Fähigkeiten oder Macht besitzt.

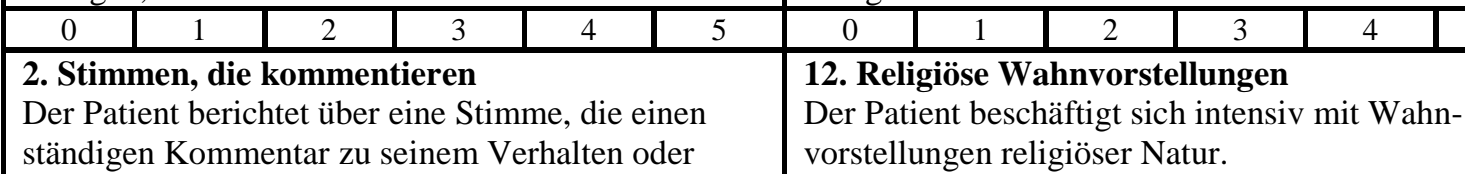
seinen Gedanken gibt.

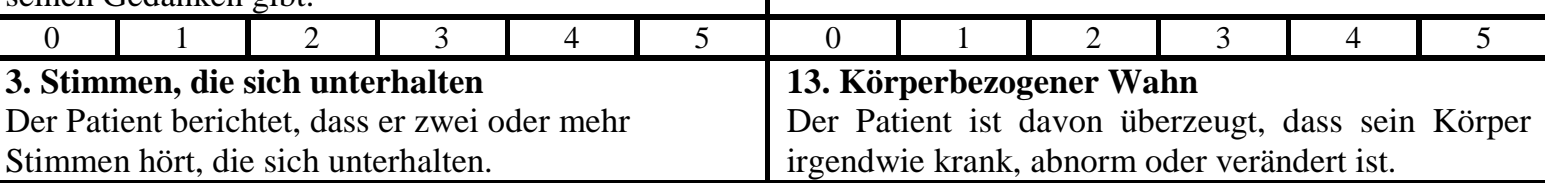

Stimmen hört, die sich unterhalten. irgendwie krank, abnorm oder verändert ist.

\begin{tabular}{|l|l|}
\hline $\begin{array}{l}\text { 4. Somatische oder taktile Halluzinationen } \\
\text { Der Patient erzählt, dass er ungewöhnliche } \\
\text { körperliche Empfindungen hat. }\end{array}$ & $\begin{array}{l}\text { 14. Beziehungsideen } \\
\text { Der Patient ist davon überzeugt, dass unbedeutende } \\
\text { Bemerkungen oder Ereignisse sich auf ihn beziehen } \\
\text { oder eine besondere Bedeutung haben. }\end{array}$ \\
\hline
\end{tabular}

\begin{tabular}{|c|c|c|c|c|c|c|c|c|c|c|}
\hline 0 & 1 & 2 & 3 & 4 & 5 & 0 & 1 & 2 & 3 & 4 \\
\hline \multicolumn{6}{|c|}{$\begin{array}{l}\text { 5. Olfaktorische Halluzinationen } \\
\text { Der Patient berichtet, dass er ungewöhnliche } \\
\text { Gerüche wahrnimmt, die niemandem sonst auffallen. }\end{array}$} & \multicolumn{5}{|c|}{$\begin{array}{l}\text { 15. Wahn, kontrolliert zu werden } \\
\text { Der Patient hat den Eindruck, dass seine Gefühle } \\
\text { oder Handlungen durch eine fremde Macht } \\
\text { kontrolliert werden. }\end{array}$} \\
\hline
\end{tabular}

\begin{tabular}{|l|c|c|c|c|c|c|c|c|c|c|}
\hline 0 & 1 & 2 & 3 & 4 & 5 & 0 & 1 & 2 & 3 & 4 \\
5
\end{tabular}

\begin{tabular}{|l|c|c|c|c|c|}
\hline 0 & 1 & 2 & 3 & 4 & 5 \\
\hline 7. Globalbeurteilung der Halluzinationen \\
Die Beurteilung sollte auf der Dauer und dem \\
Ausmaß der Halluzinationen beruhen und auf ihren \\
Auswirkungen auf das Leben des Patienten.
\end{tabular}

\begin{tabular}{|l|l|l|l|l|l|}
0 & 1 & 2 & 3 & 4 & 5 \\
\hline
\end{tabular}

\section{Gedankenausbreitung}

Der Patient ist davon überzeugt, dass seine Gedanken ausgestrahlt werden, so dass sie von ihm oder anderen gehört werden können.

\begin{tabular}{|l|l|l|l|l|l|}
\hline 0 & 1 & 2 & 3 & 4 & 5 \\
\hline Wahnphänomene \\
\hline 8. Verfolgungswahn \\
Der Patient ist davon überzeugt, dass eine Verschwö- \\
rung gegen ihn besteht oder dass er von jemandem \\
verfolgt wird.
\end{tabular}

\begin{tabular}{|l|l|l|l|l|l}
0 & 1 & 2 & 3 & 4 & 5 \\
\hline
\end{tabular}

\section{Gedankeneingebung}

Der Patient ist davon überzeugt, dass ihm fremde

Gedanken eingegeben werden. verfolgt wird

\begin{tabular}{|l|c|c|c|c|c|c|c|c|c|c|}
\hline 0 & 1 & 2 & 3 & 4 & 5 & 0 & 1 & 2 & 3 & 4 \\
5 \\
\hline $\begin{array}{l}\text { 9. Eifersuchtswahn } \\
\text { Der Patient ist davon überzeugt, dass sein Partner } \\
\text { eine Affäre mit einem anderen hat. }\end{array}$
\end{tabular}




\subsubsection{SANS}

\section{Skala für die Beurteilung negativer Symptome (SANS)}

Beurteilungsstufen $0=$ nicht, $1=$ fraglich, $2=$ leicht, $3=$ mäßig, $4=$ ausgeprägt, $5=$ schwer Affektive Verflachung oder Abstumpfung

\section{Starrer Gesichtsausdruck}

Das Gesicht des Patienten erscheint hölzern, es ändert sich weniger als erwartet, wenn der

Gesprächsinhalt wechselt.

\section{Vergrößerte Antwortlatenz}

Der Patient benötigt viel Zeit, um Fragen zu beantworten. Eine Nachfrage zeigt, dass der Patient die Frage verstanden hat.

\begin{tabular}{|l|l|l|l|l|l|}
0 & 1 & 2 & 3 & 4 & 5 \\
\hline
\end{tabular}

2. Verminderte Spontanbewegungen

Der Patient zeigt wenig oder gar keine Spontanbewegungen, er wechselt seine Sitzposition nicht, bewegt die Extremitäten nicht.

\begin{tabular}{|l|l|l|l|l|l|}
\hline 0 & 1 & 2 & 3 & 4 & 5 \\
\hline
\end{tabular}

\section{Fehlen expressiver Gesten}

Der Patient setzt weder Handbewegungen, noch den Körper als Hilfe für den Ausdruck seiner Vorstellungen ein.

\begin{tabular}{|c|c|c|c|c|c|}
\hline 0 & 1 & 2 & 3 & 4 & 5 \\
\hline \multicolumn{6}{|c|}{$\begin{array}{l}\text { 4. Unzureichender Blickkontakt } \\
\text { Der Patient meidet den Blickkontakt oder „starrt“ } \\
\text { durch den Interviewer hindurch, sogar wenn er } \\
\text { spricht. }\end{array}$} \\
\hline 0 & 1 & 2 & 3 & 4 & 5 \\
\hline
\end{tabular}

\section{Fehlende affektive Reaktionen}

Der Patient lächelt oder lacht nicht, wenn diese

Reaktion zu erwarten ist.

\section{Globalbeurteilung der Alogie}

Die Hauptmerkmale der Alogie sind Reduzierung des Sprachumfangs und des Sprachinhalts.

\begin{tabular}{|l|l|l|l|l|l|}
\hline 0 & 1 & 2 & 3 & 4 & 5 \\
\hline
\end{tabular}

\section{Willensschwäche - Apathie}

\section{Kleidung und Hygiene}

Die Kleidung des Patienten kann nachlässig oder verschmutzt sein und er kann fettige Haare,

Körpergeruch usw. haben.

\section{Unbeständigkeit bei der Arbeit oder in der} Schule zu finden oder aufrechtzuerhalten, seine Hausaufgaben zu machen, den Haushalt zu versorgen usw. ist dieser nicht in der Lage, an den Aktivitäten auf der Station teilzunehmen (Beschäftigungstherapie,
Der Patient hat Schwierigkeiten, eine Beschäftigung Wenn es sich um einen stationären Patienten handelt, Kartenspiele usw.)

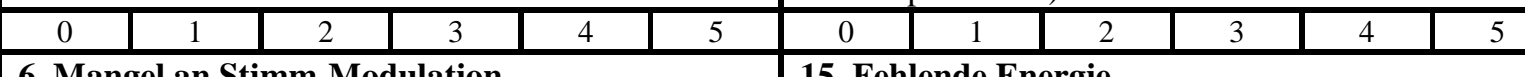

6. Mangel an Stimm-Modulation

Der Patient zeigt zur Betonung des Sprachinhalts keine normale Modulation, er spricht monoton.

15. Fehlende Energie

Der Patient neigt zur Untätigkeit. Er kann

stundenlang ruhig sitzen und initiiert keinerlei spontane Aktivität.

\begin{tabular}{|l|l|l|l|l|l|l|l|l|l|l|}
\hline 0 & 1 & 2 & 3 & 4 & 5 & 0 & 1 & 2 & 3 & 4 \\
5
\end{tabular}

\begin{tabular}{|l|l|l|l|l|l|}
\hline 0 & 1 & 2 & 3 & 4 & 5 \\
\hline Alogie \\
\hline 8. Reduzierung des Sprachumfangs
\end{tabular}

\section{Reduzierung des Sprachumfangs}

Die Antworten des Patienten auf Fragen sind im Umfang begrenzt, eher kurz, konkret und wenig elaboriert.

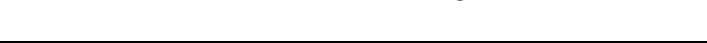

\begin{tabular}{c|c|c|c|c|c|}
\hline & 1 & 2 & 3 & 4 & 5 \\
\hline
\end{tabular}

\section{Interesse an Freizeitaktivitäten}

Der Patient kann sehr wenig oder kein Interesse haben. Sowohl die Zahl, als auch die Qualität des Interesses sollte berücksichtigt werden.

\begin{tabular}{|c|c|c|c|c|c|c|c|c|c|c|c|}
\hline 0 & 1 & 2 & 3 & 4 & 5 & 0 & 1 & 2 & 3 & 4 & 5 \\
\hline \multicolumn{6}{|c|}{$\begin{array}{l}\text { 9. Reduzierung des Sprachinhalts } \\
\text { Die Antworten des Patienten sind vom Umfang her } \\
\text { angemessen, bleiben aber vage, zu sehr detail- } \\
\text { gebunden oder zu allgemein und übermitteln wenig }\end{array}$} & \multicolumn{6}{|c|}{$\begin{array}{l}\text { 18. Sexuelle Aktivität } \\
\text { Der Patient zeigt vermindertes sexuelles Interesse } \\
\text { und Aktivität. Wenn er jedoch aktiv bleibt, ist die } \\
\text { Freude daran beeinträchtigt. }\end{array}$} \\
\hline
\end{tabular}

\begin{tabular}{|c|c|c|c|c|c|c|c|c|c|c|c|}
\hline 0 & 1 & 2 & 3 & 4 & 5 & 0 & 1 & 2 & 3 & 4 & 5 \\
\hline \multicolumn{6}{|c|}{$\begin{array}{l}\text { 10. Gedankensperre } \\
\text { Der Patient zeigt entweder spontan oder auf Nach- } \\
\text { frage, dass sein Gedankengang unterbrochen wurde. }\end{array}$} & \multicolumn{6}{|c|}{$\begin{array}{l}\text { 19. Die Fähigkeit, Intimität und Nähe zu erleben } \\
\text { Der Patient kann eine Unfähigkeit zeigen, enge oder } \\
\text { intime Beziehungen aufzubauen, besonders zum } \\
\text { anderen Geschlecht und der Familie. }\end{array}$} \\
\hline 0 & 1 & 2 & 3 & 4 & 5 & 0 & 1 & 2 & 3 & 4 & 5 \\
\hline
\end{tabular}




\subsubsection{SANS (Fortsetzung)}

\begin{tabular}{|ll}
\hline Skala für die Beurteilung negativer Symptome (SANS) (Fortsetzung) \\
\hline
\end{tabular}

Beurteilungsstufen $0=$ nicht, $1=$ fraglich, $2=$ leicht, $3=$ mäßig, $4=$ ausgeprägt, $5=$ schwer

\begin{tabular}{|l|l|}
\hline $\begin{array}{l}\text { 20. Beziehungen zu Freunden und Alleinsein } \\
\text { oollen }\end{array}$ & $\begin{array}{l}\text { 23. Unaufmerksamkeit während der Prüfung des } \\
\text { pyifchen Status }\end{array}$
\end{tabular}
wollen

Der Patient hat wenige oder keine Freunde und zieht psychischen Status

es vor, seine ganze Zeit allein zu verbringen.

Serielle Subtraktion von „7“ (mindestens fünf

Subtraktionen) und rückwärts buchstabieren des

\begin{tabular}{|l|l|l|l|l|l|}
\hline 0 & 1 & 2 & 3 & 4 & 5 \\
\hline
\end{tabular}

Wortes „Radio“. Beurteilung: 2 = 1 Fehler; $3=2$

21. Globalbeurteilung der Anhedonie -

Fehler; 4 = 3 Fehler. Ungeselligkeit

Diese Beurteilung sollte den Schweregrad widerspiegeln wobei das Alter des Patienten, sein

Familienstatus usw. zu berücksichtigen sind.

24. Globalbeurteilung der Aufmerksamkeit

Die Beurteilung sollte sich auf das Konzentrations-

vermögen sowohl beim Interview, als auch bei den

Tests beziehen.

\begin{tabular}{|c|c|c|c|c|c|}
\hline 0 & 1 & 2 & 3 & 4 & 5 \\
\hline \multicolumn{6}{|c|}{ Aufmerksamkeit } \\
\hline \multicolumn{6}{|c|}{$\begin{array}{l}\text { 22. Fehlende Aufmerksamkeit in sozialen } \\
\text { Situationen } \\
\text { Der Patient macht einen unbeteiligten Eindruck. Er } \\
\text { ist ,weit weg“. }\end{array}$} \\
\hline 0 & 1 & 2 & 3 & 4 & 5 \\
\hline
\end{tabular}




\subsubsection{Virtuelle Realität}

\subsubsection{Aufklärungstext VR}

\section{Erläuterung der Untersuchung «VR-gestütztes Testsystem zur Diagnose neuropsychologischer Erkrankungen»}

Sehr geehrte Dame, sehr geehrter Herr,

Sie nehmen an einer wissenschaftlichen Untersuchung teil, die in der Abteilung für Psychopathologie und Neuropsychologie des Uniklinikums Göttingen durchgeführt wird. Das Ziel dieser Untersuchung ist die Entwicklung und Erprobung eines neuen Testsystems zur Diagnose von Konzentrations- und Gedächtnisstörungen.

Das Testsystem präsentiert dem Betrachter per Computer eine fremde räumliche Umgebung (z.B. eine Landschaft), die gelernt werden soll. Die räumliche Umgebung wird als eine sogenannte „Virtuelle Realität“ dargeboten. Der Betrachter trägt eine Datenbrille, auf der die Computergraphik erscheint.

In seltenen Fällen kann es dabei zum Auftreten von Übelkeit, Kopfschmerzen oder Doppelbildern kommen. In diesem Fall sollten Sie den Gedächtnistest sofort abbrechen. Auch wenn Sie sich erschöpft, unwohl oder unsicher fühlen, sollten Sie den Versuch sofort beenden und dem Versuchsleiter ihre Probleme oder Schwierigkeiten mitteilen. Sie können unabhängig von den vorgesehenen Pausen jederzeit weitere Pausen einlegen, falls Sie es wünschen.

Sie haben weiterhin jederzeit das Recht, ohne Angabe von Gründen die Untersuchung zu beenden. Ihnen entstehen daraus keine Nachteile hinsichtlich Ihrer Betreuung und Behandlung.

Alle von Ihnen erhobenen Daten werden anonym ausgewertet und gemäß der geltenden rechtlichen Bestimmungen (Paragraph 203 StGB) vor unbefugter Offenbarung strikt geschützt.

Prof. Dr. O. Rienhoff

(Abt. medizinische Informatik)

Dr. G. Weniger

(Abt. Psychiatrie,

Schwerpunkt Neuropsychologie)
Prof. Dr. E. Irle

(Abt. Psychiatrie,

Schwerpunkt Neuropsychologie) 


\subsubsection{Instruktionen (via HMD präsentiert)}

„Vielen Dank für Ihre Teilnahme an dieser Untersuchung. Es soll getestet werden, wie Sie sich in einer fremden Umgebung orientieren können. Diese fremde Umgebung wird Ihnen vom Computer präsentiert. Sie werden dabei eine Datenbrille tragen, auf der Computergraphiken erscheinen.

Bevor Sie nun mit der Lernaufgabe beginnen, üben Sie bitte den Umgang mit den technischen Geräten. Um sich in der Umgebung fortzubewegen haben Sie folgende Möglichkeiten:

个 - Taste: Laufen des Weges bis zur nächsten Abzweigung

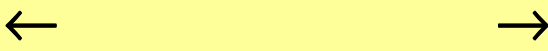

- Taste:

- Taste:

nach links drehen

nach rechts drehen

- Taste: Umdrehen und zurücklaufen des Weges bis zur letzten Abzweigung

Üben Sie den Umgang mit den technischen Geräten bitte so lange, bis Sie sich sicher fühlen. Wenn Sie das Gefühl haben, dass Sie sich sicher durch das Labyrinth/den Park bewegen können oder wenn die Übungsphase vorbei ist, beendet der Versuchsleiter die Übungsphase.

Haben Sie noch Fragen?"

Abbildung 12: Instruktion Trainingstrials 


\subsubsection{Instruktionen (via HMD präsentiert); (Fortsetzung)}

„Sie werden gleich einen Park sehen, der von einem Wegenetz durchzogen ist. Viele Straßen enden darin in Sackgassen. An den Enden der Sackgassen befinden sich große Töpfe. Fast alle Töpfe sind leer, nur in einem Topf sind Münzen versteckt. Man sieht diese Münzen noch nicht aus der Ferne, sondern erst, wenn man direkt vor dem Topf steht.

$\rightarrow$ Ihre Aufgabe ist es, denjenigen Topf zu entdecken, in dem die Münzen versteckt sind.

$\rightarrow$ Gehen Sie dabei bitte möglichst geschickt/sparsam vor, d.h. versuchen Sie so selten wie möglich in demselben Topf zweimal nachzuschauen.

$\rightarrow$ Merken Sie sich, wo der gefüllte Topf steht, da Sie ihn später wiederfinden sollen.

Haben Sie noch Fragen?“

Abbildung 13: Instruktion für 1. Testdurchgang Park

„Sie haben gerade entdeckt, in welchem Topf sich die Münzen befinden. Im kommenden Durchgang starten Sie erneut am Ausgangspunkt. Da Ihnen nun bekannt ist, welcher Topf gefüllt ist, gehen Sie bitte auf direktem Wege dorthin.

$\rightarrow$ Laufen Sie bitte direkt zu demjenigen Topf, in dem die Münzen versteckt sind.

$\rightarrow$ Gehen Sie dabei bitte möglichst geschickt/sparsam vor, d.h. vermeiden Sie unnötige Umwege.

Haben Sie noch Fragen?“ 


\subsubsection{Nachbefragungsbogen VR}

\section{VR-Projekt: NACHBEFRAGUNG \\ VpNr.: \\ Datum:}

1. Ich empfand die Aufgabe insgesamt...

im Park: $\quad$ sehr schwer 01 --- 02 --- 03 --- 04 --- 05 sehr leicht

im Labyrinth: $\quad$ sehr schwer 01 --- 02 --- 03 --- 04 --- 05 sehr leicht

2. Um den Park beim Kennenlernen sinnvoll durchsuchen zu können, habe ich folgende Strategie benutzt:

O gar keine

O ich habe immer möglichst sofort eine Sackgasse durchsucht, wenn ich an eine Gabelung kam

O ich habe das Betreten von Sackgassen vermieden

o andere Strategie, und zwar:

3. Um das Ziel im zweiten (dritten usw.) Durchgang schnell wiederzufinden, habe ich mir im Park Folgendes eingeprägt:

O eine Abfolge von Richtungswechseln (z.B. rechts, links, links, ...)

O eine Abfolge von markanten Orten und Richtungswechseln (z.B. am Spielplatz links, vor der Brücke rechts, am Backsteinhaus rechts, ...)

O einen räumlichen Überblick über die Landschaft und über die Form des Weges

O etwas anderes:

4. Um das Labyrinth beim Kennenlernen sinnvoll durchsuchen zu können, habe ich folgende Strategie benutzt:

O gar keine

O ich habe immer möglichst sofort eine Sackgasse durchsucht, wenn ich an eine Gabelung kam

O ich habe das Betreten von Sackgassen vermieden

O andere Strategie, und zwar:

5. Um das Ziel im zweiten (dritten usw.) Durchgang schnell wiederzufinden, habe ich mir im Labyrinth Folgendes eingeprägt:

O eine Abfolge von Richtungswechseln (z.B. rechts, links, links, ...)

O einen räumlichen Überblick über die Landschaft und über die Form des Weges

O etwas anderes: 


\subsubsection{Nachbefragungsbogen VR (Fortsetzung)}

6. Bei der Fortbewegung bin ich mit Datenhelm und Tastatur zurechtgekommen.

O ja O nein, ich hatte Schwierigkeiten mit

7. Die Benutzung des Datenhelms war für mich unangenehm/beängstigend.

O ja O nein

8. Die Erklärung des Testablaufs, die Anweisungen und die Fragen waren verständlich. O ja $\quad O$ nein, mir war Folgendes unklar:

9. Die virtuellen Umgebungen erschienen mir realistisch.

Park: $\quad$ wenig realistisch 01 --- 02 --- 03 --- 04 --- 05 sehr realistisch

Labyrinth: $\quad$ wenig realistisch 01 --- 02 --- 03 --- 04 --- 05 sehr realistisch

10. Ich habe mich in die Umgebungen hineinversetzt gefühlt.

Park: $\quad$ kaum 01 --- 02 --- 03 --- 04 --- 05 sehr stark

Labyrinth: $\quad$ kaum 01 --- 02 --- 03 --- 04 --- 05 sehr stark

11. Ich habe während des Experiments Eindrücke außerhalb der virtuellen Umgebungen wahrgenommen.

$$
\text { kaum } 01 \text {--- } 02 \text {--- } 03 \text {--- } 04 \text {--- } 05 \text { sehr stark }
$$

12. Insgesamt war die Erfahrung mit dem Testsystem ... (z.B. langweilig, interessant, frustrierend):

Park:

Labyrinth:

13. Ich hatte schon Vorerfahrungen mit dem Computer:

O gar keine

O vereinzelte Erfahrungen / Grundkenntnisse (z.B. in Textverarbeitung)

O häufige Erfahrungen / gute Kenntnisse (z.B. Grafikverarb., Computerspiele, Programmieren)

14. Im Alltag ist mein Orientierungsvermögen... sehr schlecht 01 --- 02 --- 03 --- 04 --- 05 sehr gut 
7.2.4.4. Rekognitionsbogen (Version: Labyrinth)

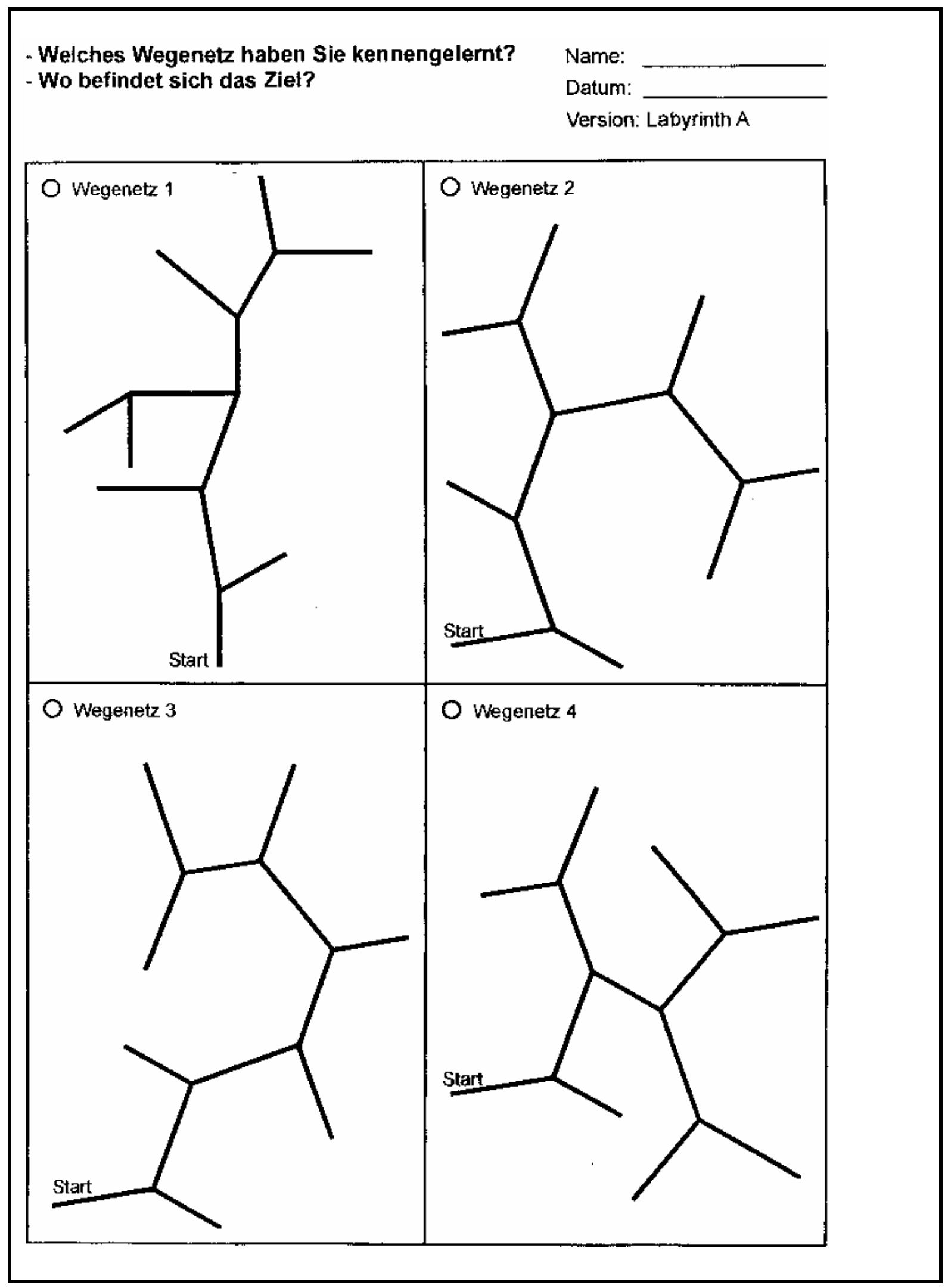




\subsubsection{Magnetresonanztomographie: Aufklärungstext}

\section{Kernspin-Untersuchung (Magnetresonanztomographie-Untersuchung)}

Die magnetresonanztomographische Untersuchung dient dazu, Aufschlüsse über die morphologische Struktur und Beschaffenheit des Gehirns zu bekommen. Solche Untersuchungen werden im Bereich der Neurologie und Psychiatrie routinemäßig bei sehr vielen Patienten eingesetzt um etwaige Hirnerkrankungen auszuschließen.

Die Untersuchung ist absolut ungefährlich. Es wird nicht mit Strahlen gearbeitet, sondern mit einem starken Magnetfeld. Aufgrund des starken Magnetfelds darf diese Untersuchung jedoch nicht bei Personen durchgeführt werden, bei denen sich Metallteile (z.B. Herzschrittmacher, Metallplatten, Klammern) im Körper befinden.

Die Untersuchung dauert ca. 15 Minuten. Sie werden in einer relativ engen Röhre, aber trotzdem bequem, gelagert werden. Sie sollen in dieser Zeit einfach ruhig daliegen und sich entspannen. Durch das Magnetfeld entstehen starke Geräuscheinwirkungen, die jedoch durch Ohrstöpsel gedämpft werden. Sie haben jederzeit die Möglichkeit, über Mikrophon mit dem Untersuchungspersonal zu sprechen oder einen Signalkopf zu drücken.

\section{Nebenwirkungen und Risiken der Untersuchung}

Für psychiatrische und neuropsychologische Untersuchungen sind bei fachgerechter Durchführung keine Risiken bekannt.

Die Untersuchung im Magnetresonanztomographen kann durch eine längere Liegedauer, und durch die Tatsache, dass Sie Ihren Kopf während der Untersuchung nicht bewegen dürfen, als unbequem empfunden werden. Wir werden uns aber darum bemühen, Sie so bequem wie möglich zu lagern. Es ist sehr wichtig, dass Sie während der Untersuchung ruhig liegen und vor allem den Kopf nicht bewegen. 


\subsection{Technische Informationen}

\subsubsection{VR: Technische Daten/Aufbau (PC)}

\section{Hardware Configuration}

- $\quad$ Pentium-based PC $233 \mathrm{MHz}$

- $128 \mathrm{MB}$ RAM

- 2 Voodoo2-Add-on graphic cards

- 4 MB Texture Memory

- OpenGL-Acceleration

\section{Software Configuration}

- Windows NT

- Visualisation tool

- $\quad$ Kinetix 3D Studio Max R2

- Programming:

- Realimation VSG 4.4

- $\quad$ Microsoft $\mathrm{C}++$

- Division dv/reality

\section{$\underline{\text { 7.3.2. Magnetresonanztomographie: Scanparameter und Aufnahmeinstellungen }}$}

\begin{tabular}{|lc|ll|}
\hline & \multicolumn{1}{l|}{ T1-gewichtet } & Aufnahmemodus: & 3-D \\
Sequenz: & $256 \times 256$ Pixel & Schichtebene: & sagittal \\
Matrix: & $1,0 \mathrm{~mm}$ & Schichtanzahl: & 256 \\
Schichtdicke: & $6.0 \mathrm{msec}$ & Wiederholungszeit (TR): & $24.0 \mathrm{msec}$ \\
Echozeit (TE): & 2 & Aufnahmewinkel: & $30^{\circ}$ \\
Anzahl der Anregungen: & & \\
Sichtfeld/field of view: & 256 & & \\
\hline
\end{tabular}




\subsection{Ergänzende statistische Kennwerte}

\subsubsection{Ergänzung zu 4.1.3.1. (Zusammenhänge Neuropsychologie - Hirnvolumetrie)}

Tabelle 26: Zusammenhang zwischen der Navigationsleistung im egozentrischen Raum \& parietalen Hirnvolumina (Kontrollgruppe, $n=25$ )

\begin{tabular}{|c|c|c|c|c|c|c|}
\hline $\begin{array}{c}\text { Navigations- } \\
\text { leistung: } \\
\text { Cortexareal: }\end{array}$ & $\begin{array}{l}\text { Fehler- } \\
\text { summe I }\end{array}$ & $\begin{array}{c}\text { Fehler- } \\
\text { summe II }\end{array}$ & $\underline{\text { Zeit } I^{2}}$ & $\underline{\text { Zeit II }}$ & $\underbrace{\text { Lern- }}_{\text {gewinn } I^{2}}$ & $\stackrel{\frac{\text { Lern- }}{\text { gewinn II }}}{\text { gewing }}$ \\
\hline & $\underline{\mathbf{r}}_{\mathrm{s}}:$ & $\underline{\mathbf{r}}_{\mathrm{s}}:$ & $\underline{\mathbf{r}}:$ & $\underline{\mathbf{r}}:$ & $\underline{\mathbf{r}}:$ & $\underline{\mathbf{r}}:$ \\
\hline $\begin{array}{c}\text { Parietallappen } \\
\text { rechts }\end{array}$ & .050 & .190 & .052 & .203 & -.154 & .210 \\
\hline $\begin{array}{l}\text { Parietallappen } \\
\text { links }\end{array}$ & .254 & .105 & .267 & .067 & -.130 & -.246 \\
\hline $\begin{array}{c}\text { Superiorer PL } \\
\text { rechts }\end{array}$ & .001 & .081 & .112 & .149 & -.105 & .084 \\
\hline $\begin{array}{l}\text { Superiorer PL } \\
\text { links }\end{array}$ & .034 & -.059 & .116 & -.048 & -.015 & -.190 \\
\hline $\begin{array}{c}\text { Inferiorer PL } \\
\text { rechts }\end{array}$ & -.082 & .244 & -.077 & .211 & -.173 & .344 \\
\hline $\begin{array}{c}\text { Inferiorer PL } \\
\text { links }\end{array}$ & .176 & .177 & .396 & .232 & -.265 & -.219 \\
\hline
\end{tabular}

Testverfahren und Prüfgröße: ${ }^{1}$ Spearman Rangkorrelation, $\mathrm{r}_{\mathrm{s}} ;{ }^{2}$ Pearson-Korrelation, $\mathrm{r}$.

Tabelle 27: $\quad$ Zusammenhang zwischen der Navigationsleistung im allozentrischen Raum \& parietalen Hirnvolumina (Kontrollgruppe, $n=25$ )

\begin{tabular}{|c|c|c|c|c|c|c|}
\hline $\begin{array}{c}\text { Navigations- } \\
\text { leistung: } \\
\text { Cortexareal: }\end{array}$ & $\begin{array}{l}\text { Fehler- } \\
\text { summe I }\end{array}$ & $\begin{array}{c}\text { Fehler- } \\
\text { summe II }^{1}\end{array}$ & $\underline{\text { Zeit } \mathbf{I}^{2}}$ & $\underline{\text { Zeit II }^{2}}$ & $\underline{\text { Lewn- }}_{\text {gewinn } I^{2}}$ & $\underline{\underline{\text { Lern- }}}{\underline{\text { gewinn } \text { II }^{2}}}^{2}$ \\
\hline & $\underline{\mathbf{r}}_{\mathrm{s}}:$ & $\underline{\mathbf{r}}_{\mathrm{s}}:$ & $\underline{\mathbf{r}}:$ & $\underline{\mathbf{r}}:$ & $\underline{\mathbf{r}}:$ & $\underline{\mathbf{r}}:$ \\
\hline $\begin{array}{l}\text { Parietallappen } \\
\text { rechts }\end{array}$ & .207 & -.014 & .081 & -.076 & -.206 & -.011 \\
\hline $\begin{array}{c}\text { Parietallappen } \\
\text { links }\end{array}$ & .275 & -.005 & .064 & -.124 & -.235 & -.074 \\
\hline $\begin{array}{c}\text { Superiorer PL } \\
\text { rechts }\end{array}$ & .035 & -.159 & -.009 & -.217 & -.141 & -.110 \\
\hline $\begin{array}{c}\text { Superiorer PL } \\
\text { links }\end{array}$ & .093 & -.110 & -.034 & -.209 & -.175 & -.027 \\
\hline $\begin{array}{l}\text { Inferiorer PL } \\
\text { rechts }\end{array}$ & .267 & .114 & .206 & .207 & -.234 & .168 \\
\hline $\begin{array}{c}\text { Inferiorer PL } \\
\text { links }\end{array}$ & .145 & -.095 & .202 & .086 & -.221 & -.119 \\
\hline
\end{tabular}

Testverfahren und Prüfgröße: ${ }^{1}$ Spearman Rangkorrelation, $r_{s} ;{ }^{2}$ Pearson-Korrelation, $r$. 


\subsubsection{Ergänzung zu 4.2.1. (Hippocampale Areale)}

Tabelle 28: Zusammenhang zwischen der Navigationsleistung im egozentrischen Raum \& hippocampalen Hirnvolumina; (Kontrollgruppe, $n=25$ )

\begin{tabular}{|c|c|c|c|c|c|c|}
\hline $\begin{array}{l}\text { Navigations- } \\
\text { leistung: } \\
\text { Cortexareal: }\end{array}$ & $\begin{array}{l}\text { Fehler- } \\
\text { summe I }\end{array}$ & $\begin{array}{c}\text { Fehler- } \\
\text { summe II }\end{array}$ & $\underline{\text { Zeit } I^{2}}$ & $\underline{\text { Zeit II }^{2}}$ & $\frac{\text { Lern- }}{\text { gewinn } \mathbf{I}^{2}}$ & 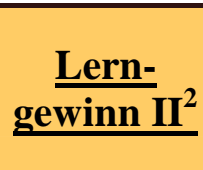 \\
\hline & $\underline{\mathbf{r}}_{\mathrm{s}}:$ & $\underline{\mathbf{r}}_{\mathrm{s}}:$ & $\underline{\mathbf{r}}:$ & $\underline{\mathbf{r}}:$ & $\underline{\mathbf{r}}:$ & $\underline{\mathbf{r}}:$ \\
\hline $\begin{array}{l}\text { Hippocampus } \\
\text { rechts }\end{array}$ & -.013 & .224 & .043 & .259 & -.147 & .151 \\
\hline $\begin{array}{c}\text { Hippocampus } \\
\text { links }\end{array}$ & .112 & 279 & .198 & .331 & -.154 & .106 \\
\hline
\end{tabular}

Testverfahren und Prüfgröße: ${ }^{1}$ Spearman Rangkorrelation, $r_{s} ;{ }^{2}$ Pearson-Korrelation, $r$.

Tabelle 29: Zusammenhang zwischen der Navigationsleistung im allozentrischen Raum \& hippocampalen Hirnvolumina; (Kontrollgruppe, $n=25$ )

\begin{tabular}{|c|c|c|c|c|c|c|}
\hline $\begin{array}{l}\frac{\text { Navigations- }}{\text { leistung: }} \\
\text { Cortexareal: }\end{array}$ & $\begin{array}{c}\text { Fehler- } \\
\text { summe I } \\
\underline{\underline{n}}\end{array}$ & $\begin{array}{c}\underline{\text { Fehler- }}^{\text {summe II }} \\
\underline{\underline{1}}\end{array}$ & $\underline{\text { Zeit I }^{2}}$ & $\underline{\text { Zeit II }^{2}}$ & $\frac{\text { Lern- }}{\text { gewinn } I^{2}}$ & $\begin{array}{l}\frac{\text { Lern- }}{\text { gewinn II }} \\
\underline{\text { w }}\end{array}$ \\
\hline & $\underline{\mathbf{r}}_{\mathrm{s}}:$ & $\underline{\mathbf{r}}_{\mathrm{s}}:$ & $\underline{\mathbf{r}}:$ & $\underline{\mathbf{r}}:$ & $\underline{\mathbf{r}}:$ & $\underline{\mathbf{r}}:$ \\
\hline $\begin{array}{l}\text { Hippocampus } \\
\text { rechts }\end{array}$ & .143 & -.056 & -.004 & -.101 & -.121 & -.201 \\
\hline $\begin{array}{c}\text { Hippocampus } \\
\text { links }\end{array}$ & .051 & -.141 & -.035 & -.217 & -.057 & -.335 \\
\hline
\end{tabular}

Testverfahren und Prüfgröße: ${ }^{1}$ Spearman Rangkorrelation, $r_{s} ;{ }^{2}$ Pearson-Korrelation, $r$.

Tabelle 30: Zusammenhang zwischen der Navigationsleistung im egozentrischen Raum \& hippocampalen Hirnvolumina; (Patientengruppe, $n=21$ )

\begin{tabular}{|c|c|c|c|c|c|c|}
\hline $\begin{array}{c}\text { Navigations- } \\
\text { leistung: } \\
\text { Cortexareal: }\end{array}$ & $\begin{array}{l}\text { Fehler- } \\
\text { summe I }\end{array}$ & $\begin{array}{c}\text { Fehler- } \\
\text { summe II }^{1}\end{array}$ & $\underline{\text { Zeit I }^{2}}$ & $\underline{\text { Zeit II }^{2}}$ & $\underset{\text { gewinn } I^{2}}{\underline{\text { Lern- }}}$ & $\frac{\text { Lern- }}{\text { gewinn II }}$ \\
\hline & $\underline{\mathbf{r}}_{\mathrm{s}}:$ & $\underline{\mathbf{r}}_{\mathrm{s}}:$ & $\underline{\mathbf{r}}:$ & $\underline{\mathbf{r}}:$ & $\underline{\mathbf{r}}:$ & $\underline{\mathbf{r}}:$ \\
\hline $\begin{array}{c}\text { Hippocampus } \\
\text { rechts }\end{array}$ & .080 & .032 & .035 & .174 & -.037 & .119 \\
\hline $\begin{array}{l}\text { Hippocampus } \\
\text { links }\end{array}$ & .371 & .148 & .353 & .222 & -.335 & -.362 \\
\hline
\end{tabular}

Testverfahren und Prüfgröße: ${ }^{1}$ Spearman Rangkorrelation, $r_{s} ;{ }^{2}$ Pearson-Korrelation, $r$. 
Tabelle 31: Zusammenhang zwischen der Navigationsleistung im allozentrischen Raum \& hippocampalen Hirnvolumina; (Patientengruppe, $n=21$ )

\begin{tabular}{|c|c|c|c|c|c|c|}
\hline $\begin{array}{c}\text { Navigations- } \\
\text { leistung: } \\
\text { Cortexareal: }\end{array}$ & $\begin{array}{l}\text { Fehler- } \\
\text { summe I } \\
\underline{\text { unmment }}\end{array}$ & $\begin{array}{c}\underline{\text { Fehler- }}^{\text {summe II }} \\
\underline{u}^{1}\end{array}$ & $\underline{\text { Zeit } \mathbf{I}^{2}}$ & $\underline{\text { Zeit II }^{2}}$ & $\frac{\text { Lern- }}{\text { gewinn } I^{2}}$ & $\begin{array}{l}\stackrel{\text { Lern- }}{\text { gewinn } \text { II }^{2}} \\
\text { gen }\end{array}$ \\
\hline & $\underline{\mathbf{r}}_{\mathrm{s}}:$ & $\underline{\mathbf{r}}_{\mathrm{s}}:$ & $\underline{\mathbf{r}}:$ & $\underline{\mathbf{r}}:$ & $\underline{\mathbf{r}}:$ & $\underline{\mathbf{r}}:$ \\
\hline $\begin{array}{l}\text { Hippocampus } \\
\text { rechts }\end{array}$ & -.049 & -.017 & -.073 & .039 & -.052 & .147 \\
\hline $\begin{array}{l}\text { Hippocampus } \\
\text { links }\end{array}$ & .333 & 201 & .252 & .160 & -.233 & -.161 \\
\hline
\end{tabular}

Testverfahren und Prüfgröße: ${ }^{1}$ Spearman Rangkorrelation, $r_{s} ;{ }^{2}$ Pearson-Korrelation, $r$.

\subsubsection{Ergänzung zu 4.2.2.1. (Schizophrene Subgruppen - Navigationsleistung)}

Tabelle 32: $\quad$ Subtypenanalyse, post-hoc-Analysen der Navigationsleistung, Haupteffekt „Subgruppe“

\begin{tabular}{|c|c|c|c|c|c|c|}
\hline \multirow[t]{2}{*}{ Indikator: } & \multicolumn{2}{|c|}{$\begin{array}{l}\text { Paranoid vs. } \\
\text { desorganisiert }\end{array}$} & \multicolumn{2}{|c|}{$\frac{\text { Paranoid vs. }}{\text { gesund }}$} & \multicolumn{2}{|c|}{$\frac{\text { Desorganisiert vs. }}{\text { gesund }}$} \\
\hline & $\underline{\mathbf{F}(\mathbf{d f})}{ }^{\#}:$ & Signifikanz & $\underline{\mathbf{F}(\mathbf{d f})^{\#}:}$ & Signifikanz & $\underline{\mathbf{F}(\mathbf{d f})}{ }^{\#}:$ & $\underline{\text { Signifikanz }}$ \\
\hline Fehlersumme I & $0,240(1)$ & .624 & $11,001(1)$ & $.001 * *$ & 7,634 (1) & $.006 * *$ \\
\hline Fehlersumme II & $0,223(1)$ & .637 & $8,601(1)$ & $.003 * *$ & $6,118(1)$ & $.013 *$ \\
\hline Zeit I & $0,478(1)$ & .489 & $10,229(1)$ & $.001 * *$ & $8,184(1)$ & $.004 * *$ \\
\hline Zeit II & $0,581(1)$ & .446 & 7,777 (1) & $.005 * *$ & $11,434(1)$ & $<.001 * *$ \\
\hline
\end{tabular}

${ }^{\#}$ Testverfahren und Prüfgröße = non-parametrische post-hoc ANOVA, F-Wert; df = Freiheitsgrade; * $=$ signifikant bei $\alpha<0.05, * *=$ signifikant bei $\alpha<0.01$.

Tabelle 33: $\quad$ Subtypenanalyse, post-hoc-Analysen der Navigationsleistung; Haupteffekt „Testumwelt“

\begin{tabular}{|c|c|c|c|c|c|c|}
\hline \multirow[t]{2}{*}{ Indikator: } & \multicolumn{2}{|c|}{$\frac{\text { Paranoid vs. }}{\text { desorganisiert }}$} & \multicolumn{2}{|c|}{$\frac{\text { Paranoid vs. }}{\text { gesund }}$} & \multicolumn{2}{|c|}{$\frac{\text { Desorganisiert vs. }}{\text { gesund }}$} \\
\hline & $\underline{\mathbf{F}(\mathbf{d f})}{ }^{\#}:$ & Signifikanz & $\underline{\mathbf{F}(\mathbf{d f})}{ }^{\#}:$ & Signifikanz & $\underline{\mathbf{F}(\mathbf{d f})}{ }^{\#}:$ & Signifikanz \\
\hline Fehlersumme I & $4,975(1)$ & $.026^{*}$ & $12,787(1)$ & $<.001 * *$ & $6,547(1)$ & $.011^{*}$ \\
\hline Zeit I & $1,340(1)$ & .247 & $8,650(1)$ & $.001 * *$ & $2,413(1)$ & .120 \\
\hline
\end{tabular}

${ }^{\#}$ Testverfahren und Prüfgröße = non-parametrische post-hoc ANOVA, F-Wert;df = Freiheitsgrade; * $=$ signifikant bei $\alpha<0.05, * *=$ signifikant bei $\alpha<0.01$. 
Tabelle 34: $\quad$ Subtypenanalyse, post-hoc-Analysen der Navigationsleistung; Interaktionseffekt „Subgruppe“ - „Testumwelt“

\begin{tabular}{|c|c|c|c|c|c|c|}
\hline \multirow{2}{*}{ Indikator: } & \multicolumn{2}{|c|}{$\begin{array}{c}\text { Paranoid vs. } \\
\text { desorganisiert }\end{array}$} & \multicolumn{2}{|c|}{$\frac{\text { Paranoid vs. }}{\text { gesund }}$} & \multicolumn{2}{|c|}{$\frac{\text { Desorganisiert vs. }}{\text { gesund }}$} \\
\hline & $\underline{\mathbf{F}(\mathbf{d f})}{ }^{\#}:$ & Signifikanz & $\underline{\mathbf{F}(\mathbf{d f})^{\#}}: \underline{\underline{:}}$ & Signifikanz & $\underline{\mathbf{F}(\mathbf{d f})^{\#}} \stackrel{:}{=}$ & $\underline{\text { Signifikanz }}$ \\
\hline Lernkurve I & $5,657(1)$ & $.017 *$ & $0,274(1)$ & .601 & $4,718(1)$ & $.030 *$ \\
\hline
\end{tabular}

${ }^{\#}$ Testverfahren und Prüfgröße = non-parametrische post-hoc ANOVA, F-Wert; df = Freiheitsgrade; * $=$ signifikant bei $\alpha<0.05$. 


\subsection{Ergänzende inhaltliche Informationen}

\subsubsection{Virtuelle Realität}

\subsubsection{Komponenten eines VR-Systems}

1) Computer/Systemsteuerung ${ }^{26}: \quad$ Graphikcomputer und VR-Software. Liefern Daten und Algorithmen für die geometrische und dynamische Beschaffenheit der virtuellen Umgebung.

2) Output devices/Anzeigesysteme: Präsentieren dem Benutzer die relevanten (visuellen, akustischen, taktilen...) Reize der virtuellen Umgebung

Varianten: Head Mounted Displays (HMD; Datenbrillen), ShutterBrillen mit Computerbildschirm, Einwand- und Mehrwandprojektionssysteme (z.B. "CAVE”), DesktopVR, Augmented Reality

3) Input devices/Eingabesysteme: Erfassen Verhaltensweisen des Probanden, die zur Generierung/Adaptation der virtuellen Umwelt wesentlich sind (z.B. Körper-, Kopf-, Hand-, Augenbewegungen, Sprache...); dienen der Interaktion von Testperson und VR

Varianten: Tracking-Systeme, taktil-haptische Systeme, "PrimaryUser-Input-Systeme", “Whole-Body-Motion-Systeme", Mäuse, Joysticks, Spracheingabesysteme

\subsubsection{2. (Weitere) Vorzüge des VR-Paradigmas}

Neben den unter 1.2.2.2.2. dargestellten Vorzügen besitzen Virtuelle Realitäten des Weiteren den Vorzug, dass sie auch physische Räume abbilden können, die in der Realität nicht begehbar sind (z.B. anatomische Strukturen; vgl. Ota et al., 1995; Müller et al., 1998), die zu abstrakt oder zu gefährlich sind (z.B. Feuerwehr- und Militäreinsätze, vgl. Witmer et al., 1996; Bliss et al., 1997) oder deren Aufsuchen nicht möglich ist (z.B. Konfrontation bei Flugangst; vgl. Rothbaum et al., 1996; militärisches Kriegstraining; Vargas, 2006). „VE surrogates for real world places are most useful when it is not possible, practical or safe to use the actual physical location to train. VE surrogates can provide the means to train military units or police officers to rescue hostages, firefighters to extinguish fires in large buildings or astronauts to repair satellites in space" (Witmer et al., 1996, S. 414).

\footnotetext{
${ }^{26}$ Eine genaue Erläuterung der technischen Parameter sowie Vor- und Nachteile der verschiedenen Varianten findet sich bei Mehlitz, 2004.
} 
Im klinischen Setting werden Virtuelle Realitäten insbesondere für Konfrontationsübungen im Rahmen von Angsttherapien eingesetzt (vgl. Tabelle 35; siehe zudem Glantz et al., 1997). Die virtuelle Konfrontation besitzt im Vergleich zu Konfrontationen in-vivo den Vorteil, dass sie von Betroffenen als weniger aversiv und schambesetzt empfunden wird und somit eine höhere Akzeptanz findet. Da eine maximale Stimuluskontrolle besteht und die angstbesetzten Stimuli in ihrer Bedrohlichkeit in sehr feiner Abstufung variiert werden können (z.B. Variation der Enge des Raumes bei Klaustrophobie), erfahren die Patienten ein subjektives Kontroll- und Sicherheitsgefühl, welches die Begegnung mit dem phobischen Reiz erleichtert (Carlin et al., 1997; North et al., 1997b; Botella et al., 1998; Costa et al., 2002). Das Paradigma impliziert aufgrund seiner geringen Verweigerungsrate folglich ein großes Potenzial, um die Inanspruchnahmepopulation von Angsttherapien zu erhöhen, die trotz Erfolgsraten von 75-95 Prozent nur bei 15 Prozent liegt (vgl. GarciaPalacios, 2001 und Garcia-Palacios, 2004).

Insbesondere im Hinblick auf rehabilitative Ziele scheint zudem die hohe intrinsische Motivation wesentlich, die mittels interaktiver Verfahren erreicht werden kann (vgl. Costa et al., 2002). So ist es auch nicht verwunderlich, dass konventionelle, neuropsychologische Testverfahren zunehmend in das VR-Paradigma übertragen werden, wie zum Beispiel der Wisconsin Card Sorting Test (vgl. Ku et al., 2003) oder die dot-probe-task (vgl. Banos et al., 2004).

\subsubsection{Nachteile und Risiken des VR-Paradigmas}

Trotz aller Vorteile sollten kritische Stimmen im Hinblick auf den Einsatz Virtueller Realitäten nicht unbeachtet bleiben: Nach Biocca (1992) besteht ein wesentliches Hindernis für eine breite Anwendung Virtueller Realität in der Entstehung unerwünschter Nebenwirkungen, wie beispielsweise der „simulator sickness“ (vgl. auch Pausch et al., 1992). Die „simulator sickness“ ist vor allem aus der Verwendung von Flugsimulatoren bekannt und umfasst einen Symptomkomplex von Übelkeit, Schwindel, Erbrechen, Kopfschmerzen und Müdigkeit der Augen. Neben ethischen Problemen führt diese Unverträglichkeit zu einer Ablenkung von der eigentlichen Testaufgabe. Zudem ist bekannt, dass eine negative Korrelation zwischen der „simulator sickness“ und dem „,sense of presence" besteht (vgl. Witmer et al., 1996). Kottlow et al. (2004) sind allerdings zuversichtlich, dass Nebenwirkungen des VR-Paradigmas immer seltener auftreten werden, da die Computertechnologien immer ausgefeilter und somit die Ursachen für das 
Auftreten der „simulator sickness“ - wie zeitliche Latenzen oder unausgereifte Ein- und Ausgabesysteme - in Zukunft kaum noch vorhanden sein werden. ${ }^{27}$

Ein weiterer, häufig angeführter Nachteil der VR sind die relativ hohen Geräte- und Entwicklungskosten (vgl. Mehlitz, 2004). Rothbaum et al. (1996) sind allerdings optimistisch, dass sich dies ähnlich wie beim PC schnell ändern wird und ,in-vitroExpositionen" schon sehr bald in der durchschnittlichen psychotherapeutischen Praxis zu finden sein werden.

Riva et al. (2004) stellen die Frage, warum Virtuelle Realitäten trotz einer Vielzahl an Effektivitätsstudien (vgl. 1.2.2.2. und 7.5.1.4.) im Gesundheitssystem bisher nur wenig etabliert sind. Ihrer Meinung nach liegt dies an der mangelnden Standardisierung der verwendeten Technologien und eingesetzten Methoden. Bis heute seien nur vier Manuale zur Behandlung von Flugangst, Panikstörungen, Essstörungen und Sozialer Phobie publiziert. Um diesem Missstand entgegenzuwirken, offerieren sie als erste Arbeitsgemeinschaft ein standardisiertes Protokoll zur virtuell-realen Behandlung der Panikstörung im Internet (vgl. Riva et al., 2004) - in der Hoffnung, eine Verbreitung des Paradigmas in der psychotherapeutischen Praxis zu fördern.

\subsubsection{Einsatzfelder}

Nach Ansicht von Pimentel und Teixera (1995) stellt das Paradigma der Virtuellen Realität eine Schlüsseltechnologie für vielfältige Anwendungen in Technik, Naturwissenschaften und der Unterhaltungsindustrie dar. Tatsächlich setzen sich computergestützte Versuchsdesigns zunehmend durch - und das in so vielfältigen Disziplinen wie Psychologie, Ingenieurswissenschaften, Architektur, Medizin, der medizinischen und militärischen Aus- und Weiterbildung sowie in den humanen Neurowissenschaften (vgl. Whalley et al., 1995; Witmer et al., 1996; Glantz et al., 1997; Carrozzo \& Lacquaniti, 1998; Mehlitz et al., 1998). Tabelle 35 gibt einen Überblick über die verschiedenen Einsatzgebiete des Paradigmas (vgl. auch 7.5.1.5.).

\footnotetext{
${ }^{27}$ Einen Überblick über diskutierte Ätiologien der simulator sickness, den potentiellen Implikationen für die Verbreitung virtueller Technologien sowie eventuelle Gegenmaßnahmen geben Biocca (1992) und Pausch et al. (1992). Eine aufwendige Untersuchungsreihe zu Nebenwirkungen der Virtuellen Realität findet sich bei Cobb et al. (1999).
} 
Tabelle 35: $\quad$ Typische Einsatzfelder von VR-Technologien - mit exemplarischen Anwendungsbeispielen (modifiziert und ergänzt nach Mehlitz et al., 1998)

\begin{tabular}{|c|c|c|}
\hline & iagnostik $^{1}$ & $\begin{array}{l}\text { Dreidimensionale, interaktive Aufbereitung radiologischer Daten zur non-invasiven } \\
\text { Diagnostik, z.B.: } \\
\text { - } \quad \text { virtuelle Endoskopie (Ota et al., 1995; Satava, 1995) } \\
\text { - } \quad \text { virtuelle Koloskopie (Fenlon et al., 1999) }\end{array}$ \\
\hline & Diagnostik $^{2}$ & $\begin{array}{l}\text { Erfassung kognitiver Funktionen bei Patienten mit Hirnläsionen; Prognose der } \\
\text { Alltagstüchtigkeit im Hinblick auf: } \\
\text { - } \\
\text { - }\end{array}$ \\
\hline 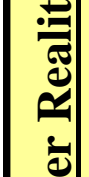 & Therapie $^{1}$ & $\begin{array}{l}\text { Präoperative Planung und intraoperative Navigation mittels 3D-Visualisierung, z.B.: } \\
\text { - } \quad \text { Neuronavigation (Haberland et al., 1997) } \\
\text { - } \quad \text { virtuelle Operation, ,,endoscopic surgery“ (Dumay \& Jense, 1995) }\end{array}$ \\
\hline 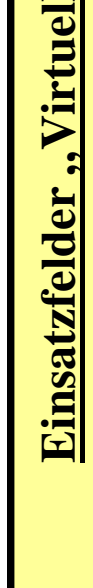 & Therapie $^{2}$ & $\begin{array}{ll}\text { Konfrontationsverfahren bei Angststörungen durch ,in-vitro“-Exposition, z.B. bei: } \\
\text { - } & \text { Acrophobie (Höhenangst; Rothbaum et al., 1995; Huang et al., 2000) } \\
\text { - } & \text { Klaustrophobie (Costa et al., 2002) } \\
\text { - } & \text { Agoraphobie (North et al., 1996) } \\
\text { - } & \text { Flugangst (Rothbaum et al., 1996; North et al., 1997a; Mühlberger et al., 2001, } \\
& \text { 2005; Graap et al., 2004) } \\
\text { - } & \text { Ophidiophobia (Schlangenangst; Parrott et al., 2004) } \\
\text { - } & \text { Arachnophobie (Carlin et al., 1997; St-Jaques et al., 2004) } \\
\text { Selbsthilfe bei HIV-Patienten (Hamza et al., 2004) } \\
\text { Prävention und Intervention bei Essstörungen (Riva, 1997; Winzelberg et al., 2004) }\end{array}$ \\
\hline & $\frac{\text { Rehabili- }}{\text { tation }^{2}}$ & $\begin{array}{l}\text { Rehabilitation von motorischen und kognitiven Beeinträchtigungen, z.B. bei: } \\
\text { - } \quad \text { psychiatrischen Störungen wie Schizophrenie, Autismus, ADHD (Costa et al., } \\
\text { 2002) } \\
\text { - Patienten mit Hirnschädigungen (Pugnetti et al., 1995; Carozzo \& Lacquaniti, } \\
\text { 1998) } \\
\text { Testbatterie zur Evaluation von VR für das Training kognitiver Funktion (Lampton } \\
\text { et al., 1994; Kottlow et al., 2004) }\end{array}$ \\
\hline
\end{tabular}

1 = Behandelnder interagiert mit der VR (mit Patientendaten oder Modellen); 2 = Patient interagiert mit der VR.

\footnotetext{
${ }^{28}$ Ein umfassender Überblick über die verschiedenen Anwendungsfelder der VR findet sich in „Abstracts from Cyber Therapy 2004“ in CyberPsychology and Behavior, 269-320.
} 
Tabelle 35: $\quad$ Typische Einsatzfelder von VR-Technologien - mit exemplarischen Anwendungsbeispielen (Fortsetzung)

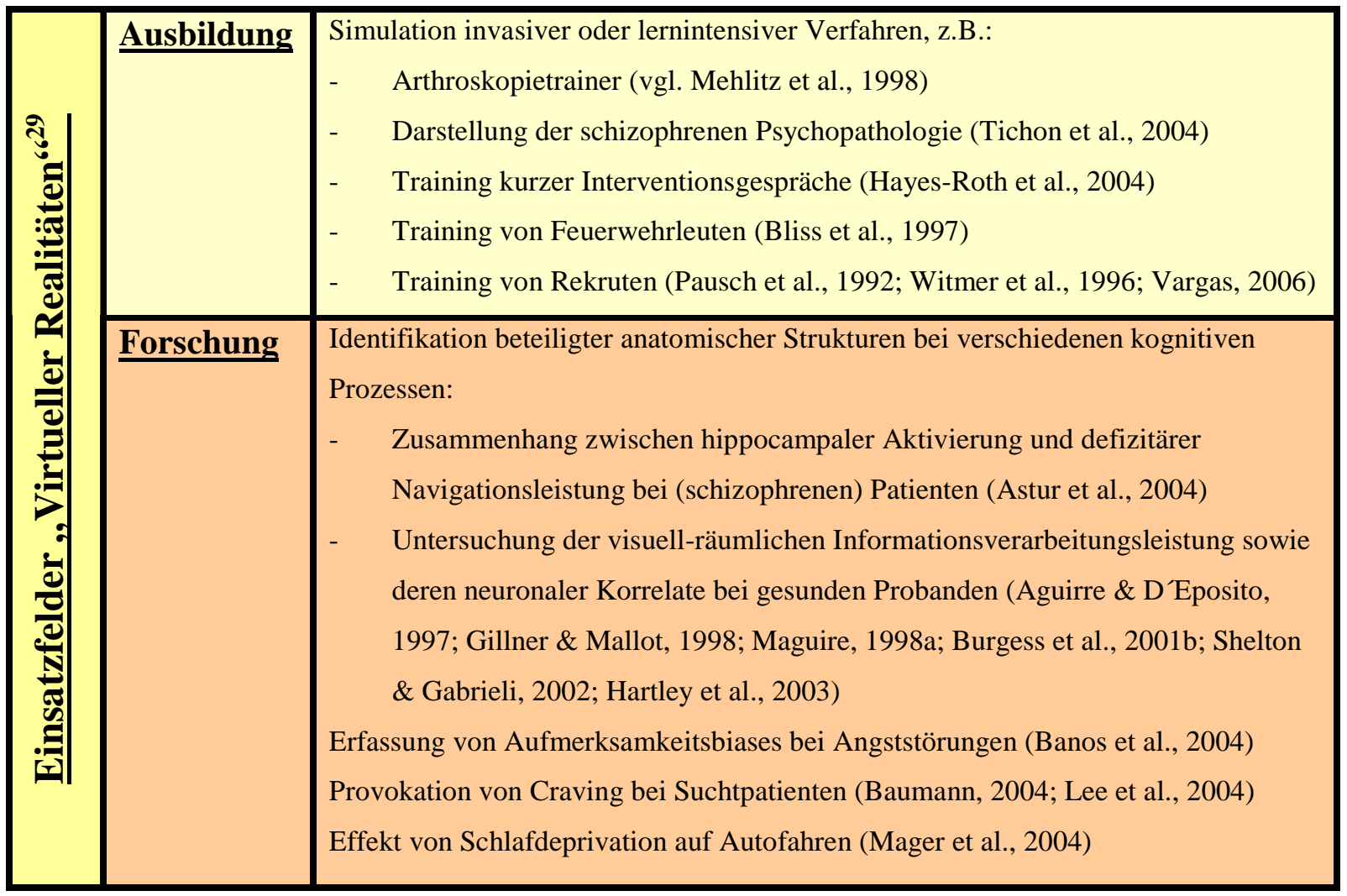

Wie aus der Abbildung ersichtlich, gibt es zwei unterschiedliche Formen der VR: Bei der patientenbasierten Version interagiert der Patient zu diagnostischen, therapeutischen oder rehabilitativen Zwecken mit dem Computersystem. Bei der mediziner- (oder allgemeiner: anwender-) basierten Version interagiert hingegen der Experimentator mit der virtuellen Umgebung (z.B. in dem er mit Hilfe von Patientendaten anatomische Strukturen simuliert).

Insbesondere psychotherapeutische, psychiatrische und neurologische Disziplinen erkennen zunehmend die Vorzüge des VR-Paradigmas (für einen Überblick siehe Glantz et al., 1997). Evaluationsstudien konnten bereits eine deutliche Wirksamkeit des VRParadigmas im Hinblick auf Arachnophobie, Agoraphobie, Acrophobie (Höhenangst), Flugphobie, Ophidiophobie (Schlangenangst) und Klaustrophobie nachweisen, wobei sich die Angstreduktionen im Rahmen von follow-up Erhebungen als stabil erwiesen (vgl. North et al., 1996; Mühlberger et al., 2001; Graap et al., 2004). Zudem gibt es Hinweise darauf, dass die Konfrontation im realen Setting durch eine vorherige virtuelle Konfrontation erleichtert wird (Rothbaum et al., 1996; Carlin et al., 1997).

\footnotetext{
${ }^{29}$ Ein umfassender Überblick über die verschiedenen Anwendungsfelder der VR findet sich in „Abstracts from Cyber Therapy 2004“" in CyberPsychology and Behavior, 269-320.
} 
Im medizinischen Bereich dient das Paradigma insbesondere der dreidimensionalen Rekonstruktion von Patientendaten auf der Basis zweidimensionaler bildgebender Verfahren (wie MRT oder CT) zur Diagnostik, Operationsplanung und robotergestützten Operationsdurchführung (vgl. Tabelle 35 und Mehlitz et al., 1998). Komplexe Eingriffe können mit Hilfe dieser neuen Technologie zunächst risikofrei trainiert werden, bevor sie an Patienten durchgeführt werden. Mit Hilfe der Virtuellen Realität können Fähigkeiten und noch offener Trainingsbedarf junger Ärzte objektiv eingeschätzt werden (vgl. Ota et al., 1995; Satava, 1995). Zudem sind computergenerierte Programme jederzeit verfügbar und erlauben die Einsparung von Tiermodellen, deren Einsatz ohnehin einer starken Kritik ausgesetzt ist (vgl. Mehlitz et al., 1998).

\subsubsection{Interessante Links im Internet}

- Umfassender Überblick über VR-Technologien und Links zu aktuellem Forschungsstand und praktischen Anwendungen: http://de.wikipedia.org/wiki/Virtuelle_Realit\%C3\%A4t

- VR-Expositionstherapie spezifischer Phobien: Flugangst, Angst vor Spinnen, Höhenangst, Tunnelangst: http://www.psychologie.uni-wuerzburg.de/psy1/cs/phobien.html

- Abteilung Virtuelle und Erweiterte Realität des Fraunhofer IGD: http://www.igd.fhg.de/igd-a4/

- Competence Center Virtual Environments des Fraunhofer IAO: http://ve.iao.fhg.de/portal.de.php

- Expositionstherapie mit VR: http://www.psycho-vision.de/index.asp; vor allem: http://www.psychovision.de/vrexpo.asp?article $\mathrm{id}=12$

- $\quad$ Klinischer Einsatz von VR-Technologien für Diagnostik, Therapie und Rehabilitation in der Neurologie und in der Psychiatrie: http://www.ehto.be/hat_projects/vrepar/index.html

- Einsatz dreidimensionaler High-Tech-Software für Teleoperationen, OP-Planung und OPSimulation: http//iregt1.ia.fzk.de/KISMET/kismet.html

- Virtual Dimension Center (VDC), Kompetenzzentrum für Virtuelle Realität: http://www.vdcfellbach.de/

- Virtual Development and Training Center: http://www.vdtc.de/

- Eine freie Implementierung einer VR-Umgebung in Java: http://www.3dchat.org/

- Virtual Reality Center Production Engineering (VRCP) der TU Chemnitz: http://www.vrcp.de/

- Virtual Engineering Software: http://www.icido.de/de/index 1024

- VRVis Zentrum für Virtual Reality und Visualisierung: http://www.vrvis.at/home de.html

- Innovation Center Virtual Reality: http://www.icvr.ethz.ch/

- Human Interface Technology Lab, Seattle, USA: VR Projekte in den Bereichen Chirurgie, Psychologie, Architektur u.ä.; insbesondere VR-Behandlung von Spinnenphobien: http://www.hitl.washington.edu/projects/index.html.

- Cybernarium Darmstadt: http://cybernarium.de/content/home

- Surgical Research; Einführung moderner Computertechnologie (u.a. VR) in neue OP-Konzepte; v.a. für die operative Tumortherapie: http://jota.rkk-berlin.de/op2000/ 


\subsubsection{Klassische neuropsychologische Testverfahren: Subtests}

\section{Detaillierte Beschreibung der HAWIE-R Subtests}

Der Untertest Allgemeines Wissen setzt sich aus 24 Fragen zu unterschiedlichen Wissensgebieten zusammen, die in aufsteigender Schwierigkeit präsentiert werden (z.B. „Was ist eine Ode?"). Ziel ist es, bildungs- und kulturunabhängiges Allgemeinwissen der Probanden zu erfragen oder entsprechend Tewes (1991): die „Aufgeschlossenheit der Versuchsperson gegenüber der Umwelt", das heißt das Ausmaß, in dem die Person an externer Information interessiert ist und inwiefern sie fähig ist, diese zu memorieren.

Der zweite verbale Subtest Gemeinsamkeiten finden dient nach Wechsler (1987) der Prüfung des allgemeinen sprachlichen Abstraktionsvermögens sowie der verbal-logischen Konzeptbildungsfähigkeit. Ziel dieser Aufgabe ist es, die Gemeinsamkeit zwischen zwei Begriffen $\mathrm{zu}$ erkennen beziehungsweise den passenden Oberbegriff zwischen 16 Wortpaaren zu finden (Beispiel: Norden-Westen).

Bei dem Handlungstest Bilder ergänzen werden der Versuchsperson Abbildungen von 17 unvollständigen Bildern präsentiert, bei denen das jeweils fehlende Detail (z.B. die Nase in einem Gesicht) entdeckt und benannt werden soll. Dieser Test erfasst die Fähigkeit zum Erkennen wesentlicher visueller Details und setzt die Fähigkeit voraus, zwischen relevanten und irrelevanten Merkmalen unterscheiden zu können.

Das zweite Verfahren zur Schätzung des Handlungs-IQs ist der Mosaiktest. Aufgabe des Probanden ist es, mit Hilfe von farbigen Würfeln möglichst schnell verschiedene Muster $\mathrm{zu}$ reproduzieren, die in zweidimensionalen Abbildungen vorgegeben werden. Die insgesamt neun Muster, die in aufsteigendem Schwierigkeitsgrad unter Vorgabe eines Zeitlimits präsentiert werden, erfassen die visuo-konstruktiven Fähigkeiten der Testperson sowie die Fähigkeit zur Formwahrnehmung und die Fähigkeit, Figuren in ihre Komponenten zu zerlegen. Darüber hinaus erfordert der Mosaiktest mit der mentalen Rotation der verschiedenen Würfel eine weitere Komponente komplexer visuellräumlicher Informationsverarbeitung. 


\section{Detaillierte Beschreibung der WMS-R Subtests}

Der Test Digit Span Forward erfasst die auditive Gedächtnisspanne. Hierfür müssen die Probanden eine gehörte Zahlenreihe unmittelbar seriell wiedergeben. Der Subtest Digit Span Backward erfordert hingegen die Wiedergabe der Zahlenreihe in mental transformierter Form, da die gehörte Zahlenfolge rückwärts reproduziert werden soll. Diese Aufgabe stellt hohe Anforderungen an das Arbeitsgedächtnis sowie an die Aufmerksamkeitsressourcen der Probanden und erfasst die Leistungsfähigkeit des verbalen Kurzzeitgedächtnisses.

Analog zur auditiven Version erfassen die Tests Visual Memory Span Forward und Visual Memory Span Backward die Kurzzeitgedächtnisleistung in der visuell-räumlichen Modalität. Aufgabe des Probanden ist es hier, Bewegungsfolgen des Versuchsleiters auf einem mit Klötzchen beklebtem Brett seriell sowie in umgekehrter Reihenfolge zu replizieren.

Die Subtests Logical Memory I und Logical Memory II erfassen verbale Kurzzeit- und Langzeitgedächtnisfunktionen mit Hilfe von komplexem, verbalem Material. Die Testpersonen sollen kurze Geschichten sofort (verbales Kurzzeitgedächtnis) und nach einer 30-minütigen Latenz (verbales Langzeitgedächtnis) wiedergeben.

Als non-verbales Analogon zur Erfassung visueller Kurz- und Langszeitgedächtnisfunktionen fungieren die Tests Visual Reproduction I und Visual Reproduction II. Die Probanden werden gebeten, vier geometrische Abbildungen unmittelbar und nach einer 30minütigen Pause zu erinnern. 


\section{Wissenschaftlicher Bildungsgang der Verfasserin}

\section{Angaben zur Person}

Name:

Mirjana Ruhleder

Geboren:

07.08.1979 in Bad Harzburg

Familienstand: ledig

Nationalität:

deutsch

1985-1989

\section{Schulbildung}

1989-1991

Besuch der Gerhardt-Hauptmann-Grundschule in Bad Harzburg Besuch der Orientierungsstufe in Bad Harzburg

1991-1998

Besuch des Niedersächsischen Internatsgymnasiums in Bad Harzburg

Abschluss: Allgemeine Hochschulreife

\section{Hochschulausbildung}

1998-2004

Psychologie-Studium an der Julius-Maximilians-Universität in Würzburg

Abschluss: Diplom

2000-2003

Wissenschaftliche Hilfskraft am Lehrstuhl für Klinische und Biologische Psychologie der Universität Würzburg

2003-2004 Verhaltenstherapeutische Zusatzausbildung bei der Arbeitsgemeinschaft für Verhaltensmodifikation (AVM) in Würzburg

Seit 03.2005 Promotion am Universitätsklinikum Göttingen in der Schwerpunktprofessur für Psychopathologie und Neuropsychologie

Seit 04.2005 Weiterführendes Studium zur Psychologischen Psychotherapeutin an der Georg-August-Universität Göttingen (WSPP) 